Ergün Lafl1,

Dokuz Eylül University,

Department of Archaeology, Izmir, Turkey

elafli@yahoo.ca

Gülseren Kan Şahin,

Dokuz Eylül University,

Department of Archaeology, Izmir, Turkey

gulserenkan@hotmail.com
UDK: 903:738"638"(560) ;

904:738"652"(560);

902.2(560)"2005/2008"

ID: 195899916

Original research article

Received: August 07, 2011

Accepted: September 05, 2011

To the Serbo-Turkish friendship

and cooperation in archaeology.

\title{
IRON AGE AND HELLENISTIC CERAMICS FROM SOUTHWESTERN PAPHLAGONIA
}

\begin{abstract}
Paphlagonia was an ancient region on the Black Sea coast of north central Anatolia, bordered by Bithynia to the west, Pontus to the east and Galatia to the south. Between 2005 and 2008 an archaeological team from the Dokuz Eylül University in Izmir carried out archaeological field surveys and excavations in the southwestern part of Paphlagonia. In this extensive report pottery finds from the Iron Age and Hellenistic periods discovered during the fieldwork will be presented in detail. It includes a typological list of pottery sherds that were collected mostly from the surface. For the periods concerned there is more information from Kimistene, a hilltop site in southwestern Paphlagonia, than from other sites. This study is the first detailed Hellenistic pottery report of Turkish Black Sea area.
\end{abstract}

\section{KEY WORDS: PAPHLAGONIA, KIMISTENE, HADRIANOPOLIS, BLACK SEA, ASIA MINOR, TURKEY, IRON AGE, PHRYGIAN, HELLENISTIC.}

Paphlagonia was an ancient region on the Black Sea coast of north central Anatolia, bordered by Bithynia to the west, Pontus to the east, and Galatia to the south (map 1). The archaeology of Paphlagonia has been studied in a very uneven manner: the south coast of the Black Sea has hardly been touched by comparison with the century and more of sustained excavations and surveys on the other coastlines. Inevitably, the archaeological picture of these coasts in antiquity looks strangely unbalanced, even though our literary texts offer moments of insight into the Classical Antiquity of the Turkish Black Sea coast, spread across centuries and driven by Xenophon's Anabasis, Strabo's
Geography and Arrian's Periplus. Centuries later, the whole Turkish coast of the Black Sea is a live archaeological region, and the ongoing discoveries help shed more light on the facts of the past and on the incredible prosperity of this region in antiquity.

Between 2005 and 2008 an archaeological team from the Dokuz Eylül University in Izmir carried out archaeological field surveys (map 2) and excavations in the southwestern part of Paphlagonia, focusing on the site of Hadrianopolis (map 3) near the town of Eskipazar in the Turkish Province of Karabük and on its surrounding area, on its so-called chora (Lafl1/Christof 2012a, 28- 


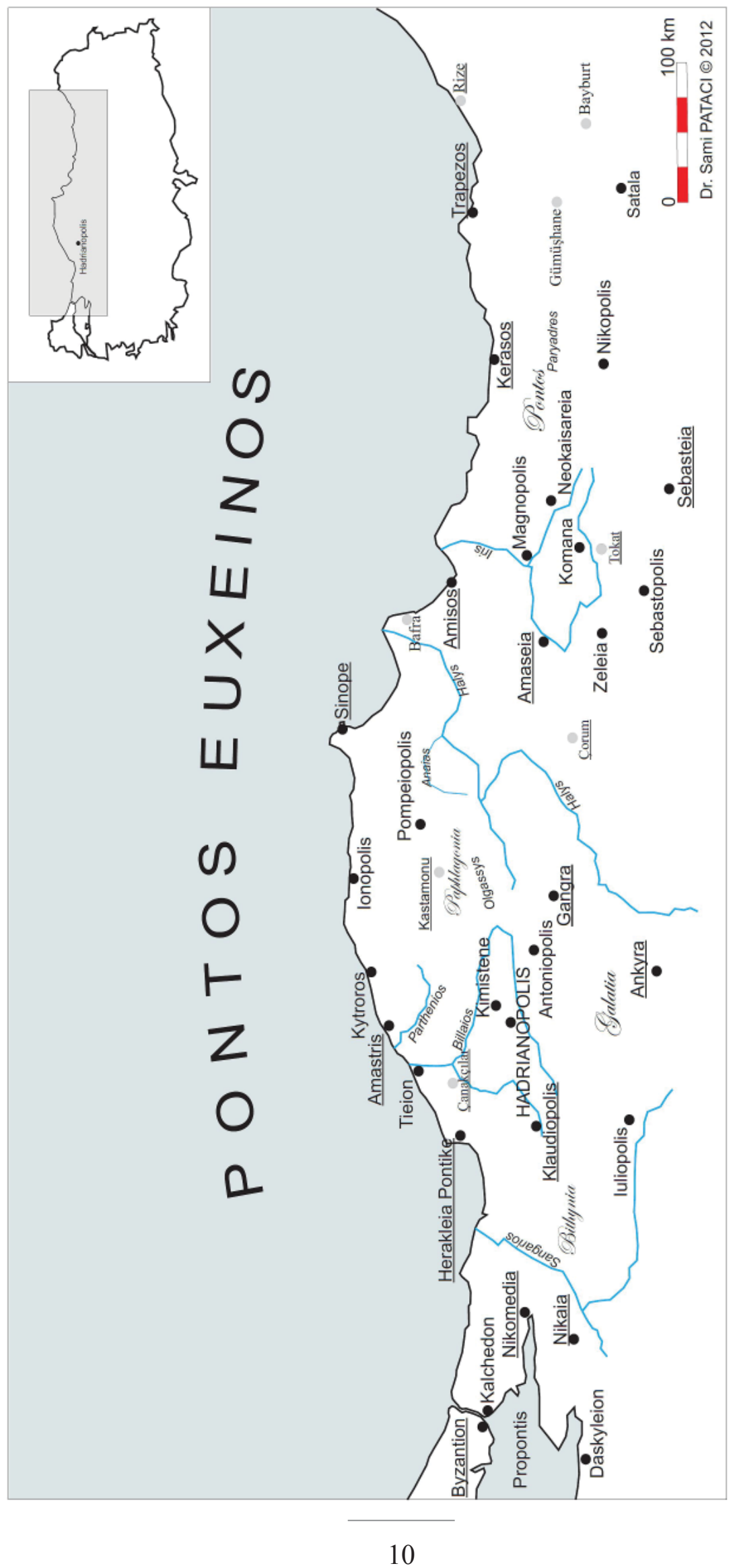

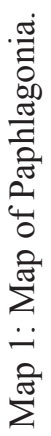




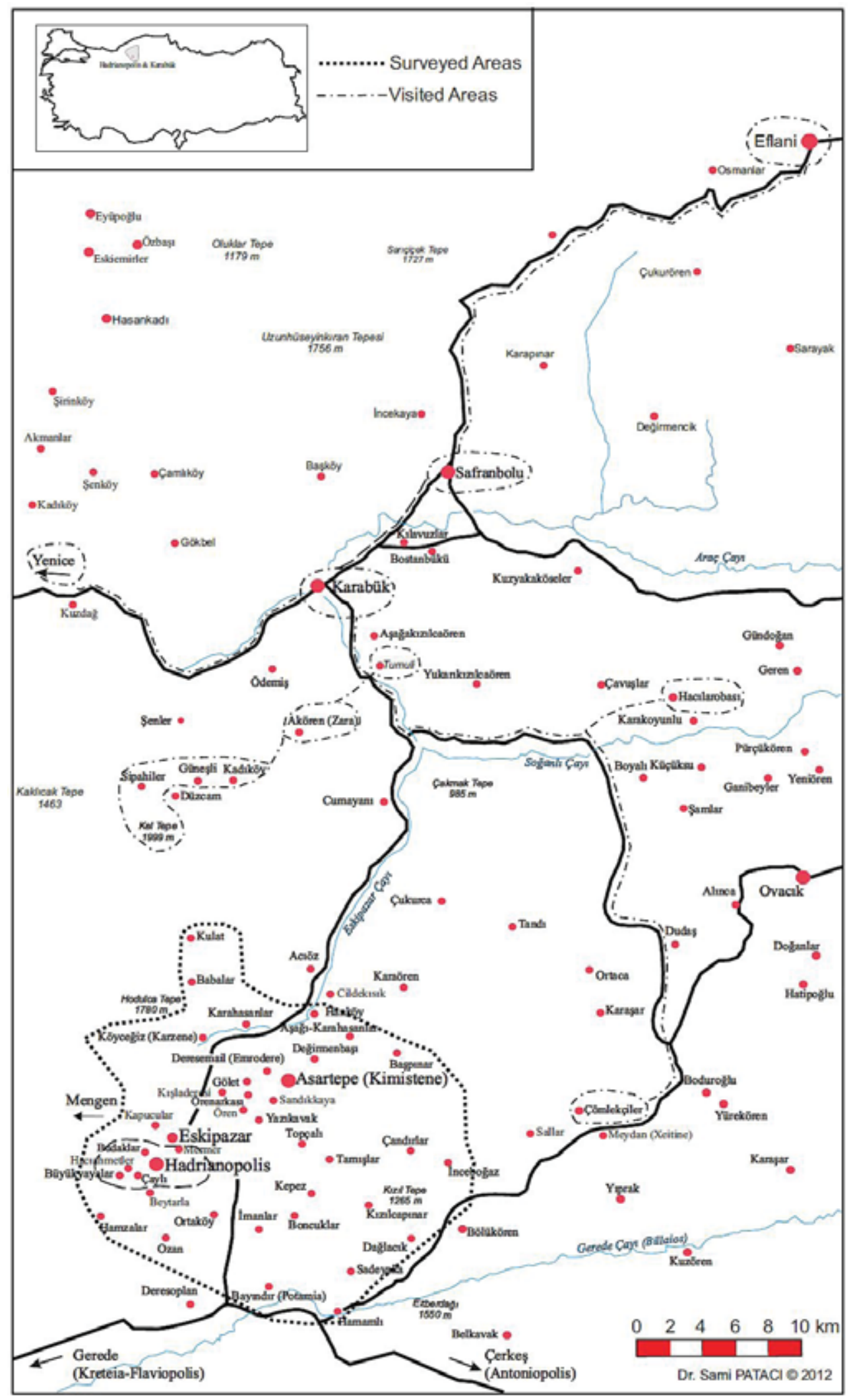

Map 2: Map of the surveyed areas in southwestern Paphlagonia in 2005. 


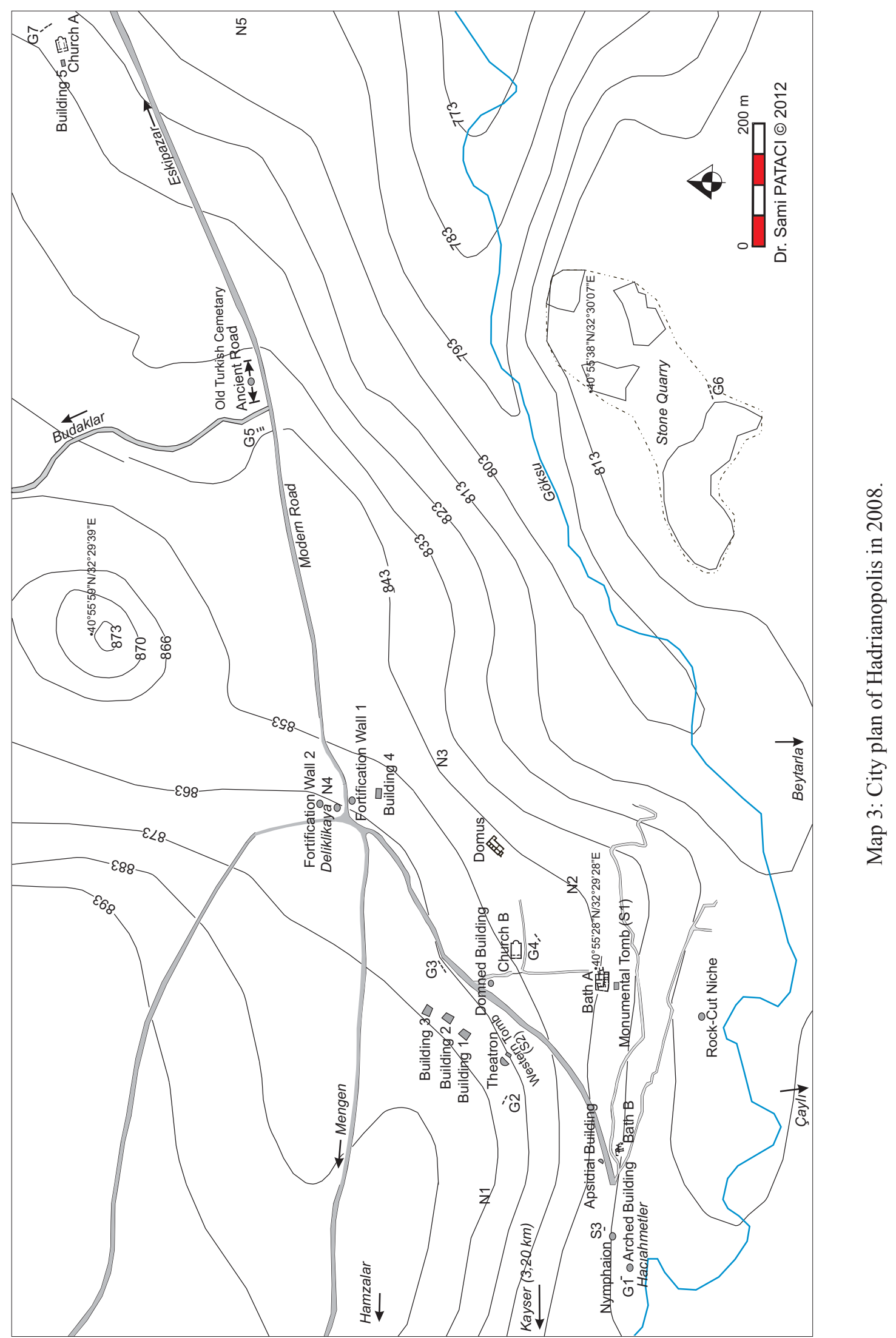


Laflı, Kan Şahin - Iron Age and Hellenistic Ceramics...(9-166) Archaeology and Science 7 (2011)

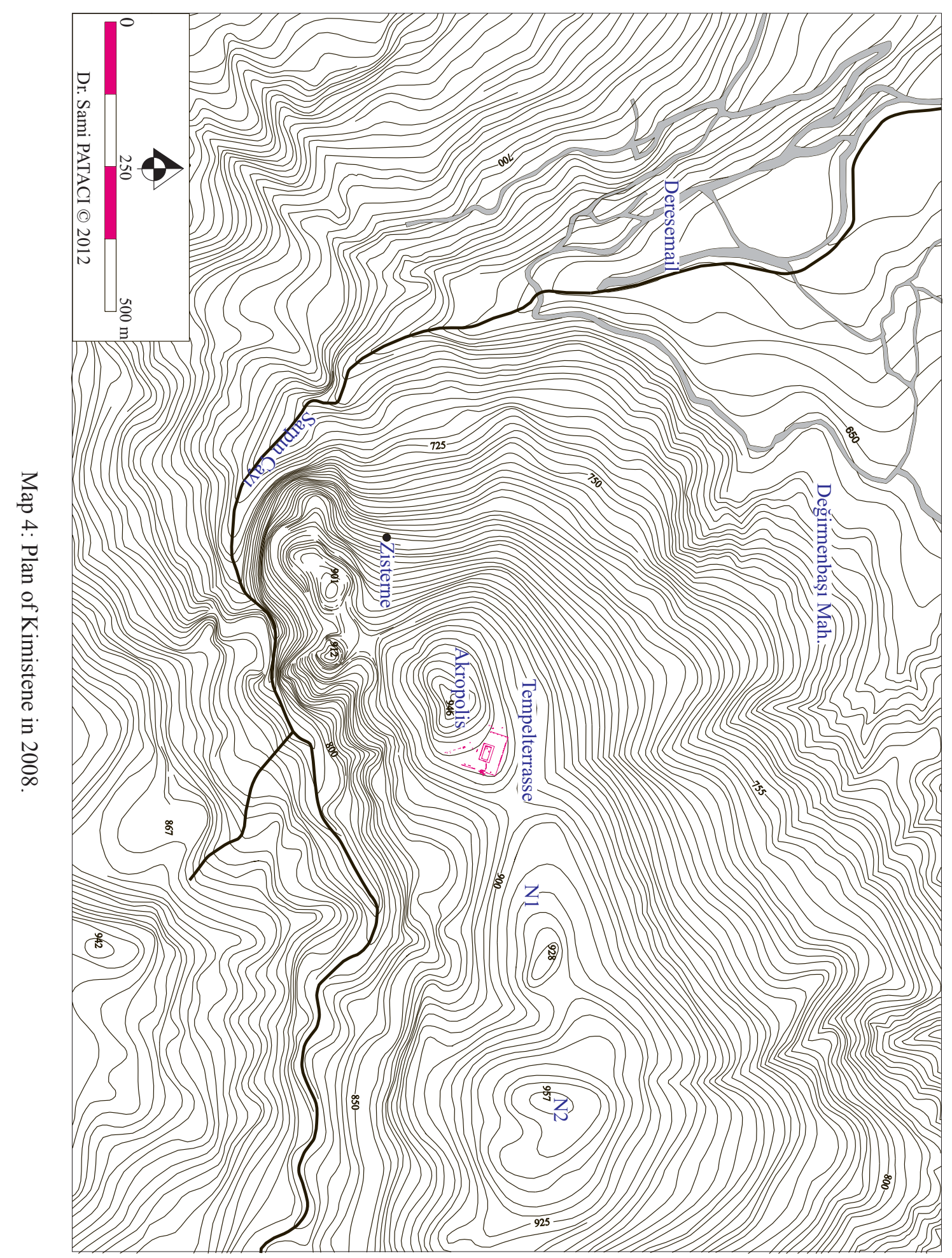




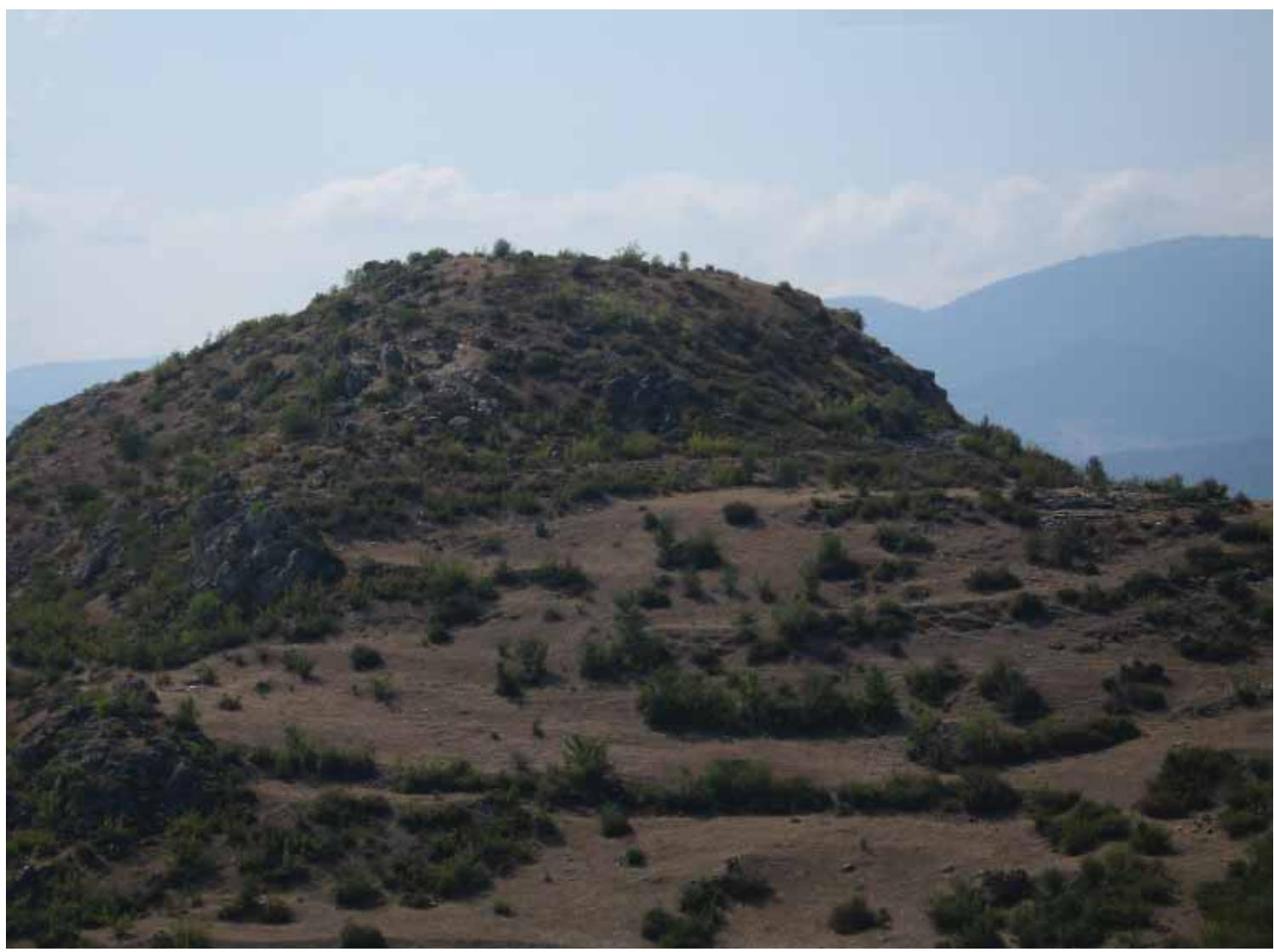

Fig. 1: Acropolis of Kimistene from the north.

31 and Fünfschilling/Laflı 2012, 5). During these field campaigns it has been shown that southwestern Paphlagonia was a transitional landscape between Paphlagonia, Galatia and Bityhnia that was settled without interruption from the Bronze Age until the 8th cent. A.D. The earliest pottery from southwestern Paphlagonia originates from the chora of Hadrianopolis and not from the site of Hadrianopolis itself. In this report pottery finds from the Iron Age and Hellenistic period discovered during the fieldwork will be presented in detail (for a preliminary Iron Age and Hellenistic pottery report of southwestern Paphlagonia: Lafl1/ Kan Şahin in press).

Almost no historical source exists for understanding the situation of southwestern Paphlagonia during the Iron Age. Regarding the Iron Age of the hinterland of Paphlagonia, so-called Inner Paphlagonia, the only systematic archaeological field work carried out to date was led by R.
J. Matthews of the British Institute of Archaeology at Ankara between 1997 and 2001. The Hittite world in central and northern Anatolia collapsed in 1180 B.C. and after the Hittites, the first known people in this region are the Phrygians, whose capital at Gordion is not far to the south-west of Paphlagonia. According to Matthews's survey the Middle Iron Age has been dated to 950-550 B.C. and the Late Iron Age to 550-330 B.C. The British survey showed that the only Iron Age site in the area of Eskipazar was İnceboğaz Tepesi which is indicated as a fortified site (Matthews 2009, 151, table 5.2; map of distributions of Iron Age sites in Inner Paphlagonia: Matthews 2009, 150, fig. 5.1.). In our archaeological field surveys in 2005 we examined Kimistene (map 4), a hilltop site $c$. $12 \mathrm{~km}$ northeast of Hadrianopolis, near the village of Deresemail, and collected Iron Age sherds there which will be presented below. Kimistene is located on a mountain chain, called Asartepe 


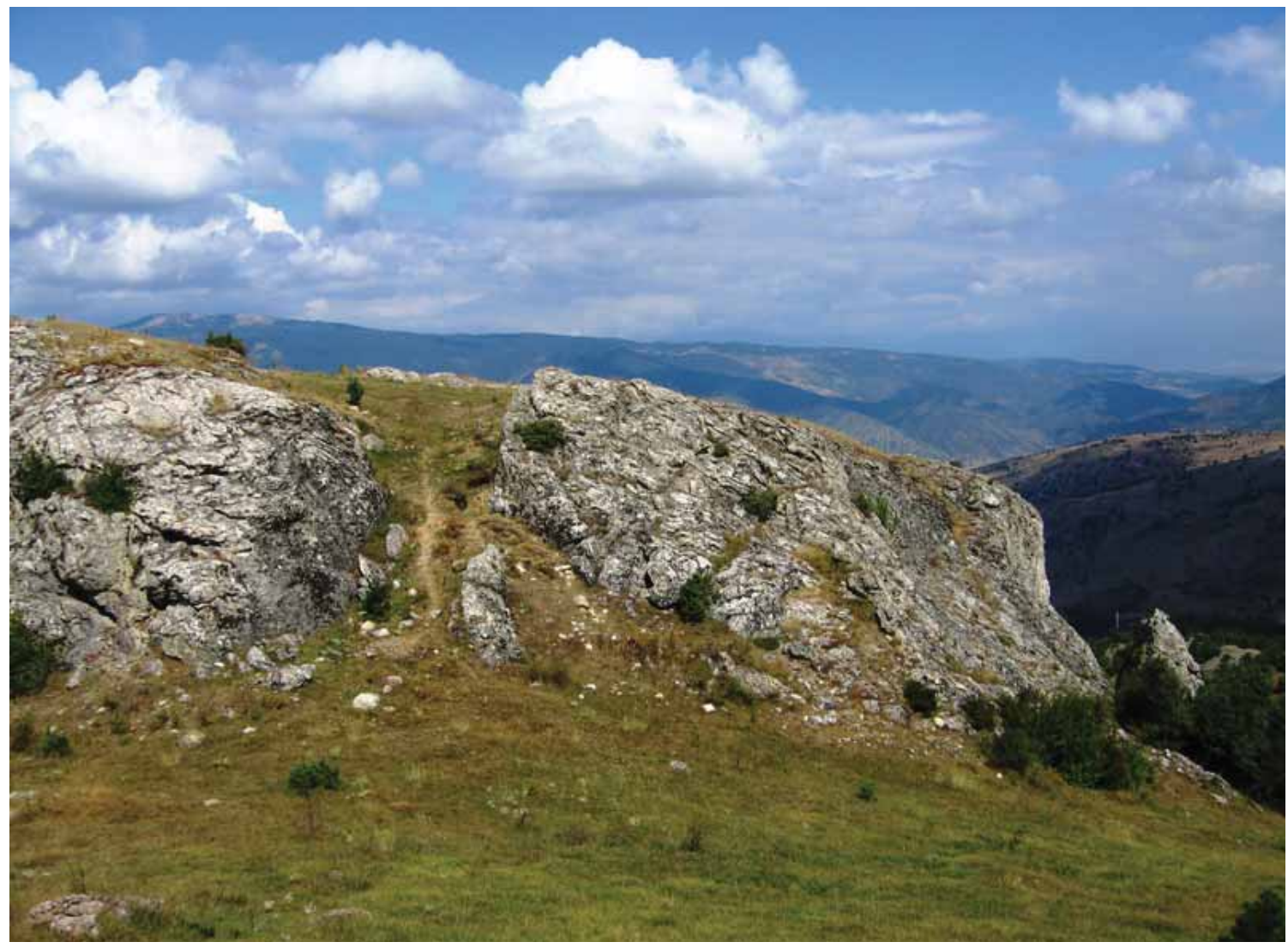

Fig. 2: Kepez from the south.

by the locals, and it consists of four main peaks: Acropolis (fig. 1), cistern, and two necropoleis. According to Matthews, many of the hilltop sites may have been constructed in the Late Iron Age (Hellenistic) period and re-used in the Late Byzantine period.

At the end of 4th century B.C. Paphlagonia passed under the control of the Macedonian kings, and after the death of Alexander the Great it was assigned, together with Cappadocia and Mysia, to Eumenes. However, it continued to be governed by native princes until it was absorbed by the encroaching power of Pontus. The rulers of that dynasty became masters of the greater part of Paphlagonia as early as the reign of Mithridates Ctistes (302-266 B.C.), but it was not until 183 B.C. that Pharnaces brought the Greek city of Sinope under their control. From that time, the whole province was incorporated into the kingdom of Pontus until the fall of Mithridates in 65
B.C. Hellenistic immigrants to the region included the Celtic Galatians who flooded into Anatolia in the 3rd century B.C. In the surveys carried out by Matthews's team there is at least one hill-top fortified site in the south of the survey area that is probably Galatian in date.

The Hellenistic economy of the coastline of Paphlagonia and Pontus was based on agricultural and industrial activities, and especially on wine and oil production and their international trade. Parallel to the intensive wine and oil production, technologically proficient transport amphorae were produced beginning at the latest in the Late Classical period and continuing into the Middle Byzantine period. Sinope, Heracleia Pontica and Amastris, all in Paphlagonian territories, thus became famous during Hellenistic and Roman times for their wine production and trade.

According to D. Magie, however, Hellenistic Inner Paphlagonia had a rural character with 


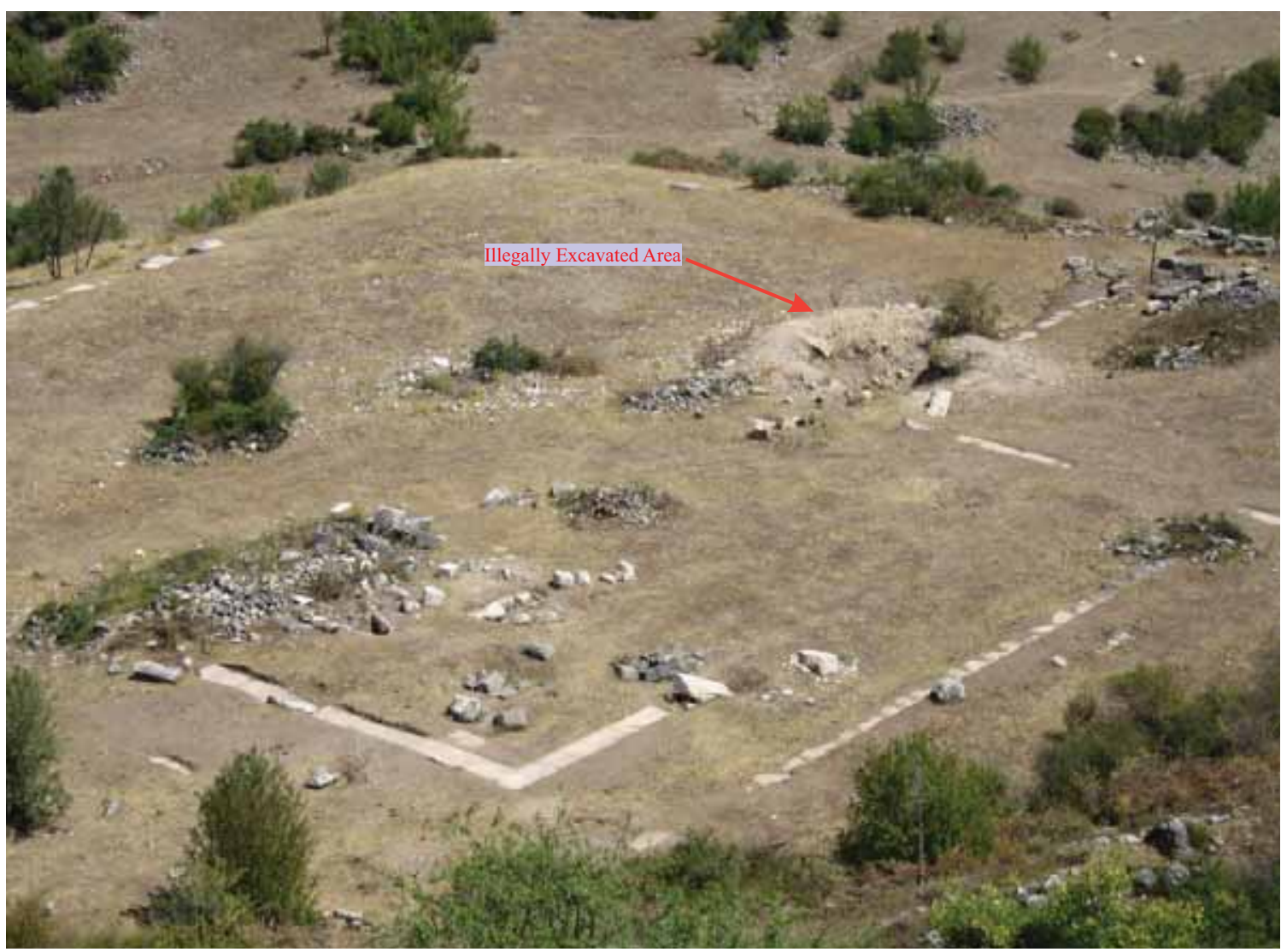

Fig. 3: Illegally excavated area at the temple's podium on the Acropolis of Kimistene in 2005.

a rural type of living (Magie 1950, 188). Almost no Hellenistic cities are known in southwestern Paphlagonia. At the same time one should stress the fact that Inner Paphlagonia (southwestern Paphlagonia) was never a heavily urbanized area (cf. map of Hellenistic Inner Paphlagonia: Matthews/Metcalfe/Cottica 2009, 178, fig. 6.6), and Hellenistic sites are also very rare. The only Hellenistic site in the area that was discovered during the course of Matthews's surveys is PS 066, which is a cemetery site. This is perhaps due to the abandonment of rural settlements (Matthews/Metcalfe/Cottica 2009, 177). The sole evidence for Hellenistic settlement in the region is the tumuli, the date of which is rather problematic (Lafl1/Christof $2012 \mathrm{~b}$; as well as tumuli in the region: Matthews 2009, 157-158, table 5.4 and 159, fig. 5.8). Other parts of Paphlagonia, especially "Outer" Paphlagonia are better represented during the Hellenistic period, but their ceramic evidence has not been published to any great extent. A further problem is that we cannot be certain if southwestern Paphlagonia was incorporated with the wider Hellenistic world by a network of roads.

In our 2005 survey in southwestern Paphlagonia we collected Hellenistic sherds from two major sites: Kimistene and Kepez (fig. 2). The function of Kimistene in the Hellenistic period is unclear (for Hellenistic Kimistene: Matthews/ Metcalfe/Cottica 2009, 174-177; for Kimistene: Lafl1/Christof 2011). At this site traces of foundations of a Roman temple (of Zeus Kimistenos?), located on the northern artificial edge of its Acropolis and on the southwestern flank of the site should have been built during the 3rd century A.D. and should have been in use until the 4th cent. A.D. This temple is an indication of some unknown religious activities at a hilltop sanctuary (cf. hilltop sanctuaries in Paphlagonia: Matthews/ Metcalfe/Cottica 2009, 174-177.). As understood 


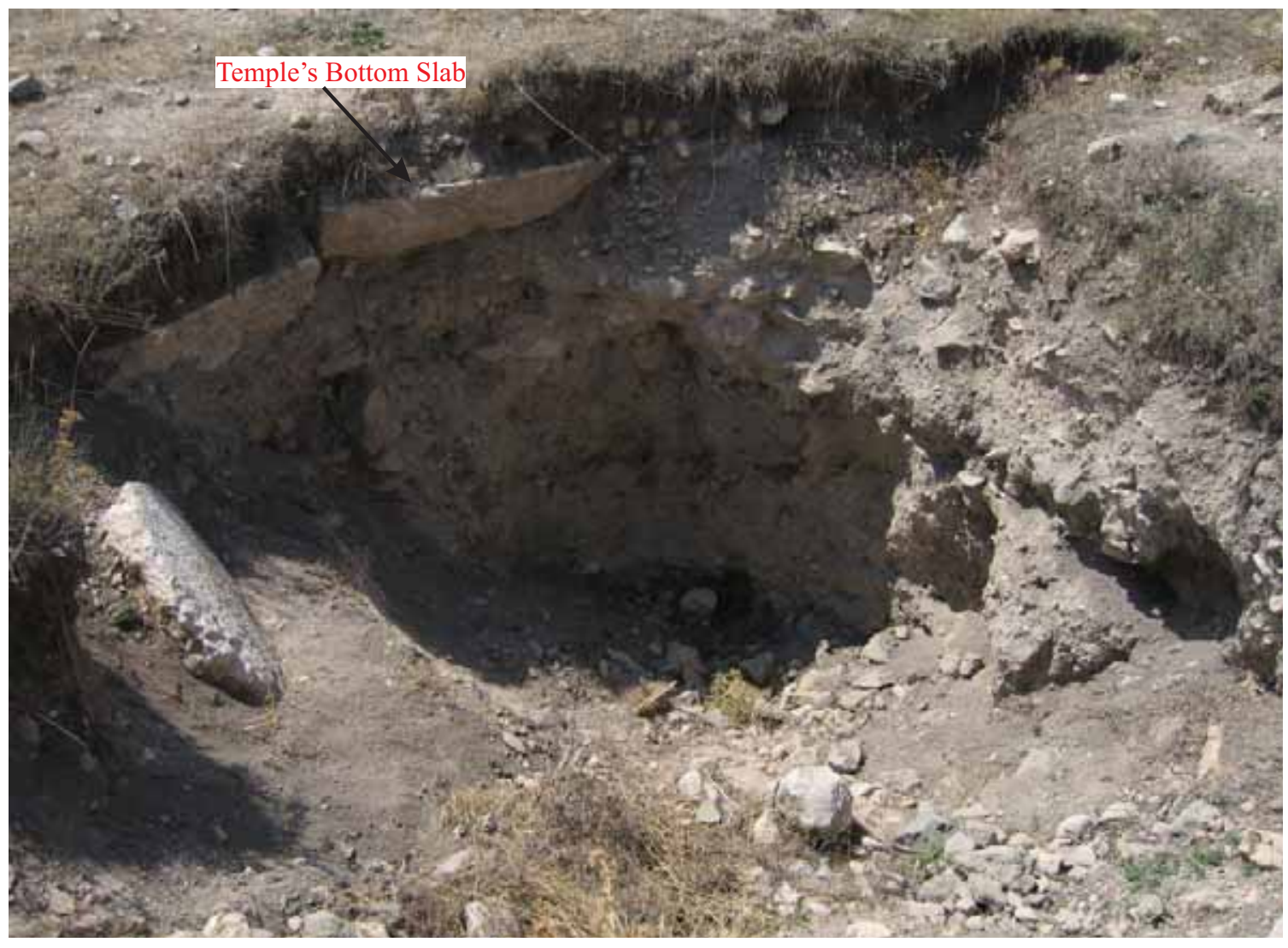

Fig. 4: Illegally excavated area at the temple's terrace in 2005.

from the illegal trenches (figs. 3-4) opened up in its temenos, the foundations of this temple lay in the Iron Age/Hellenistic fill where we have found numerous sherds of Iron Age grey ware, especially open forms, but no painted ware or Hellenistic and Roman pottery. In the illegal excavation trench we documented at least three settlement layers of $2 \mathrm{~m}$ in height, going deeper than the foundation level of the Roman temple. This is perhaps an indication that this area was a cultic area much earlier than the arrival of the Romans. According to some scholars, Kimistene was a base for the Pontic operations of Mithridates Ctistes as he founded and expanded his kingdom in the years around 300 B.C. (Matthews/Metcalfe/Cottica 2009, 177.). During our survey we were not able to discover any architectural indication for such a Hellenistic fortification. The existing fortification on the Acropolis of Kimistene is dateable to the Middle Byzantine period. Kepez is a cemetery site with two rock-cut graves and two cisterns. It is $c .8 \mathrm{~km}$ southeast of Kimistene and it also located in a cliffy area.

Very few pottery earlier than the 1 st cent. B.C. was found in Hadrianopolis, with the exception of a few prehistoric sherds. The first historical sign of this city is that the Caesereia Hadrianopolis region appears to have been annexed to Bithynia already in 63 B.C. (Mitchell 1993, 9293) The archaeological evidence currently suggests that Hadrianopolis was a kata komas polis in the 1st cent. B.C., and it would thus not have had a highly developed urban centre. It was probably only founded in the second quarter of the 1st century B.C. We do not know about the situation at Gangra in the Late Hellenistic period either.

The termination of the 1st century B.C. in the course of Paphlagonian history has a problematic nature as the Romans succeeded in incorporating the region fully into their empire by the end 


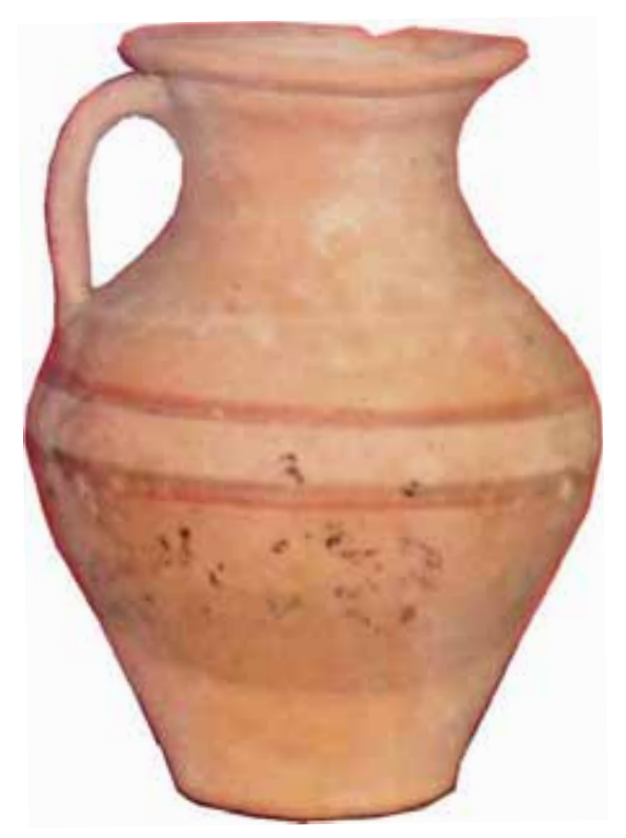

\section{$011233456788910 \mathrm{~cm}$}

Fig. 5: A Hellenistic painted jug from the Museum of Çankırı.

of the first century B.C. (Matthews/Metcalfe/Cottica 2009, 174). It is, therefore, not easy to classify this period either as "Late Hellenistic" or "Early Roman".

Few Hellenistic ceramics have been published from Paphlagonia (Laflı 2006); on the coastline pottery studies are almost exclusively devoted to amphorae. Excavations in the region, such as at Pompeiopolis in eastern Paphlagonia, and Sinope and Tieion on the coast have so far provided little Hellenistic ceramic evidence. Very few sherds were published in the survey reports of the hinterland region.

During the four seasons of field research carried out between 2005 and 2008 in southwestern Paphlagonia a wide range of ceramics was collected from field surveys and excavations; a total of c. 1525 fragments was examined. From these,
36 are classified as "Pre-Iron Age", 92 as "Iron Age" (30 of which are Iron Age coarse ware) and 458 as "Hellenistic" (47 of which are Hellenistic coarse ware), including Late Hellenistic grey ware and brown slipped ware. In this article survey finds in particular will be examined, because most of the Hellenistic material was found in the course of 2005 field survey. A major problem is that Iron Age and Hellenistic coarse wares are difficult to distinguish in this landscape.

Through the quantification of material and examination of clay there is a strong evidence to assume that the great majority of these vessels were manufactured in southwestern Paphlagonia. The local origin of the material, however, has not been proven by mineralogical analysis of the clay.

In our pottery study we have classified all the sherds earlier than the Roman period into a "Pre-Roman" category, forming 12 main groups from pre-Iron Age, Iron Age, Hellenistic and Late Hellenistic periods. Most of these pre-Roman groups are from Kimistene. Definable wares of these four periods are as follows: Pre-Iron Age sherds (from höyüks in the area), Iron Age grey ware, Iron Age painted ware, Iron Age coarse ware, Pontic skyphos fragment of northern Black Sea origin ( $4^{\text {th }}$ cent. B.C.), Hellenistic painted ware, Hellenistic relief ware, Hellenistic burnished ware, red-painted Kepez group, Late Hellenistic-Early Roman grey ware, Late HellenisticEarly Roman brown-slipped ware and Hellenistic coarse ware. Finds in local museums such as those in the Museum of Çankırı, which are mostly from graves in the area, have also been considered for our study (fig. 5). 


\section{CATALOGUE: I. PRE-IRON AGE POTTERY (PL. 1, NOS. 1-20)}

In the area surrounding Hadrianopolis, especially in the southern part of Eskipazar, there are some höyük sites on the plain where we have collected Chalcolithic and Bronze Age sherds. A few prehistoric sherds were also found in Hadrianopolis. These few pieces show a very different character to later Iron Age sherds. They have a coarse character without any distinctive forms and they were used for daily kitchen functions. Their characteristics are similar to those of pottery in Central Anatolia.

Our surveys were not able to provide a detailed and full presentation of the Pre-Iron Age material in Paphlagonia. Some general conclusions, however, can still be made. No Palaeolithic or Neolithic material was recognized among the finds, and this is more or less consistent with the rest of the northern Anatolia: this may be due to the mountainous character of the north and its relatively harsher climatic conditions. Two höyük sites in our survey region in Eskipazar, Tamışlar as well as Kutlukuyu Höyük near Ortaköy (Kuzupınar District), might be interpreted as small villages, hamlets, or isolated farmsteads.

19 of 36 sherds belong to open and 17 to closed forms; dimensions of forms should be very large. Their clay is yellowish red (5YR 5/6-4/6), reddish brown (5YR 4/4-5/4), brown (7.5YR 5/44/4-4/3, 10YR 5/3), red (2.5YR 4/6-5/6), very dark grey (7.5YR 3/1, 10YR 3/1, 2.5Y 3/1), light brown (7.5YR 6/4), reddish yellow (5YR 6/6), greyish brown (10YR 5/2), black (10YR 2/1), light yellowish brown (10YR 6/4), dark brown (7.5YR 3/2) and grey (10YR 5/1). Because of the low firing technique, there are some multicoloured samples in black (Gley $12.5 / \mathrm{N}, 7.5 \mathrm{YR} 2.5 / 1$ ) and grey $(7.5$ YR 3/1, 5YR 3/1, Gley $13 / \mathrm{N})$. Most of them were underfired.

Stone, sand, lime, grit and mica are the main inclusions. Most of them have a thick wall and were made on a slow wheel. Most of them are unslipped. Their surfaces were shaped with hands roughly. The determined slip colours are red (2.5YR 5/6-4/6-5/8, 10R 4/6), brown (7.5YR 4/3-5/4-4/2-5/2, 5/3-4/4, 10YR 5/3), reddish brown (2.5YR 4/4, 5YR 4/4-4/3-5/4), black ( Gley $12.5 / \mathrm{N}$ ), yellowish red (5YR 5/6), light brown (7.5YR 6/3-6/4), very dark grey (7.5YR 3/1, 10YR 3/1), pale brown (10YR 6/3), reddish yellow (5YR 7/6), dark reddish grey (5YR 4/2) and dark greyish brown (10YR 4/2). Same slip technique has been determined at a hollow bowl from the Mount II of İkiztepe (Alkım, Alkım and Bilgi 2003, 66, pl. CXXXIII, no. 205) which is an Early Bronze Age vessel.

On few sherds there is some external ornamentation. Decoration is observed on the body. Stamped, grooved and ridge decoration are the only patterns, as seen on nos. 9 and 19 with grooved decoration. On no. 16 there is a tworowed ridge decoration. Parallels to no. 16 are known from Gence Höyük near the village of Bezirhane in the Gölbaşı District of Ankara Province; they are dated to Middle Bronz Age (Omura 2007, 48, fig. 47:12). Similar ones are a Middle Bronz Age sherd from Höyük-Durupınar in Ankara (Omura 2006, 70, fig. 82:11); and a Late Bronz Age sherd from Külhöyük, in Ankara (Omura 2007, 49, fig. 62:13).

OPEN FORMS (pl. 1, nos. 1-4) / Rim Fragments (pl. 1, nos. 1-2)

An open form with a thickened and rounded slightly outcurved rim. There are some burning traces on its exterior surface. Both external and interior surfaces were burnished.

1. (No. 1212): Rim fragment; Kimistene, summit of the Acropolis, southern slope, found in 2005. pl. 1/1 and pl. 26/1.

Max. h 3.4 cm., d of rim $23.0 \mathrm{~cm}$., max. w 7.9 
cm., max. th $0.9 \mathrm{~cm}$.

Brown (10YR 5/3) slip on exterior, yellowish red (5YR 5/6) slip on interior. All of the surface is burnished. Hard, non-porous, yellowish red (5YR 5/6) and brown (10YR 5/3) fabric with frequent sand and tiny lime inclusions.

2. (No. 723): Rim fragment; Kimistene, Acropolis, temple's terrace, illegally excavated area under the temple's podium, surface find, found in 2005. pl. 1/2 and pl. 26/2.

Max. h 5.7 cm., max. w $5.5 \mathrm{~cm}$., max. th 1.0 $\mathrm{cm}$.

Pale brown (10YR 6/3) and dark grey (10YR 4/1) slip on exterior and interior rim, brown (7.5YR 5/2) slip on lower interior. Exterior surface is burnished. Hard, very sparsely porous, fired to brown (7.5YR 4/3) and dark grey (Gley $14 / \mathrm{N}$ ) fabric with frequent lime and sand inclusions.

Parallels: Özdoğan/Marro/Tibet 1999, 223, 225, drawing 1:6, drawing 2: Eylekderesi (12) and Gavurevleri (4).

Base Fragments of Open Forms (pl. 1, nos. 3-4)

Two fragments in plain and low base form.

3. (No. 1296): Base fragment; Kimistene, Acropolis, temple slope, illegal excavation area, found in 2005. pl. 1/3 and pl. 26/3. Max. h 4.0 cm., max. w 7.8 cm., max. th 1.3 $\mathrm{cm}$.

Black (Gley 1 2.5/N) slip on exterior; light brown (7.5YR 6/3) slip on interior. Average hardness; very sparsely porous, light brown (7.5YR 6/4) and black (Gley $12.5 / \mathrm{N}$ ) fabric with frequent lime and large grit inclusions.

4. (No. 663): Base fragment; Kimistene, Cistern, eastern slope, found in 2005. pl. 1/4 and pl. 26/4.
Max. h 1.7 cm., d of base 10.4 cm., max. w $5.2 \mathrm{~cm}$., max. th $1.1 \mathrm{~cm}$.

Red (2.5YR 4/6) slip on exterior; reddish yellow (5YR 6/6) unslipped surface on interior. Average hardness; non-porous, red (2.5YR $4 / 6$ ) and black (7.5YR 2.5/1) fabric with frequent lime and large grit inclusions.

Body Fragments of Open Forms (pl. 26, nos. 5-19)

Some pieces (nos. 6, 12 and 18) were polished on the exterior. In some fragments the interior face is flattened.

5. (No. 675): Body fragment; Kimistene, Cistern, eastern slope, found in 2005. pl. 26/5.

Max. h $2.2 \mathrm{~cm}$., max. w $2.6 \mathrm{~cm}$., max. th 0.9 $\mathrm{cm}$.

Red (10R 4/6) slip on exterior; red (2.5YR 4/6) slip on interior. Average hardness; very sparsely porous, red (2.5YR 4/6) and very dark grey (7.5YR 3/1) fabric with frequent lime inclusions.

6. (No. 730): Body fragment; Kimistene, Acropolis, temple's terrace, illegally excavated area under the temple's podium, found in 2005. pl. 26/6.

Max. h $2.5 \mathrm{~cm}$., max. w $2.4 \mathrm{~cm}$., max. th 0.7 $\mathrm{cm}$.

Dark reddish grey (5YR 4/2) slip on exterior; very dark grey (7.5YR 3/1) slip on interior. Exterior surface is burnished. Average hardness; non-porous, fine, reddish brown (5YR 4/4) fabric with some micaceous inclusions.

7. (No. 1357): Body fragment; Kimistene, Acropolis, eastern slope, found in 2005. pl. 26/7.

Max. h $3.0 \mathrm{~cm}$., max. w $3.9 \mathrm{~cm}$., max. th 1.2 $\mathrm{cm}$.

Reddish brown (2.5YR 4/4) slip on exterior 
and brown (7.5YR 4/3) slip on interior. Average hardness; very sparsely porous, fired to reddish brown (5YR 4/4) and very dark grey (7.5YR 3/1) fabric with frequent lime and some micaceous inclusions.

8. (No. 678): Body fragment; Kimistene, Cistern, eastern slope, found in 2005. pl. 26/8.

Max. h $3.5 \mathrm{~cm}$., max. w $4.1 \mathrm{~cm}$., max. th 1.0 $\mathrm{cm}$.

Red (2.5YR 5/6) slip on exterior and interior. Average hardness; non-porous, fired to yellowish red (5YR 5/6) fabric with frequent lime inclusions.

9. (No. 662): Body fragment; Kimistene, Acropolis, eastern slope, found in 2005. pl. 26/9.

Max. h $4.5 \mathrm{~cm}$., max. w $4.0 \mathrm{~cm}$., max. th 1.1 $\mathrm{cm}$.

Red (2.5YR 5/6) slip on exterior and interior. Average hardness; non-porous reddish yellow (5YR 6/6) and black (7.5YR 2.5/1) fabric with some lime inclusions.

10. (No. 483): Body fragment; Kimistene, Acropolis, southern slope, just below the summit, up to Deresemail creek, found in 2005. pl. 26/10.

Max. h $3.4 \mathrm{~cm}$., max. w $5.1 \mathrm{~cm}$., max. th 0.9 $\mathrm{cm}$.

Brown (7.5YR 4/3) slip on exterior; reddish brown (5YR 4/4) slip on interior. All of the surface is burnished. Hard, non-porous reddish yellow (5YR 6/6) and very dark grey (Gley $13 / \mathrm{N}$ ) fabric with frequent lime and medium grit inclusions.

11. (No. 660): Body fragment; Kimistene, Cistern, eastern slope, found in 2005. pl.

26/11.

Max. h $3.2 \mathrm{~cm}$., max. w $4.5 \mathrm{~cm}$., max. th 1.5 $\mathrm{cm}$.
Yellowish red (5YR 5/6) slip on exterior and interior. Average hardness; very sparsely porous, brown (7.5YR 4/3) fabric with frequent lime and grit inclusions.

12. (No. 1348): Body fragment; Kimistene, Acropolis, eastern slope, found in 2005. pl. 26/12.

Max. h $4.5 \mathrm{~cm}$., max. w $4.0 \mathrm{~cm}$., max. th 1.2 $\mathrm{cm}$.

Reddish brown (5YR 4/4) slip on exterior and brown (7.5YR 5/4) slip on interior. Exterior surface is burnished. Average hardness; nonporous, fine, brown (7.5YR 5/4) fabric with some tiny lime and sand inclusions.

13. (No. 1340): Body fragment; Kimistene, Cistern, eastern slope, found in 2005. pl. 26/13.

Max. h $4.5 \mathrm{~cm}$., max. w $3.6 \mathrm{~cm}$., max. th 1.1 $\mathrm{cm}$.

Red (2.5YR 4/6) slip on exterior and red (2.5YR 5/6) slip on interior. Average hardness; non-porous, red (2.5YR 5/6) and black (7.5YR 2.5/1) fabric with frequent lime inclusions.

14. (No. 674): Body fragment; Kimistene, Acropolis, eastern slope, found in 2005. pl. 26/14.

Max. h 4.7 cm., max. w 3.4 cm., max. th 1.1 $\mathrm{cm}$.

Red (2.5YR 5/8) slip on exterior and interior. Average hardness; very sparsely porous, fine, reddish brown (5YR 5/4) fabric with frequent lime and sand inclusions.

15. (No. 622): Body fragment; Kimistene, Acropolis, found in 2005. pl. 26/15.

Max. h $5.5 \mathrm{~cm}$., max. w $4.7 \mathrm{~cm}$., max. th 1.4 $\mathrm{cm}$.

Reddish brown (5YR 4/4) slip on exterior and red (2.5YR 5/6) slip on interior. Soft, non-po- 
rous, fine, yellowish red (5YR 4/6) fabric with frequent tiny lime and large grit inclusions.

16. (No. 626): Body fragment; Kimistene, Acropolis, found in 2005. pl. 26/16.

Max. h $4.8 \mathrm{~cm}$., max. w $6.7 \mathrm{~cm}$., max. th 1.3 $\mathrm{cm}$.

Reddish brown (2.5YR 4/4) slip on exterior; brown (7.5YR 4/3) slip on interior. Soft, very sparsely porous, reddish brown (5YR 4/4) and very dark grey (7.5YR 3/1) fabric with frequent lime and large grit inclusions.

17. (No. 659): Body fragment; Kimistene, Cistern, eastern slope, found in 2005. pl. 26/17.

Max. h 8.1 cm., max. w 4.4 cm., max. th 1.7 $\mathrm{cm}$.

Light brown (7.5YR 6/4) unslipped surface on exterior; brown (7.5YR 5/4) slip on interior. Average hardness; non-porous, fine, reddish brown (5YR 4/4) fabric with frequent tiny lime and large grit inclusions.

18. (No. 658): Body fragment; Kimistene, Cistern, eastern slope, found in 2005. pl. 26/18.

Max. h 7.5 cm., max. w $7.3 \mathrm{~cm}$., max. th 1.4 $\mathrm{cm}$.

Reddish brown (5YR 4/3) slip on exterior; brown (7.5YR 5/4) slip on interior. Exterior surface is burnished. Average hardness; nonporous, fine, brown (7.5YR 4/4) fabric with frequent tiny lime and some sand inclusions.

19. (No. 687): Body fragment; Kimistene, Cistern, eastern slope, found in 2005. pl. 26/19.

Max. h 8.3 cm., max. w 7.4 cm., max. th 1.1 $\mathrm{cm}$.

Red (2.5YR 5/6) slip on exterior and interior. Average hardness; non-porous, fired to reddish yellow (5YR 6/6) and light yellowish brown (10YR 6/4) fabric with frequent large grit inclusions.

\section{CLOSED FORMS (pl. 1, no. 20)}

Most of these forms could be cooking wares.

Rim Fragment of a Closed Form (pl. 1, no. 20)

20. (No. 1115): Rim fragment; Kimistene, Acropolis, found in 2005. pl. 1/20 and pl. 27/20.

Max. h $5.0 \mathrm{~cm}$., max. w $4.4 \mathrm{~cm}$., max. th $1.1 \mathrm{~cm}$.

Black (Gley 1 2.5/N) slip on exterior; black (Gley 1 2.5/N) unslipped surface on interior. Exterior surface is burnished. Average hardness; very sparsely porous, fine, black (10YR 2/1) fabric with frequent large grit and some lime inclusions.

Body Fragments of Closed Forms (pls. 27-28, nos. 21-35)

Some sherds (nos. 23, 25-26, 29, 33 and $34)$ were polished on the exterior. On some fragments the interior face is flattened.

21. (No. 861): Body fragment; Kimistene, Acropolis, temple slope, illegally excavated pit at the altar, found in 2005. pl. 27/21.

Max. h $3.0 \mathrm{~cm}$., max. w $3.2 \mathrm{~cm}$., max. th 1.0 $\mathrm{cm}$.

Brown (7.5YR 4/2) slip on exterior; pale brown (10YR 6/3) unslipped surface on interior. Exterior surface is burnished. Average hardness; non-porous, brown (10YR 5/3) and very dark grey (Gley $13 / \mathrm{N}$ ) fabric with some sand, minor grit and lime inclusions. 
22. (No. 1335): Body fragment; Kimistene, Cistern, eastern slope, found in 2005. pl. 27/22.

Max. h 4.4 cm., max. w 4.5 cm., max. th 1.3 $\mathrm{cm}$.

Red (2.5YR 4/6) slip on exterior; grey (10YR $5 / 1$ ) unslipped surface on interior. Average hardness; non-porous, yellowish red (5YR $5 / 6)$ and very dark grey (7.5YR 3/1) fabric with some lime and sand inclusions.

23. (No. 700): Body fragment; Kimistene, Acropolis, temple slope, eastern part, found in 2005. pl. 27/23.

Max. h $4.3 \mathrm{~cm}$., max. w $4.0 \mathrm{~cm}$., max. th 1.2 $\mathrm{cm}$.

Light brown (7.5YR 6/4) slip on exterior; reddish yellow (7.5YR 6/6) unslipped surface on interior. Hard, non-porous, fine, reddish yellow (5YR 6/6) fabric with frequent tiny lime inclusions.

24. (No. 722): Body fragment; Kimistene, Acropolis, temple's terrace, illegally excavated area under the temple's podium, found in 2005. pl. 27/24.

Max. h $4.6 \mathrm{~cm}$., max. w $5.0 \mathrm{~cm}$., max. th 1.3 $\mathrm{cm}$.

Very dark grey (Gley $13 / \mathrm{N}$ ) unslipped surface on exterior; pale brown (10YR 6/3) slip on interior. Average hardness; non-porous, greyish brown (10YR 5/2) some sand, large grit and lime inclusions.

25. (No. 485): Body fragment; Kimistene, Acropolis, southern slope, just below the summit, up to Deresemail creek, found in 2005. pl. 27/25.

Max. h $4.5 \mathrm{~cm}$., max. w $5.3 \mathrm{~cm}$., max. th 1.2 $\mathrm{cm}$.

Brown (7.5YR 4/3) slip on exterior; brown (7.5YR 5/4) unslipped surface on interior. Exterior surface is burnished. Soft, non-porous, fine, brown $(7.5 Y R$ 4/3) fabric with frequent tiny lime inclusions.

26. (No. 706): Body fragment; Kimistene, Acropolis, temple slope, eastern part, found in 2005. pl. 27/26.

Max. h $4.8 \mathrm{~cm}$., max. w $5.2 \mathrm{~cm}$., max. th 1.3 $\mathrm{cm}$.

Brown (7.5YR 4/4) slip on exterior; light yellowish brown (10YR 6/4) unslipped surface on interior. Exterior surface is burnished. Soft, non-porous, fine, yellowish red (5YR $5 / 6)$ fabric with frequent medium grit and lime inclusions.

27. (No. 679): Body fragment; Kimistene, Cistern, eastern slope, found in 2005. pl. 27/27.

Max. h 5.7 cm., max. w 4.5 cm., max. th 2.1 $\mathrm{cm}$.

Reddish brown (2.5YR 4/4) slip on exterior; brown (7.5YR 5/4) unslipped surface on interior. Soft, non-porous, reddish brown (5YR $4 / 4$ ) and very dark grey (5YR 3/1) fabric with frequent tiny lime and medium grit inclusions.

28. (No. 719): Body fragment; Kimistene, Acropolis, found in 2005. pl. 27/28.

Max. h $6.3 \mathrm{~cm}$., max. w $4.4 \mathrm{~cm}$., max. th 1.0 $\mathrm{cm}$.

Brown (7.5YR 5/3) slip on exterior; dark grey (Gley $14 / \mathrm{N}$ ) unslipped surface on interior. Average hardness; very sparsely porous, very dark grey (2.5Y 3/1) fabric with some sand and lime inclusions.

29. (No. 1294): Body fragment; Kimistene, Acropolis, temple slope, illegally excavated pit, found in 2005. pl. 27/29.

Max. h $5.5 \mathrm{~cm}$., max. w $5.5 \mathrm{~cm}$., max. th 1.3 $\mathrm{cm}$.

Black (Gley 1 2.5/N) slip on exterior; light brown (7.5YR 6/4) unslipped surface on in- 
terior. Exterior surface is burnished. Average hardness; very sparsely porous, fired to light brown (7.5YR 6/4) and black (Gley $12.5 / \mathrm{N})$ fabric with infrequent tiny lime and medium grit inclusions.

30. (No. 672): Body fragment; Kimistene, Cistern, eastern slope, found in 2005. pl. 27/30.

Max. h $5.3 \mathrm{~cm}$., max. w $6.3 \mathrm{~cm}$., max. th 1.6 $\mathrm{cm}$.

Reddish brown (2.5YR 4/4) slip on exterior; yellowish red (5YR 5/6) unslipped surface on interior. Average hardness; sparsely porous, fired to yellowish red (5YR 4/6) and yellowish brown (10YR 5/4) fabric with frequent tiny lime, some large grit and sand inclusions.

31. (No. 670): Body fragment; Kimistene, Cistern, eastern slope, found in 2005. pl. 27/31.

Max. h 5.8 cm., max. w $5.5 \mathrm{~cm}$., max. th 1.1 $\mathrm{cm}$.

Red (10R 4/6) slip on exterior; grey (7.5YR 5/1) unslipped surface on interior. Average hardness; very sparsely porous, fired to very dark grey (10YR 3/1) fabric with frequent lime and medium grit inclusions.

32. (No. 664): Body fragment; Kimistene, Cistern, eastern slope, found in 2005. pl. 27/32.

Max. h 6.7 cm., max. w $5.5 \mathrm{~cm}$., max. th 1.0 $\mathrm{cm}$.

Red (2.5YR 4/6) slip on exterior; brown (7.5YR 5/4) unslipped surface on interior. Soft, non-porous dark brown (7.5YR 3/2) fabric with some medium grit and lime inclusions.

33. (No. 725): Body fragment; Kimistene, Acropolis, temple's terrace, illegally excavated area under the temple's podium, found in
2005. pl. 27/33.

Max. h $6.5 \mathrm{~cm}$., max. w $5.2 \mathrm{~cm}$., max. th 0.9

$\mathrm{cm}$.

Dark greyish brown (10YR 4/2) slip on exterior; brown (7.5YR 5/4) unslipped surface on interior. Exterior surface is burnished. Soft, non-porous, fired to very dark grey $(7.5 \mathrm{YR}$ $3 / 1$ ) fabric with frequent large grit, micaceous and lime inclusions.

34. (No. 973): Body fragment; Kimistene, Cistern, eastern slope, found in 2005. pl. 27/34.

Max. h $6.0 \mathrm{~cm}$., max. w $8.0 \mathrm{~cm}$., max. th 1.0 $\mathrm{cm}$.

Very dark grey (10YR 3/1) slip on exterior; brown (7.5YR 5/3) unslipped surface on interior. Exterior surface is burnished. Hard, very sparsely porous, grey (10YR 5/1) fabric with some sand and lime, rare micaceous inclusions.

35. (No. 1119): Body fragment; Kimistene, Acropolis, found in 2005. pl. 28/35.

Max. h $8.1 \mathrm{~cm}$., max. w $7.8 \mathrm{~cm}$., max. th 1.4 $\mathrm{cm}$.

Reddish brown (5YR 5/4) slip on exterior; brown (7.5YR 4/3) unslipped surface on interior. Soft, non-porous, fired to very dark grey (7.5YR 3/1) fabric with frequent large grit, micaceous and lime inclusions.

36. (No. 630): Body fragment; Kimistene, Acropolis, found in 2005. pl. 28/36.

Max. h 6.9 cm., max. w 10.0 cm., max. th 1.3 $\mathrm{cm}$.

Red (10R 4/6) slip on exterior; light yellowish brown (10YR 6/4) unslipped surface on interior. Hard, non-porous, fired to red $(2.5 \mathrm{YR}$ 4/6) and black (7.5YR 2.5/1) fabric with some large grit and tiny lime inclusions. 
II. IRON AGE GREY WARE (pls. 1-2, nos. 37-56)

Iron Age grey ware is often associated with a Phrygian presence in Central Anatolia. The major concentration of "Phrygian" grey ware is west-central Anatolia. This fabric endures through the Middle and Late Iron Ages and into the Hellenistic and even Roman periods. A sample of grey vessels from the new excavations at Gordion has been subjected to chemical analyses, which confirmed that the same clay was used for virtually all of the vessels in the Iron Age corpus, and perhaps this situation did not change in the Hellenistic period (Stewart 2010, 147; Henrickson 2005, 125).

Grey ware is the most distinctive Iron Age group among the southwestern Paphlagonian finds. Matthews's surveys produced numerous examples of grey ware from Inner Paphlagonia, for instance from PS 178, Höyük Tepesi, in the Eldivan Plain (Matthews 2009, 153, fig. 5.3, no. 5, site PS 178 and 155, fig. 5.5, PS 052-Kızılca Tepe). The presence of this ware in Inner Paphlagonia was interpreted by Matthews as it generates the northern limits of the distribution of Phrygian heritage (Matthews 2009, 154.).

Its clay is mostly dark grey $(7.5 \mathrm{YR} 4 / 1$, 10YR 4/1, 2.5Y 4/1, Gley $14 / \mathrm{N})$, grey (2.5Y 5/1$6 / 1$, Gley $15 / \mathrm{N}-6 / \mathrm{N})$, black $(2.5 \mathrm{Y} 2.5 / 1,10 \mathrm{YR}$ $2 / 1$, Gley $12.5 / \mathrm{N})$ and very dark grey (2.5Y $3 / 1$, Gley $13 / \mathrm{N}$ ). Grey fabric and polished black slip are both firmly in the Phrygian ceramic tradition (Stewart 2010, 210).

Lime, mica, sand and grit were used as inclusions in minor proportions, in some samples at a dimension of 10-20 mm. It has a hard and non-porous clay with a very dark grey $(2.5 \mathrm{Y} 3 / 1$, Gley $13 / \mathrm{N})$, dark gary (10YR 4/1, $2.5 \mathrm{Y} 4 / 1$ ), black (Gley $12.5 / \mathrm{N})$, grey (2.5Y 5/1-6/1, Gley 1 $5 / \mathrm{N})$, light grey (5YR 7/1) slip. In some fragments their surface is polished. Their thickness differs between 4 and $10 \mathrm{~mm}$. On samples nos. 37-38, 40, 51,57 and 63 polishing provided a metallic sheen on the surface. Almost no ornamentation has been applied.

Functionally they should be serving vessels, but they could also be associated with some religious purposes and used as ritual vessels. This specific type of ware bears no decoration; their grey or blackish fabric is fine, with minute mineral inclusions in medium sizes, and a burnished soapy texture to the surface, often with a "silvery sheen". One major vessel form is a bowl of $c .20$ $\mathrm{cm}$ diameter which is clearly an imitation of metallic forms.

In 2005 around 37 fragments were collected, 15 of which belonged to open and 22 to closed forms. Most of the sherds are from the hilltop sanctuary at Kimistene: 22 fragments from the illegally excavated area in the temenos of Kimistene, 3 of them from the Acropolis, 7 from the southern slope, 2 from the western slope, 1 from the northwestern slope, 1 from the eastern slope, and 1 from the Cistern. Sherds found in the illegal pit in the temenos could be indications of a fire that occured in the stratified levels beneath the temple's podium.

The date of Iron Age grey ware should be Late Middle Iron Age and Late Iron Age, i.e. from the beginning of the 7th to mid 4th centuries B.C. G. D. Tetova's analysis of the material at Gordion reveals that the Early Phrygian ceramic tradition continued well into the 4th century B.C., with about $90 \%$ of the vessel forms based on prototypes established in the 9th century or earlier (Stewart 2010, 49-50; Toteva 2007, 53, 59.). Thus the forms remain unchanged for many centuries. That is the reason why grey ware from southwestern Paphlagonia is difficult to date through analogy with some other sites. Radiocarbon dates at Boğazköy put the Büyükkaya-Stufe into the 9th century B.C., while the Büyükkale IIa-b levels appear to date to the 8th century B.C. and have material comparable to that from Maşat III-II, Kültepe Iron Age and Kaman-Kalehöyük IIc-a (Matthews 2009, 153; and Genz 2004a: table 6). 
It is interesting to note that the whole-mouth jars and craters found in Matthews's surveys were not recovered at Kimistene.

OPEN FORMS (pls. 1-2, nos. 37-47)

Most of them were polished in their interior surface.

Rim Fragments of Open Forms (pls. 1-2, nos. 37-46)

Most of them have straight walls with outcurved rims. Whole surfaces of nos. 37-38, 40, $42-43$, and 45 , interior surface of no. 44 and exterior surface of nos. 46 and 57 are polished.

37. (No. 745): Rim fragment; Kimistene, Acropolis, temple's terrace, illegally excavated area under the temple's podium, found in 2005. pl. 1/37 and pl. 28/37.

Max. h 1.4 cm., max. w $2.1 \mathrm{~cm}$., max. th 0.5 $\mathrm{cm}$.

Very dark grey (Gley $13 / \mathrm{N}$ ) slip on all of surface. Exterior and interior surface are burnished. Average hardness; thin paste, non-porous, fine, dark grey (Gley $14 / \mathrm{N}$ ) fabric with frequent tiny lime inclusions.

38. (No. 746): Rim fragment; Kimistene, Acropolis, temple's terrace, illegally excavated area under the temple's podium, found in 2005. pl. 1/38 and pl. 28/38.

Max. h $1.7 \mathrm{~cm}$., max. w $2.9 \mathrm{~cm}$., max. th 0.4 $\mathrm{cm}$.

Very dark grey (Gley $13 / \mathrm{N}$ ) slip on exterior, grey $($ Gley $15 / \mathrm{N})$ slip on interior. Exterior and interior surface are burnished. Average hardness; thin paste, non-porous, fine, grey (Gley $15 / N$ ) fabric with some tiny lime inclusions.

39. (No. 717): Rim fragment; Kimistene,
Acropolis, temple's terrace, illegally excavated area under the temple's podium, found in 2005. pl. 28/39.

Max. h $2.1 \mathrm{~cm}$., max. w $2.7 \mathrm{~cm}$., max. th 0.8 $\mathrm{cm}$.

Very dark grey (Gley $13 / \mathrm{N}$ ) slip on exterior and interior, hard, very sparsely porous, fine, black (10YR 2/1) fabric with frequent tiny lime and some sand inclusions.

40. (No. 860): Rim fragment; Kimistene, Acropolis, temple's terrace, illegally excavated area under the temple's podium, found in 2005. pl. 1/40 and pl. 28/40.

Max. h $3.8 \mathrm{~cm}$., max. w $3.5 \mathrm{~cm}$., max. th 0.5 $\mathrm{cm}$.

Very dark grey (Gley $13 / \mathrm{N}$ ) slip on all of surface. Exterior and interior surface are burnished. Average hardness; non-porous, fired to very dark grey $(2.5 \mathrm{Y} 3 / 1)$ fabric with tiny lime and rare micaceous inclusions.

41. (No. 1310): Rim fragment; Kimistene, Acropolis, southern slope, found in 2005. pl. 1/41 and pl. 28/41.

Max. h $1.2 \mathrm{~cm}$., d of rim $10.4 \mathrm{~cm}$., max. w 4.3 cm., max. th $0.5 \mathrm{~cm}$.

Light grey (5YR 7/1) slip on exterior and interior. All of surface is burnished. Hard, nonporous, fired to reddish grey (5YR 5/2) and dark grey $(7.5 \mathrm{YR} 4 / 1)$ fabric with some tiny lime inclusions.

42. (No. 1210): Rim fragment; Kimistene, summit of the Acropolis, southern slope, found in 2005. pl. 1/42 and pl. 28/42.

Max. h 3.8 cm., d of rim $26.6 \mathrm{~cm}$., max. w 6.0 cm., max. th $1.2 \mathrm{~cm}$.

Very dark grey (Gley $13 / \mathrm{N}$ ) slip on exterior and interior, hard, non-porous, fine, dark grey (Gley $14 / \mathrm{N}$ ) fabric with frequent tiny lime inclusions.

Parallel: Matthews 2009, 162, fig. 5.14/10 
(from PS 015). Its paste is pale brown (10 YR $6 / 3$ ) and its exterior is burnished.

43. (No. 1118): Rim fragment; Kimistene, Acropolis, found in 2005. pl. 1/43 and pl. 28/43.

Max. h 2.7 cm., d of rim $15.0 \mathrm{~cm}$., max. w 3.5 cm., max. th $0.9 \mathrm{~cm}$.

Black (Gley 1 2.5/N) slip on all of surface. Exterior and interior surface are burnished. Hard, non-porous, fine, black (10YR 2/1) fabric with frequent lime inclusions.

Parallel: Özdoğan/Marro/Tibet 1999, 223, Gavurevleri drawing 5/3 [from the Village Yazıcımeydanı (Meydanköy), GavurevleriHöyük, Gavurevleri/Ortaboy Höyük in Province Kastamonu].

44. (No. 884): Rim fragment; Kimistene, Acropolis, southern slope, underground cave, surface find, found in 2005. pl. 1/44 and pl. 28/44.

Max. h 2.8 cm., d of rim $33.6 \mathrm{~cm}$., max. w 6.1 cm., max. th $1.1 \mathrm{~cm}$.

Light brownish grey (2.5Y 6/2) unslipped surface on exterior, dark grey $(2.5 \mathrm{Y} 4 / 1)$ abrasion slip interior rim. Interior surface is burnished. Hard, very sparsely porous, fine, grey (Gley $16 / \mathrm{N}$ ) fabric with occasional sand inclusions. Parallels: Matthews 2009, 153, 168, fig. 5.17/6 (from the site PS178); Özdoğan/Marro/ Tibet 1997, 309, drawing 6 (from the Village Samanlı̈ren, Yüklütepe, Yamaç Settlement in Taşköprü); and Omura 2008, 51, fig. 106/5 (from Devecigeçidi, in District Çelebi, Village Karabucak, Province Kırşehir; Late Iron Age).

45. (No. 737): Rim fragment; Kimistene, Acropolis, temple's terrace, illegally excavated area under the temple's podium, found in 2005. pl. 2/45 and pl. 28/45.

Max. h $2.5 \mathrm{~cm}$., d of rim $36.8 \mathrm{~cm}$., max. w 5.6 cm., max. th $1.3 \mathrm{~cm}$.
Very dark grey (Gley $13 / \mathrm{N}$ ) slip on exterior and interior. All of surface is burnished. Hard, non-porous, fine, grey $(2.5 \mathrm{Y} 5 / 1)$ fabric with some large grit inclusions.

46. (No. 393): Rim fragment; Kimistene, Acropolis, temple terrace, northwestern slope, found in 2005. pl. 2/46 and pl. 28/46.

Max. h 4.2 cm., d of rim 23.4 cm., max. w 8.2 cm., max. th $1.1 \mathrm{~cm}$.

Black (Gley 1 2.5/N) slip on exterior, grey $(2.5 \mathrm{Y} 5 / 1)$ slip on interior. Exterior surface is burnished. Hard, very sparsely porous, fired to grey (2.5Y 5/1) and black (2.5Y 2.5/1) fabric with some tiny lime and sand inclusions.

Base Fragment of an Open Form (pl. 2, no. 47)

This group contains rounded flat bases.

47. (No. 1359): Base fragment; Kimistene, Cistern, eastern slope, found in 2005. pl. 2/47 and $\mathrm{pl} . \mathbf{2 8} / \mathbf{4 7}$.

Max. h $2.6 \mathrm{~cm}$., d of base $8.2 \mathrm{~cm}$., max. w 7.8 cm., max. th $0.8 \mathrm{~cm}$.

Reddish brown (2.5YR 4/4) slip on exterior, brown (7.5YR 4/4) slip on interior. All of surface is burnished. Hard, very sparsely porous, grey (Gley $15 / \mathrm{N}$ ) fabric with frequent lime and micaceous inclusions.

Body Fragments of Open Forms (pl. 28, nos. 48-51)

48. (No. 716): Body fragment; Kimistene, Acropolis, temple's terrace, illegally excavated area under the temple's podium, found in 2005. pl. 28/48.

Max. h $2.3 \mathrm{~cm}$., max. w $1.7 \mathrm{~cm}$., max. th 1.0 $\mathrm{cm}$.

Very dark grey (Gley $13 / \mathrm{N}$ ) slip on all of surface. Exterior surface is burnished. Average 
hardness; non-porous dark grey (10YR 4/1) fabric with some tiny lime, sand and medium grit inclusions.

49. (No. 743): Body fragment; Kimistene, Acropolis, temple's terrace, illegally excavated area under the temple's podium, found in 2005. pl. 28/49.

Max. h $3.3 \mathrm{~cm}$., max. w $2.7 \mathrm{~cm}$., max. th 0.7 $\mathrm{cm}$.

Very dark grey $(2.5 \mathrm{Y} 3 / 1)$ slip on exterior and interior. All of the surface is burnished. Average hardness; very sparsely porous, fine, very dark grey (Gley $13 / \mathrm{N}$ ) fabric with some tiny lime inclusions.

50. (No. 721): Body fragment; Kimistene, Acropolis, temple's terrace, illegally excavated area under the temple's podium, found in 2005. pl. 28/50.

Max. h 2.8 cm., max. w $3.6 \mathrm{~cm}$., max. th 0.9 $\mathrm{cm}$.

Black (Gley $12.5 / \mathrm{N}$ ) slip on all over the surface. Exterior surface is burnished. Average hardness; non-porous, black (2.5Y 2.5/1) fabric with frequent tiny lime, sand and medium grit inclusions.

51. (No. 891): Body fragment; Kimistene, Acropolis, southern slope, underground cave, found in 2005. pl. 28/51.

Max. h 4.0 cm., max. w $4.7 \mathrm{~cm}$., max. th 1.1 $\mathrm{cm}$.

Dark grey (2.5Y 4/1) slip on exterior and interior. All over the surface is burnished. Hard, non-porous, dark grey (Gley $14 / \mathrm{N}$ ) fabric with some tiny lime and rare micaceous inclusions.

CLOSED FORMS (pl. 2, nos. 52-56) / Rim Fragments of Closed Forms (pl. 2, nos. 5255)
Most of them belong to the wider forms with folded rim.

52. (No. 1247): Rim fragment; Kimistene, western slope of the temenos; found in 2005.

pl. 2/52 and pl. 29/52.

Max. h $3.2 \mathrm{~cm}$., d of rim $10.0 \mathrm{~cm}$., max. w 5.3 cm., max. th $0.8 \mathrm{~cm}$.

Dark grey (Gley $14 / \mathrm{N}$ ) slip on exterior; grey (2.5Y 5/1) unslipped surface on interior. Hard, very sparsely porous, fine, dark grey $(2.5 \mathrm{Y}$ 4/1) fabric with some sand inclusions.

Parallel: Omura 1996, 245, 260, fig. 2 , 1 (from Karakaya Höyük in Kaman County, Province Kırşehir).

53. (No. 1219): Rim fragment; Kimistene, summit of the Acropolis, southern slope, found in 2005. pl. 2/53 and pl. 29/53.

Max. h 2.2 cm., d of rim 14.4 cm., max. w 3.8 cm., max. th $0.8 \mathrm{~cm}$.

Very dark grey (2.5Y 3/1) unslipped surface on exterior and interior. Soft, non-porous, black (2.5Y 2.5/1) fabric with frequent lime and sand inclusions.

Parallel: Omura 1996, 247, 263, fig. 5, 1 (from Boz Höyük in Polatlı County, Province Ankara).

54. (No. 1116): Rim fragment; Kimistene, Acropolis, found in 2005. pl. 2/54 and pl. 29/54.

Max. h $3.6 \mathrm{~cm}$., d of rim $29.6 \mathrm{~cm}$., max. w 6.7 cm., max. th $0.9 \mathrm{~cm}$.

Grey (2.5Y 5/1) unslipped surface on exterior; grey $(2.5 Y$ 6/1) unslipped surface on interior. Soft, non-porous, fine, dark grey $(2.5 \mathrm{Y} 4 / 1)$ fabric with occasional sand and tiny lime inclusions.

55. (No. 927): Rim fragment; Kimistene, Acropolis, found 2005. pl. 2/55 and pl. 29/55. Max. h $3.2 \mathrm{~cm}$., d of rim $24.0 \mathrm{~cm}$., max. w 5.6 
cm., max. th $0.6 \mathrm{~cm}$.

Dark grey $(2.5 \mathrm{Y} 4 / 1)$ slip on exterior and interior. Soft, very sparsely porous, reddish brown (5YR 4/3) and dark grey (2.5Y 4/1) fabric with rare micaceous and large grit inclusions.

\section{Base Fragment of a Closed Form (pl. 2,} no. 56)

56. (No. 720): Base fragment; Kimistene, Acropolis, temple's terrace, illegally excavated area under the temple's podium. pl. 2/56 and pl. 29/56.

Max. h $1.3 \mathrm{~cm}$., d of rim $20.0 \mathrm{~cm}$., max. w 5.9 cm., max. th $0.8 \mathrm{~cm}$.

Very dark grey (Gley $13 / \mathrm{N})$ slip on exterior; dark grey (10YR 4/1) unslipped surface on interior. Average hardness; very sparsely porous, reddish brown (5YR 4/4) and very dark grey $(2.5 Y 3 / 1)$ fabric with frequent lime and rare grog inclusions.

Body Fragments of Closed Forms (pl. 29, nos. 57-73)

The exterior surface of nos. 57, 59, 63, 65, 67 and 69-73 were polished.

57. (No. 732): Body fragment; Kimistene, Acropolis, temple's terrace, illegally excavated area under the temple's podium, found in 2005. pl. 29/57.

Max. h $2.4 \mathrm{~cm}$., max. w $2.0 \mathrm{~cm}$., max. th 0.6 $\mathrm{cm}$.

Black (Gley 1 2.5/N) slip on exterior; grey (Gley $16 / \mathrm{N}$ ) unslipped surface on interior. Exterior surface is burnished. Hard, very sparsely porous, fine, grey (Gley $15 / \mathrm{N}$ ) fabric with some lime inclusions.

58. (No. 769): Body fragment; Kimistene, Acropolis, temple's terrace, illegally excavated area under the temple's podium, found in
2005. pl. 29/58.

Max. h $2.0 \mathrm{~cm}$., max. w $2.0 \mathrm{~cm}$., max. th 0.7 $\mathrm{cm}$.

Very dark grey (Gley $13 / \mathrm{N}$ ) unslipped surface on exterior; reddish brown (5YR 5/4) unslipped surface on interior. Average hardness; very sparsely porous, fired to dark reddish grey (5YR 4/2) and very dark grey (Gley $13 / \mathrm{N}$ ) fabric with frequent tiny lime and sand inclusions.

59. (No. 1333): Body fragment; Kimistene, summit of the Acropolis, southern slope, found in 2005. pl. 29/59.

Max. h $2.3 \mathrm{~cm}$., max. w $2.1 \mathrm{~cm}$., max. th 0.9 $\mathrm{cm}$.

Very dark grey (Gley $13 / \mathrm{N})$ slip on exterior; dark grey (Gley $14 / \mathrm{N}$ ) unslipped surface on interior. Exterior surface is burnished. Average hardness; very sparsely porous, fine, dark grey (Gley $14 / \mathrm{N}$ ) fabric with frequent large grit and some lime inclusions.

60. (No. 762): Body fragment; Kimistene, Acropolis, temple's terrace, illegally excavated area under the temple's podium, found in 2005. pl. 29/60.

Max. h $1.6 \mathrm{~cm}$., max. w $2.8 \mathrm{~cm}$., max. th 0.7 $\mathrm{cm}$.

Dark grey $(2.5 \mathrm{Y} 4 / 1)$ slip on exterior; black (Gley $12.5 / \mathrm{N}$ ) unslipped surface on interior. Soft, non-porous, fine, black (2.5Y 2.5/1) fabric with some minor grit inclusions.

61. (No. 753): Body fragment; Kimistene, Acropolis, temple's terrace, illegally excavated area under the temple's podium, found in 2005. pl. 29/61.

Max. h $2.8 \mathrm{~cm}$., max. w $3.2 \mathrm{~cm}$., max. th 0.9 $\mathrm{cm}$.

Very dark grey (2.5Y 3/1) slip on exterior; dark grey $(2.5 \mathrm{Y} 4 / 1)$ slip on interior. Average hardness; non-porous, black (2.5Y 2.5/1) 
fabric with some lime and medium grit inclusions.

62. (No. 646): Body fragment; Kimistene, Acropolis, western slope, found in 2005. pl. 29/62.

Max. h $2.3 \mathrm{~cm}$., max. w $2.8 \mathrm{~cm}$., max. th 0.9 cm.

Dark grey (Gley $14 / 1)$ unslipped surface on exterior and interior. Hard, non-porous, fine, dark grey (Gley $14 / \mathrm{N}$ ) fabric with no visible inclusions.

63. (No. 866): Rim fragment; Kimistene, Acropolis, temple slope, illegally excavated pit, found in 2005. pl. 29/63.

Max. h 2.9 cm., max. w $3.5 \mathrm{~cm}$., max. th 1.2 $\mathrm{cm}$.

Black (Gley 1 2.5/N) slip on exterior; very dark grey (Gley $13 / \mathrm{N}$ ) unslipped surface on interior. Exterior surface is burnished. Average hardness; very sparsely porous, fine, black (Gley $12.5 / \mathrm{N}$ ) fabric with frequent tiny lime and some large grit inclusions.

64. (No. 754): Body fragment; Kimistene, Acropolis, temple's terrace, illegally excavated area under the temple's podium, found in 2005. pl. 29/64.

Max. h 3.9 cm., max. w $3.1 \mathrm{~cm}$., max. th 1.0 $\mathrm{cm}$.

Very dark grey (Gley $13 / \mathrm{N}$ ) unslipped surface on exterior and interior, Average hardness; non-porous, fine, very dark greyish brown (10YR 3/2) fabric with some sand and micaceous inclusions.

65. (No. 735): Body fragment; Kimistene, Acropolis, temple's terrace, illegally excavated area under the temple's podium, found in 2005. pl. 29/65.

Max. h 3.9 cm., max. w 3.9 cm., max. th 0.5 $\mathrm{cm}$.
Very dark grey (Gley $13 / \mathrm{N}$ ) slip on exterior; light grey (2.5Y 7/1) unslipped surface on interior. Exterior surface is burnished. Average hardness; very sparsely porous, grey $(2.5 \mathrm{Y}$ $5 / 1$ ) fabric with frequent sand inclusions.

66. (No. 728): Body fragment; Kimistene, Acropolis, temple's terrace, illegally excavated area under the temple's podium, found in 2005. pl. 29/66.

Max. h $4.1 \mathrm{~cm}$., max. w $3.3 \mathrm{~cm}$., max. th 1.1 $\mathrm{cm}$.

Grey (2.5Y 6/1) slip on exterior; grey (2.5Y $5 / 1)$ unslipped surface on interior. Hard, nonporous, dark greyish brown (2.5Y 4/2) and grey $(2.5 \mathrm{Y} 6 / 1)$ fabric with frequent lime inclusions.

67. (No. 1414): Body fragment; Kimistene, Acropolis, temple slope, illegally excavated pit, layer no. 3, found in 2005. pl. 29/67.

Max. h $5.3 \mathrm{~cm}$., max. w $4.8 \mathrm{~cm}$., max. th 0.6 $\mathrm{cm}$.

Very dark grey (Gley $13 / \mathrm{N}$ ) slip on exterior; grey $(2.5 Y 5 / 1)$ unslipped surface on interior. Exterior surface is burnished. Average hardness; non-porous, fine, very dark grey $(2.5 \mathrm{Y}$ $3 / 1$ ) fabric with frequent sand and rare mica inclusions.

68. (No. 734): Body fragment; Kimistene, Acropolis, temple's terrace, illegally excavated area under the temple's podium, found in 2005. pl. 29/68.

Max. h $4.2 \mathrm{~cm}$., max. w $5.2 \mathrm{~cm}$., max. th 1.0 $\mathrm{cm}$.

Very dark grey (2.5Y 3/1) unslipped surface on exterior; dark grey (Gley $14 / \mathrm{N}$ ) unslipped surface on interior. Average hardness; very sparsely porous, fine, dark grey (2.5Y 4/1) fabric with frequent mica and tiny lime inclusions. 
69. (No. 494): Body fragment; Kimistene, Acropolis, southern slope, just below the summit, up to Deresemail creek, found in 2005.

pl. 29/69.

Max. h $4.0 \mathrm{~cm}$., max. w $6.1 \mathrm{~cm}$., max. th 0.7 $\mathrm{cm}$.

Very dark grey (Gley $13 / \mathrm{N})$ slip on exterior, grey $(2.5 Y$ 5/1) unslipped surface on interior. Exterior surface is burnished. Hard, non-porous, fired to dark grey $(2.5 \mathrm{Y} 4 / 1)$ and brown (7.5YR 5/4) fabric with frequent tiny lime, sand and rare micaceous inclusions.

70. (No. 699): Body fragment; Kimistene, Acropolis, temple slope, first eastern slope, found in 2005. pl. 29/70.

Max. h $6.7 \mathrm{~cm}$., max. w $6.6 \mathrm{~cm}$., max. th 0.7 $\mathrm{cm}$.

Dark grey $(2.5 \mathrm{Y} 4 / 1)$ slip on exterior; greyish brown (10YR 5/2) unslipped surface on interior. Exterior surface is burnished. Hard, very sparsely porous, greyish brown (10YR $5 / 2)$ and grey $(2.5 Y 5 / 1)$ fabric with frequent sand inclusions.

71. (No. 714): Body fragment; Kimistene, Acropolis, temple's terrace, illegally excavated area under the temple's podium, found in 2005. pl. 29/71.

Max. h $6.0 \mathrm{~cm}$., max. w $6.8 \mathrm{~cm}$., max. th 1.1 $\mathrm{cm}$.

Very dark grey (Gley $13 / \mathrm{N})$ slip on exterior; dark grey (2.5Y 4/1) unslipped surface on interior. Exterior surface is burnished. Average hardness; non-porous, fine, dark grey $(2.5 \mathrm{Y}$ 4/1) fabric with frequent tiny lime inclusions.

72. (No. 736): Body fragment; Kimistene, Acropolis, temple's terrace, illegally excavated area under the temple's podium, found in 2005. pl. 29/72.

Max. h $6.6 \mathrm{~cm}$., max. w $6.3 \mathrm{~cm}$., max. th 1.1 $\mathrm{cm}$.
Grey (2.5Y 5/1) slip on exterior; grey (10YR 5/1) unslipped surface on interior. Exterior surface is burnished. Average hardness; nonporous, brown (7.5YR 4/3) and very dark grey $(2.5 \mathrm{Y} 3 / 1)$ fabric with frequent micaceous and occasional lime inclusions.

73. (No. 713): Body fragment; Kimistene, Acropolis, temple's terrace, illegally excavated area under the temple's podium, found in 2005. pl. 29/73.

Max. h 8.5 cm., max. w 11.5 cm., max. th 0.9 $\mathrm{cm}$.

Very dark grey $(2.5 \mathrm{Y} 3 / 1)$ slip on exterior; very dark grey (10YR 3/1) unslipped surface on interior. Exterior surface is burnished. Average hardness; very sparsely porous, fine, dark grey (10YR 4/1) fabric with frequent sand, lime and large grit inclusions.

III. IRON AGE PAINTED WARE (pls. 2-3, nos. 74-98)

The Iron Age painted wares characterise Middle and Late Iron Age surface assemblages from surveyed sites to the south and southeast of Inner Paphlagonia, as at Gordion, Pazarl, Kaman Kalehöyük, Çadır Höyük, sites in Tokat and Sivas in Central Anatolia whereas looking northwards, the Late Iron Age painted pottery of İkiztepe near the Black Sea coast finds no clear parallels in the Paphlagonia survey material. Also in the surveys at Kastamonu no Iron Age painted ware were recorded. The small-scale excavations at Sinope brought to light a number of examples of local Anatolian Iron Age pottery (Akurgal, Budde 1956: 49, pl. 3), which, in some literature, has been wrongly named 'Phrygian pottery'. It is remarkable that no such pottery sherds have been found during the intensive surveys of the hinterland of Sinope (Summerer 2007, 30; Doonan 2004: 88). 
During the Iron Age brown-on-buff decoration is most common (Kealhofer et al. 2010, 84). Brown-on-buff styles include a range of decorative elements: hatched triangles, wavy lines, bands, concentric circles, geometric shapes, pendants and combinations of these (Kealhofer et al. 2010, 84).

In southwestern Paphlagonia this group has a heterogenous nature; their chronological and ethnic classification is very difficult. The sole distinctive characteristic is their painted (mostly geometric) decoration of two or three colours. 25 sherds were collected, 12 of which belong to open and 13 to closed forms. Open forms could be assigned to a bowl form.

Generally, closed forms have thick walls, and open forms have thin walls. Their paste differs from reddish yellow (5YR 6/6-6/8-7/6-7/8, 7.5YR 6/6-7/6), light brown (7.5YR 6/4), light red (2.5YR 6/6-6/8), light yellowish brown (10YR 6/4), brown (7.5YR 5/2), dark greyish brown (10YR 4/2), very pale brown (10YR 7/3), red (2.5YR 5/6), pink (5YR 7/4). Lime, sand, grog and micaceous inclusions are not visible on the surface. The paste is hard and of good quality with soapy slip in very pale brown (10YR 8.5/2-8/2-8/3- 8/4-7/3-7/4), pink (7.5YR 7/4-7/38/4), reddish yellow (5YR 7/8-7/6, 7.5YR 7/6), pale brown (10YR 6/3), white (10YR 8/1, 7.5YR $8 / 1)$, reddish brown (5YR 5/4), red (2.5YR 4/6) and strong brown $(7.5 \mathrm{YR} 5 / 8)$ colours at the interior or exterior surfaces. Non-slipped surfaces are pink (7.5YR 7/4), reddish yellow (5YR 6/67/6, 7.5YR 7/6-6/6), light brown (7.5YR 6/4) and light red $(2.5 \mathrm{YR} 6 / 6)$. Fine and non-porous clay is well-fired with lime, sand, grog and mica inclusions. Black, brown or a very dark grey painted decoration was applied to mostly light or unslipped surfaces. Colour of decoration changes from red (10R 4/6, 2.5YR 5/6), reddish brown (2.5YR 4/3-4/4-5/4, 5YR 4/3), dark reddish grey (2.5YR 3/1, 5YR 4/2), verk dark grey (5YR 3/1, 7.5YR 3/1, 10YR 3/1), weak red (10R 5/4-4/2-
4/3, 2.5YR 4/2), brown (7.5YR 4/2-4/3-4/4), reddish black (2.5YR 2.5/1), dark reddish brown (5YR 2.5/2), light red (2.5YR 6/6), yellowish red (5YR 4/6-5/6), strong brown (7.5YR 5/6), dark brown (7.5YR 3/2), dark greyish brown (10YR $4 / 2)$, to black (7.5YR 2.5/1).

On closed vessels decoration was applied to the belly and shoulder, in open vessels to their base. Painted decoration consists of mostly linear ornamentation; no figures or scenes were noted, as at Alişar IV. The most distinctive decorative pattern is the triangular hatch. Other decoration patterns are concentric circles, horizontal and vertical lines as well as combed triangles (for triangular hatches: Polat 1993, 43, fig. 14; 45, fig. 15; 51, fig. 18; Dönmez 2011, 127, fig. 29; and Bilgi 1999, 156, fig. 11.). In most cases decoration was made without any care. The painting style, colors, and decorative patterns are similar to those of Phrygian pottery. In the Museum of Çankırı there are some vessels with similar forms and decoration.

10 fragments were found on the southern slopes of the Acropolis at Kimistene, 7 from Kepez 4 from the Cistern, 1 from southeastern slope, 1 from western slope, and at the temple's terrace on the Acropolis of Kimistene. It is noteworthy that no painted sherds were found in the temple's temenos at Kimistene. Similar examples were found in the British survey (Matthews 2009, 153, fig. 5.2, no. 5, site PS 156; and 166, fig. 5.16, nos. 6-7, site PS 170). They resemble the finds from Kimistene in terms of outer slip, surface treatment, paste color and decorative patterns.

A large number of ceramic imports would not be expected in Paphlagonia during the Iron Age; this highlights the importance of reproduction and emulation by local Iron Age producers.

Late Iron Age; i.e. 6th-4th cent. B.C.

OPEN FORMS (pls. 2-3, nos. 74-85) / Rim Fragments of a Bowl Form (pls. 2-3, nos. 74-80) 
It consists of rim fragments of straight walled, incurved and slightly outcurved bowls.

74. (No. 488): Rim fragment; Kimistene, Acropolis, southern slope, just below the summit, up to Deresemail creek, found in 2005. pl. 2/74 and pl. 30/74.

Max. h $2.1 \mathrm{~cm}$., max. w $2.4 \mathrm{~cm}$., max. th 0.4 $\mathrm{cm}$.

Very pale brown (10YR 8/2) slip on exterior; reddish yellow (5YR 7/6) unslipped surface on interior. Decoration: Red (2.5YR 5/6) band on exterior rim; its below part bounded with very dark grey (10YR 3/1) thin horizontal band. Traces of same paint at elsewhere on the exterior. Hard, thin paste, very sparsely porous; fine, reddish yellow (5YR 7/8) fabric with no visible inclusions.

75. (No. 487): Rim fragment; Kimistene, Acropolis, southern slope, just below the summit, up to Deresemail creek, found in 2005. pl. 2/75 and pl. 30/75.

Max. h $2.6 \mathrm{~cm}$., max. w $2.6 \mathrm{~cm}$., max. th 0.3 $\mathrm{cm}$.

Very pale brown (10YR 8/2) slip on exterior; reddish yellow (5YR 7/8) slip on interior. Decoration: Red (2.5YR 5/6) band on exterior rim. It is bounded with very dark grey (2.5YR $3 / 1$ ) thin horizontal band on exterior. Immediately below are two semicircles; between them straight and wavy lines. Decoration painted with red (2.5YR 5/6) and dark reddish grey $(2.5 \mathrm{YR} 3 / 1)$. Hard, thin paste, very sparsely porous, fine, reddish yellow (5YR 6/8) fabric with no visible inclusions.

Parallel: Özsait/Özsait 1996, 365, 381, pl. I, no, 18, 21 (from Şeref Höyük).

76. (No. 1084): Rim fragment; Kepez, found in 2005. pl. 2/76 and pl. 30/76.

Max. h $3.1 \mathrm{~cm}$., max. w $1.6 \mathrm{~cm}$., max. th 0.4 $\mathrm{cm}$.
Very pale brown (10YR 8/4) slip on exterior. Interior surface slipped very pale brown (10YR 7/4). Decoration: Weak red (10R 5/4) band on exterior rim, its below part bounded with very dark grey (7.5YR 3/1), thin horizontal band on exterior. Immediately below two weak red (10R 5/4) circles are side by side. Hard, thin paste, non-porous, fine, reddish yellow (7.5YR 6/6) fabric with some lime inclusions.

77. (No. 1098): Rim of a bowl; Kepez, found in 2005. pl. 2/77 and pl. 30/77.

Max. h 2.7 cm., max. w 2.5 cm., max. th 0.5 $\mathrm{cm}$.

Very pale brown (10YR 8.5/2) slip on exterior; very pale brown (10YR 7/3) slip on interior. Reddish brown (5YR 4/3) decoration on exterior rim. Vertical and horizontal lines in dark reddish brown (5YR 2.5/2) as decoration patterns. Average hardness; thin paste, very sparsely porous, dark greyish brown (10YR 4/2) fabric with some tiny lime inclusions.

78. (No. 1089): Rim fragment of a bowl; Kepez, found 2005. pl. 2/78 and pl. 30/78.

Max. h $2.6 \mathrm{~cm}$., d of rim $14.4 \mathrm{~cm}$., max. w 3.1 cm., max. th $0.3 \mathrm{~cm}$.

Very pale brown (10YR 7/4) slip on exterior; very pale brown (10YR 7/3) slip on interior. Red (2.5YR 5/6) band on exterior rim; other part is very dark grey (7.5YR 3/1). A thin horizontal band and immediately below is the upper part of the two inner circles, side by side. Hard, thin paste, non-porous, fine, very pale brown (10YR 7/3) fabric with no visible inclusions.

79. (No. 1097): Rim fragment; Kepez, found in 2005. pl. 3/79 and pl. 30/79.

Max. h $2.3 \mathrm{~cm}$., d of rim $16.4 \mathrm{~cm}$., max. w 2.8 cm., max. th $0.5 \mathrm{~cm}$.

Very pale brown (10YR 8.5/2) slip on exterior; 
red (2.5YR 4/6) slip on interior. Red (2.5YR $5 / 6)$ band on exterior rim. Horizontal and vertical lines in form of a square below the rim. Dark reddish grey (2.5YR 3/1) decoration on exterior. Average hardness; thin paste, nonporous, fine, reddish yellow (7.5YR 6/6) fabric with frequent tiny lime inclusions.

80. (No. 1171): Rim fragment of an open vessel form; a surface find from the Cistern in Kepez, found in 2005. pl. 3/80 and pl. 30/80. Max. h 2.4 cm., d of rim 24.2 cm., max. w 5.4 $\mathrm{cm}$, max. th $0.4 \mathrm{~cm}$.

Very pale brown (10YR 8/2) slip on exterior; red $(2.5 \mathrm{YR} 5 / 6)$ slip on interior. Reddish brown (2.5YR 5/4) band on exterior rim. Applique decoration on exterior rim. At lower part two red (2.5YR 5/6) lines. Average hardness; thin paste, very sparsely porous, fine, light yellowish brown (10YR 6/4) fabric with infrequent lime inclusions.

Parallel: Dönmez 2010b, 48, draw. 18, fig. 76 (from Oluz Höyük, dated to the Late Iron Age).

\section{Body Fragments of Open Forms (pl. 3,} no. 85)

Their typologies are difficult to judge.

81. (No. 654): Body fragment; Kimistene, Acropolis, southern slope, just below the summit, up to Deresemail creek, found in 2005. pl. 30/81.

Max. h 1.8 cm., max. w $1.7 \mathrm{~cm}$., max. th 0.4 $\mathrm{cm}$.

Pink (7.5YR 7/4) slip on exterior; reddish yellow (5YR 7/6) slip on interior. Reddish black (2.5YR 2.5/1) and light red (2.5YR 6/6) lines may belong to circle. Hard, non-porous, thin paste, fine, reddish yellow (5YR 6/6) fabric with no visible inclusions.
82. (No. 1065): Body fragment; Kepez, found in 2005. pl. 30/82.

Max. h $2.3 \mathrm{~cm}$., max. w $1.9 \mathrm{~cm}$., max. th 0.5 $\mathrm{cm}$.

Very pale brown (10YR 8.5/2) slip on exterior; reddish brown (5YR 5/4) slip on interior. Reddish brown (5YR 4/3) and yellowish red (5YR 4/6) decoration on exterior surface. Average hardness; thin paste, very sparsely porous, fine, light brown (7.5YR 6/4) fabric with no visible inclusions.

83. (No. 803): Body fragment; Kimistene, summit of the Acropolis, western slope, found in 2005. pl. 30/83.

Max. h $2.0 \mathrm{~cm}$., max. w $2.5 \mathrm{~cm}$., max. th 0.5 $\mathrm{cm}$.

White (7.5YR 8/1) slip on exterior; reddish yellow (7.5YR 7/6) slip on interior. Thin and straight line is with reddish brown $(2.5 \mathrm{YR}$ $4 / 4$ ) on interior surface. Hard, thin paste, very sparsely porous, fine, reddish yellow (7.5YR $7 / 6$ ) fabric with no visible inclusions.

84. (No. 1033): Body fragment; Kepez, found in 2005. pl. 30/84.

Max. h $4.0 \mathrm{~cm}$., max. w $2.6 \mathrm{~cm}$., max. th 0.6 $\mathrm{cm}$.

Very pale brown (10YR 8/2) slip on exterior; pink (7.5YR 7/4) slip on interior. Brown (7.5YR 4/4) and strong brown (7.5YR 5/6) ornaments on exterior surface. Hard, thin paste, very sparsely porous, fine, reddish yellow (5YR 6/6) fabric with some tiny lime inclusions.

85. (No. 920): Body fragment of an open form; Kimistene, on southeastern slopes to the Cistern, surface find, found in 2005. pl. 3/85 and pl. 30/85.

Max. h 1.4 cm., max. w $3.9 \mathrm{~cm}$., max. th 0.9 $\mathrm{cm}$.

Reddish yellow (5YR 6/6) unslipped surface 
on exterior; pink (7.5YR 7/4) slip on interior. Black (7.5YR 2.5/1) geometric ornaments on exterior surface. On interior face triangular with horizontal and vertical ornamentations. Hard, non-porous, thin paste, fired to reddish yellow (5YR 6/6) and light red (2.5YR 6/8) fabric with rare tiny lime inclusions.

CLOSED FORMS (pl. 3, nos. 86-98) / A Rim Fragment (pl. 3, no. 86)

A short necked closed form.

86. (No. 1446): Rim fragment; Kimistene, Acropolis, temple's terrace, found in 2005. pl. 3/86 and $\mathrm{pl.} \mathrm{30/86.}$

Max. h $4.5 \mathrm{~cm}$., d of rim $23.6 \mathrm{~cm}$., max. w 6.2 cm., max. th $0.8 \mathrm{~cm}$.

Pink (7.5YR 8/4) slip on exterior; pink (7.5YR 7/4) unslipped surface on interior. Three bands and geometric ornaments in very dark grey (7.5YR 3/1) and yellowish red (5YR $5 / 6$ ) paint on exterior. Hard, non-porous, thin paste, fine, reddish yellow (7.5YR 7/6) fabric with some tiny lime inclusions.

Parallel: Dönmez 2010a, 306, fig. 37 (from Oluz Höyük, dated to the Late Iron Age).

Handle Fragments of Closed Forms (pl. 3 , nos. 87-88)

Vertical handles.

87. (No. 914): Handle fragment; Kimistene, Acropolis, on the southeastern slope, in a rugged area to Sarpin Çay, surface find, found in 2005. pl. 3/87 and pl. 30/87.

Max. h $3.2 \mathrm{~cm}$., max. w $4.4 \mathrm{~cm}$., max. th 1.8 $\mathrm{cm}$.

Very pale brown (10YR 8/2) eroded slip all of surface. Hard, thin paste, very sparsely porous, fine, reddish yellow (5YR 7/6) fabric with rare sand and lime inclusions.
88. (No. 1323): Handle fragment; Kimistene, summit of the Acropolis, southern slope, found in 2005. pl. 3/88 and pl. 30/88.

Max. h $5.2 \mathrm{~cm}$., max. w $5.4 \mathrm{~cm}$., max. th 0.7 $\mathrm{cm}$.

Th. of hand $1.7 \mathrm{~cm}$., w of hand $2.9 \mathrm{~cm}$.

Very pale brown (10YR 8.5/2) slip on exterior; white (10YR 8/1) slip on interior. Light brown (7.5YR 6/4) unslipped surface on interior handle. Dark reddish grey (5YR 4/2) and brown $(7.5 \mathrm{YR} 4 / 2)$ geometric ornaments on exterior surface. Hard, sparsely porous, thin paste, fired to light brown (7.5YR 6/4) and brown $(7.5 \mathrm{YR} 5 / 2)$ fabric with some micaceous, sand and rare lime inclusions.

Parallels: Dönmez 2010a, 290, 306, fig. 37 (from Oluz Höyük, Level 3, 5th-3rd cent. B.C.); Kealhofer et al. 2010, 83, Group 5, no. e (G5 Ç 3412).

Body Fragments of Closed Forms (pl. 3, nos. 89-98)

89. (No. 843): Body fragment; Kimistene, Cistern, eastern slope, found in 2005. pl. 3/89 and $\mathbf{p l . 3 0 / 8 9}$.

Max. h $1.6 \mathrm{~cm}$., max. w $1.5 \mathrm{~cm}$., max. th 0.7 $\mathrm{cm}$.

Pale brown (10YR 6/3) slip on exterior and interior. Reddish brown (2.5YR 4/3) geometric ornaments on exterior surface. Hard, nonporous, thin paste, fired to light brown (7.5YR 6/4) and light red (2.5YR 6/6) fabric with rare sand inclusions.

Parallels: Dönmez 2010a, 306, fig. 37 (from Oluz Höyük, dated to the Late Iron Age).

90. (No. 1330): Body fragment; Kimistene, summit of the Acropolis, southern slope, found in 2005. pl. 30/90.

Max. h $2.1 \mathrm{~cm}$., max. w $1.6 \mathrm{~cm}$., max. th 0.4 $\mathrm{cm}$.

Light red (2.5YR 6/6) unslipped surface on 
exterior and interior. Horizontal and vertical lines on exterior surface. They are painted in weak red (10R 4/3). Hard, thin paste, non-porous, fine, light red (2.5YR 6/6) fabric with no visible inclusions.

91. (No. 1437): Body fragment; Kimistene, Acropolis, southern slope, just below the summit, up to Deresemail creek, found in 2005. pl. 3/91 and pl. 30/91.

Max. h 1.9 cm., max. w 3.1 cm., max. th 0.6 cm.

Pink (7.5YR 7/3) slip on exterior; reddish yellow (5YR 6/6) unslipped surface on interior. Weak red (2.5YR 4/2) geometric decoration on exterior surface. Hard, thin paste, non-porous, fine, light red (2.5YR 6/6) fabric with rare grog and some sand inclusions.

92. (No. 550): Body fragment of a closed form; Kimistene, Acropolis, southern slope, just below the summit, up to Deresemail creek, found in 2005. pl. 3/92 and pl. 30/92.

Max. h 2.7 cm., max. w $3.3 \mathrm{~cm}$., max. th 0.6 cm.

Strong brown (7.5YR 5/8) slip on exterior; reddish yellow (7.5YR 6/6) unslipped surface on interior. Brown (7.5YR 4/3) geometric ornaments on exterior slip. Hard, thin paste, very sparsely porous, fine, red (2.5YR 5/6) fabric with frequent tiny lime and some sand inclusions.

93. (No. 715): Body fragment; Kimistene, Acropolis, southern slope, just below the summit, up to Deresemail creek, found in 2005. pl. 3/93 and pl. 30/93.

Max. h $2.3 \mathrm{~cm}$., max. w $4.0 \mathrm{~cm}$., max. th 0.6 $\mathrm{cm}$.

Very pale brown (10YR 8.5/2) cracked slip on exterior; very pale brown (10YR 8/2) slip on interior. Dark reddish grey (2.5YR 3/1) vertical lines on upper exterior. This decora- tion bounded with two weak red (10R 4/2) and dark reddish grey (2.5YR 3/1) horizontal bands on exterior. At the lower part has brown (7.5YR 4/3) surface on exterior. Hard, thin paste, non-porous, fine, light yellowish brown (10YR 6/4) fabric with no visible inclusions.

94. (No. 886): Body fragment; Kimistene, Acropolis, southern slope, underground cave, surface find, found in 2005. pl. 30/94.

Max. h 4.4 cm., max. w $4.6 \mathrm{~cm}$., max. th 0.8 $\mathrm{cm}$.

Pink (7.5YR 8/4) slip on exterior; light brown (7.5YR 6/3) unslipped surface on interior. Hard, non-porous, thin paste, fired to light brown (7.5YR 6/4) fabric with occasional sand inclusions.

95. (No. 440): Body fragment; Kimistene, Acropolis, slope, found in 2005. pl. 30/95.

Max. h $4.2 \mathrm{~cm}$., max. w $5.6 \mathrm{~cm}$., max. th 1.1 $\mathrm{cm}$.

Pink (7.5YR 7/4) unslipped surface on exterior and interior. Some lines as decoration pattern. It is with very dark grey (5YR 3/1). Hard, thin paste, non-porous, fine, pink (5YR 7/4) fabric with rare tiny lime and sand inclusions.

96. (No. 1438): Body fragment; Kimistene, Acropolis, southern slope, just below the summit, up to Deresemail creek, found in 2005.

pl. 3/96 and pl. 30/96.

Max. h $5.0 \mathrm{~cm}$., max. w $4.0 \mathrm{~cm}$., max. th 0.7 $\mathrm{cm}$.

Very pale brown (10YR 8/3) slip on exterior; reddish yellow (7.5YR 7/6) unslipped surface on interior. Red (2.5YR 5/6) horizontal band and dark brown (7.5YR 3/2) geometric ornaments on exterior slip. Hard, thin paste, very sparsely porous, fine, reddish yellow (7.5YR 6/6) fabric with frequent tiny lime and rare sand inclusions. 
97. (No. 974): Body fragment; Kimistene, the surface find of the Cistern, eastern slope, found in 2005. pl. 30/97.

Max. h $5.2 \mathrm{~cm}$., max. w $6.3 \mathrm{~cm}$., max. th 1.3 $\mathrm{cm}$.

Very pale brown (10YR 8/3) slip on exterior; pink (7.5YR 7/4) unslipped surface on interior. Dark greyish brown (10YR 4/2) line on exterior. Hard, thin paste, very sparsely porous, fine, reddish yellow (5YR 6/6) fabric with some tiny lime and rare sand inclusions.

98. (No. 1284): Body fragment of a closed form; Kimistene, on southeastern slopes to the Cistern, found in 2005. pl. $3 / 98$ and pl. $30 / 98$.

Max. h $6.2 \mathrm{~cm}$., max. w $6.6 \mathrm{~cm}$., max. th 1.0 $\mathrm{cm}$.

Pink (7.5YR 7/4) unslipped surface on exterior; pink (7.5YR 7/4) unslipped surface on interior. Very dark grey (5YR 3/1) geometric ornaments on exterior surface which is bounded with very dark grey (5YR 3/1) horizontal band. All of the surface of the lower part has a red (10R 4/6) paint. Hard, thin paste, nonporous, fine, reddish yellow (5YR 6/6) fabric with rare micaceous inclusions.

Parallels: Dönmez 2005b, 68, pl. 4/10=fig. 19 (from Köyiçi in Samsun); <http://oi.uchicago. edu/pdf/ar/01-10/08-09/08-09_AnnualReport.pdf>, fig. 20 (from Çadır Höyük; Middle Iron Ages).

IV. IRON AGE COARSE WARE (pl. 4, nos. 99-104)

This group consists of very small sherds, mostly (25 fragments) from an illegally excavated area under the temple's podium on the Acropolis of Kimistene. They belong to thick-walled forms with an extremly coarse fabric. The assemblage includes jugs, jars and cooking pots. 30 sherds in total were collected, 5 of which belong to open and 25 to closed forms. The clay is reddish brown (5YR 4/3-5/4), reddish yellow (5YR 6/6-7/6, 7.5YR 7/6), pink (5YR 7/4, 7.5YR 7/4), brown (7.5YR 4/3-4/4-5/4-5/3), very pale brown (10YR 7/3-7/4), light brown (7.5YR 6/4), light brownish grey (10YR 6/2), yellowish red (5YR 5/6-4/6), red (2.5YR 5/6), light red (2.5YR 6/8). Most of them are badly fired; therefore their surfaces have grey (5YR 4/1, 7.5YR 4/1-3/1, 10YR 5/1-4/1, 2.5Y $3 / 1$ ) or brown (10YR 5/2,6/3, 7/4, 6/2) variations. Major inclusions are sand, micaceous, lime and plants. The clay is medium or very hard. Surfaces are not very porous.

Their slip is reddish yellow (5YR 6/6-6/8, 7.5YR 7/6-6/6), pale brown (10YR 6/3), brown (7.5YR 5/2-5/3-5/4), very pale brown (10YR 7/3), pink (7.5YR 7/4-8/3), light grey (10YR 7/2), light reddish brown (5YR 6/4) and grey (10YR 6/1). All of them are wheel-made, except for no. 111. Most of the surfaces have traces of burning.

Rim Fragment of an Open Form (pl. 4, no. 99)

99. (No. 742): Rim fragment; Kimistene, Acropolis, temple's terrace, illegally excavated area under the temple's podium, found in 2005. pl. 4/99 and pl. 30/99.

Max. h $2.5 \mathrm{~cm}$., max. w $4.0 \mathrm{~cm}$., max. th 1.0 $\mathrm{cm}$.

Brown (7.5YR 5/3) slip on exterior rim; brown (7.5YR 5/2) slip on interior. Soft, nonporous, reddish brown (5YR 5/4) and dark grey (5YR 4/1) fabric with some sand and micaceous inclusions. Traces of burning on the exterior surface.

Body Fragments of Open Forms (pls. 3031, nos. 100-103)

100. (No. 963): Body fragment; Kimistene, Acropolis, found in 2005. pl. 30/100. 
Max. h 1.7 cm., max. w $1.6 \mathrm{~cm}$, max. th 1.0 $\mathrm{cm}$.

Light grey (10YR 7/2) unslipped surface on exterior; pale brown (10YR 6/3) slip on interior. Average hardness; very sparsely porous, fine, light brownish grey (10YR 6/2) fabric with frequent tiny lime and medium grit inclusions.

101. (No. 766): Body fragment; Kimistene, Acropolis, temple's terrace, illegally excavated area under the temple's podium, found in 2005. pl. 30/101.

Max. h $2.2 \mathrm{~cm}$., max. w $2.1 \mathrm{~cm}$., max. th 1.1 $\mathrm{cm}$.

Grey (10YR 6/1) slip on exterior; brown (7.5YR 5/2) slip on interior. Average hardness; non-porous, reddish brown (5YR 4/3) and grey (10YR 5/1) fabric with frequent tiny lime and sand inclusions. Traces of burning on the exterior surface.

102. (No. 775): Body fragment; Kimistene, Acropolis, temple's terrace, illegally excavated area under the temple's podium, found in 2005. pl. 30/102.

Max. h $3.3 \mathrm{~cm}$., max. w $3.2 \mathrm{~cm}$., max. th 1.1 $\mathrm{cm}$.

Reddish yellow (5YR 7/6) unslipped surface on exterior; reddish yellow (7.5YR 7/6) slip on interior. Average hardness; sparsely porous, very pale brown (10YR 7/4) and reddish yellow (5YR 7/6) fabric with frequent some sand large grit inclusions.

103. (No. 757): Body fragment; Kimistene, Acropolis, temple's terrace, illegally excavated area under the temple's podium, found in 2005. pl. 31/103.

Max. h $3.2 \mathrm{~cm}$., max. w $3.4 \mathrm{~cm}$., max. th 1.0 $\mathrm{cm}$.

Light grey (10YR 7/2) slip on exterior; pale brown (10YR 6/3) slip on interior. Average hardness; non-porous, dark grey (10YR 4/1) fabric with occasional lime and rare micaceous inclusions. Traces of burning on the exterior surface.

CLOSED FORMS (pl. 4, no. 104) / Rim Fragments (pl. 4, no. 104)

No. 104 is a outcurved rim form.

104. (No. 844): Rim fragment; Kimistene, Cistern, eastern slope, found in 2005. pl. 4/104 and pl. 31/104.

Max. h $1.6 \mathrm{~cm}$., d of rim $9.6 \mathrm{~cm}$. , max. w 3.1 cm., max. th $0.7 \mathrm{~cm}$.

Brown (7.5YR 5/4) unslipped surface on exterior and interior. Soft, non-porous, fired to brown (7.5YR 4/4) and black (Gley $12.5 / \mathrm{N}$ ) fabric with frequent lime and small grit inclusions.

105. (No. 740): Rim fragment; Kimistene, Acropolis, temple's terrace, illegally excavated area under the temple's podium, found in 2005. pl. 31/105.

Max. h $2.8 \mathrm{~cm}$., max. w $3.9 \mathrm{~cm}$., max. th 1.0 $\mathrm{cm}$.

Pink (7.5YR 7/4) unslipped surface on exterior and interior. Hard, non-porous, fine, reddish yellow (7.5YR 7/6) fabric with frequent large grit inclusions.

Body Fragments of Closed Forms (pls. 31-32, nos. 106-128)

There are burning traces on interior surface at nos. 106 and 110-111, at exterior surface at nos. $107-108,126$ and on whole surface at no. 128. On nos. 117, 120-121 and 128 there are strong wheelmarks in the interior surface. On no. 122 exterior surface is extremly micaceous. 
106. (No. 776): Body fragment; Kimistene, Acropolis, temple's terrace, illegally excavated area under the temple's podium, found in 2005. pl. 31/106.

Max. h $1.8 \mathrm{~cm}$., max. w $1.9 \mathrm{~cm}$., max. th 0.8 $\mathrm{cm}$.

Light brown (7.5YR 6/3) unslipped surface on exterior; brown (7.5YR 5/4) unslipped surface on interior. Average hardness; very sparsely porous, fine, reddish brown (5YR 4/3) fabric with frequent sand, medium grit and rare micaceous inclusions.

107. (No. 763): Body fragment; Kimistene, Acropolis, temple's terrace, illegally excavated area under the temple's podium, found in 2005. pl. 31/107.

Max. h 2.8 cm., max. w 2.9 cm., max. th 0.9 $\mathrm{cm}$.

Greyish brown (10YR 5/2) unslipped surface on exterior; brown (7.5YR 5/2) unslipped surface on interior. Average hardness; non-porous, fired to brown (7.5YR 4/3) fabric with frequent tiny lime, some medium grit and micaceous inclusions.

108. (No. 772): Body fragment; Kimistene, Acropolis, temple's terrace, illegally excavated area under the temple's podium, found in 2005. pl. 31/108.

Max. h $2.6 \mathrm{~cm}$., max. w $3.5 \mathrm{~cm}$., max. th 0.8 $\mathrm{cm}$.

Light brownish grey (2.5Y 6/2) unslipped surface on exterior; pale brown (10YR 6/3) unslipped surface on interior. Average hardness; very sparsely porous, fired to brown (7.5YR $5 / 4$ ) and greyish brown (10YR 5/2) fabric with frequent medium grit inclusions.

109. (No. 792): Body fragment; Kimistene, Acropolis, temple's terrace, illegally excavated area under the temple's podium, found in 2005. pl. 31/109.
Max. h $2.6 \mathrm{~cm}$., max. w $4.6 \mathrm{~cm}$., max. th 1.1 $\mathrm{cm}$.

Reddish yellow (5YR 6/6) slip on exterior; pink (7.5YR 7/4) unslipped surface on interior. Hard, non-porous, fine, pink (7.5YR 7/4) fabric with frequent sand and rare micaceous inclusions.

110. (No. 768): Body fragment; Kimistene, Acropolis, temple's terrace, illegally excavated area under the temple's podium, found in 2005. pl. 31/110.

Max. h $3.1 \mathrm{~cm}$., max. w $3.4 \mathrm{~cm}$., max. th 1.0 $\mathrm{cm}$.

Brown (7.5YR 5/4) slip on exterior; greyish brown (10YR 5/2) unslipped surface on interior. Average hardness; very sparsely porous, fired to yellowish red (5YR 4/6) and greyish brown $(10 \mathrm{YR} 5 / 2)$ fabric with frequent tiny lime, some medium grit inclusions.

111. (No. 1126): Body fragment; Kimistene, Acropolis, found in 2005. pl. 31/111.

Max. h $2.3 \mathrm{~cm}$., max. w $3.4 \mathrm{~cm}$., max. th 0.6 $\mathrm{cm}$.

Pale brown (10YR 6/3) slip on exterior; light brownish grey (10YR 6/2) unslipped surface on interior. Exterior surface is burnished. Hard, non-porous, fired to light brownish grey (10YR 6/2) and very dark grey (2.5Y 3/1) fabric with some tiny lime and large grit inclusions.

112. (No. 799): Body fragment; Kimistene, Acropolis, temple's terrace, illegally excavated area under the temple's podium, found in 2005. pl. 31/112.

Max. h $3.6 \mathrm{~cm}$., max. w $3.8 \mathrm{~cm}$., max. th 1.2 $\mathrm{cm}$.

Very pale brown (10YR 7/3) slip on exterior; light reddish brown (5YR 6/4) slip on interior. Hard, non-porous, fine, pink (7.5YR 7/4) fabric with frequent medium grit inclusions. 
113. (No. 784): Body fragment; Kimistene, Acropolis, temple's terrace, illegally excavated area under the temple's podium, found in 2005. pl. 31/113.

Max. h 3.8 cm., max. w $4.0 \mathrm{~cm}$., max. th 1.2 $\mathrm{cm}$.

Light brown (7.5YR 6/4) unslipped surface exterior; light grey (10YR 7/2) unslipped surface on interior. Average hardness; nonporous, fired to light brown (7.5YR 6/4) and pale brown (10YR 6/3) fabric with some sand and lime inclusions.

114. (No. 795): Body fragment; Kimistene, Acropolis, temple's terrace, illegally excavated area under the temple's podium, found in 2005. pl. 31/114.

Max. h 4.0 cm., max. w $5.0 \mathrm{~cm}$., max. th 1.4 $\mathrm{cm}$.

Very pale brown (10YR 7/3) unslipped surface on exterior, pink (7.5YR 7/3) unslipped surface on interior, hard, very sparsely porous, fired to brown (7.5YR 5/3) fabric with some tiny lime, small grit and sand inclusions.

115. (No. 481): Body fragment; Kimistene, Acropolis, southern slope, just below the summit, up to Deresemail creek, found in 2005. pl. 31/115.

Max. h 4.1 cm., max. w 5.1 cm., max. th 1.4 $\mathrm{cm}$.

Light grey (10YR 7/2) slip on exterior; very pale brown (10YR 7/3) unslipped surface on interior. Exterior surface is burnished. Hard, very sparsely porous, fired to greyish brown (10YR 5/2) and reddish brown (5YR 5/4) fabric with some lime and micaceous inclusions.

116. (No. 767): Body fragment; Kimistene, Acropolis, temple's terrace, illegally excavated area under the temple's podium, found in 2005. pl. 31/116.

Max. h 4.6 cm., max. w 4.8 cm., max. th 1.4 $\mathrm{cm}$.

Reddish yellow (7.5YR 7/6) slip on exterior; light red (2.5YR 6/6) on exterior slip. Very pale brown (10YR 7/4) unslipped surface on interior. Hard, non-porous, fired to pink (7.5YR 7/4) and very pale brown (10YR 7/4) fabric with some sand and large grit inclusions.

117. (No. 758): Body fragment; Kimistene, Acropolis, temple's terrace, illegally excavated area under the temple's podium, found in 2005. pl. 31/117.

Max. h $5.1 \mathrm{~cm}$., max. w $3.3 \mathrm{~cm}$., max. th 1.9 $\mathrm{cm}$.

Pink (7.5YR 7/4) unslipped surface exterior and interior. Hard, non-porous, fine, reddish yellow (5YR 7/6) fabric with frequent sand, lime and large grit inclusions.

118. (No. 686): Body fragment; Kimistene, Cistern, eastern slope, found in 2005. pl. 31/118.

Max. h $4.7 \mathrm{~cm}$., max. w $5.3 \mathrm{~cm}$., max. th 1.3 $\mathrm{cm}$.

Reddish yellow (5YR 6/8) slip on exterior; reddish yellow (5YR 6/8) unslipped surface on interior. Hard, non-porous, fine, light red (2.5YR 6/8) fabric with some medium grit and tiny lime inclusions.

119. (No. 783): Body fragment; Kimistene, Acropolis, temple's terrace, illegally excavated area under the temple's podium,found in 2005. pl. 31/119.

Max. h 4.4 cm., max. w 6.9 cm., max. th 0.9 $\mathrm{cm}$.

Pink (5YR 7/4) unslipped surface on exterior; light reddish brown (5YR 6/4) unslipped surface on interior. Hard, very sparsely porous, fine, reddish brown (5YR 5/4) fabric with frequent medium grit and lime inclusions. 
120. (No. 790): Body fragment; Kimistene, Acropolis, temple's terrace, illegally excavated area under the temple's podium, found in 2005. pl. 31/120.

Max. h 4.9 cm., max. w $5.1 \mathrm{~cm}$., max. th 1.7 $\mathrm{cm}$.

Reddish yellow (7.5YR 6/6) slip on exterior; very pale brown (10YR 7/4) unslipped surface on interior. Hard, very sparsely porous, fired to light brownish grey (10YR 6/2) and very pale brown (10YR 7/4) fabric with frequent large grit inclusions.

121. (No. 794): Body fragment; Kimistene, Acropolis, temple's terrace, illegally excavated area under the temple's podium, found in 2005. pl. 31/121.

Max. h 4.8 cm., max. w 5.5 cm., max. th 1.4 $\mathrm{cm}$.

Pink (7.5YR 8/3) slip on exterior; pink (7.5YR 7/4) unslipped surface on interior. Hard, nonporous, fine, reddish yellow (5YR 6/6) fabric with some medium grit and sand inclusions.

122. (No. 749): Body fragment; Kimistene, Acropolis, temple's terrace, illegally excavated area under the temple's podium, found in 2005. pl. 31/122.

Max. h 5.8 cm., max. w 4.2 cm., max. th 1.1 $\mathrm{cm}$.

Very pale brown (10YR 7/4) unslipped surface on exterior and interior. Hard, very sparsely porous, fine, very pale brown (10YR 7/3) fabric with frequent lime, micaceous and some medium grit inclusions.

123. (No. 786): Body fragment; Kimistene, Acropolis, temple's terrace, illegally excavated area under the temple's podium, found in 2005. pl. 31/123.

Max. h 5.9 cm., max. w $5.8 \mathrm{~cm}$., $\max$. th 1.2 $\mathrm{cm}$.

Pink (5YR 7/4) unslipped surface on exterior; reddish yellow (5YR 6/6) unslipped surface on interior. Hard, very sparsely porous, fine, reddish yellow (5YR 6/6) fabric with some large grit and lime inclusions.

124. (No. 1290): Body fragment; Kimistene, Acropolis, temple slope, illegally excavated pit, found in 2005. pl. 31/124.

Max. h $6.0 \mathrm{~cm}$., max. w $4.2 \mathrm{~cm}$., max. th 1.1 $\mathrm{cm}$.

Pink (7.5YR 7/4) slip on exterior, light grey (10YR 7/2) unslipped surface on interior. A red $(2.5 \mathrm{YR} 5 / 6)$ band on exterior. Exterior surface is burnished. Soft, thin paste, nonporous, pink (5YR 7/4) and very pale brown (10YR 7/4) fabric with infrequent medium grit inclusions.

125. (No. 797): Body fragment; Kimistene, Acropolis, temple's terrace, illegally excavated area under the temple's podium, found in 2005. pl. 32/125.

Max. h $6.0 \mathrm{~cm}$., max. w $6.1 \mathrm{~cm}$., max. th 1.3 $\mathrm{cm}$.

Very pale brown (10YR 7/3) slip on exterior; reddish yellow (5YR 7/6) unslipped surface on interior. Hard, sparsely porous, red (2.5YR $5 / 6$ ) fabric with frequent lime, sand, large grit inclusions.

126. (No. 789): Body fragment; Kimistene, Acropolis, temple's terrace, illegally excavated area under the temple's podium, found in 2005. pl. 32/126.

Max. h 6.4 cm., max. w 6.2 cm., max. th 1.6 $\mathrm{cm}$.

Pink (5YR 7/4) unslipped surface on exterior; pink (7.5YR 7/4) unslipped surface interior. Hard, very sparsely porous, reddish brown (5YR 5/4) and dark grey (7.5YR 4/1) fabric with frequent sand, tiny lime and some large grit, plant inclusions. 
127. (No. 788): Body fragment; Kimistene, Acropolis, temple's terrace, illegally excavated area under the temple's podium, in 2005. pl. 32/127.

Max. h $6.6 \mathrm{~cm}$., max. w $9.8 \mathrm{~cm}$., max. th 2.1 $\mathrm{cm}$.

Light brown (7.5YR 6/4) unslipped surface on exterior; very pale brown (10YR 7/3) unslipped surface interior. Hard, very sparsely porous, light brown (7.5YR 6/4) fabric with frequent large grit and sand inclusions.

128. (No. 798): Body fragment; Kimistene, Acropolis, temple's terrace, illegally excavated area under the temple's podium, found in 2005. pl. 32/128.

Max. h 9.8 cm., max. w $9.8 \mathrm{~cm}$., max. th 1.2 $\mathrm{cm}$.

Light brown (7.5YR 6/4) unslipped surface on exterior and interior. Average hardness; non-porous, fired to yellowish red (5YR 5/6) and very dark grey (7.5YR 3/1) fabric with frequent sand and large grit inclusions.

\section{PONTIC SKYPHOS FRAGMENT} (pl. 4, no. 129)

During the 2005 survey at Kimistene one single fragment of a skyphos was recovered. Its shape, the quality of its slip, and its decoration resemble black-glazed vessels found in the northern Black Sea region. Many of the Atticizing wares found in Early Hellenistic contexts at Gordion were also imported from the Black Sea rather than from Aegean production centers (Stewart 2010, 84). The fabric is clean and reddish. It has a stamped decoration on the interior surface. It should be dated to the early 4th century B.C. A similar black-glazed fragment of 4th-3rd cent. B.C. was found at Oluz Höyük (Dönmez 2010b, 63, fig. 103.).
129. (No. 888): Body fragment; Kimistene, Acropolis, southern slope, underground cave, surface find, found in 2005. pl. 4/129 and pl. 32/129.

Max. h 2.4 cm., max. w $4.3 \mathrm{~cm}$., max. th 0.8 $\mathrm{cm}$.

Black (Gley $12.5 / \mathrm{N}$ ) slip is worn off. Hard, non-porous, thin paste, fired to red $(2.5 \mathrm{YR}$ $5 / 8$ ) fabric with no visible inclusions.

Parallel: Lawall 2005, 47, fig. 6 (rouletted skyphos base).

\section{HELLENISTIC PAINTED WARE} (pls. 4-6, nos. 130-187)

This group is not a discrete group with common and definable characteristics, but it is clearly a continuation of the Late Iron Age painted ware tradition in the Hellenistic period with Greek forms and local painted features. We have 58 sherds in total, 45 of which are closed and 13 open forms. The form repertory of this ware is very limited: closed forms with short neck. It is noteworthy that most of the pieces are body sherds with $0.4-1.0 \mathrm{~cm}$ thickness so that it was difficult to assign them to any known Hellenistic forms. They seem to be pots for daily use such as storage vessels, or for religious purposes as urns.

Paste colors are reddish yellow (5YR 6/66/8, 7.5YR 6/6-7/6), light red (2.5YR 6/8-6/6), red (2.5YR 5/8-5/6), light brown (7.5YR 6/4), pink (5YR 7/4, 7.5YR 7/4), light reddish brown (5YR 6/4), very pale brown (10YR 7/4), yellowish red (5YR 5/6-5/8), pale brown (10YR 6/3), brown (7.5YR 5/3), reddish brown (5YR 5/3) and grey (7.5YR 6/1). Inclusions are lime, grog, sand, grit and micaceous in medium and small dimensions. Their generally hard paste is light and they are well-fired products. In some badly-fired products paste is grey or mottled. Slip colours differ as pink (5YR 7/4-8/3, 7.5YR 7/3-7/4-8/3), very pale brown (10YR 7/3-7/4-8/2-8/3-8/3), weak 
red $(5 \mathrm{R} 5 / 4,7.5 \mathrm{R} 4 / 3,4 / 4,10 \mathrm{R} 4 / 4-5 / 4)$ red $(5 \mathrm{R}$ 4/6, 7.5R 5/6, 10R 5/6, 2.5YR 5/6), white (10YR 8/1), reddish yellow (5YR 6/6, 7.5YR 6/6), light red (2.5YR 6/6), pale red (7.5R 6/4), light reddish brown (5YR 6/4), pinkish grey (5YR 7/2) and brown $(7.5$ YR $4 / 3)$.

The most important distinctive feature of this group is that the cream ground slip. The main decoration pattern is concentric bands in yellowish red (5YR 5/6), reddish brown (2.5YR 4/3, $5 \mathrm{YR} 5 / 3)$, pale red (7.5R 6/4) and weak red (10R $5 / 4)$ as well as red (5R 4/6, 10R 5/6-5/8, 2.5YR $5 / 6$ ). Slips are mostly shinny and smooth; some samples are polished. Decoration is similar to that of Galatian ware in some respects (cf. a sherd from Eskiyapar: Zoroğlu 1979, 214, fig. 6, draw. 5.). No distinctive Galatian ware, however, was found in southwestern Paphlagonia. Banded decoration is a widespread ceramic trend in Asia Minor during the Hellenistic period and is produced —or at least acquired and used — at sites between Eskişehir to the west and the Halys River to the east (Stewart 2010, 153). The particular manifestation of banded pottery that occurs on the Phrygian Plateau (bordered by Eskişehir to the west, the Halys River to the east, possibly the Black Sea coast to the north, and Çatalhöyük to the south) is homogeneous in terms of fabric, form, and decoration (Stewart 2010, 153).

25 of these sherds were found on the southern slope of the Acropolis at Kimistene, 3 fragments from the cistern of Kimistene. It seems that Hellenistic painted ware has a concentration at Kimistene. Only two sherds were collected from Kepez.

A crater fragment with similar decoration from Oluz Höyük (Dönmez 2010b, 63, fig. 102) is dated to the 4 th-3rd cent. B.C. In southwestern Paphlagonia, however, this group should be dated to the 3 rd-2nd centuries B.C.
OPEN FORMS (pl. 4, nos. 130-139) / Rim Fragments of Open Forms (pl. 4, nos. 130132)

Most of these sherds cannot be assigned to any known forms. No. 130 as well as 131 are similar forms in terms of rim profile.

130. (No. 1275): Rim fragment; Kimistene, Acropolis, southern slope, just below the summit, up to Deresemail creek, found in 2005. pl. 4/130 and pl. 32/130.

Max. h $1.3 \mathrm{~cm}$., d of rim $10.4 \mathrm{~cm}$., max. w 4.2 cm., max. th $0.6 \mathrm{~cm}$.

Very pale brown (10YR 8/2) slip on exterior; very pale brown (10YR 7/3) slip on lower interior. Two bands in very dark grey $(7.5 \mathrm{YR}$ $3 / 1)$ paint on exterior, in weak red (10R 5/4) paint on interior rim. Hard, non-porous, thin paste, fine, light brown (7.5YR 6/4) fabric with some sand inclusions.

131. (No. 544): Rim fragment; Kimistene, Acropolis, southern slope, just below the summit, up to Deresemail creek, found in 2005. pl. 4/131 and pl. 32/131.

Max. h 1.4 cm., d of rim $11.2 \mathrm{~cm}$., max. w 4.4 cm., max. th $0.8 \mathrm{~cm}$.

Very pale brown (10YR 8/2) slip on exterior; weak red $(10 \mathrm{R} 4 / 3)$ paint on interior. Two bands in dark brown (7.5YR 3/3) on exterior. Hard, non-porous, thin paste, fine, pink (7.5YR 7/4) fabric with some lime and sand inclusions.

132. (No. 916): Rim fragment; Kimistene, Acropolis, on a roughy area in southeastern terrace (on the weay to the Cistern), surface find, found in 2005. pl. 4/132 and pl. 32/132. Max. h $1.1 \mathrm{~cm}$., d of rim $27.4 \mathrm{~cm}$., max. w 5.9 cm., max. th $0.9 \mathrm{~cm}$.

Very pale brown (10YR 8/4) slip on exterior and interior. In weak red (7.5R 4/3) on interior 
rim. Hard, thin paste, sparsely porous, fired to light brown (7.5YR 6/4) fabric with occasional micaceous inclusions.

Flat based.

133. (No. 893): Base fragment; Kimistene, Acropolis, southern slope, underground cave, surface find, found in 2005. pl. 4/133 and pl. 32/133.

Max. h $2.0 \mathrm{~cm}$., d of base $4.4 \mathrm{~cm}$., max. w 3.0 cm., max. th $0.6 \mathrm{~cm}$.

Pink (7.5YR 7/3) slip on exterior, in weak red (10R 4/4) paint on exterior, red (2.5YR 5/6) slip on interior. Exterior surface is burnished. Hard, non-porous, thin paste, fine, light red (2.5YR $6 / 8$ ) fabric with some tiny lime inclusions.

Body Fragments of Open Forms (pl. 4, nos. 138-139)

134. (No. 902): Body fragment; Kimistene, Acropolis, southern slope, underground cave, surface find, found in 2005. pl. 32/134.

Max. h 1.6 cm., max. w $1.6 \mathrm{~cm}$., max. th 0.3 $\mathrm{cm}$.

Very pale brown (10YR 8/3) slip on exterior; reddish yellow (5YR 6/6) slip on interior. Two bands in weak red (10R 4/4) and very dark grey (5YR 3/1) on exterior. Interior surface is burnished. Hard, thin paste, non-porous, fine, reddish yellow (5YR 6/6) fabric with no visible inclusions.

135. (No. 1364): Body fragment; Kimistene, Acropolis, found in 2005. pl. 32/135.

Max. h 1.9 cm., max. w 2.4 cm., max. th 0.5 $\mathrm{cm}$.

Pink (7.5YR 7/4) slip on exterior; in red (10R $5 / 6)$ on interior. Two bands in black (7.5YR
2.5/1) on exterior. Its below part has been painted weak red (10R 4/4). Hard, thin paste, non-porous, fine, reddish yellow (5YR 6/6) fabric with occasional sand, frequent tiny lime inclusions.

136. (No. 812): Body fragment; Kimistene, Acropolis, southern slope, just below the summit, up to Deresemail creek, found in 2005. pl. 32/136.

Max. h $2.6 \mathrm{~cm}$., max. w $1.8 \mathrm{~cm}$., max. th 0.4 $\mathrm{cm}$.

Very pale brown (10YR 8/2) slip on exterior; reddish yellow (5YR 6/6) slip on interior. Four bands in red (10R 4/6) and very dark grey (5YR 3/1) on exterior. Interior surface is burnished. Hard, thin paste, non-porous, fine, reddish yellow (5YR 6/6) fabric with rare small lime inclusions.

137. (No. 853): Body fragment; Kimistene, Acropolis, southern slope, just below the summit, up to Deresemail creek, found in 2005. pl. 32/137.

Max. h 2.4 cm., max. w 2.5 cm., max. th 0.7 $\mathrm{cm}$.

Pink (7.5YR 7/4) slip on exterior and interior. Exterior surface is burnished. Three bands in dark brown (7.5YR 3/2) and red (10R 5/6) on interior. Hard, thin paste, very sparsely porous, fine, yellowish red (5YR 5/6) fabric with occasional small lime inclusions.

138. (No. 1066): Body fragment; Kimistene, Acropolis, southern slope, just below the summit, up to Deresemail creek, found in 2005. pl. 4/138 and pl. 32/138.

Max. h $1.5 \mathrm{~cm}$., max. w $2.1 \mathrm{~cm}$., max. th 0.5 $\mathrm{cm}$.

Reddish yellow (5YR 6/6) unslipped surface on exterior; very pale brown (10YR 8/4) slip on interior. Two bands in red (2.5YR 4/6) and very dark grey (5YR 3/1) paint on interior. 
Hard, thin paste, non-porous, fine, light red (2.5YR 6/8) fabric with no visible inclusions.

139. (No. 1023): Body fragment; Kepez, found in 2005. pl. 4/139 and pl. 32/139.

Max. h $1.3 \mathrm{~cm}$., max. w $3.4 \mathrm{~cm}$., max. th 0.8 $\mathrm{cm}$.

Very pale brown (10YR 7/3) slip on exterior; light reddish brown (5YR 6/4) slip on interior. Two bands in dusky red (10R 3/2) paint on interior. Average hardness; non-porous, thin paste, fine, reddish yellow (5YR 6/6) fabric with some tiny lime inclusions.

140. (No. 806): Body fragment; Kimistene, summit of the Acropolis, western slope, found in 2005. pl. 32/140.

Max. h $2.6 \mathrm{~cm}$., max. w $3.5 \mathrm{~cm}$., max. th 0.5 $\mathrm{cm}$.

Light red (2.5YR 6/6) unslipped surface on exterior; light red (2.5YR 6/6) slip on interior. Interior surface is burnished. On exterior red (2.5YR 5/6) horizontal bands. Interior surface is burnished. Average hardness; non-porous, thin paste, fine, yellowish red (5YR 5/8) fabric with frequent tiny lime inclusions.

141. (No. 514): Body fragment; Kimistene, Acropolis, southern slope, just below the summit, up to Deresemail creek, found in 2005. pl. 32/141.

Max. h $4.0 \mathrm{~cm}$., max. w $4.5 \mathrm{~cm}$., max. th 0.7 $\mathrm{cm}$.

Light brown (7.5YR 6/4) unslipped surface on exterior, light red (2.5YR 6/6) slip on interior. Brown (7.5YR 5/3) eroded on horizontal bands on exterior. Hard, thin paste, non-porous, fired to brown (7.5YR 5/3) fabric with some tiny lime and sand inclusions.

142. (No. 438): Body fragment; Kimistene, Acropolis, slope, found in 2005. pl. 33/142.

Max. h 4.0 cm., max. w $4.1 \mathrm{~cm}$., max. th 1.0 $\mathrm{cm}$.

Red (10R 5/6) smoothed slip on exterior; weak red (10R 5/4) slip on interior. A band in very dark grey (7.5YR 3/1) paint on interior. All of surface is burnished. Hard, thin paste, non-porous, fired to red (2.5YR 5/8) and reddish yellow (5YR 6/6) fabric with some tiny lime inclusions.

CLOSED FORMS (pls. 4-6, nos. 144187) / Rim Fragments (pl. 4, nos. 144-145)

No. 145 is narrow and short-necked. Most of the rim fragments are outcurved.

143. (No. 1047): Rim fragment; Kepez, found in 2005. pl. 33/143.

Max. h $2.0 \mathrm{~cm}$., max. w $3.5 \mathrm{~cm}$., max. th 0.6 $\mathrm{cm}$.

White (10YR 8/1) slip on exterior and interior rim. Pink (7.5YR 7/4) unslipped surface on lower interior. Average hardness; very sparsely porous, fine, reddish brown (5YR 6/6) fabric with frequent sand and inclusions.

144. (No. 1130): Rim fragment; Kimistene, Acropolis, found in 2005. pl. 4/144 and pl. 33/144.

Max. h 3.4 cm., max. w $2.7 \mathrm{~cm}$., max. th 0.7 $\mathrm{cm}$.

White (10YR 8/1) slip on exterior and interior rim. A band in very dark grey (7.5YR 3/1) on exterior. Hard, thin paste, very sparsely porous, fine, red (2.5YR 5/8) fabric with occasional sand inclusions.

145. (No. 881): Rim fragment; Kimistene, Acropolis, southern slope, underground cave, surface find, found in 2005. pl. 4/145 and pl. 33/145.

Max. h 3.1 cm., max. w 6.5 cm., max. th 0.8 $\mathrm{cm}$. 
Pink (7.5YR 7/3) slip on exterior; weak red $(10 \mathrm{R} 5 / 4)$ on interior rim. Its below part has very pale brown (10YR 7/3) unslipped surface on interior. A band in dusky red (10R 3/4) on exterior. Hard, non-porous, thin paste, fine, light brown (7.5YR 6/4) fabric with some tiny lime inclusions.

\section{Base Fragment of Closed Form (pl. 4, no.} 146)

146. (No. 1478): Base fragment of a closed form; Kimistene, Acropolis, found in 2005. pl. 4/146 and pl. 33/146.

Max. h $3.6 \mathrm{~cm}$., d of base $8.6 \mathrm{~cm}$., max. w 5.3 $\mathrm{cm}$., max. th $0.7 \mathrm{~cm}$.

Pinkish grey (5YR 7/2) slip on the exterior; reddish yellow (5YR 6/6) unslipped surface on the interior. Two parallel horizontal bands on the exterior. The upper and lower bands are weak red (5R $4 / 3$ or $5 R 4 / 2)$. Hard, nonporous, thin paste, fine, light red (2.5YR 6/6) fabric with some tiny lime and sand inclusions. High-based form with internal wheelmarks.

Body Fragments of Closed Form (pls. 4-6, nos. 148-187)

Large carinated forms. No ornemantations. Some of them are polished; some of them have strong internal wheel-marks.

147. (No. 897): Body fragment; Kimistene, Acropolis, southern slope, underground cave, surface find, found in 2005. pl. 33/147.

Max. h $2.1 \mathrm{~cm}$., max. w $2.0 \mathrm{~cm}$., max. th 0.5 $\mathrm{cm}$.

Weak red (7.5R 4/4) slip on exterior; reddish yellow (5YR 6/6) unslipped surface on interior. Exterior surface is burnished. Hard, nonporous, thin paste, fine, light red (2.5YR 6/8) fabric with some tiny lime inclusions.
148. (No. 442): Body fragment; Kimistene, Acropolis, slope, found in 2005. pl. 4/148 and pl. 33/148.

Max. h 1.8 cm., max. w 2.3 cm., max. th 0.6 $\mathrm{cm}$.

Pink (5YR 8/3) slip on exterior; reddish yellow (5YR 7/6) unslipped surface on interior. Two bands in black (5YR 2.5/1) paint on upper exterior. Its below part has weak red (10R 4/4) paint. Average hardness; thin paste, very sparsely porous, fine, light red (2.5YR 6/8) fabric with frequent tiny lime and some sand inclusions.

149. (No. 950): Body fragment; Kimistene, Acropolis, southern slope, just below the summit, up to Deresemail creek, found in 2005. pl. 33/149.

Max. h 1.7 cm., max. w $3.5 \mathrm{~cm}$., max. th 0.5 $\mathrm{cm}$.

Red (7.5R 5/6) abrasion slip on exterior; pink (7.5YR 7/4) unslipped surface on interior. Hard, thin paste, porous, fine, pink (7.5YR $7 / 4$ ) fabric with some tiny lime inclusions.

150. (No. 1371): Body fragment; Kimistene, Acropolis, southern slope, just below the summit, up to Deresemail creek, found in 2005. pl. 33/150.

Max. h 2.3 cm., max. w 3.0 cm., max. th 0.4 $\mathrm{cm}$.

Weak red (10R 5/4) slip on exterior; pink (7.5YR 7/4) unslipped surface on interior. Hard, thin paste, very sparsely porous, fine, pink (7.5YR 7/4) fabric with no visible inclusions.

151. (No. 1149): Body fragment; Kimistene, Acropolis, northern slope, found in 2005. pl. 33/151.

Max. h $2.8 \mathrm{~cm}$., max. w $2.3 \mathrm{~cm}$., max. th 0.8 $\mathrm{cm}$.

Weak red (10R 5/4) slip on exterior; pink 
(7.5YR 7/4) unslipped surface on interior. Average hardness; non-porous, fired to reddish yellow (5YR 6/6) fabric with frequent tiny lime and micaceous inclusions.

152. (No. 1325): Body fragment; Kimistene, summit of the Acropolis, southern slope, found in 2005. pl. 33/152.

Max. h $1.7 \mathrm{~cm}$., max. w $3.1 \mathrm{~cm}$., max. th 0.6 $\mathrm{cm}$.

Very pale brown (10YR 7/4) slip on exterior; reddish yellow (7.5YR 7/6) unslipped surface on interior. Two bands in reddish brown (5YR $5 / 4)$ and very dark grey (10YR 3/1) on exterior. Average hardness; thin paste, non-porous, fired to reddish yellow (7.5YR 7/6-5YR 6/8) fabric with rare tiny lime inclusions.

153. (No. 441): Body fragment; Kimistene, Acropolis, slope, found in 2005. pl. 33/153.

Max. h 1.9 cm., max. w $3.3 \mathrm{~cm}$., max. th 0.9 $\mathrm{cm}$.

Red (2.5YR 5/6) slip on exterior; reddish yellow (5YR 6/6) unslipped surface on interior. A band in dark reddish grey (2.5YR 3/1) on exterior. Hard, non-porous, thin paste, fine, reddish yellow (5YR 6/6) fabric with occasional tiny lime inclusions.

154. (No. 1262): Body fragment; Kimistene, Acropolis, southern slope, just below the summit, up to Deresemail creek, found in 2005. pl. 33/154.

Max. h $2.5 \mathrm{~cm}$., max. w $2.9 \mathrm{~cm}$., max. th 0.5 $\mathrm{cm}$.

Pink (7.5YR 8/3) slip on exterior; very pale brown (10YR 8/4) unslipped surface on interior. Two bands in dark brown (7.5YR 3/2) on exterior. These bands are bordered with two bands in reddish brown (2.5YR 4/3). Average hardness; thin paste, non-porous, fine, very pale brown (10YR 7/4) fabric with rare sand and micaceous inclusions.
155. (No. 1235): Body fragment; Kimistene, Acropolis, southern slope, just below the summit, up to Deresemail creek, found in 2005. pl. 33/155.

Max. h 2.9 cm., max. w 2.7 cm., max. th 0.6 cm.

Pink (7.5YR 8/3) slip on exterior; reddish yellow (5YR 6/6) unslipped surface on interior. Three bands in red (2.5YR 5/6) and dark reddish grey (5YR 4/2) on exterior. Hard, thin paste, very sparsely porous, fine, light brown (7.5YR 6/4) fabric with some sand inclusions.

156. (No. 972): Body fragment; Kimistene, Acropolis, southern slope, just below the summit, up to Deresemail creek, found in 2005. pl. 4/156 and pl. 33/156.

Max. h $2.2 \mathrm{~cm}$., max. w $3.4 \mathrm{~cm}$., max. th 0.5 $\mathrm{cm}$.

Pink (7.5YR 8/3) slip on exterior; light red (2.5YR 6/8) unslipped surface on interior. Two bands in very dark grey (7.5YR 3/1) paint on exterior. Hard, thin paste, non-porous, fine, red (2.5YR 5/8) fabric with rare sand, occasional tiny lime inclusions.

157. (No. 1011): Body fragment; Kimistene, Acropolis, slope, found in 2005. pl. 33/157.

Max. h $2.5 \mathrm{~cm}$., max. w $3.6 \mathrm{~cm}$., max. th 0.8 $\mathrm{cm}$.

Weak red (5R 5/4) slip on exterior; reddish yellow (5YR 6/6) unslipped surface on interior. Average hardness; non-porous, thin paste, fine, light red (2.5YR 6/8) fabric with no visible inclusions.

158. (No. 940): Body fragment; Kimistene, Acropolis, found in 2005. pl. 33/158.

Max. h $2.8 \mathrm{~cm}$., max. w $2.8 \mathrm{~cm}$., max. th 0.7 $\mathrm{cm}$.

Red (10R 5/6) shiny slip on exterior; light red (2.5YR 6/6) unslipped surface on interior. Exterior surface is burnished. Hard, thin paste, 
very sparsely porous, fine, red (2.5YR 5/8) fabric with some tiny lime inclusions.

159. (No. 1397): Body fragment; Kimistene, summit of the Acropolis, surface find, found in 2005. pl. 33/159.

Max. h $2.9 \mathrm{~cm}$., max. w $4.6 \mathrm{~cm}$., max. th 0.4 $\mathrm{cm}$.

Weak red (7.5R 4/3) slip on exterior; reddish yellow (7.5YR 7/6) unslipped surface on interior. Hard, thin paste, non-porous, fired to reddish yellow (5YR 6/8) and reddish yellow (5YR 6/6) fabric with no visible inclusions.

160. (No. 1351): Body fragment; Kimistene, Cistern, eastern slope, found in 2005. pl. 33/160.

Max. h 3.4 cm., max. w $3.2 \mathrm{~cm}$., max. th 0.5 $\mathrm{cm}$.

Light reddish brown (5YR 6/3) unslipped surface; reddish yellow (5YR 6/6) unslipped surface on interior. Traces of a band in reddish brown (5YR 5/4). Hard, thin paste, very sparsely porous, fine, reddish yellow (5YR 6/8) fabric with some micaceous and sand inclusions.

161. (No. 416): Body fragment; Kimistene, Necropolis 1, found in 2005. pl. 4/161 and pl. 33/161.

Max. h 2.9 cm., max. w $3.3 \mathrm{~cm}$., max. th 0.8 $\mathrm{cm}$.

Very pale brown (10YR 7/4) slip on exterior, light red (2.5YR 6/8) unslipped surface on interior. Two bands in very dark brown (7.5YR $3 / 2$ ) paint on exterior surface. Its below part has a red (2.5YR 5/6) paint. Average hardness; non-porous, fired to red (2.5YR 5/8) and pale brown (10YR 6/3) fabric with frequent tiny lime and sand inclusions.

162. (No. 1285): Body fragment; Kimistene, Acropolis, on the craggy slope to the Acro- pole, found in 2005. pl. 5/162 and pl. 33/162. Max. h $3.1 \mathrm{~cm}$., max. w $2.7 \mathrm{~cm}$., max. th 0.5 $\mathrm{cm}$.

Very pale brown (10YR 7/4) slip on exterior; very pale brown (10YR 7/4) unslipped surface on interior. Two bands in very dark grey (5YR 3/1). Its below part has yellowish red (5YR 5/6) on exterior. Hard, thin paste, very sparsely porous, fine, very pale brown (10YR 7/4) fabric with rare lime inclusions.

163. (No. 889): Body and base fragment; Kimistene, Acropolis, southern slope, underground cave, surface find, found in 2005. pl. 33/163.

Max. h 2.9 cm., max. w $4.2 \mathrm{~cm}$., max. th 0.7 $\mathrm{cm}$.

Weak red (10R 4/4) slip on upper exterior; pink (7.5YR 7/4) slip around the exterior base. Reddish yellow (5YR 7/6) unslipped surface on interior. Hard, thin paste, non-porous, fine, reddish yellow (5YR 6/8) fabric with no visible inclusions.

164. (No. 537): Body fragment; Kimistene, Acropolis, southern slope, just below the summit, up to Deresemail creek, found in 2005. pl. 5/164 and pl. 33/164.

Max. h $3.8 \mathrm{~cm}$., max. w $3.0 \mathrm{~cm}$., max. th 0.4 $\mathrm{cm}$.

Pink (7.5YR 8/3) slip on exterior; reddish yellow (5YR 7/6) unslipped surface on interior. Three bands in very dark grey (7.5YR 3/1) on exterior. Its below part has reddish brown (2.5YR 4/3). Hard, thin paste, non-porous, fired to reddish yellow (5YR 6/8) fabric with no visible inclusions.

165. (No. 407): Body fragment; Necropolis 1 , found in 2005. pl. 5/165 and pl. 33/165.

Max. h $1.8 \mathrm{~cm}$., max. w $3.4 \mathrm{~cm}$., max. th 0.4 $\mathrm{cm}$.

Very pale brown (10YR 8/2) slip on exte- 
rior; light red (2.5YR 6/8) unslipped surface on interior. Three bands in black (7.5YR $2.5 / 1)$ paint on exterior. Hard, thin paste, very sparsely porous, fine, light red (2.5YR 6/8) fabric with no visible inclusions.

166. (No. 1242): Body fragment; Kimistene, Acropolis, southern slope, just below the summit, up to Deresemail creek, found in 2005. pl. 5/166 and pl. 33/166.

Max. h $3.3 \mathrm{~cm}$., max. w $2.6 \mathrm{~cm}$., max. th 0.7 $\mathrm{cm}$.

Red (2.5YR 5/6) slip on exterior, pink (7.5YR 7/4) unslipped surface on interior. A band in dark reddish grey $(2.5 \mathrm{YR} 3 / 1)$ paint on upper exterior. Hard, thin paste, very sparsely porous, fired to pink (7.5YR 7/4) and reddish yellow (5YR 6/6) fabric with occasional sand and tiny lime inclusions.

167. (No. 400): Body fragment; Kimistene, Acropolis, eastern (lowest) slope; found in 2005. pl. 5/167 and pl. 33/167.

Max. h $2.2 \mathrm{~cm}$., max. w $4.2 \mathrm{~cm}$., max. th 0.5 $\mathrm{cm}$.

Very pale brown (10YR 8/3) slip on exterior; reddish yellow (5YR 7/6) unslipped surface on interior. A band in very dark grey (10YR $3 / 1$ ) paint on exterior; its below part has weak red (10R 5/4) on exterior slip. Average hardness; thin paste, very sparsely porous, fine, reddish yellow (5YR 6/8) fabric with occasional tiny lime inclusions.

168. (No. 731): Body fragment; Kimistene, Acropolis, temple's terrace, illegally excavated area under the temple's podium, found in 2005. pl. 5/168 and pl. 33/168.

Max. h $3.1 \mathrm{~cm}$., max. w $4.6 \mathrm{~cm}$., max. th 0.5 $\mathrm{cm}$.

Light reddish brown (2.5YR 6/4) unslipped surface on exterior; light brown (7.5YR 6/4) unslipped surface on interior. A band in very dark grey (5YR 3/1) paint on exterior. Hard, thin paste, non-porous, fine, red (2.5YR 5/6) fabric with rare lime and sand inclusions.

169. (No. 934): Body fragment of a closed form; Kimistene, Acropolis, found in 2005. pl. 5/169 and pl. 33/169.

Max. h $2.3 \mathrm{~cm}$., max. w $5.7 \mathrm{~cm}$., max. th 0.8 $\mathrm{cm}$.

White (10YR 8/1) slip on the exterior; light reddish brown (5YR 6/3) unslipped surface on the interior. Two parallel horizontal bands on the exterior; these bands are in dark reddish grey $(2.5 \mathrm{YR} 4 / 1)$. The lower part is in reddish brown (5YR 5/3). Hard, non-porous, thin paste, fired to reddish brown (5YR 5/3) fabric with some micaceous and sand inclusion.

170. (No. 444): Body fragment; Kimistene, Acropolis, slope, found in 2005. pl. 5/170 and pl. 33/170.

Max. h $3.4 \mathrm{~cm}$., max. w $2.7 \mathrm{~cm}$., max. th 0.6 $\mathrm{cm}$.

Pink (7.5YR 7/4) slip on exterior; reddish yellow (7.5YR 7/6) unslipped surface on interior. A band in very dark grey (7.5YR 3/1) paint on exterior. Hard, very sparsely porous, fine, light brown (7.5YR 6/4) fabric with rare lime inclusions.

171. (No. 1360): Body fragment; Kimistene, Cistern, eastern slope, found in 2005. pl. 5/171 and pl. 33/171.

Max. h 3.6 cm., max. w $2.8 \mathrm{~cm}$., max. th 0.6 $\mathrm{cm}$.

Pink (7.5YR 7/3) slip on exterior; pink (7.5YR $7 / 3$ ) unslipped surface on interior. A band in very dark grey (5YR 3/1) paint on exterior, its below part has red (10R 5/6) on exterior slip. Hard, thin paste, very sparsely porous, fine, pink (7.5YR 7/4) fabric with occasional micaceous and sand inclusions. 
172. (No. 1350): Body fragment; Kimistene, Cistern, eastern slope, found 2005. pl. 5/172 and pl. 33/172.

Max. h 3.4 cm., max. w 3.7 cm., max. th 0.8 $\mathrm{cm}$.

Red (5R 4/6) slip on exterior; reddish yellow (5YR 6/6) unslipped surface on interior. A band in dark reddish grey (2.5YR 3/1) paint on upper exterior. Exterior surface is burnished. Hard, thin paste, non-porous, fine, red (2.5YR 5/8) fabric with some tiny lime inclusions.

173. (No. 524): Body fragment; Kimistene, Acropolis, southern slope, just below the summit, up to Deresemail creek, found in 2005.

pl. 5/173 and pl. 33/173.

Max. h 3.8 cm., max. w $4.0 \mathrm{~cm}$., max. th 0.7 $\mathrm{cm}$.

Pink (7.5YR 8/3) slip on exterior, pink (7.5YR 7/4) unslipped surface on interior. Two bands in very dark grey $(7.5 \mathrm{YR} 3 / 1)$ on exterior. Hard, thin paste, very sparsely porous, fine, reddish yellow (7.5YR 7/6) fabric with occasional tiny lime inclusions.

174. (No. 939): Body fragment; Kimistene, Acropolis, found in 2005. pl. 5/174 and pl. 34/174.

Max. h $2.6 \mathrm{~cm}$., max. w $4.2 \mathrm{~cm}$., $\max$. th 0.8 $\mathrm{cm}$.

Red (10R 5/6) shiny slip on exterior; pink (7.5YR 7/4) unslipped surface on interior. A band in very dark grey (5YR 3/1) paint on exterior. Exterior surface is burnished. Hard, very sparsely porous, thin paste, fine, red (2.5YR 5/8) fabric with some micaceous, rare lime and grog less than $0.5 \mathrm{~cm}$. inclusions.

175. (No. 546): Body fragment; Kimistene, Acropolis, southern slope, just below the summit, up to Deresemail creek, found in 2005. pl. 5/175 and pl. 34/175.
Max. h 2.9 cm., max. w $4.8 \mathrm{~cm}$., max. th 0.7 $\mathrm{cm}$.

Red (2.5YR 5/6) slip on lower exterior; pink (7.5YR 7/4) unslipped surface on interior. A band in very dark grey (5YR 3/1) on upper exterior. Exterior surface is burnished. Hard, thin paste, non-porous, fired to light brown (7.5YR 6/4) and grey (7.5YR 6/1) fabric with frequent tiny lime and rare sand inclusions.

176. (No. 398): Body fragment; Kimistene, Acropolis, eastern (lowest) slope, found in 2005. pl. 5/176 and pl. 34/176.

Max. h $4.8 \mathrm{~cm}$., max. w $4.2 \mathrm{~cm}$., max. th 0.5 $\mathrm{cm}$.

Reddish yellow (7.5YR 7/6) slip on exterior; pink (5YR 7/4) unslipped surface on interior. Interior surface is smoothed. Three bands in black (7.5YR 2.5/1) on exterior, its below part in red (2.5YR 5/6). Hard, thin paste, very sparsely porous, fine, light reddish brown (5YR 6/4) fabric with occasional tiny lime inclusions.

177. (No. 924): Body fragment of a closed form; Kimistene, Acropolis, found in 2005. pl. 5/177 and pl. 34/177.

Max. h $2.3 \mathrm{~cm}$., max. w $5.2 \mathrm{~cm}$., max. th 0.8 $\mathrm{cm}$.

Pink (7.5YR 8/3) slip on the exterior; pink (5YR 7/4) unslipped surface on the interior. On the exterior there are two parallel dark reddish grey (10R 4/1) horizontal bands. Its lower part is in red (10R 5/8). Hard, thin paste, non-porous, fine, pink (5YR 7/4) fabric with some micaceous and lime inclusion.

178. (No. 919): Body fragment; Kimistene, Acropolis, southeastern slope; surface find, found in 2005. pl. 34/178.

Max. h $4.1 \mathrm{~cm}$., max. w $4.1 \mathrm{~cm}$., max. th 0.6 $\mathrm{cm}$.

Very pale brown (10YR 8/2) slip on exterior; 
reddish yellow (7.5YR 7/6) unslipped surface on interior. A band in very dark grey $(7.5 \mathrm{YR}$ $3 / 1$ ) on upper exterior. Exterior surface is burnished. Hard, thin paste, very sparsely porous, fine, reddish yellow (7.5YR 6/6) fabric with no visible inclusions.

179. (No. 1453): Body fragment; uncertain. pl. 34/179.

Max. h $4.5 \mathrm{~cm}$., max. w $4.9 \mathrm{~cm}$., max. th 0.6 $\mathrm{cm}$.

Weak red (10R 4/4) matt slip on exterior; pink (5YR 7/4) unslipped surface on interior. Hard, thin paste, very sparsely porous, fine, reddish yellow (5YR 6/6) fabric with no visible inclusions.

180. (No. 1237): Body fragment; Kimistene, Acropolis, southern slope, just below the summit, up to Deresemail creek, found in 2005. pl. 5/180 and pl. 34/180.

Max. h $4.8 \mathrm{~cm}$., max. w $4.6 \mathrm{~cm}$., max. th 0.6 $\mathrm{cm}$.

Very pale brown (10YR 8/2) abrasion slip on exterior; pink (7.5YR 7/4) unslipped surface on interior. Three bands in yellowish red (5YR 5/6) on exterior. Average hardness; non-porous, thin paste, fine, light brown (7.5YR 6/4) fabric with occasional sand and tiny lime inclusions.

181. (No. 887): Body fragment; Kimistene, Acropolis, southern slope, underground cave, surface find, found in 2005. pl. 5/181 and pl. 34/181.

Max. h $2.5 \mathrm{~cm}$., max. w $5.8 \mathrm{~cm}$., max. th 1.0 $\mathrm{cm}$.

Pink (7.5YR 7/4) unslipped surface on exterior; pink (7.5YR 8/4) unslipped surface on interior. Two bands in very dark grey (5YR $3 / 1$ ) paint on exterior. Hard, thin paste, nonporous, fired to light reddish brown (5YR 6/4) and light red (2.5YR 6/8) fabric with rare tiny lime inclusions.
182. (No. 854): Body fragment; Kimistene, Acropolis, northwestern slope of the Temple, found in 2005. pl. 34/182.

Max. h 4.6 cm., max. w $5.1 \mathrm{~cm}$., max. th 0.9 $\mathrm{cm}$.

Weak red (10R 4/4) abrasion slip on exterior; light red (2.5YR 6/6) unslipped surface on interior. Exterior surface is burnished. Hard, thin paste, non-porous, fine, red (2.5YR 5/6) fabric with some tiny lime inclusions.

183. (No. 434): Body fragment; Kimistene, Acropolis, slope, found in 2005. pl. 5/183 and pl. 34/183.

Max. h $4.0 \mathrm{~cm}$., max. w $4.1 \mathrm{~cm}$., max. th 0.7 $\mathrm{cm}$.

Pale red (7.5R 6/4) slip on lower exterior; reddish yellow (7.5YR 7/6) unslipped surface on interior. A band in dark reddish grey $(2.5 \mathrm{YR}$ $3 / 1$ ) paint on upper exterior. Hard, thin paste, fine, light red (2.5YR 6/8) fabric with some tiny lime inclusions.

184. (No. 439): Body fragment; Kimistene, Acropolis, slope, found in 2005. pl. 34/184.

Max. h $4.3 \mathrm{~cm}$., max. w $4.2 \mathrm{~cm}$., max. th 1.2 $\mathrm{cm}$.

Weak red (10R 5/4) slip on exterior; reddish yellow (7.5YR 7/6) unslipped surface on interior. Hard, thin paste, non-porous, fine, pink (7.5YR 7/4) fabric with rare tiny lime inclusions.

185. (No. 771): Body fragment; Kimistene, Acropolis, southern slope, just below the summit, up to Deresemail creek, found in 2005. pl. 34/185.

Max. h $5.7 \mathrm{~cm}$., max. w $5.2 \mathrm{~cm}$., max. th 0.8 $\mathrm{cm}$.

White (10YR 8/1) slip on exterior; pink (5YR 7/4) unslipped surface on upper interior. Pink (5YR 7/4) slip on the lower part on interior. Interior slip is separated with a band in red 
(2.5YR 5/6) paint. Hard, thin paste, non-porous, fired to reddish yellow (5YR 6/6) fabric with occasional medium grit and rare grog inclusions.

186. (No. 443): Body fragment; Kimistene, Acropolis, slope, found in 2005. pl. 6/186 and pl. 34/186.

Max. h 6.0 cm., max. w 6.9 cm., max. th 1.2 $\mathrm{cm}$.

Weak red (10R 5/4) slip on exterior; pink (7.5YR 7/4) unslipped surface on interior. A band in very dark grey (7.5YR 3/1) paint on upper exterior. Hard, thin paste, porous, fine, pink (7.5YR 7/4) fabric with some tiny lime and sand inclusions.

187. (No. 525): Body fragment; Kimistene, Acropolis, southern slope, just below the summit, up to Deresemail creek, found in 2005. pl. 6/187 and pl. 34/187.

Max. h $6.1 \mathrm{~cm}$., max. w $7.3 \mathrm{~cm}$., max. th 0.9 $\mathrm{cm}$.

Very pale brown (10YR 8/2) slip on upper exterior; very pale brown (10YR 7/4) unslipped surface on interior. A band in dark reddish grey $(2.5 \mathrm{YR} 4 / 1)$ paint on upper exterior; the lower part has red (2.5YR 5/6) on exterior. Exterior surface is burnished. Hard, thin paste, very sparsely porous, fine, light reddish brown (5YR 6/4) fabric with some lime inclusions.

\section{HELLENISTIC RELIEF WARE} (pl. 6, nos.188-201)

This mould-made ware has only one form, the bowl. In these bowls the exterior surface is decorated with high relief. At the exterior surface Ionic kymation as well as degenerated floral decoration were applied, perhaps through the influence of external workshops in western Asia
Minor. Their rims are outcurved and body form must be globular. 14 fragments were collected, 12 of which are from Kepez and two from Kimistene. Paste is reddish yellow (7.5YR 6/6, 5YR 6/6), light brown (7.5YR 6/4), pale brown (10YR $6 / 3)$, very pale brown (10YR 7/4), pale red (10R $6 / 2$ ) and yellowish red (5YR 5/6). Inclusions are tiny lime, quartz, micaceous and sand small grit. Inclusions are small. Paste is hard and less porous. Slip is red (2.5YR 5/6-4/6-5/8, 10R 5/6), light red (2.5YR 6/6), reddish yellow (5YR 6/6-7/6), reddish brown (2.5YR 5/4, 5YR 4/4-5/4) and dark brown (7.5YR 3/2).

Some relief ware examples were also collected in Matthews's surveys of Inner Paphlagonia (Matthews/Metcalfe/Cottica 2009, 220, fig. 6.104, nos. 11-14 (site PS 168).

2nd-1st century B.C.

OPEN FORMS (pl. 6, nos. 188-201) / Rim Fragments of a Bowl Form (pl. 6, nos. 188190)

Some of them bear a rim frieze with Ionian kymation (parallels: Bilde 2010, 276, pl. 169, F-6, F-8, F-9).

188. (No. 1091): Rim fragment; Kepez, found in 2005. pl. 6/188 and pl. 34/188.

Max. h $4.7 \mathrm{~cm}$., max. w $4.4 \mathrm{~cm}$., max. th 0.7 cm.

Everted rim; horizontal leaf-decoration. Red (2.5YR 5/6) slip on the exterior; light red (2.5YR 6/6) slip on the interior. Average hardness; thin paste; very sparsely porous, fine, reddish yellow (5YR 6/6) and light red (2.5YR 6/8) mottled fabric with some lime and rare small grit inclusion.

189. (No. 1090): Rim fragment; Kepez, found in 2005. pl. 6/189 and pl. 34/189.

Max. h 3.9 cm., d of rim $14.8 \mathrm{~cm}$., max. w 4.3 
cm., max. th $0.6 \mathrm{~cm}$.

Everted rim; in the rim zone, a band of eggand-dart pattern below the rim (similar to: Edwards 1956, 99, pl. 42, nos. 58a-b). Light red (2.5YR 6/6) matt slip on the exterior and interior. Hard, thin paste; very sparsely porous, fine, reddish yellow (7.5YR 6/6) fabric with frequent tiny lime inclusions.

Parallel: Chalier 2008, 96-97, 3a-b, 4a.

190. (No. 1283): Rim fragment; Kimistene, Necropolis 1, found in 2005. pl. 6/190 and pl. 34/190.

Max. h $3.2 \mathrm{~cm}$., d of rim $15.4 \mathrm{~cm}$., max. w 4.2 cm., max. th $0.6 \mathrm{~cm}$.

Everted rim, red (2.5YR 5/6) matt, thick slip on exterior; red (2.5YR 5/6) on interior. A band in pink (5YR 7/3) paint on exterior rim. Average hardness; non-porous, thin paste, fine, reddish yellow (7.5YR 6/6) fabric with some tiny lime inclusions.

Parallel: Chalier 2008, 96-97, 3a-b, 4a.

Body Fragments of Open Forms (pl. 6, nos. 196-201)

191. (No. 1026): Body fragment; Kepez, found in 2005. pl. 34/191.

Max. h 1.8 cm., max. w 2.9 cm., max. th 0.6 $\mathrm{cm}$.

Red (2.5YR 4/6) slip on exterior; light red (2.5YR 6/6) slip on interior. Average hardness; thin paste, non-porous, fine, light brown (7.5YR 6/4) fabric with occasional small grit inclusions.

192. (No. 1030): Body fragment; Kepez, found in 2005. pl. 34/192.

Max. h $2.7 \mathrm{~cm}$., max. w $2.3 \mathrm{~cm}$., max. th 0.5 $\mathrm{cm}$.

Reddish brown (5YR 4/4) slip on exterior; red (2.5YR 5/6) slip on interior. Two bands in pinkish white (7.5YR 8/2) and dark reddish brown (5YR 3/3) paint on exterior. Hard, thin paste, non-porous, fine, pale brown (10YR $6 / 3$ ) fabric with some tiny lime and rare sand inclusions.

193. (No. 1027): Body fragment; Kepez, found in 2005. pl. 34/193.

Max. h 2.4 cm., max. w 2.7 cm., max. th 0.6 $\mathrm{cm}$.

Red (2.5YR 5/8) slip on exterior and interior. Hard, thin paste, non-porous, fired to reddish yellow (5YR 6/6) fabric with some lime and sand, rare micaceous inclusions.

194. (No. 1054): Body fragment; Kepez, found in 2005. pl. 34/194.

Max. h $3.2 \mathrm{~cm}$., max. w $2.2 \mathrm{~cm}$., max. th 0.7 $\mathrm{cm}$.

Red (2.5YR 5/6) slip on exterior; very pale brown (10YR 7/3) unslipped surface on interior. Soft, thin paste, non-porous, fine, pale brown (10YR 6/3) fabric with rare lime inclusions.

195. (No. 1017): Body fragment; Kepez, found in 2005. pl. 34/195.

Max. h $1.8 \mathrm{~cm}$., max. w $3.1 \mathrm{~cm}$., max. th 0.5 $\mathrm{cm}$.

Reddish brown (2.5YR 5/4) slip on exterior; red (2.5YR 5/6) tiny slip on interior. Average hardness; thin paste, non-porous, fine, reddish yellow (7.5YR 6/6) fabric with no visible inclusions.

196. (No. 1019): Body fragment; Kepez, found in 2005. pl. 6/196 and pl. 34/196.

Max. h 3.4 cm., max. w $3.1 \mathrm{~cm}$., max. th 0.5 $\mathrm{cm}$.

Reddish yellow (5YR 6/6) slip on exterior; reddish yellow (5YR 7/6) slip on interior. Small leaf ornamentation on exterior surface. Hard, thin paste, non-porous, very pale brown (10YR 7/4) and light brownish grey (10YR 
6/2) fabric with infrequent lime and sand inclusions.

197. (No. 921): Body fragment; Kimistene, Acropolis, southeastern slope, on the way to the Cistern, on a rocky slope, surface find, found in 2005. pl. 6/197 and pl. 35/197.

Max. h $2.8 \mathrm{~cm}$., max. w $3.9 \mathrm{~cm}$., max. th 0.6 $\mathrm{cm}$.

Dark brown (7.5YR 3/2) slip on exterior; red (10R 5/6) slip on interior. Average hardness; thin paste, non-porous, fine, reddish yellow (7.5YR 6/6) fabric with infrequent tiny lime inclusions.

198. (No. 1231): Body fragment; Kepez, found in 2005. pl. 6/198 and pl. 35/198.

Max. h 2.4 cm., max. w $3.5 \mathrm{~cm}$., max. th 0.7 $\mathrm{cm}$.

Reddish brown (5YR 5/4) slip on exterior and interior. Small leaf ornamentation on exterior surface. Average hardness; thin paste, sparsely porous, fine, yellowish red (5YR 5/6) fabric with rare sand, frequent tiny lime inclusions.

199. (No. 1001): Body fragment; Kepez, found in 2005. pl. 6/199 and pl. 35/199.

Max. h 2.1 cm., max. w 4.9 cm., max. th 0.8 $\mathrm{cm}$.

Red (2.5YR 5/6) slip on exterior and interior. Hard, thin paste, very sparsely porous, fine, light brown (7.5YR 6/4) fabric with rare quartz and a little micaceous inclusions.

Raised pattern of rays spreading vertically from bottom of bowl. Each ray consists of one lines. In the space between the rays, series of raised dots are set in vertical rows.

200. (No. 1003): Body fragment; Kepez, found in 2005. pl. 6/200 and pl. 35/200.

Max. h 3.2 cm., max. w $5.8 \mathrm{~cm}$., max. th 0.7 $\mathrm{cm}$.

Red (2.5YR 5/6) tiny slip on exterior and interior. Hard, thin paste, non-porous, fine, reddish yellow (7.5YR 6/6) fabric with infrequent tiny lime inclusions.

201. (No. 1006): Body fragment; Kepez, found in 2005. pl. 6/201 and pl. 35/201.

Max. h $2.4 \mathrm{~cm}$., max. w $6.6 \mathrm{~cm}$., max. th 0.8 $\mathrm{cm}$.

Reddish yellow (5YR 6/6) slip on exterior; its upper part is very pale brown (10YR 8/3) slip; red (2.5YR 5/6) slip on interior. Average hardness; porous, thin paste, fine, light brown (7.5YR 6/4) fabric with occasional tiny lime inclusions.

\section{HELLENISTIC BURNISHED} WARE (pls. 7-9, nos. 202-256)

Major forms are incurved rim bowls and fish plates. These are the imitations of some major Hellenistic forms of western Asia Minor. The incurving rim bowl is the most common shape in the Middle Hellenistic period in the whole landscape. These bowls should be drinking vessels.

Paste is reddish yellow (7.5YR 7/6-6/6, 5YR 6/8-6/6-7/6), light brown (7.5YR 6/4), pale brown (10YR 6/3), yellowish red (5YR 5/6), brown $(7.5 \mathrm{YR} 5 / 4,10 \mathrm{YR} 5 / 3)$, very pale brown (10YR 7/3-7/4), pink (7.5YR 7/4), light red (2.5YR 6/8), red (2.5YR 5/8), reddish brown (5YR 5/4) and light yellowish brown (10YR 6/4). Hard paste, non-porous and well fired. Bad paste became mottled in grey (7.5YR 6/1), dark grey (7.5YR 4/1) and black (2.5Y 2.5/1). Inclusions are sand, micaceous, small grit, grog, and tiny lime in medium dimensions. Some of the samples are without any inclusions. Their thickness differ between $0.5-1.0 \mathrm{~cm}$. Thin slip mostly in the same colour of paste, ranging from very pale brown (10YR 8/2-8/3-8/4-7/3-7/4) to reddish yellow (5YR 6/6, 7.5YR 6/6-7/6), pink (7.5YR 7/4-7/38/4, 5 YR 7/4), light yellowish brown (10YR 6/4), 
pale brown (10YR 6/3), light grey (10YR 7/2), reddish brown (5YR 5/4), yellowish red (5YR $5 / 6)$, pinkish white $(7.5 \mathrm{YR} 8 / 2)$ and red $(2.5 \mathrm{YR}$ 5/6). Slip was applied on light coloured-paste and polished slightly. Most of them have no decoration, except on no. 250 where there is engraved linear decoration on its exterior. In exterior wall burnished surface and wheel-marks.

There are 60 fragments in total; 48 of them belong to open and 12 to closed forms. 34 fragments were found at Kepez, and 11 on the southern slope of Acropolis at Kimistene.

2nd-1st centuries B.C.

OPEN FORMS (pls. 7-9, nos. 202-242) / Rim Fragments of a Bowl Form (pls. 7-8, nos. 202-220)

Incurved and rounded rim bowls are significant surface finds in Hellenistic southwestern Paphlagonia, as at all the major published Hellenistic sites in the Mediterranean. They are the most common and most widely distributed shape at Gordion (Stewart 2010, 195-196, figs. 218-222, nos. 226-281). Their size differs between medium $(12.8-14 \mathrm{~cm})$ and large $(14.2-30.8 \mathrm{~cm})$ dimensions. Typologically two subgroups of these bowls have been identifed: closed rim bowls and simple rim bowls. These bowls would have functioned as accommodating a variety of foods including soups, stews, and mashes, as well as side dishes of fruits and nuts (Stewart 2010, 196). They have a thin slip; in their interior and exterior faces wheelmarks are very distinctive.

202. (No. 1094): Rim fragment; Kepez, found in 2005. pl. 7/202 and pl. 35/202.

Max. h $2.2 \mathrm{~cm}$., max. w $2.5 \mathrm{~cm}$., max. th 0.5 $\mathrm{cm}$.

Very pale brown (10YR 7/4) unslipped surface on exterior and interior. Hard, thin paste, very sparsely porous, fine, reddish yellow
(7.5YR 6/6) fabric with frequent tiny lime inclusions.

203. (No. 1079): Rim fragment; Kepez, found in 2005. pl. 7/203 and pl. 35/203.

Max. h $2.5 \mathrm{~cm}$., max. w $3.8 \mathrm{~cm}$., max. th 0.5 $\mathrm{cm}$.

Very pale brown (10YR 8/3) slip on exterior; very pale brown (10YR 7/4) slip on interior. All of surface is burnished. Average hardness; thin paste, very sparsely porous, fine, reddish yellow (5YR 6/6) fabric with frequent tiny lime and some sand inclusions.

204. (No. 1085): Rim fragment; Kepez, found in 2005. pl. 7/204 and pl. 35/204.

Max. h $2.6 \mathrm{~cm}$., max. w $2.5 \mathrm{~cm}$., max. th 0.6 $\mathrm{cm}$.

Light grey (10YR 7/2) slip on exterior, pale brown (10YR 6/3) slip on interior rim. Exterior surface and interior rim are burnished. Hard, thin paste, non-porous, fired to yellowish red (5YR 5/6) and dark greyish brown (10YR 4/2) fabric with some micaceous inclusions.

205. (No. 1218): Rim fragment; Kimistene, summit of the Acropolis, southern slope, found in 2005. pl. 35/205.

Max. h $3.2 \mathrm{~cm}$., max. w $5.0 \mathrm{~cm}$., max. th 0.7 $\mathrm{cm}$.

Very pale brown (10YR 7/3) slip on exterior; very pale brown (10YR 7/4) slip on interior. All of surface is burnished. Average hardness; thin paste, very sparsely porous, fine, pale brown (10YR 6/3) fabric with infrequent sand inclusions.

206. (No. 991): Rim fragment; Kepez, found in 2005. pl. 7/206 and pl. 35/206.

Max. h $3.2 \mathrm{~cm}$., max. w $5.0 \mathrm{~cm}$., max. th 1.6 $\mathrm{cm}$.

Pink (7.5YR 7/4) slip on exterior and interior; 
light red (2.5YR 6/6) on the rim. Interior surface is burnished. Hard, very sparsely porous, fine, reddish yellow (7.5YR 7/6) fabric with frequent tiny lime and large grit inclusions.

207. (No. 1180): Rim fragment; Kepez, surface find in the Cistern, found in 2005.pl. 7/207 and pl. 35/207.

Max. h 2.4 cm., d of rim $12.8 \mathrm{~cm}$. , max. w 3.0 cm., max. th $0.5 \mathrm{~cm}$.

Very pale brown (10YR 7/3) slip on exterior; very pale brown (10YR 7/4) slip on interior. Average hardness; thin paste, non-porous, fine, very pale brown (10YR 7/3) fabric with some tiny lime inclusions.

208. (No. 1224): Rim fragment; Kimistene, summit of the Acropolis, southern slope, found in 2005. pl. 7/208 and pl. 35/208.

Max. h $2.8 \mathrm{~cm}$., d of rim $13.6 \mathrm{~cm}$., max. w 7.2 cm., max. th $0.5 \mathrm{~cm}$.

Reddish yellow (7.5YR 7/6) slip on exterior and interior. Exterior surface is burnished. Hard, thin paste, very sparsely porous, fine, pink (7.5YR 7/4) fabric with no visible inclusions.

209. (No. 1220): Rim fragment; Kimistene, summit of the Acropolis, southern slope, found in 2005. pl. 7/209 and pl. 35/209.

Max. h 2.7 cm., d of rim 14.0 cm., max. w 6.6 cm., max. th $0.6 \mathrm{~cm}$.

Very pale brown (10YR 7/3) slip on exterior and on interior. As inclusion medium calsite on exterior. Hard, thin paste, non-porous, fine, pink (7.5YR 7/4) fabric with occasio nal tiny lime inclusions.

210. (No. 692): Rim fragment; Kimistene, Acropolis, southern slope, just below the summit, up to Deresemail creek, found in 2005. pl. 7/210 and pl. 35/210.

Max. h 3.0 cm., d of rim 14.2 cm., max. w 4.4 cm., max. th $0.6 \mathrm{~cm}$.

Reddish yellow (7.5YR 6/6) slip on exterior, reddish yellow (7.5YR 7/6) slip on interior. All of surface is burnished. Hard, thin paste, non-porous, fine, yellowish red (5YR 5/6) fabric with rare sand and lime inclusions.

211. (No. 1078): Rim fragment; Kepez, found in 2005. pl. 7/211 and pl. 35/211.

Max. h 2.4 cm., d of rim $15.0 \mathrm{~cm}$., max. w 5.8 cm., max. th $0.5 \mathrm{~cm}$.

Very pale brown (10YR 7/4) unslipped surface on exterior; pink (5YR 7/4) unslipped surface on interior. Hard, thin paste, very sparsely porous, fine, reddish yellow (7.5YR 6/6) fabric with frequent lime and rare sand inclusions.

212. (No. 1077): Rim fragment; Kepez, found in 2005. pl. 7/212 and pl. 35/212.

Max. h 3.4 cm., d of rim $30.8 \mathrm{~cm}$., max. w 6.6 cm., max. th $1.2 \mathrm{~cm}$.

Very pale brown (10YR 7/3) slip on exterior; light yellowish brown (10YR 6/4) unslipped surface on interior. Average hardness; nonporous, fine, light brown (7.5YR 6/4) fabric with some medium grit and lime inclusions.

213. (No. 1068): Rim fragment; Kepez, found in 2005. pl. 7/213 and pl. 35/213.

Max. h $3.8 \mathrm{~cm}$., d of rim $16.2 \mathrm{~cm}$., max. w 9.3 cm., max. th $0.7 \mathrm{~cm}$.

Very pale brown (10YR 8/2) slip on the exterior and interior of the rim. Very pale brown (10YR 7/4) unslipped surface on lower interior. Exterior surface is burnished. Average hardness; thin paste, sparsely porous, fine, reddish yellow (7.5YR 6/6) fabric with frequent lime and rare small grit inclusion.

214. (No. 1070): Rim fragment; Kepez, found in 2005. pl. 7/214 and pl. 35/214.

Max. h $3.1 \mathrm{~cm}$., d of rim $17.0 \mathrm{~cm}$., max. w 3.8 
cm., max. th $0.7 \mathrm{~cm}$.

Thick wheel-marks. Exterior surface is burnished. Pinkish white (7.5YR 8/2) slip on exterior. Reddish yellow (7.5YR 7/6) unslipped surface on interior. Hard, thin paste, nonporous, fine, reddish yellow (5YR 7/6) fabric with frequent micaceous and some sand inclusion.

215. (No. 1221): Rim fragment; Kimistene, summit of the Acropolis, southern slope, found in 2005. pl. 7/215 and pl. 35/215.

Max. h 3.9 cm., d of rim $17.6 \mathrm{~cm}$., max. w 5.8 cm., max. th $0.6 \mathrm{~cm}$.

Reddish yellow (7.5YR 7/6) slip on exterior rim and interior. Reddish yellow (7.5YR 7/6) unslipped surface on lower exterior. Exterior rim and interior surface is burnished. Hard, thin paste, very sparsely porous, fine, reddish yellow (7.5YR 6/6) fabric with some sand inclusions.

216. (No. 399): Rim fragment; Kimistene, Acropolis, below the Temple's terrace, eastern slope, found in 2005. pl. 8/216 and pl. 35/216.

Max. h 2.4 cm., d of rim 18.4 cm., max. w 3.6 cm., max. th $0.9 \mathrm{~cm}$.

Very pale brown (10YR 7/4) slip on exterior; reddish yellow (7.5YR 6/6) slip on interior. Interior surface is burnished. Hard, thin paste, non-porous, fine, reddish yellow (5YR 6/6) fabric with frequent lime and sand, rare micaceous inclusions.

217. (No. 1082): Rim fragment; Kepez, found in 2005. pl. 8/217 and pl. 35/217.

Max. h $2.5 \mathrm{~cm}$., d of rim $19.0 \mathrm{~cm}$., max. w 4.1 cm., max. th $0.7 \mathrm{~cm}$.

Very pale brown (10YR 8/2) on exterior and interior rim. Very pale brown (10YR 7/4) unslipped surface on lower interior. Hard, thin paste, non-porous, fine, reddish yellow
(7.5YR 7/6) fabric with some tiny lime inclusions.

218. (No. 1277): Rim fragment; Kimistene, Necropolis, found in 2005. pl. 8/218 and pl. 35/218.

Max. h $2.6 \mathrm{~cm}$., d of rim $21.0 \mathrm{~cm}$., max. w 5.2 cm., max. th $0.6 \mathrm{~cm}$.

Reddish yellow (7.5YR 6/6) slip on exterior; pink (7.5YR 7/4) unslipped surface on interior. Exterior surface is burnished. Hard, thin paste, non-porous, fired to reddish yellow (7.5YR 6/6-5YR 6/8) fabric with rare grog, sand and tiny lime inclusions.

219. (No. 1394): Rim fragment; Kimistene, summit of the Acropolis, surface find, found in 2005. pl. 8/219 and pl. 36/219.

Max. h $5.2 \mathrm{~cm}$., d of rim $25.0 \mathrm{~cm}$., max. w 7.6 cm., max. th $0.8 \mathrm{~cm}$.

Reddish yellow (7.5YR 6/6) slip on exterior; light brown (7.5YR 6/3) unslipped surface on interior. Exterior surface is burnished. Hard, non-porous, fired to light brown (7.5YR 6/4) fabric with frequent tiny lime inclusions.

220. (No. 1214): Rim fragment; Kimistene, summit of the Acropolis, southern slope, found in 2005. pl. 8/220 and pl. 36/220.

Max. h $6.8 \mathrm{~cm}$., d of rim $25.2 \mathrm{~cm}$., max. w 4.6 cm., max. th $1.1 \mathrm{~cm}$.

Light yellowish brown (10YR 6/4) slip on exterior and interior. Exterior surface is burnished. Hard, non-porous, fine, reddish brown (5YR 5/4) fabric with infrequent lime and small grit inclusions.

Other Rim Fragments (pl. 8, nos. 222228)

Some metal imitated shapes, such as carinated and ledge rim bowls are popular. Most of them are polished. In some other excavated sites 
these typologies were classified as "Achaemenid". However, in southwestern Paphlagonia these vessel forms cannot be assigned to any known group.

221. (No. 801): Rim fragment; Kimistene, summit of the Acropolis, western slope, found in 2005. pl. 36/221.

Max. h $2.8 \mathrm{~cm}$., max. w $2.2 \mathrm{~cm}$., max. th 0.7 $\mathrm{cm}$.

Light yellowish brown (10YR 6/4) slip on exterior; pale brown (10YR 6/3) slip on interior. All of surface is burnished. Hard, non-porous, fine, pale brown (10YR 6/3) fabric with frequent sand and some small grit inclusions.

222. (No. 500): Rim fragment; Kimistene, Acropolis, southern slope, just below the summit, up to Deresemail creek, found in 2005. pl. 8/222 and pl. 36/222.

Max. h 1.8 cm., max. w $3.1 \mathrm{~cm}$., max. th 0.7 $\mathrm{cm}$.

Light brown (7.5YR 6/4) slip on exterior and interior. All of surface is burnished. Hard, non-porous, fine, brown (7.5YR 5/4) fabric with some sand inclusions.

223. (No. 862): Rim fragment; Kimistene, Acropolis, temple slope, illegally excavated pit, altar with boukronion, found in 2005. pl. 8/223 and pl. 36/223.

Max. h 2.0 cm., max. w 2.5 cm., max. th 0.7 $\mathrm{cm}$.

Pink (5YR 7/4) slip on exterior and interior. All of surface is burnished. Soft, non-porous, fine, reddish yellow (5YR 6/6) fabric with frequent sand inclusions.

Parallels: Sagona/Sagona 2004, fig. 159, 10 (from Çimentepe, 800-300 B.C.) and Şenyurt/ Kamış/Akçay 2005, 101, 141, type 4.4, fig. 9/108.

224. (No. 1299): Rim fragment; Kimistene, Acropolis, temple's terrace, illegally excavat- ed area under the temple's podium, found in 2005. pl. 36/224.

Max. h $2.1 \mathrm{~cm}$., max. w $2.7 \mathrm{~cm}$., max. th 0.9 $\mathrm{cm}$.

Light yellowish brown (10YR 6/4) slip on exterior and interior. All of surface is burnished. Hard, non-porous, fired to pale brown (10YR $6 / 3$ ) and black (2.5Y 2.5/1) fabric with frequent tiny lime inclusions.

225. (No. 955): Rim fragment; Kimistene, Acropolis, found in 2005. pl. 8/225 and pl. 36/225.

Max. h 2.3 cm., max. w 4.0 cm., max. th 1.0 $\mathrm{cm}$.

Light brown (7.5YR 6/4) slip on exterior; pink (7.5YR 7/4) slip on interior. Exterior surface is burnished. Hard, non-porous, fired to light brown (7.5YR 6/4) and grey (7.5YR $6 / 1$ ) fabric with frequent tiny lime and some sand inclusions.

Parallels: Şenyurt/Kamış/Akçay 2005, 95, 136, type 2.2, fig. $4 / 46$.

226. (No. 517): Rim fragment; Kimistene, Acropolis, southern slope, just below the summit, up to Deresemail creek, found in 2005. pl. 8/226 and pl. 36/226.

Max. h $3.2 \mathrm{~cm}$., max. w $2.3 \mathrm{~cm}$., max. th 1.2 $\mathrm{cm}$.

Light brown (7.5YR 6/4) slip on exterior and interior. All of surface is burnished. Hard, non-porous, fine, brown (7.5YR 5/4) fabric with some sand inclusions.

227. (No. 1075): Rim fragment; Kepez, found in 2005. pl. 36/227.

Max. h $1.8 \mathrm{~cm}$., max. w $3.8 \mathrm{~cm}$., max. th 1.1 $\mathrm{cm}$.

Light brown (7.5YR 6/4) slip on exterior; pink (7.5YR 7/4) unslipped surface on interior. Exterior surface is burnished. Soft, very sparsely porous, fine, yellowish red (5YR 5/6) 
fabric with frequent tiny lime inclusions.

228. (No. 1457): Rim fragment; uncertain. pl. $8 / 228$ and pl. 36/228.

Max. h $2.2 \mathrm{~cm}$., max. w $4.4 \mathrm{~cm}$., max. th 0.7 $\mathrm{cm}$.

Reddish brown (5YR 5/4) slip on exterior and interior. All of surface is burnished. Hard, non-porous, fine, brown (10YR 5/3) fabric with occasional sand and grog inclusions.

Parallel: Şenyurt/Ekmen 2005, 62-63, 73, 113, fig. 66, type 1.17/8.

Base Fragments of Open Forms (pls. 8-9, nos. 230-233)

High-based profiles.

229. (No. 1165): Base fragment; Kepez, Cistern, found in 2005. pl. 36/229.

Max. h $2.8 \mathrm{~cm}$., max. w $7.5 \mathrm{~cm}$., max. th 0.8 $\mathrm{cm}$

Very pale brown (10YR 7/4) slip on exterior and interior. Average hardness; thin paste, very sparsely porous, fine, light brown (7.5YR 6/4) fabric with frequent tiny lime and rare small grit inclusions.

230. (No. 1158): Base fragment; Kepez, Cistern, found in 2005. pl. 8/230 and pl. 36/230. Max. h $3.7 \mathrm{~cm}$., d of base $6.4 \mathrm{~cm}$., max. w 11.5 cm., max. th $0.7 \mathrm{~cm}$.

Surface is smoothed on the exterior. A red (2.5YR 5/6) tiny slip on the exterior. Reddish yellow (5YR 6/6-7.5YR 7/6) unslipped surface on interior. Average hardness; sparsely porous, thin paste; fine, light red (2.5YR 6/8) fabric with frequent lime and some small grit inclusion.

231. (No. 988): Base fragment; Kepez, found in 2005. pl. 9/231 and pl. 36/231.

Max. h $2.8 \mathrm{~cm}$., d of base $6.8 \mathrm{~cm}$., max. w 6.9 cm., max. th $0.7 \mathrm{~cm}$.

Pink (7.5YR 7/4) slip on exterior; exterior surface thinly slipped. Reddish yellow (5YR 6/6) slip on interior. It is burnished on the middle of interior. Hard, non-porous, thin paste, fired to reddish yellow (5YR 6/6) fabric with no visible inclusions.

232. (No. 1322): Base fragment; Kimistene, summit of the Acropolis, southern slope, found in 2005. pl. 9/232 and pl. 36/232.

Max. h $2.8 \mathrm{~cm}$., d of base $7.4 \mathrm{~cm}$., max. w 4.9 cm., max. th $0.7 \mathrm{~cm}$.

Very pale brown (10YR 8/2) slip on exterior and interior. Interior surface is burnished. Hard, thin paste, non-porous, fired to reddish yellow (5YR 6/8) and pale brown (10YR 6/3) fabric with no visible inclusions.

233. (No. 466): Base fragment; Kimistene, Acropolis, southern slope, just below the summit, up to Deresemail creek, found in 2005. pl. 9/233 and pl. 36/233.

Max. h $2.4 \mathrm{~cm}$., d of base $7.6 \mathrm{~cm}$., max. w 5.7 cm., max. th $0.8 \mathrm{~cm}$.

Very pale brown (10YR 8/2) slip on exterior; pink (7.5YR 7/4) slip on interior. Interior surface is burnished. Hard, thin paste, nonporous, fine, reddish yellow (5YR 6/6) fabric with infrequent tiny lime inclusions.

Body Fragments of Open Forms (pl. 9, no. 242)

234. (No. 1193): Body fragment; Kepez, Cistern, found in 2005. pl. 36/234.

Max. h $1.5 \mathrm{~cm}$., max. w $1.6 \mathrm{~cm}$., max. th 0.5 $\mathrm{cm}$.

Reddish yellow (5YR 6/6) slip on exterior; reddish yellow (7.5YR 7/6) slip on interior. Hard, thin paste, non-porous, fine, reddish yellow (5YR 6/8) fabric with frequent micaceous, rare sand inclusions. 
235. (No. 1057): Body fragment; Kepez, found in 2005. pl. 36/235.

Max. h $2.3 \mathrm{~cm}$., max. w $2.6 \mathrm{~cm}$., max. th 0.5 $\mathrm{cm}$.

Pink (7.5YR 8/4) slip on exterior; reddish yellow (5YR 6/6) unslipped surface on interior. Hard, thin paste, non-porous, fine, reddish yellow (5YR 6/6) fabric with no visible inclusions.

236. (No. 1058): Body fragment; Kepez, found in 2005. pl. 36/236.

Max. h 2.5 cm., max. w $2.5 \mathrm{~cm}$., max. th 0.5 $\mathrm{cm}$.

Yellowish red (5YR 5/6) slip on upper exterior. Upper exterior is burnished. Its below part has pink (7.5YR 7/4) unslipped surface. Reddish yellow (5YR 6/6) slip on interior, hard, thin paste, non-porous, fine, light red (2.5YR 6/8) fabric with some tiny lime and rare sand inclusions.

237. (No. 1024): Body fragment; Kepez, found in 2005. pl. 37/237.

Max. h 2.6 cm., max. w 2.6 cm., max. th 0.6 $\mathrm{cm}$.

Very pale brown (10YR 7/3) slip on exterior; very pale brown (10YR 7/4) thin slip on interior. All of surface is burnished. Hard, thin paste, non-porous, fine, yellowish red (5YR 5/6) fabric with rare sand inclusions.

238. (No. 841): Body fragment; Kimistene, Cistern, eastern slope, found in 2005. pl. 37/238.

Max. h $2.3 \mathrm{~cm}$., max. w 2.9 cm., max. th 0.6 cm.

Pink (7.5YR 7/3) slip on exterior; pink (7.5YR 7/4) slip on interior. Hard, thin paste, non-porous, fired to reddish yellow (7.5YR 6/6) and brown (7.5YR 5/2) mottled fabric with no visible inclusions.
239. (No. 1036): Body fragment; Kepez, found in 2005. pl. 37/239.

Max. h 2.7 cm., max. w 2.3 cm., max. th 0.7 $\mathrm{cm}$.

Very pale brown (10YR 7/4) thin slip on exterior and interior. Average hardness; thin paste, non-porous, fine, light brown (7.5YR 6/4) fabric with rare sand inclusions.

240. (No. 759): Body fragment; Kimistene, Acropolis, temple's terrace, illegally excavated area under the temple's podium, found in 2005. pl. 37/240.

Max. h $3.5 \mathrm{~cm}$., max. w $2.4 \mathrm{~cm}$., max. th 0.7 cm.

Pink (7.5YR 8/4) slip on exterior, pink (7.5YR 7/4) slip on interior. Average hardness; thin paste, non-porous, fine, light brown (7.5YR $6 / 4$ ) fabric with frequent tiny lime inclusions.

241. (No. 1028): Body fragment; Kepez, found in 2005. pl. 37/241.

Max. h $2.5 \mathrm{~cm}$., max. w $3.3 \mathrm{~cm}$., max. th 0.7 $\mathrm{cm}$.

Very pale brown (10YR 8/3) slip on exterior and interior. All of surface is burnished. Hard, thin paste, non-porous, fine, light brown (7.5YR 6/4) fabric with no visible inclusions.

242. (No. 814): Body fragment; Kimistene, summit of the Acropolis, western slope, found in 2005. pl. 9/242 and pl. 37/242.

Max. h $3.2 \mathrm{~cm}$., max. w $4.0 \mathrm{~cm}$., max. th 0.5 $\mathrm{cm}$

Reddish yellow (5YR 7/6) unslipped surface on exterior, very pale brown (10YR 7/4) unslipped surface on interior. Hard, thin paste, non-porous, fine, light brown (7.5YR 6/4) fabric with some sand inclusions.

243. (No. 1160): Body fragment; Kepez, surface find in the Cistern, found in 2005. pl. $37 / 243$. 
Max. h 1.9 cm., max. w $4.3 \mathrm{~cm}$., max. th 1.0 $\mathrm{cm}$.

Reddish yellow (7.5YR 6/6) slip on exterior. Its upper part is reddish yellow (7.5YR 7/6) unslipped surface on exterior. Reddish yellow (7.5YR 7/6) slip on interior. The connection area of base and belly is burnished and slipped on the exterior. Average hardness; thin paste, very sparsely porous, fine, yellowish red (5YR 5/6) fabric with frequent tiny lime inclusions.

244. (No. 436): Body fragment; Kimistene, Acropolis, slope, found in 2005. pl. 37/244.

Max. h 1.3 cm., max. w $4.6 \mathrm{~cm}$., max. th 0.7 $\mathrm{cm}$.

Pale brown (10YR 6/3) slip on exterior and interior. All of surface is burnished. Average hardness; thin paste, non-porous, fired to brown (10YR 5/3) and greyish brown (10YR $5 / 2$ ) fabric with no visible inclusions.

245. (No. 995): Body fragment; Kepez, found in 2005. pl. 37/245.

Max. h 3.9 cm., max. w $4.5 \mathrm{~cm}$., max. th 0.6 $\mathrm{cm}$.

Light grey (10YR 7/2) thin slip on exterior and interior. Hard, thin paste, non-porous, fired to greyish brown (10YR 5/2) fabric with no visible inclusions.

246. (No. 1013): Body fragment; Kepez, found in 2005. pl. 37/246.

Max. h $2.9 \mathrm{~cm}$., max. w $5.7 \mathrm{~cm}$., max. th 0.5 $\mathrm{cm}$.

Very pale brown (10YR 8/4) slip on exterior; reddish yellow (5YR 6/6) slip on interior. Interior surface is burnished. Hard, thin paste, non-porous, fine, reddish yellow (7.5YR 6/6) fabric with no visible inclusions.

247. (No. 1173): Body fragment; Kepez, Cistern, found in 2005. pl. 37/247.
Max. h 3.4 cm., max. w $4.3 \mathrm{~cm}$., max. th 0.7 $\mathrm{cm}$.

Very pale brown (10YR 7/3) slip on exterior and interior. Exterior surface is burnished. Average hardness; thin paste, very sparsely porous, fine, pale brown (10YR 6/3) fabric with some micaceous inclusions.

248. (No. 1166): Body fragment; Kepez, Cistern, found in 2005. pl. 37/248.

Max. h $4.0 \mathrm{~cm}$., max. w $6.8 \mathrm{~cm}$., max. th 0.7 $\mathrm{cm}$.

Very pale brown (10YR 7/4) thin slip on exterior and interior. Hard, thin paste, non-porous, fine, light brown (7.5YR 6/4) fabric with occasional tiny lime and sand inclusions.

249. (No. 1168): Body fragment; Kepez, Cistern, found in 2005. pl. 37/249.

Max. h $4.3 \mathrm{~cm}$., max. w $5.7 \mathrm{~cm}$., max. th 0.5 $\mathrm{cm}$.

Very pale brown (10YR 7/4) slip on exterior and interior. Exterior surface is burnished. Hard, thin paste, non-porous, fine, pale brown (10YR 6/3) fabric with some sand inclusions.

CLOSED FORMS (pl. 9, nos. 250-256) / A Body Fragment of a Rhyton (pl. 9, no. 250)

250. (No. 1170): Body fragment; Kepez, Cistern, found in 2005. pl. 9/250 and pl. 37/250. Max. h 6.4 cm., max. w $4.1 \mathrm{~cm}$., max. th 0.8 $\mathrm{cm}$.

Very pale brown (10YR 7/3) slip on exterior; very pale brown (10YR 7/4) unslipped surface on interior. Engraved linear decoration on the exterior. Soft, thin paste, non-porous, fine, pale brown (10YR 6/3) fabric with occasional tiny lime and frequent sand inclusions. 
Base Fragments of Closed Form (pl. 9, nos. 251-255)

High-based forms. Some of them were polished.

251. (No. 1197): Base fragment; Kepez, Cistern, found in 2005. pl. 9/251 and pl. 37/251. Max. h 1.1 cm., max. w 2.0 cm., max. th 0.7 $\mathrm{cm}$.

Pink (7.5YR 8/4) unslipped surface on exterior and interior. Hard, thin paste, very sparsely porous, fine, reddish yellow (5YR 6/6) fabric with some sand and rare tiny lime inclusions.

252. (No. 1396): Base fragment; Kimistene, summit of the Acropolis, found in 2005. pl. 9/252 and pl. 37/252.

Max. h 1.7 cm., d of rim 6.4 cm., max. w 3.3 cm., max. th $0.7 \mathrm{~cm}$.

Reddish yellow (5YR 6/6) slip on exterior; reddish yellow (5YR 7/6) unslipped surface on interior. Exterior surface is burnished. Hard, thin paste, sparsely porous, fired to yellowish red (5YR 5/6) and reddish yellow (7.5YR 6/6) mottled fabric with rare small grit inclusions.

253. (No. 1109): Base fragment; Kepez, Cistern, found in 2005. pl. 9/253 and pl. 37/253. Max. h 1.0 cm., d of rim 9.4 cm., max. w 3.3 cm., max. th $0.6 \mathrm{~cm}$.

Very pale brown (10YR 7/3) slip on exterior; very pale brown (10YR 7/4) slip on interior. Hard, thin paste, very sparsely porous, fine, light brown (7.5YR 6/4) fabric with no visible inclusions.

254. (No. 503): Base fragment; Kimistene, Acropolis, southern slope, just below the summit, up to Deresemail creek, found in 2005. pl. 9/254 and pl. 37/254.

Max. h 2.6 cm., d of rim 11.6 cm., max. w 7.5 cm., max. th $1.0 \mathrm{~cm}$.

Pink (7.5YR 7/4) slip on exterior and interior. Exterior surface is burnished. Hard, thin paste, very sparsely porous, fired to brown (7.5YR 5/4) fabric with some tiny lime and sand inclusions.

255. (No. 665): Base fragment; Kimistene, Cistern, eastern slope, found in 2005. pl. 9/255 and pl. 37/255.

Max. h 2.4 cm., d of rim $12.0 \mathrm{~cm}$., max. w 6.6 cm., max. th $0.8 \mathrm{~cm}$.

Reddish yellow (7.5YR 7/6) slip on exterior. Reddish yellow (5YR 6/6) unslipped surface on the exterior of connection area between base and belly. Reddish yellow (7.5YR 7/6) unslipped surface on interior. The area between base and belly is also burnished. Hard, thin paste, sparsely porous, fine, reddish yellow (7.5YR 6/6) fabric with rare lime, infrequent sand inclusions.

Handle Fragment of Closed Form (pl. 9, nos. 256)

Exterior is burnished.

256. (No. 709): Handle fragment; Kimistene, Acropolis, temple slope, eastern slope, surface find, found in 2005. pl. 9/256 and pl. 37/256. Max. h $3.5 \mathrm{~cm}$., max. w $4.5 \mathrm{~cm}$., max. th 1.6 $\mathrm{cm}$.

Very pale brown (10YR 7/4) slip on exterior; very pale brown (10YR 7/3) unslipped surface on interior. Exterior surface is burnished. Hard, thin paste, non-porous, fine, very pale brown (10YR 7/4) fabric with frequent tiny lime inclusions.

Body Fragments of Closed Forms (pl. 37, nos. 257-261)

All of them have a light slip. 
257. (No. 1236): Body fragment; Kepez, found in 2005. pl. 37/257.

Max. h $2.0 \mathrm{~cm}$., max. w $3.3 \mathrm{~cm}$., max. th 0.6 $\mathrm{cm}$.

Reddish yellow (5YR 6/6) slip on exterior; red (2.5YR 5/6) unslipped surface on interior. Hard, thin paste, non-porous, fired to red (2.5YR 5/8) and light brown (7.5YR 6/3) fabric with frequent tiny lime and occasional sand inclusions.

258. (No. 1181): Body fragment; Kepez, Cistern, found in 2005. pl. 37/258.

Max. h $2.2 \mathrm{~cm}$., max. w $4.5 \mathrm{~cm}$., max. th 0.6 $\mathrm{cm}$.

Very pale brown (10YR 8/3) slip on exterior; reddish yellow (5YR 7/6) unslipped surface on interior. Average hardness; thin paste, nonporous, fine, reddish yellow (7.5YR 6/6) fabric with frequent tiny lime and rare small grit inclusions.

259. (No. 1232): Body fragment; Kepez, found in 2005. pl. 37/259.

Max. h $1.3 \mathrm{~cm}$., max. w $6.2 \mathrm{~cm}$., max. th 1.1 $\mathrm{cm}$.

Reddish yellow (7.5YR 6/6) slip on exterior. Reddish yellow (5YR 6/6) unslipped surface on interior. Exterior surface is burnished. Average hardness; thin paste, very sparsely porous, fine, reddish yellow (5YR 6/6) fabric with rare micaceous and sand inclusions.

260. (No. 1172): Body fragment; Kepez, Cistern, found in 2005. pl. 37/260.

Max. h $5.5 \mathrm{~cm}$., max. w $4.0 \mathrm{~cm}$., max. th 0.6 $\mathrm{cm}$.

Very pale brown (10YR 7/4) slip on exterior and interior neck. The other part has very pale brown (10YR 7/4) unslipped surface on interior. Exterior surface is burnished. Hard, thin paste, non-porous, fine, very pale brown (10YR 7/4) fabric with some sand inclusions.
261. (No. 1161): Body fragment; Kepez, surface find in the Cistern, found in 2005. pl. $37 / 261$.

Max. h $5.0 \mathrm{~cm}$., max. w $5.6 \mathrm{~cm}$., max. th 0.9 $\mathrm{cm}$.

Very pale brown (10YR 8/3) slip on exterior; reddish yellow (5YR 6/6) unslipped surface on interior. Average hardness; thin paste, nonporous, fine, light yellowish brown (10YR 6/4) fabric with infrequent sand, some tiny lime inclusions.

IX. RED-PAINTED KEPEZ GROUP (pls. 10-16, nos. 262-395)

There should be numerous workshops of Hellenistic local painted pottery in northern and central Asia Minor, such as the one discovered at Oluz Höyük. Our Kepez group, most of which consists of small sherds is a further one. They have frequently one or two red or brownish bands in the interior and thin bands around the rim. Especially on the shoulders and interior faces this ornamentation has been applied by means of a compass.

The most common form is that of bowls that are partially slipped. Some of them are incurved rim bowls (cf. nos. 279 and 292) with a thickness of 0.4-1.0 cm. Fish plates with downturned rims (nos. 308 and 313) are also attested. No. 274 is an hemispherical form with two grooves on the upper exterior of the rim. Most of the sherds are of bases and rims. The major form is the hemispherical bowl with thin walls and smoothed surface.

Paste is reddish yellow (5YR 6/6-6/8-7/67/8, 7.5YR 6/6-7/6), light red (2.5YR 6/8-6/6), light brown (7.5YR 6/3-6/4), red (10R 5/8, 2.5YR 5/6-5/8), pink (5 YR 7/4, 7.5YR 7/3-7/4), yellowish red (5YR 5/6), very pale brown (10YR 7/3$7 / 4$ ), reddish brown (5YR 4/4-5/4), brown (7.5YR 4/4-5/3-5/4), light reddish brown (5YR 6/4) and light yellowish brown (10YR 6/4). Hard paste with lime, sand, grit, micaceous, grog and quartz 
inclusions in small dimensions. In some examples there is no inclusion. The slip is red $(10 \mathrm{R}$ 4/6-5/6-5/8, 2.5YR 4/6-4/8-5/6-5/8), reddish yellow (5YR 6/6-6/8-7/6, 7.5YR 6/6-7/6), very pale brown (10YR 8/2-8.5/2-8/3-8/4-7/3-7/4), pink (5YR 7/4-8/3, 7.5YR 7/4-8/3), light red (2.5YR 6/6-6/8), reddish brown $(2.5 \mathrm{YR} 4 / 4-5 / 4$, 5YR 4/3-5/4), weak red (10R 4/4-5/4), brown (7.5YR 4/2-4/3-5/4, 10YR 5/3), light brown (7.5YR 6/4), yellowish red (5YR 5/6), light grey (10YR 7/2) and yellow (10YR 7/6). Due to the changing fire conditions or brush use the slip colors change to be mottled. It is non-porous.

The elaborate method of decoration was primarily slip, applied in bands. These decorative bands with $0.7-2.0 \mathrm{~cm}$ were applied in red (10R 5/8-5/6, 2.5YR 4/6-5/6-5/8), pinkish white (7.5YR 8/2, 5YR 8/2), weak red (7.5R 4/4, 10R 5/4-4/2, 2.5YR 4/2), light red (2.5YR 6/6-6/8), reddish brown (2.5YR 5/4-4/3, 5YR 5/4-4/4-4/3), reddish yellow (5YR 6/6, 7.5YR 7/6-7/8-6/6), dusky red (2.5YR $3 / 2)$, very dark grey (7.5YR $3 / 1$ ), very pale brown (10YR 8/2), black (5YR 2.5/1, 7.5YR 2.5/1), light brown (7.5YR 6/4), yellowish red (5YR 5/6), dark greyish brown (10YR 4/2), light reddish brown (5YR 6/4), dark reddish grey (2.5YR 3/1) and white (5YR 8/1). Three different painting methods have been applied.

134 sherds were collected in total. 112 fragments are open forms and 22 closed forms.

90 of them were found at Kepez and 30 of them were from the southern slope of the Acropolis at Kimistene. In any case this is a local group, assigned to southwestern Paphlagonia.

2nd-1st centuries B.C.

OPEN FORMS (pl. 10-15, nos. 262-373) /

Bowl Form 1 (pls.10-11, nos. 262-284)

This bowl type is an incurved rim bowl which is a small open vessel with a deep interior with the maximum diameter near the upper quar- ter of the wall and a ring or false ring foot. Most of them were decorated with red and red-brown bands. Their rim parts were painted with brush in red. These thin bands continue also in the vessels itself. Some of them do not have any slip on their bottom part. Their surfaces are dull. Their dimensions differ between 12.0 and $27.2 \mathrm{~cm}$. In some samples there are two grooves on the upper exterior just below the rim.

262. (No. 586): Rim fragment; Kimistene, Acropolis, southern slope, just below the summit, up to Deresemail creek, found in 2005. pl. 10/262 and pl. 38/262.

Max. h $1.2 \mathrm{~cm}$., w $1.8 \mathrm{~cm}$., max. th $0.3 \mathrm{~cm}$.

Red (10R 5/8) on exterior and interior rims; their below parts have light red (2.5YR 6/6) unslipped surface. Hard, non-porous, thin paste, fine, red (2.5YR 5/6) fabric with some micaceous inclusions.

263. (No. 1105): Rim fragment; Kepez, found in 2005. pl. 10/263 and pl. 38/263.

Max. h $1.7 \mathrm{~cm}$., max. w $1.7 \mathrm{~cm}$., max. th 0.5 $\mathrm{cm}$.

Red (2.5YR 5/6) slip on exterior and interior. Average hardness; very sparsely porous, thin paste, non-porous, fine, reddish yellow (5YR 7/8) fabric with rare micaceous and lime inclusions.

264. (No. 1062): Rim fragment; Kepez, found in 2005. pl. 10/264 and pl. 38/264.

Max. h 1.9 cm., max. w $1.7 \mathrm{~cm}$., max. th 0.5 $\mathrm{cm}$.

Weak red (10R 5/4) on exterior rim; its below part has pink (7.5YR 7/4) unslipped surface on exterior. Reddish yellow (7.5YR 7/6) slip on interior. Hard, thin paste, non-porous, fine, reddish yellow (7.5YR 7/6) fabric with no visible inclusions.

265. (No. 1086): Rim fragment; Kepez, found 
in 2005. pl. 10/265 and pl. 38/265.

Max. h $2.1 \mathrm{~cm}$., max. w $2.3 \mathrm{~cm}$., max. th 0.6 $\mathrm{cm}$.

Light red (2.5YR 6/6) on exterior rim; its below part has pink (7.5YR 7/4) unslipped surface. Red (2.5YR 5/6) on interior. Average hardness; non-porous, thin paste, fine, reddish yellow (5YR 6/6) fabric with some sand, lime and micaceous inclusions.

266. (No. 885): Rim fragment; Kimistene, Acropolis, southern slope, just below the summit, up to Deresemail creek, found in 2005. pl. 10/266 and pl. 38/266.

Max. h 2.4 cm., max. w $2.0 \mathrm{~cm}$., max. th 0.5 $\mathrm{cm}$.

Yellowish red (5YR 5/6) on exterior rim; its below part has very pale brown (10YR 7/4) on unslipped surface. Light red (2.5YR 6/6) on interior rim; its below part has pink (7.5YR 7/3) on unslipped surface. Hard, non-porous, thin paste, fired to light brown (7.5YR 6/3) and grey (10YR 5/1) fabric with some tiny lime inclusions.

267. (No. 953): Rim fragment; Kimistene, Acropolis, found in 2005. pl. 10/267 and pl. 38/267.

Max. h 2.9 cm., max. w $2.6 \mathrm{~cm}$., max. th 0.6 $\mathrm{cm}$.

Reddish brown (2.5YR 5/4) on exterior rim and interior rim. Their below parts have pink (7.5YR 7/4) unslipped surface. Two shallow groove enclose all of exterior rim. Hard, thin paste, non-porous, fine, reddish yellow (7.5YR 6/6) fabric with rare lime inclusions.

268. (No. 1190): Rim fragment; Kepez, Cistern, found in 2005. pl. 10/268 and pl. 38/268. Max. h $2.3 \mathrm{~cm}$., max. w $2.7 \mathrm{~cm}$., max. th 0.7 $\mathrm{cm}$.

Reddish brown (2.5YR 4/3) on exterior rim; its below part has reddish yellow (5YR 7/6) unslipped on exterior. Light brown (7.5YR 6/4) slip on interior. It is burnished on interior surface. Hard, thin paste, sparsely porous, fine, yellowish red (5YR 5/6) fabric with some lime inclusions.

269. (No. 802): Rim fragment; Kimistene, summit of the Acropolis, western slope, found in 2005. pl. 10/269 and pl. 38/269.

Max. h 2.4 cm., max. w $3.1 \mathrm{~cm}$., max. th 0.6 $\mathrm{cm}$.

Light red (2.5YR 6/6) on exterior rim and interior. Lower exterior has pink (7.5YR 7/4) unslipped surface. This part is burnished. Hard, non-porous, thin paste, fine, reddish brown (5YR 5/4) fabric with occasional tiny lime and sand inclusions.

270. (No. 1093): Rim fragment; Kepez, found in 2005. pl. 10/270 and pl. 38/270.

Max. h $2.2 \mathrm{~cm}$., max. w $3.1 \mathrm{~cm}$., max. th 0.5 $\mathrm{cm}$.

Reddish yellow (5YR 6/6) on exterior and interior rim. Very pale brown (10YR 7/4) slip on lower interior. Reddish brown (5YR 4/3) band on middle of the interior. Hard, thin paste, non-porous, fine, brown (7.5YR 5/4) fabric with infrequent sand inclusions.

271. (No. 645): Rim fragment; Kimistene, Acropolis, western slope, found in 2005. pl. 10/271 and pl. 38/271.

Max. h $3.5 \mathrm{~cm}$., max. w $4.1 \mathrm{~cm}$., max. th 0.9 $\mathrm{cm}$.

Red (2.5YR 5/8) on exterior rim and interior rim. Their below parts have reddish yellow (5YR 6/6) unslipped surface. Exterior surface is burnished. Hard, thin paste, very sparsely porous, fine, light red (2.5YR 6/8) fabric with frequent tiny lime inclusions.

272. (No. 966): Rim fragment; Kimistene, the surface find of the Cistern, eastern slope, 
found in 2005. pl. 10/272 and pl. 38/272.

Max. h 4.2 cm., max. w $4.6 \mathrm{~cm}$., max. th 0.6 $\mathrm{cm}$.

Red (2.5YR 5/6) band on exterior rim and interior rim; their below parts have reddish yellow (7.5YR 7/6) slip all of surface. Interior surface is burnished. Average hardness; sparsely porous, thin paste, fine, reddish yellow (7.5YR 7/6) fabric with no visible inclusions.

273. (No. 999): Rim fragment; Kepez, found in 2005. pl. 38/273.

Max. h 4.6 cm., max. w 4.5 cm., max. th 1.0 $\mathrm{cm}$.

Red (2.5YR 5/6) band on exterior rim and interior rim. Their below parts have reddish yellow (5YR 6/6) unslipped surface. Average hardness; very sparsely porous, fine, reddish yellow (5YR 6/6) fabric with some small lime and small grit inclusions.

274. (No. 1071): Rim fragment; Kepez, found in 2005. pl. 10/274 and pl. 38/274.

Max. h $4.1 \mathrm{~cm}$., d of rim $12.0 \mathrm{~cm}$., max. w 5.2 $\mathrm{cm}$., max. th $0.6 \mathrm{~cm}$.

Two shallow grooves enclose all of the exterior rim; reddish brown (2.5YR 5/4) slip on exterior rim. Its lower part has a very pale brown (10YR 7/4) unslipped interior and exterior surface. Hard, non-porous, fine, reddish yellow (7.5YR 6/6) fabric with infrequent sand inclusions and with traces of mica.

275. (No. 852): Rim fragment; Kimistene, found in 2005. pl. 10/275 and pl. 38/275.

Max. h 2.1 cm., d of rim 12.8 cm., max. w 2.4 cm., max. th $0.5 \mathrm{~cm}$.

Red (2.5YR 5/6) abrasion slip on exterior and interior. Hard, thin paste, non-porous, fine, pink (7.5YR 7/4) fabric with no visible inclusions.
276. (No. 1227): Rim fragment; Kimistene, summit of the Acropolis, southern slope, found in 2005. pl. 10/276 and pl. 38/276.

Max. h $4.0 \mathrm{~cm}$. , d of rim $13.2 \mathrm{~cm}$., max. w 4.1 cm., max. th $0.6 \mathrm{~cm}$.

Red (2.5YR 5/6) on exterior rim and interior rim. Their below parts have reddish yellow (7.5YR 7/6) thin slip, hard, non-porous, thin paste, fine, reddish yellow (7.5YR 6/6) fabric with occasional sand and micaceous, some tiny lime inclusions.

277. (No. 1204): Rim fragment; Kimistene, summit of the Acropolis, southern slope, found in 2005. pl. 10/277 and pl. 38/277.

Max. h 3.4 cm., d of rim $27.2 \mathrm{~cm}$., max. w 5.7 cm., max. th $0.8 \mathrm{~cm}$.

Reddish brown (5YR 5/4) on exterior rim; its below part has light brown (7.5YR 6/4) unslipped surface on exterior and interior rim, light brown (7.5YR 6/4) slip on lower interior. This part is burnished. Hard, thin paste, nonporous, fine, brown (7.5YR 5/4) fabric with frequent tiny lime, infrequent medium grit inclusions.

278. (No. 1217): Rim fragment; Kimistene, summit of the Acropolis, southern slope, found in 2005. pl. 10/278 and pl. 38/278.

Max. h $4.0 \mathrm{~cm}$., d of rim $14.0 \mathrm{~cm}$., max. w 4.1 cm., max. th $0.7 \mathrm{~cm}$.

Red (2.5YR 5/6) on exterior rim and interior. Its below part has very pale brown (10YR 7/3) slip on exterior. This part is burnished. Hard, thin paste, non-porous, fine, yellowish red (5YR 5/6) fabric with some tiny lime inclusions.

279. (No. 1095): Rim fragment of an incurved rim bowl; Kepez, found in 2005. pl. 11/279 and pl. 38/279.

Max. h $2.0 \mathrm{~cm}$., d of rim $14.2 \mathrm{~cm}$., max. w 6.9 cm., max. th $0.5 \mathrm{~cm}$. 
Reddish yellow (7.5YR 7/6) and brown (7.5YR 4/2) mottled slip on exterior; brown (10YR 5/3) and yellow (10YR 7/6) mottled slip on interior. Hard, thin paste, non-porous, fine, light yellowish brown (10YR 6/4) fabric with occasional sand and lime inclusions.

Parallels: Rotroff 1997, 341, fig. 63, no. 1002; and Stewart 2010, 281, fig. 102d and fig. 220, n. 259 (P 2841; from Gordion).

280. (No. 1412): Rim fragment; Kepez, surface find, found in 2005. pl. 11/280 and pl. 38/280.

Max. h $3.0 \mathrm{~cm}$., d of rim $15.2 \mathrm{~cm}$., max. w 2.8 cm., max. th $1.1 \mathrm{~cm}$.

Red (2.5YR 5/6) on exterior rim; its below part has pink (7.5YR 7/4) unslipped surface. Same color as the interior. Hard, porous, thin paste, fine, reddish yellow (5YR 6/6) fabric with some sand, frequent lime inclusions.

281. (No. 838): Rim fragment; Kimistene, Cistern, eastern slope, found in 2005. pl. 11/281 and pl. 38/281.

Max. h 2.5 cm., d of rim 16.4 cm., max. w 4.3 cm., max. th $0.7 \mathrm{~cm}$.

Light red (2.5YR 6/8) slip on exterior; red $(2.5 \mathrm{YR} 5 / 8)$ slip on interior. All of surface is burnished. Hard, thin paste, non-porous, fine, reddish yellow (5YR 6/8) fabric with some tiny lime inclusions.

282. (No. 1069): Rim fragment; Kepez, found in 2005. pl. 11/282 and pl. 38/282.

Max. h $3.8 \mathrm{~cm}$., d of rim $16.6 \mathrm{~cm}$., max. w 6.8 cm., max. th $0.5 \mathrm{~cm}$.

Red (2.5YR 5/6) slip on the exterior and interior rim. Their lower parts have yellow (10YR 7/6) unslipped surface on exterior and interior. The exterior surface is burnished, hard, sparsely porous, thin paste, fine, reddish yellow (5YR 6/6) fabric with some tiny lime inclusions.
283. (No. 1106): Rim fragment; Kepez, found in 2005. pl. 11/283 and pl. 38/283.

Max. h $2.2 \mathrm{~cm}$., d of rim $17.0 \mathrm{~cm}$., max. w 3.9 cm., max. th $0.6 \mathrm{~cm}$.

Reddish yellow (5YR 6/8) slip on exterior and interior rim. Reddish yellow (5YR 7/6) unslipped surface on interior. Exterior surface and interior rim are burnished. Average hardness; thin paste, very sparsely porous, fine, reddish yellow (5YR 6/8) fabric with infrequent sand inclusions.

284. (No. 1369): Rim fragment; Kepez, Necropolis, found in 2005. pl. 11/284 and pl. 38/284.

Max. h $2.8 \mathrm{~cm}$., d of rim $17.2 \mathrm{~cm}$., max. w 6.0 cm., max. th $0.8 \mathrm{~cm}$.

Light brown (7.5YR 6/4) unslipped surface on exterior; pale brown (10YR 6/3) unslipped surface on interior. Hard, thin paste, nonporous, fine, light brown (7.5YR 6/4) fabric with some medium grit and rare tiny lime inclusions.

Bowl Form 2 (pls. 11-12, nos. 286-300)

This is an outturned rim bowl shape with perhaps a hemispherical body and thin or medium walls. At Gordion four similar drinking vessels have been classified as hemispherical bowls where it has been interpreted as "a local variant" (Stewart 2010, 206, fig. 236, 371-374).

285. (No. 1061): Rim fragment; Kepez, found in 2005. pl. 38/285.

Max. h 1.7 cm., max. w 1.4 cm., max. th 0.4 $\mathrm{cm}$.

Red (10R 5/8) thin slip on exterior and interior. Soft, thin paste, non-porous, fine, red (10R $5 / 8$ ) fabric with rare quartz inclusions.

286. (No. 1195): Rim fragment; Kepez, Cistern, found in 2005. pl. 11/286 and pl. 38/286. 
Max. h 1.8 cm., max. w $1.6 \mathrm{~cm}$., max. th 0.5 $\mathrm{cm}$.

Red (2.5YR 5/8) slip on exterior and interior. Soft, thin paste, non-porous, fine, light red (2.5YR 6/8) fabric with rare sand inclusions.

287. (No. 581): Rim fragment; Kimistene, Acropolis, southern slope, just below the summit, up to Deresemail creek, found in 2005. pl. 38/287.

Max. h 1.4 cm., max. w 2.4 cm., max. th 0.6 $\mathrm{cm}$.

Red (2.5YR 5/8) shiny slip on exterior; light red (2.5YR 6/8) slip on interior. Hard, thin paste, non-porous, fine, red (2.5YR 5/8) fabric with frequent tiny lime and rare sand inclusions.

288. (No. 582): Rim fragment; Kimistene, Acropolis, southern slope, just below the summit, up to Deresemail creek, found in 2005. pl. 38/288.

Max. h $2.0 \mathrm{~cm}$., max. w $2.2 \mathrm{~cm}$., max. th 0.4 $\mathrm{cm}$.

Weak red (10R 5/4) slip on exterior and interior. Hard, thin paste, non-porous, fine, red (2.5YR 5/8) fabric with no visible inclusions.

289. (No. 685): Rim fragment; Kimistene, Acropolis, southern slope, just below the summit, up to Deresemail creek, found in 2005. pl. 38/289.

Max. h $2.0 \mathrm{~cm}$., d of base $7.8 \mathrm{~cm}$., max. w 2.3 cm., max. th $0.5 \mathrm{~cm}$.

Red (10R 4/6) slip on exterior and interior. Soft, thin paste, non-porous, fine, reddish brown (5YR 4/4) fabric with frequent micaceous and some lime inclusions.

290. (No. 1099): Rim fragment; Kepez, found in 2005. pl. 11/290 and pl. 38/290.

Max. h $2.3 \mathrm{~cm}$., max. w $2.0 \mathrm{~cm}$., max. th 0.8 $\mathrm{cm}$.
Red (10R 5/8) on exterior and interior rim. Its below part has reddish yellow (5YR 7/6) unslipped surface on interior. Average hardness; very sparsely porous, thin paste, fine, reddish yellow (5YR 6/6) fabric with rare lime inclusions.

291. (No. 1087): Rim fragment; Kepez, found in 2005. pl. 11/291 and pl. 38/291.

Max. h $2.5 \mathrm{~cm}$., max. w $2.1 \mathrm{~cm}$., max. th 0.4 $\mathrm{cm}$.

Red (2.5YR 5/6) slip on exterior and interior. Average hardness; thin paste, non-porous, fine, light brown (7.5YR 6/4) fabric with infrequent sand inclusions.

292. (No. 1020): Rim fragment; Kepez, found in 2005. pl. 11/292 and pl. 38/292.

Max. h $3.2 \mathrm{~cm}$., max. w $2.8 \mathrm{~cm}$., max. th 0.6 $\mathrm{cm}$.

Red (10R 5/6) slip on exterior and interior. Hard, thin paste, non-porous, fired to light reddish brown (10YR 6/3) and reddish yellow (5YR 6/6) fabric with some tiny lime and occasional sand inclusions.

293. (No. 1188): Rim fragment; Kepez, Cistern, found in 2005. pl. 11/293 and pl. 38/293. Max. h $3.8 \mathrm{~cm}$., max. w $2.8 \mathrm{~cm}$., max. th 0.5 $\mathrm{cm}$.

Red (2.5YR 5/8) slip on exterior and interior. Average hardness; thin paste, non-porous, fine, red $(2.5 \mathrm{YR} 5 / 6)$ fabric with some sand inclusions.

294. (No. 1128): Rim fragment; Kimistene, Acropolis, southern slope, just below the summit, up to Deresemail creek, found in 2005. pl. 11/294 and pl. 38/294.

Max. h 3.1 cm., max. w $3.2 \mathrm{~cm}$., max. th 0.4 $\mathrm{cm}$.

Red (10R 5/8) on exterior rim and interior rim. Their below parts have reddish yellow 
(7.5YR 7/6) unslipped surface, Average hardness; non-porous, thin paste, fine, reddish yellow (5YR 6/6) fabric with no visible inclusions.

295. (No. 1015): Rim fragment; Kepez, found in 2005. pl. 11/295 and pl. 39/295.

Max. h $5.5 \mathrm{~cm}$., d of rim $13.0 \mathrm{~cm}$., max. w 4.9 cm., max. th $0.5 \mathrm{~cm}$.

Brown (7.5YR 4/3) and reddish yellow (5YR 6/6) mottled slip on exterior; reddish yellow (5YR 6/6) band on interior rim. Its below part has pink (7.5YR 7/4) slip on interior. Hard, thin paste, non-porous, fine, pink (7.5YR 7/4) fabric with infrequent sand and rare lime inclusions.

296. (No. 1111): Rim fragment; Kepez, Cistern, found in 2005. pl. 12/296 and pl. 39/296. Max. h 1.7 cm., d of rim 13.4 cm., max. w $2.9 \mathrm{~cm}$., max. th $0.5 \mathrm{~cm}$.

Red (2.5YR 5/6) slip on exterior and interior. Average hardness; thin paste, non-porous, fine, reddish yellow (5YR 6/6) fabric with rare tiny lime inclusions.

297. (No. 1206): Rim fragment; Kimistene, summit of the Acropolis, southern slope, found in 2005. pl. 12/297 and pl. 39/297.

Max. h $2.3 \mathrm{~cm}$., d of rim $14.0 \mathrm{~cm}$., max. w 5.4 cm., max. th $0.5 \mathrm{~cm}$.

Red (2.5YR 5/6) slip on exterior; reddish yellow (5YR 6/6) slip on interior. Average hardness; thin paste, non-porous, fine, light brown (7.5YR 6/4) fabric with frequent tiny lime inclusions.

298. (No. 796): Rim fragment; Kimistene, Acropolis, southern slope, just below the summit, up to Deresemail creek, found in 2005. pl. 12/298 and pl. 39/298.

Max. h 3.8 cm., d of rim 14.8 cm., max. w 3.6 cm., max. th $0.5 \mathrm{~cm}$.
Red (2.5YR 5/6) on exterior rim and interior rim. Their below parts have reddish yellow (7.5YR 7/6) unslipped surface, hard, very sparsely porous, thin paste, fine, reddish yellow (5YR 6/6) fabric with frequent lime inclusions.

299. (No. 1159): Rim fragment; Kepez, Cistern, found in 2005. pl. 12/299 and pl. 39/299. Max. h $7.3 \mathrm{~cm}$., d of rim $15.2 \mathrm{~cm}$., max. w 5.4 cm., max. th $0.6 \mathrm{~cm}$.

Reddish yellow (7.5YR 7/6) and red (2.5YR $5 / 8)$ mottled slip on the exterior. Red (2.5YR 5/6) slip on the interior. Hard, very sparsely porous, thin paste, fine, reddish yellow (7.5YR 6/6) fabric with infrequent small grit and lime inclusion.

300. (No. 1112): Rim fragment; Kepez, Cistern, found in 2005. pl. 12/300 and pl. 39/300. Max. h 2.4 cm., d of rim $16.0 \mathrm{~cm}$., max. w 3.5 cm., max. th $0.6 \mathrm{~cm}$.

Reddish yellow (5YR 6/6) abrasion thin slip on exterior and interior. Very pale brown (10YR 8/2) band on interior slip. Hard, thin paste, non-porous, fine light brown (7.5YR 6/4) fabric with rare tiny lime inclusions.

Dish Form 1 (pl. 12, nos. 301-303)

Rounded rim of a deep dish form. The interior of no. 302 is painted.

301. (No. 1000): Rim fragment; Kepez, found in 2005. pl. 12/301 and pl. 39/301.

Max. h $5.4 \mathrm{~cm}$., max. w $4.8 \mathrm{~cm}$., max. th 0.4 $\mathrm{cm}$.

Very pale brown (10YR 8/2) slip on exterior; reddish yellow (7.5YR 7/6) slip on interior. Three parallel horizontal bands are very dark grey $(7.5 \mathrm{YR} 3 / 1)$. There is a red $(2.5 \mathrm{YR} 5 / 6)$ horizontal band between two bands on exterior. Hard, thin paste, very sparsely porous, 
fired to reddish yellow $(7.5 \mathrm{YR} 7 / 6)$ and reddish yellow (5YR 6/8) fabric with rare micaceous and sand inclusions.

302. (No. 936): Rim fragment; Kimistene, Acropolis, found in 2005. pl. 12/302 and pl. 39/302.

Max. h $1.7 \mathrm{~cm}$., d of rim $19.2 \mathrm{~cm}$. , max. w 3.5 cm., max. th $0.7 \mathrm{~cm}$.

Very pale brown (10YR 7/4) unslipped surface on exterior; reddish yellow (5YR 6/6) abrasion slip on interior. Hard, thin paste, very sparsely porous, fine, reddish yellow (5YR 6/6) fabric with some micaceous and tiny lime inclusions.

303. (No. 1092): Rim fragment; Kepez, found in 2005. pl. 12/303 and pl. 39/303.

Max. h 2.2 cm., d of rim 21.0 cm., max. w 7.9 cm., max. th $0.5 \mathrm{~cm}$.

Entire surface slightly slipped. Red (2.5YR 5/6) abrasion slip on the exterior; red (2.5YR 5/6) slip on the interior. Hard, very sparsely porous, thin paste; fine, reddish yellow (5YR $6 / 8$ ) fabric with rare tiny lime inclusion.

Dish Form 2 (pl. 13, nos. 304-305)

Rounded rim dishes with a straight edge and shallow surface. Their exterior is slipped with light colours.

304. (No. 1100): Rim fragment; Kepez, found in 2005. pl. 13/304 and pl. 39/304.

Max. h 2.3 cm., max. w $3.1 \mathrm{~cm}$., max. th 0.5 $\mathrm{cm}$.

Pink (7.5YR 7/4) slip on exterior and interior; red (2.5YR 5/6) on interior slip. Average hardness; non-porous, thin paste, fine, pink (7.5YR 7/4) fabric with infrequent lime inclusions.
305. (No. 1107): Rim fragment; Kepez, Cistern, found in 2005. pl. 13/305 and pl. 39/305. Max. h $3.0 \mathrm{~cm}$., max. w $3.3 \mathrm{~cm}$., max. th 0.6 $\mathrm{cm}$.

Light brown (7.5YR 6/4) slip on exterior and lower interior; red (2.5YR 5/6) on interior rim. Exterior surface is burnished. Hard, nonporous, thin paste, fine, pink (7.5YR 7/4) fabric with rare lime inclusions.

Plate (pl. 13, nos. 306-309)

Plates with an outcurved rim and shallow body. Similar to the shape of a fish plate, which was a fairly popular shape in the eastern Mediterranean during the Hellenistic period (Stewart 2010, 179).

306. (No. 1476): Rim fragment; Kimistene, Acropolis, southern slope, just below the summit, up to Deresemail creek, found in 2005. pl. 13/306 and pl. 39/306.

Max. h $0.9 \mathrm{~cm}$., max. w $3.5 \mathrm{~cm}$., max. th 0.5 $\mathrm{cm}$.

Pink (5YR 7/4) slip all of surface; reddish brown (2.5YR 5/4) on interior rim. Hard, thin paste, very sparsely porous, fired to red (2.5YR 5/6) and light brown (7.5YR 6/4) fabric with rare lime inclusions.

307. (No. 1336): Rim fragment; Kimistene, Cistern, eastern slope, found in 2005. pl. 13/307 and pl. 39/307.

Max. h 1.7 cm., d of rim $18.0 \mathrm{~cm}$., max. w 4.4 cm., max. th $0.5 \mathrm{~cm}$.

Red (2.5YR 6/8) slip on exterior; red (2.5YR $5 / 8$ ) shiny slip on interior. Hard, thin paste, non-porous, fine, red (2.5YR 6/8) fabric with no visible inclusions.

308. (No. 1088): Rim fragment; Kepez, found in 2005. pl. 13/308 and pl. 39/308.

Max. h 1.3 cm., d of rim 18.0 cm., max. w 7.0 
cm., max. th $0.5 \mathrm{~cm}$.

Very pale brown (10YR 7/3) thin slip on exterior; red (2.5YR 5/6) slip on interior. Pinkish white $(7.5 Y R$ 8/2) band on interior. Average hardness; very sparsely porous, thin paste, fine, light reddish brown (5YR 6/4) fabric with some sand and lime. A little micaceous inclusion.

Parallel: Stewart 2010, 203, fig. 232, 356 (P 4186; Middle Hellenistic).

309. (No. 1081): Rim fragment; Kepez, found in 2005. pl. 13/309 and pl. 39/309.

Max. h 0.9 cm., d of rim $23.0 \mathrm{~cm}$., max. w 3.4 cm., max. th $0.6 \mathrm{~cm}$.

Red (2.5YR 5/8) slip on exterior and interior. Average hardness; thin paste, non-porous, fine, reddish yellow (7.5YR 6/6) fabric with rare lime inclusions.

Base Fragments of Open Forms (pls. 1314 , nos. 310-314)

Their forms cannot be assigned; most of them should, however, be bowls. The most important characteristic of this form is a band decoration. Their measurements are similar to each other.

310. (No. 1009): Base fragment; Kepez, found in 2005. pl. 13/310 and pl. 39/310.

Max. h $2.1 \mathrm{~cm}$., d of base $7.2 \mathrm{~cm}$., max. w 4.3 cm., max. th $1.1 \mathrm{~cm}$.

Pink (5YR 7/4) slip on exterior; surface thinly slipped; red (2.5YR 5/6) slip on interior. Average hardness; non-porous, thin paste, fine, reddish yellow (5YR 6/6) fabric with some small sand, frequent lime inclusions.

311. (No. 1157): Base fragment; Kepez, Cistern, found in 2005. pl. 13/311 and pl. 39/311. Max. h $2.8 \mathrm{~cm}$., d of base $6.0 \mathrm{~cm}$., max. w 7.0 cm., max. th $0.9 \mathrm{~cm}$.

Reddish yellow (7.5YR 7/6) slip on the exte- rior; very pale brown (10YR 7/4) unslipped surface on the interior. There is a reddish yellow (7.5YR 7/6) concentric band on the interior surface. Hard, sparsely porous, fine, light red (2.5YR 6/6) fabric with frequent lime inclusions.

312. (No. 1016): Base fragment; Kepez, found in 2005. pl. 13/312 and pl. 39/312.

Max. h $2.2 \mathrm{~cm}$., d of base $10.8 \mathrm{~cm}$., max. w $6.4 \mathrm{~cm}$., max. th $0.9 \mathrm{~cm}$.

Pink (7.5YR 8/4) unslipped surface on exterior; red (10R 5/6) slip on interior. Hard, thin paste, non-porous, fine, reddish yellow (5YR 7/8) fabric with rare sand inclusions.

313. (No. 1198): Base fragment; Kepez, Cistern, found in 2005. pl. 14/313 and pl. 39/313. Max. h $2.8 \mathrm{~cm}$., max. w $9.0 \mathrm{~cm}$., max. th 1.2 $\mathrm{cm}$.

Very pale brown (10YR 7/3) slip on the exterior; very pale brown (10YR 7/3) slip on the centre of the bottom. Very pale brown (10YR $8 / 2)$ on the interior surface. A red (2.5YR 5/8) and dusky red (2.5YR 3/2) mottled-painted concentric band on the interior surface. Stripe caused by careless execution. Hard, very sparsely porous, thin paste, fine, pink (5YR 7/4) fabric with frequent micaceous and grit inclusions.

314. (No. 829): Base fragment; Kimistene, Cistern, eastern slope, found in 2005. pl. 14/314 and pl. 40/314.

Max. h $6.0 \mathrm{~cm}$., d of base $9.8 \mathrm{~cm}$., max. w 9.5 cm., max. th $0.6 \mathrm{~cm}$.

Reddish yellow (5YR 6/6) slip on the exterior; surface thinly slipped, light red $(2.5 \mathrm{YR}$ 6/8) slip on the interior. Weak red (7.5R 4/4) on the centre of the bottom; two concentric bands in dark reddish grey $(2.5 \mathrm{YR} 3 / 1)$ and very dark grey $(7.5 \mathrm{YR} 3 / 1)$ paint on the interior. Hard, non-porous, thin paste, fine, light 
red (2.5YR 6/8) fabric with occasional tiny lime and a little micaceous inclusion.

\section{Body Fragments of Open Forms (pls. 14-} 15 , nos. 328-373)

315. (No. 1064): Body fragment; Kepez, found in 2005. pl. 40/315.

Max. h 1.4 cm., max. w 1.6 cm., max. th 0.4 $\mathrm{cm}$.

Red (10R 5/8) slip on exterior and interior. Hard, thin paste, non-porous, fine, light red (2.5YR 6/8) fabric with frequent tiny sand and rare micaceous inclusions.

317. (No. 1063): Body fragment; Kepez, found in 2005. pl. 40/316.

Max. h $1.5 \mathrm{~cm}$., max. w $2.0 \mathrm{~cm}$., max. th 0.5 $\mathrm{cm}$.

Red (10R 5/8) slip on exterior and interior. Average hardness; thin paste, non-porous, fine, light red (2.5YR 6/8) fabric with no visible inclusions.

316. (No. 1189): Body fragment; Kepez, Cistern, found in 2005. pl. 40/317.

Max. h 2.2 cm., max. w 1.4 cm., max. th 0.4 $\mathrm{cm}$.

Very pale brown (10YR 8.5/2) slip on exterior; reddish yellow (7.5YR 7/6) unslipped surface on interior. A band in reddish brown (2.5YR 4/3) on exterior. Average hardness; thin paste, very sparsely porous, fine, reddish yellow (7.5YR 7/6) fabric with some micaceous inclusions.

318. (No. 1243): Body fragment; Kepez, found in 2005. pl. 40/318.

Max. h 1.4 cm., max. w $2.2 \mathrm{~cm}$., max. th 0.3 $\mathrm{cm}$.

Very pale brown (10YR 8/2) slip on lower exterior. A band in reddish brown (2.5YR 5/4) paint on upper exterior and interior. Hard, thin paste, non-porous, fine, pink (5YR 7/4) fabric with no visible inclusions.

319. (No. 1192): Body fragment; Kepez, Cistern, found in 2005. pl. 40/319.

Max. h 2.1 m., max. w 1.9 cm., max. th 0.6 $\mathrm{cm}$.

Red (10R 5/8) slip on exterior and interior. Average hardness; thin paste, non-porous, fired to reddish yellow (5YR 7/8-7.5YR 6/6) fabric with some lime and sand inclusions.

320. (No. 1050): Body fragment; Kepez, found in 2005. pl. 40/320.

Max. h $1.8 \mathrm{~cm}$., max. w $2.2 \mathrm{~cm}$., max. th 0.3 $\mathrm{cm}$.

Reddish brown (5YR 5/4) and reddish yellow (5YR 6/6) mottled slip on exterior. Reddish yellow (5YR 6/6) slip on interior. Two bands in pinkish white (7.5YR 8/2) paint on exterior slip. Hard, thin paste, non-porous, fine, pink (7.5YR 7/4) fabric with frequent tiny lime inclusions.

321. (No. 1241): Body fragment; Kepez, found in 2005. pl. 40/321.

Max. h $2.1 \mathrm{~cm}$., max. w 1.9 cm., max. th 0.5 $\mathrm{cm}$.

Red (2.5YR 5/8) shiny slip on exterior and interior. Hard, thin paste, sparsely porous, fine, light red (2.5YR 6/6) fabric with rare micaceous inclusions

322. (No. 1035): Body fragment; Kepez, found in 2005. pl. 40/322.

Max. h $2.1 \mathrm{~cm}$., max. w $2.3 \mathrm{~cm}$., max. th 0.8 $\mathrm{cm}$.

Red (10R 5/8) slip on exterior; light red (2.5YR 6/8) slip on interior. Average hardness; very sparsely porous, thin paste, nonporous, fine, reddish yellow (5YR 6/6) fabric with no visible inclusions. 
323. (No. 1025): Body fragment; Kepez, found in 2005. pl. 40/323.

Max. h $2.4 \mathrm{~cm}$., max. w $2.1 \mathrm{~cm}$., max. th 0.5 $\mathrm{cm}$.

Red (2.5YR 5/8) slip on exterior and interior. Average hardness; thin paste, non-porous, fine, reddish yellow (5YR 6/6) fabric with infrequent sand inclusions.

324. (No. 1022): Body fragment; Kepez, found in 2005. pl. 40/324.

Max. h $2.0 \mathrm{~cm}$., max. w $3.1 \mathrm{~cm}$., max. th 0.6 $\mathrm{cm}$.

Red (2.5YR 5/6) slip on exterior and interior. Hard, thin paste, non-porous, fine, very pale brown (10YR 7/3) fabric with no visible inclusions.

325. (No. 1148): Body fragment; Kimistene, Acropolis, temple slope, first slope on the north, found in 2005. pl. 40/325.

Max. h $2.1 \mathrm{~cm}$., max. w $2.6 \mathrm{~cm}$., max. th 0.6 $\mathrm{cm}$.

Red (2.5YR 5/6) slip on exterior and interior. Hard, thin paste, non-porous, fine, very pale brown (10YR 7/4) fabric with no visible inclusions.

326. (No. 1021): Body fragment; Kepez, found in 2005. pl. 40/326.

Max. h $2.4 \mathrm{~cm}$., max. w $2.5 \mathrm{~cm}$., max. th 0.6 $\mathrm{cm}$.

Red (2.5YR 5/8) slip on exterior and interior. Hard, thin paste, non-porous, fired to reddish yellow (5YR 6/6) and light red (2.5YR 6/8) mottled fabric with occasional sand inclusions.

327. (No. 1184): Body fragment; Kepez, surface find in the Cistern, found in 2005. pl. 40/327.

Max. h $3.0 \mathrm{~cm}$., max. w $2.0 \mathrm{~cm}$., max. th 0.5 $\mathrm{cm}$.
Weak red (10R 5/4) abrasion slip on exterior. Pink (7.5YR 7/4) slip on interior. Interior surface is burnished. Hard, thin paste, nonporous, fine, red (2.5YR 5/8) fabric with no visible inclusions.

328. (No. 1053): Body fragment; Kepez, found in 2005. pl. 14/328 and pl. 40/328.

Max. h 2.7 cm., max. w $2.4 \mathrm{~cm}$., max. th 0.5 $\mathrm{cm}$.

Reddish yellow (7.5YR 7/6) slip on exterior and interior. Three bands in red (2.5YR 5/6) on upper exterior and upper interior. Hard, thin paste, non-porous, fine, reddish yellow (7.5YR 6/6) fabric with rare sand inclusions.

329. (No. 1029): Body fragment; Kepez, found in 2005. pl. 40/329.

Max. h 2.4 cm., max. w 2.7 cm., max. th 0.4 $\mathrm{cm}$.

Shiny red (2.5YR 5/6) and reddish brown (2.5YR 4/4) mottled slip on exterior; red (2.5YR 4/8) slip on interior; its below part has reddish brown (5YR 4/3) slip on interior. Hard, thin paste, non-porous, fired to red (2.5YR 5/6) and light red (2.5YR 6/6) mottled fabric with rare lime inclusions.

330. (No. 1040): Body fragment; Kepez, found in 2005. pl. 14/330 and pl. 40/330.

Max. h $2.5 \mathrm{~cm}$., max. w $2.9 \mathrm{~cm}$., max. th 0.5 $\mathrm{cm}$.

Light red (2.5YR 6/8) on upper exterior; its below part has reddish yellow (5YR 7/6) slipped. Exterior surface is burnished. Light red (2.5YR 6/6) on upper interior; pinkish white $(7.5 Y R$ 8/2) band on interior slip. Its below part has very pale brown (10YR 7/4) unslipped surface on interior. Hard, very sparsely porous, thin paste, fine, reddish yellow (5YR 6/8) fabric with frequent tiny lime inclusions. 
331. (No. 1056): Body fragment; Kepez, found in 2005. pl. 40/331.

Max. h $2.8 \mathrm{~cm}$., max. w $2.8 \mathrm{~cm}$., max. th 0.9 $\mathrm{cm}$.

Very pale brown (10YR 7/4) unslipped surface on exterior; red (2.5YR 5/6) slip on interior. Hard, thin paste, non-porous, fine, very pale brown (10YR 7/4) fabric with occasional lime inclusions.

332. (No. 1032): Body fragment; Kepez, found in 2005. pl. 40/332.

Max. h 3.2 cm., max. w 2.4 cm., max. th 0.5 $\mathrm{cm}$.

Very pale brown (10YR 8/4) slip on exterior; red (2.5YR 5/8) slip on interior. A band in red (2.5YR 5/6) on exterior. Hard, thin paste, very sparsely porous, fine, reddish yellow (5YR 6/8) fabric with no visible inclusions.

333. (No. 1238): Body fragment; Kepez, found in 2005. pl. 40/333.

Max. h $3.4 \mathrm{~cm}$., max. w $2.7 \mathrm{~cm}$., max. th 0.6 $\mathrm{cm}$.

Red (2.5YR 5/6) and yellow (10YR 7/6) mottled slip on exterior; reddish yellow (5YR 6/6) slip on interior. Hard, thin paste, non-porous, fine, reddish yellow (7.5YR 6/6) fabric with rare micaceous, quartz and sand inclusions.

334. (No. 1176): Body fragment; Kepez, Cistern, found in 2005. pl. 40/334.

Max. h $2.8 \mathrm{~cm}$., max. w $2.8 \mathrm{~cm}$., max. th 0.7 $\mathrm{cm}$.

Red (2.5YR 5/6) slip on exterior; pink (5YR $7 / 4$ ) unslipped surface on interior. A band in red (2.5YR 5/8) on interior. Hard, thin paste, non-porous, fine, light red (2.5YR 6/6) fabric with some sand and lime inclusions.

335. (No. 518): Body fragment; Kimistene, Acropolis, southern slope, just below the summit, up to Deresemail creek, found in 2005. pl. 40/335.

Max. h 3.2 cm., max. w 2.2 cm., max. th 0.8 $\mathrm{cm}$.

Reddish brown (2.5YR 5/4) slip on exterior; light red (2.5YR 6/6) slip on interior. Hard, thin paste, very sparsely porous, fine, reddish yellow (5YR 6/6) fabric with some tiny lime inclusions.

336. (No. 1045): Body fragment; Kepez, found in 2005. pl. 40/336.

Max. h $3.2 \mathrm{~cm}$., max. w $2.3 \mathrm{~cm}$., max. th 0.5 $\mathrm{cm}$.

Very pale brown (10YR 7/4) thin slip on exterior; red (2.5YR 5/6) slip on interior. Hard, thin paste, non-porous, fired to reddish yellow (5YR 6/8) fabric with rare tiny lime inclusions.

337. (No. 1034): Body fragment; Kepez, found in 2005. pl. 40/337.

Max. h $2.5 \mathrm{~cm}$., max. w $3.4 \mathrm{~cm}$., max. th 0.5 $\mathrm{cm}$.

Reddish brown (5YR 5/4) unslipped surface on exterior, reddish yellow (7.5YR 6/6) slip on interior. Interior surface is burnished. Hard, thin paste, non-porous, fired to yellowish red (5YR 5/6) fabric with rare small grit and lime inclusions.

338. (No. 611): Body fragment; Kimistene, Acropolis, southern slope, just below the summit, up to Deresemail creek, found in 2005. pl. 40/338.

Max. h 2.4 cm., max. w $3.6 \mathrm{~cm}$., max. th 0.6 $\mathrm{cm}$.

Pink (7.5YR 7/4) thin slip on exterior; red (2.5YR 5/6) slip on interior. Hard, thin paste, non-porous, fine, yellowish red (5YR 5/6) fabric with some lime inclusions.

339. (No. 813): Body fragment; Kimistene, summit of the Acropolis, western slope, found in 2005. pl. 40/339. 
Max. h $2.5 \mathrm{~cm}$., max. w $3.6 \mathrm{~cm}$., max. th 0.5 $\mathrm{cm}$.

Reddish brown (5YR 5/4) abrasion slip on exterior; light red (2.5YR 6/8) slip on interior. Hard, thin paste, very sparsely porous, fine, pink (7.5YR 7/4) fabric with no visible inclusions.

340. (No. 1127): Body fragment; Kimistene, Acropolis, found in 2005. pl. 40/340.

Max. h $2.4 \mathrm{~cm}$., max. w $3.6 \mathrm{~cm}$., max. th 0.6 $\mathrm{cm}$.

Pink (7.5YR 7/4) unslipped surface on exterior; red (2.5YR 5/6) slip on interior. Hard, thin paste, non-porous, fine, reddish brown $(5 \mathrm{YR}$ $5 / 4$ ) fabric with occasional lime and rare sand inclusions.

341. (No. 1475): Body fragment; Kepez, in a grave assemblage, found in 2005. pl. 40/341. Max. h $2.5 \mathrm{~cm}$., max. w $3.2 \mathrm{~cm}$., max. th $0.7 \mathrm{~cm}$. Light red (2.5YR 6/6) unslipped surface on exterior and interior. A band in light red (2.5YR 6/8) on interior. Hard, thin paste, very sparsely porous, fired to yellowish red (5YR $5 / 8)$ and light brown (7.5YR 6/4) fabric with occasional sand inclusions.

342. (No. 511): Body fragment; Kimistene, Acropolis, southern slope, just below the summit, up to Deresemail creek, found in 2005. pl. 40/342.

Max. h $3.2 \mathrm{~cm}$., max. w $2.7 \mathrm{~cm}$., max. th 0.7 $\mathrm{cm}$.

Shiny red (10R 5/6) slip on exterior; matt weak red (10R 5/4) slip on interior. Hard, thin paste, non-porous, fine, reddish brown (5YR $5 / 4$ ) fabric with frequent tiny lime inclusions.

343. (No. 1234): Body fragment; Kepez, found in 2005. pl. 40/343.

Max. h $3.1 \mathrm{~cm}$., max. w $2.7 \mathrm{~cm}$., max. th 0.7 $\mathrm{cm}$.
Grey (7.5YR 5/4) unslipped surface on exterior; light brown (7.5YR 6/4) unslipped surface on interior. A band in reddish brown $(2.5 \mathrm{YR}$ $5 / 4$ ) on interior slip. Interior surface is burnished. Average hardness; sparsely porous, thin paste, fired to brown (7.5YR 5/3) fabric with some sand and occasional micaceous inclusions.

344. (No. 764): Body fragment; Kimistene, Acropolis, southern slope, just below the summit, up to Deresemail creek, found in 2005. pl. 40/344.

Max. h $3.3 \mathrm{~cm}$., max. w $2.9 \mathrm{~cm}$., max. th 0.6 $\mathrm{cm}$.

Reddish yellow (5YR 6/6) slip on exterior and interior. All of surface is thinly slipped. Exterior surface is burnished. Hard, thin paste, non-porous, fine, yellowish red (5YR 5/6) fabric with infrequent tiny lime and micaceous inclusions.

345. (No. 513): Body fragment; Kimistene, Acropolis, southern slope, just below the summit, up to Deresemail creek, found in 2005. pl. 40/345.

Max. h $3.0 \mathrm{~cm}$., max. w $3.2 \mathrm{~cm}$., max. th $0.5 \mathrm{~cm}$. Pink (5YR 7/4) unslipped surface on exterior, red (10R 5/6) slip on interior, hard, sparsely porous, thin paste, fine, light red (2.5YR 6/6) fabric with some sand inclusions.

346. (No. 1187): Body fragment; Kepez, surface find in the Cistern, found in 2005. pl. 40/346.

Max. h $3.2 \mathrm{~cm}$., max. w $3.0 \mathrm{~cm}$., max. th 0.6 $\mathrm{cm}$.

Red (2.5YR 5/6) slip on exterior, light red (2.5YR 6/6) slip on interior. Decoration: Two thin bands in reddish yellow (7.5YR 7/8) paint on slip, hard, very sparsely porous, thin paste, fine, reddish yellow (5YR 6/6) fabric with rare sand inclusions. 
347. (No. 1182): Body fragment; Kepez, surface find in the Cistern, found in 2005. It has been restored with no. 306. pl. 41/347.

Max. h 3.9 cm., max. w $2.7 \mathrm{~cm}$., max. th $0.6 \mathrm{~cm}$. Reddish yellow (5YR 6/6) slip on exterior, light grey (10YR 7/2) slip on interior. Interior surface is burnished. Decoration: Pinkish white (7.5YR 8/2) band on exterior slip. Hard, thin paste, very sparsely porous, fine, pink (7.5YR 7/4) fabric with some sand and rare small grit inclusions.

348. (No. 1018): Body fragment; Kepez, found in 2005. pl. 41/348.

Max. h 3.7 cm., max. w $3.2 \mathrm{~cm}$., max. th 0.5 $\mathrm{cm}$.

Very pale brown (10YR 8/2) slip on exterior, in red $(2.5$ YR 4/6) paint on exterior slip, red (10R 4/6) slip on interior, Average hardness; thin paste, very sparsely porous, fine, reddish yellow (7.5YR 7/6) fabric with some lime and sand inclusions.

349. (No. 490): Body fragment; Kimistene, Acropolis, southern slope, just below the summit, up to Deresemail creek, found in 2005. pl. 41/349.

Max. h 2.4 cm., max. w $3.6 \mathrm{~cm}$., max. th 0.6 $\mathrm{cm}$.

Light grey (10YR 7/2) abrasion slip on exterior; red (2.5YR 4/6) slip on interior. Exterior surface is burnished. Average hardness; thin paste, very sparsely porous, fine, light brown (7.5YR 6/3) fabric with no visible inclusions.

350. (No. 1031): Body fragment; Kepez, found in 2005. pl. 41/350.

Max. h 2.2 cm., max. w $4.8 \mathrm{~cm}$., max. th 0.5 $\mathrm{cm}$.

Pink (7.5YR 7/4) unslipped surface on exterior; weak red (10R 4/4) slip on exterior, hard. Thin paste, non-porous, fine, reddish yellow (5YR 6/8) fabric with no visible inclusions.
351. (No. 1467): Rim fragment; Kimistene, Acropolis, southern slope, just below the summit, up to Deresemail creek, found in 2005. pl. 41/351.

Max. h 3.0 cm., max. w $4.1 \mathrm{~cm}$., max. th 0.6 $\mathrm{cm}$.

Pink (7.5YR 8/3) abrasion slip on exterior; red (2.5YR 5/6) slip on interior. Hard, sparsely porous, thin paste, fine, light red (2.5YR 6/6) fabric with rare micaceous inclusions.

352. (No. 504): Body fragment; Kimistene, Acropolis, southern slope, just below the summit, up to Deresemail creek, found in 2005. pl. 41/352.

Max. h 2.9 cm., max. w 3.9 cm., max. th 1.0 $\mathrm{cm}$.

Red (2.5YR 4/6) slip on exterior and interior. Hard, non-porous, fine, reddish yellow (5YR 6/6) fabric with rare tiny lime inclusions.

353. (No. 1014): Body fragment; Kepez, found in 2005. pl. 41/353.

Max. h 1.9 cm., max. w 3.9 cm., max. th 0.7 $\mathrm{cm}$.

Red (2.5YR 5/6) and reddish yellow (7.5YR 7/6) mottled slip on exterior; red (2.5YR 5/6) slip on interior. Hard, thin paste, non-porous, fine, light reddish brown (5YR 6/4) fabric with frequent tiny lime inclusions.

354. (No. 1179): Body fragment; Kepez, Cistern, found in 2005. pl. 14/354 and pl. 41/354. Max. h 1.8 cm., max. w 4.3 cm., max. th 0.6 $\mathrm{cm}$.

Very pale brown (10YR 8/4) slip on exterior; very pale brown (10YR 7/4) slip on interior. A band in dusky red (2.5YR 3/2) on lower exterior. Exterior surface is burnished. Average hardness; thin paste, non-porous, fine, reddish yellow (7.5YR 6/6) fabric with some tiny lime and occasional sand inclusions. 
355. (No. 1005): Body fragment; Kepez, found in 2005. pl. 14/355 and pl. 41/355.

Max. h 1.4 cm., max. w $4.1 \mathrm{~cm}$., max. th 1.0 $\mathrm{cm}$.

Very pale brown (10YR 7/4) unslipped surface on exterior and interior. A band in reddish brown (5YR 5/4) on interior. Hard, thin paste, non-porous, fine, very pale brown (10YR 7/3) fabric with infrequent tiny grog and lime inclusions.

356. (No. 1185): Body fragment; Kepez, Cistern, found in 2005. pl. 41/356.

Max. h $3.0 \mathrm{~cm}$., max. w $3.6 \mathrm{~cm}$., max. th 0.6 $\mathrm{cm}$.

Pink (7.5YR 7/4) slip on exterior; red (2.5YR 5/6) slip on interior. Hard, very sparsely porous, thin paste, fine, reddish yellow (7.5YR 6/6) fabric with rare micaceous and some sand inclusions.

357. (No. 1324): Body fragment; Kimistene, summit of the Acropolis, southern slope, found in 2005. pl. 41/357.

Max. h $3.1 \mathrm{~cm}$, max. w $4.3 \mathrm{~cm}$., max. th 0.9 $\mathrm{cm}$.

Reddish yellow (5YR 6/6) unslipped surface on exterior; red (2.5YR 4/6) slip on interior. Hard, thin paste, non-porous, fine, reddish yellow (5YR 6/6) fabric with occasional sand and rare micaceous inclusions.

358. (No. 668): Body fragment; Kimistene, Acropolis, southern slope, just below the summit, up to Deresemail creek, found in 2005 . pl. 14/358 and pl. 41/358.

Max. h $2.8 \mathrm{~cm}$., max. w $3.9 \mathrm{~cm}$., max. th 1.0 $\mathrm{cm}$.

Very pale brown (10YR 7/3) slip on exterior; reddish yellow (7.5YR 6/6) slip on interior. A band in reddish brown (5YR 5/4) on interior. All of surface is burnished. Hard, thin paste, porous, fine, reddish yellow (5YR 6/6) fabric with some tiny lime and micaceous inclusions.

359. (No. 1131): Body fragment; Kimistene, Acropolis, southern slope, just below the summit, up to Deresemail creek, found in 2005. pl. 41/359.

Max. h $3.7 \mathrm{~cm}$., max. w $4.1 \mathrm{~cm}$., max. th 0.7 $\mathrm{cm}$.

Red (10R 5/6) slip on exterior and interior. Hard, thin paste, non-porous, fine, light red (2.5YR 6/6) fabric with some tiny and rare small lime inclusions.

360. (No. 1175): Body fragment; Kepez, Cistern, found in 2005. pl. 41/360.

Max. h $3.8 \mathrm{~cm}$., max. w $3.9 \mathrm{~cm}$., max. th 0.8 $\mathrm{cm}$.

Yellowish red (5YR 5/6) slip on exterior and interior. All of surface is burnished. Average hardness; thin paste, non-porous, fired to yellowish red (5YR 5/6) fabric with frequent tiny lime inclusions.

361. (No. 1010): Body fragment; Kepez, found in 2005. pl. 15/361 and pl. 41/361.

Max. h $2.2 \mathrm{~cm}$., max. w $3.4 \mathrm{~cm}$., max. th 0.9 $\mathrm{cm}$.

Very pale brown (10YR 7/3) slip on exterior; light grey (10YR 7/2) slip on interior. A band in light reddish brown (5YR 6/4) on interior; this band is bordered with two thin bands in reddish brown (5YR 5/4). Average hardness; thin paste, non-porous, fine, yellowish red (5YR 5/6) fabric with no visible inclusions.

362. (No. 484): Body fragment; Kimistene, Acropolis, southern slope, just below the summit, up to Deresemail creek, found in 2005. pl. 41/362.

Max. h 4.5 cm., max. w $3.3 \mathrm{~cm}$., max. th 1.0 $\mathrm{cm}$.

Red (2.5YR 5/6) slip on exterior; reddish 
brown (2.5YR 4/4) slip on interior. Hard, nonporous, thin paste, fine, reddish yellow (5YR $6 / 6)$ fabric with some tiny lime inclusions.

363. (No. 1169): Body fragment; Kepez, Cistern, found in 2005. pl. 15/363 and pl. 41/363. Max. h $2.0 \mathrm{~cm}$., max. w $3.5 \mathrm{~cm}$., max. th 0.6 $\mathrm{cm}$.

Very pale brown (10YR 7/3) slip on exterior; pink (7.5YR 7/4) unslipped surface on interior. Interior surface separated with a band in light red (2.5YR 6/6). Hard, thin paste, nonporous, fine, reddish yellow (5YR 6/6) fabric with some tiny sand and lime inclusions.

364. (No. 1183): Body fragment; Kepez, Cistern, found in 2005. pl. 15/364 and pl. 41/364. Max. h $1.7 \mathrm{~cm}$., max. w $4.5 \mathrm{~cm}$., max. th 0.6 $\mathrm{cm}$.

Light red (2.5YR 7/6) slip on exterior and interior. Surface is thinly slipped. Two bands in red $(2.5 \mathrm{YR} 5 / 8)$ on interior. Hard, thin paste, sparsely porous, fine, light red (2.5YR 6/6) fabric with frequent sand and micaceous inclusions.

365. (No. 1368): Body fragment; Kepez, Necropolis, found in 2005. pl. 41/365.

Max. h 5.2 cm., max. w 4.4 cm., max. th 0.5 $\mathrm{cm}$.

Red (10R 5/6) slip on exterior and interior. Average hardness; thin paste, very sparsely porous, fine, reddish yellow (5YR 6/8) fabric with no visible inclusions.

366. (No. 1043): Body fragment; Kepez, found in 2005. pl. 15/366 and pl. 41/366.

Max. h $1.3 \mathrm{~cm}$., max. w $3.2 \mathrm{~cm}$., max. th 0.6 $\mathrm{cm}$.

Yellowish red (5YR 5/6) slip on exterior; light grey (10YR 7/2) slip on interior. Interior surface is burnished. Pinkish white (7.5YR 8/2) band on exterior slip. Hard, thin paste, very sparsely porous, fine, light brown (7.5YR 6/4) fabric with occasional sand inclusions.

367. (No. 561): Body and base fragment; Kepez, found in 2005. pl. 15/367 and pl. 41/367.

Max. h $2.4 \mathrm{~cm}$., max. w $5.1 \mathrm{~cm}$., max. th 1.0 $\mathrm{cm}$.

Very pale brown (10YR 7/4) slip on exterior; reddish yellow (7.5YR 7/6) unslipped surface on interior. A concentric band in red (2.5YR 5/6) on interior. Average hardness; very sparsely porous, thin paste, fired to reddish yellow (7.5YR 6/6) and yellowish red (5YR $5 / 6$ ) fabric with rare lime inclusions.

368. (No. 1007): Body fragment; Kepez, found in 2005. pl. 15/368 and pl. 41/368.

Max. h $3.0 \mathrm{~cm}$., max. w $6.1 \mathrm{~cm}$., max. th 0.9 $\mathrm{cm}$.

Very pale brown (10YR 7/3) thin slip on exterior and interior. A band in yellowish red (5YR 5/6) on interior. Average hardness; non-porous, thin paste, fine, very pale brown (10YR 7/3) fabric with infrequent micaceous inclusions.

369. (No. 1167): Body fragment; Kepez, Cistern, found in 2005. pl. 15/369 and pl. 41/369. Max. h $2.1 \mathrm{~cm}$., max. w $6.5 \mathrm{~cm}$., max. th 1.1 $\mathrm{cm}$.

Very pale brown (10YR 7/4) slip on exterior; reddish yellow (7.5YR 7/6) slip on interior. Two bands in light red (2.5YR 6/8) on interior. Hard, thin paste, sparsely porous, fine, reddish yellow (5YR 6/6) fabric with some tiny lime, occasional small grit and micaceous inclusions.

370. (No. 998): Body fragment; Kepez, found in 2005. pl. 15/370 and pl. 42/370.

Max. h $1.5 \mathrm{~cm}$., max. w $6.8 \mathrm{~cm}$., max. th 1.1 $\mathrm{cm}$. 
Very pale brown (10YR 7/4) unslipped surface on exterior and interior. Two bands in dark greyish brown (10YR 4/2) and light brown (7.5YR 6/4) paint on interior. Hard, thin paste, sparsely porous, fine, very pale brown (10YR 7/4) fabric with infrequent small lime and some medium grit inclusions.

371. (No.760): Body fragment; Kimistene, Acropolis, southern slope, just below the summit, up to Deresemail creek, found in 2005. pl. 42/371.

Max. h $5.5 \mathrm{~cm}$., max. w $6.6 \mathrm{~cm}$., max. th 0.9 $\mathrm{cm}$.

Very pale brown (10YR 7/4) unslipped surface on exterior. Smoothed surface on exterior; reddish brown (2.5YR 4/4) slip on interior. Hard, thin paste, non-porous, fired to light brown (7.5YR 6/4) and pale brown (10YR $6 / 3$ ) fabric with occasional lime, sand and rare quartz inclusions.

372. (No. 994): Body and base fragment; Kepez, found in 2005. pl. 15/372 and pl. 42/372.

Max. h $2.5 \mathrm{~cm}$., max. w $7.6 \mathrm{~cm}$., max. th 1.1 $\mathrm{cm}$.

Pink (5YR 7/4) unslipped surface on exterior; reddish yellow (5YR 6/6) unslipped surface on interior. A concentric band in red (2.5YR $5 / 6$ ) on interior. On the exterior some grooves as decoration. Average hardness; non-porous, thin paste, fired to reddish brown (5YR 5/4) fabric with frequent sand and some lime inclusions.

373. (No. 1393): Body fragment; Kimistene, summit of the Acropolis, surface find, found in 2005. pl. 15/373 and pl. 42/373.

Max. h 3.4 cm., max. w $6.2 \mathrm{~cm}$., max. th 1.0 $\mathrm{cm}$.

Reddish yellow (7.5YR 7/6) slip on exterior and interior. A concentric band in red (2.5YR
5/6) paint on interior. Hard, thin paste, sparsely porous, fine, reddish yellow (7.5YR 6/6) fabric with some tiny lime, sand inclusions.

CLOSED FORMS (pl. 16, nos. 374-395) / Rim Fragments of Closed Forms (pl. 16, nos. 374-376)

Pitcher or juglets with outcurved rims.

374. (No. 1101): Rim fragment; Kepez, found in 2005. pl. 16/374 and pl. 42/374.

Max. h $2.1 \mathrm{~cm}$., max. w $2.5 \mathrm{~cm}$., max. th 0.4 $\mathrm{cm}$.

Red (10R 5/6) slip on exterior; interior surface thinly slipped. Matt reddish yellow (5YR 7/6) slip on interior rim. Hard, non-porous, thin paste, fine, reddish yellow (5YR 7/6) fabric with no visible inclusions.

375. (No. 1002): Rim fragment of a closed form; Kepez, found in 2005. pl. 16/375 and pl. 42/375.

Max. h 4.4 cm., max. w $4.3 \mathrm{~cm}$., max. th 0.9 $\mathrm{cm}$.

Very pale brown (10YR 8/4) slip on the exterior. Pink (5YR 7/4) unslipped surface on the interior. Red (10R 5/6) on exterior rim; its lower part has a black (5YR 2.5/1) horizontal band on the exterior. Average hardness; thin paste, very sparsely porous, fired to reddish yellow (7.5YR 6/6) and light red (2.5YR 6/8) fabric with rare small grog and tiny lime; infrequent sand inclusions.

376. (No. 1083): Rim fragment of a closed form; Kepez, found in 2005. pl. 16/376 and pl. 42/376.

Max. h 2.4 cm., d of rim $7.8 \mathrm{~cm}$., max. w $3.1 \mathrm{~cm}$., max. th $0.6 \mathrm{~cm}$.

Light red (2.5YR 6/6) and red (2.5YR 5/6) mottled slip on exterior; red $(2.5 \mathrm{YR} 5 / 6)$ 
slip on interior rim. Its lower part has a pink (7.5YR 7/4) unslipped surface. On the neck a weak red (2.5YR 4/2) band. Hard, thin paste, non-porous, fine, reddish yellow (5YR 6/6) fabric with frequent tiny lime inclusion.

Handle Fragment of a Closed Form (pl. 16 , no. 377)

Vertical handle.

377. (No. 1239): Handle fragment; Kepez, found in 2005. pl. 16/377 and pl. 42/377.

Max. h 1.3 cm., max. w 2.2 cm., max. th 1.3 $\mathrm{cm}$.

Red (10R 4/6) slip on all of surface. Average hardness; non-porous, fine, red (2.5YR 5/6) fabric with frequent tiny lime inclusions.

\section{Body Fragments of Closed Forms (pl. 16,} nos. 385-395)

Body fragments of unidentified closed shapes. Their interiors are unslipped; exterior decorated mostly with bands.

378. (No. 1042): Body fragment; Kepez, found in 2005. pl. 42/378.

Max. h 3.0 cm., max. w $1.7 \mathrm{~cm}$., max. th 0.7 $\mathrm{cm}$.

Red (2.5YR 5/6) slip on exterior; pink (7.5YR 7/4) unslipped surface on interior. Soft, thin paste, non-porous, fine, red (2.5YR 5/8) fabric with frequent tiny lime inclusions.

379. (No. 1186): Body fragment; Kepez, Cistern, found in 2005. pl. 42/379.

Max. h $1.8 \mathrm{~cm}$., max. w $2.6 \mathrm{~cm}$., max. th $0.7 \mathrm{~cm}$. Red (2.5YR 5/8) slip on exterior; light grey (10YR 7/2) unslipped surface on exterior. Average hardness; thin paste, non-porous, fired to pale brown (10YR 6/3) mottled fabric with rare micaceous and sand inclusions.
380. (No. 791): Body fragment; Kimistene, Acropolis, southern slope, just below the summit, up to Deresemail creek, found in 2005. pl. 42/380.

Max. h 2.5 cm., max. w $2.6 \mathrm{~cm}$., max. th 0.5 $\mathrm{cm}$.

Very pale brown (10YR 8/3) slip on exterior; yellowish red (5YR 5/6) on exterior slip. Reddish yellow (7.5YR 7/6) unslipped surface on interior. Hard, thin paste, very sparsely porous, fine, pink (7.5YR 7/3) fabric with some sand and tiny lime inclusions.

381. (No. 1240): Body fragment; Kepez, found in 2005. pl. 42/381.

Max. h $2.4 \mathrm{~cm}$., max. w $3.1 \mathrm{~cm}$., max. th 0.5 $\mathrm{cm}$.

Red (10R 5/8) slip on upper exterior; its below part has reddish yellow (5YR 7/6) unslipped surface. Pink (7.5YR 7/4) unslipped surface on interior. Hard, thin paste, non-porous, fine, reddish yellow (5YR 6/6) fabric with occasional small lime inclusions.

382. (No. 1174): Body fragment; Kepez, Cistern, found in 2005. pl. 42/382.

Max. h $1.6 \mathrm{~cm}$., max. w $3.2 \mathrm{~cm}$., max. th 0.5 $\mathrm{cm}$.

Red (10R 5/8) slip on exterior; reddish yellow (5YR 7/6) unslipped surface on interior. A band in pinkish white (5YR 8/2) on exterior slip. Hard, non-porous, thin paste, reddish yellow (5YR 6/6) fabric with some small grit inclusions.

383. (No. 1411): Body fragment; Kepez, surface find, found in 2005. pl. 42/383.

Max. h 1.4 cm., max. w $4.0 \mathrm{~cm}$., max. th 0.6 $\mathrm{cm}$.

Red (10R 5/6) slip on exterior; pink (7.5YR $7 / 4$ ) unslipped surface on interior. Two bands in weak red (10R 4/2) and white (5YR 8/1) on exterior slip. Hard, very sparsely porous, thin 
paste, fired to reddish yellow (5YR 6/6) and light reddish brown (10YR 6/4) fabric with some sand, rare tiny lime inclusions.

384. (No. 1038): Body fragment; Kepez, found in 2005. pl. 42/384.

Max. h 3.5 cm., max. $2.7 \mathrm{~cm}$., max. th $0.6 \mathrm{~cm}$. Red (2.5YR 4/6) slip on exterior; light brown (7.5YR 6/4) unslipped surface on interior. A band in light brown (7.5YR 6/4) on slip. Average hardness; thin paste, non-porous, fine, brown (7.5YR 4/4) fabric with some lime and sand inclusions.

385. (No. 1409): Body fragment; Kepez, surface find, found in 2005. pl. 16/385 and pl. 42/385.

Max. h 3.4 cm., max. w 2.9 cm., max. th 0.6 $\mathrm{cm}$.

Pink (5YR 8/3) slip on exterior; pink (5YR 7/4) unslipped surface on interior. Three bands in black (7.5YR 2.5/1) and red (2.5YR $5 / 6$ ) on exterior. Shallow grooves on the surface. Hard, thin paste, non-porous, fine, yellowish red (5YR 5/6) fabric with some sand, tiny lime inclusions.

Parallel: Stewart 2010, 210-211, fig. 244, 392 (middle Hellenistic pitcher from Gordion).

386. (No. 523): Body fragment; Kimistene, Acropolis, southern slope, just below the summit, up to Deresemail creek, found in 2005.

pl. 16/386 and pl. 42/386.

Max. h $3.5 \mathrm{~cm}$., max. w $3.1 \mathrm{~cm}$., max. th 0.7 cm.

Red (10R 5/8) slip on upper exterior; light reddish brown (2.5YR 6/4) unslipped surface on interior. Two bands in pinkish white (7.5YR 8/2) paint on exterior slip. Hard, thin paste, non-porous, fine, reddish yellow (5YR 6/6) fabric with frequent tiny lime inclusions.
387. (No. 1327): Body fragment; Kimistene, Acropolis, southern slope, just below the summit, up to Deresemail creek, found in 2005. pl. 16/387 and pl. 42/387.

Max. h 3.5 cm., max. w $3.1 \mathrm{~cm}$., max. th 0.7 $\mathrm{cm}$.

Red (2.5YR 4/6) slip on exterior; reddish yellow (7.5YR 7/6) unslipped surface on interior. Three bands in dusky red (2.5YR 3/2) and pinkish white (7.5YR 8/2) on exterior. Hard, thin paste, non-porous, fine, light brown (7.5YR 6/4) fabric with some tiny lime and sand inclusions.

388. (No. 1468): Body fragment; Kimistene, Acropolis, southern slope, just below the summit, up to Deresemail creek, found in 2005. pl. 42/388.

Max. h $3.2 \mathrm{~cm}$., max. w $3.6 \mathrm{~cm}$., max. th 0.7 $\mathrm{cm}$.

Red (2.5YR 5/8) slip on exterior; reddish yellow (5YR 7/6) unslipped surface on interior. Hard, thin paste, very sparsely porous, fine, light red (2.5YR 6/8) fabric with frequent tiny lime and rare sand inclusions.

389. (No. 1012): Body fragment; Kepez, found in 2005. pl. 42/389.

Max. h 3.7 cm., max. w 3.6 cm., max. th 0.6 $\mathrm{cm}$.

Reddish brown (5YR 5/4) slip on exterior; pink (7.5YR 7/4) unslipped surface on interior. Hard, thin paste, non-porous, fine, reddish yellow $(7.5$ YR 6/6) fabric with frequent tiny lime inclusions.

390. (No. 890): Body fragment; Kimistene, Acropolis, southern slope, underground cave (cistern?), surface find, found in 2005. pl. 42/390.

Max. h 3.5 cm., max. w 4.0 cm., max. th 0.5 $\mathrm{cm}$.

Red (2.5YR 5/6) shiny slip on exterior; very 
pale brown (10YR 8/4) unslipped surface on interior. Hard, thin paste, non-porous, fine, light brown (7.5YR 6/4) fabric with occasional tiny lime inclusions.

391. (No. 1199): Body fragment; Kepez, Cistern, found in 2005. pl. 42/391.

Max. h 3.2 cm., max. w $4.0 \mathrm{~cm}$., max. th 1.2 $\mathrm{cm}$.

Red (2.5YR 5/6) slip on exterior; reddish yellow (7.5YR 7/6) unslipped surface on interior. Two bands in weak red (2.5YR 4/2) and pinkish white (7.5YR 8/2) on exterior slip. Average hardness; porous, thin paste, fine, reddish yellow (5YR 6/6) fabric with some micaceous inclusions.

392. (No. 1356): Body fragment; Kimistene, Cistern, eastern slope, found in 2005. pl. 42/392.

Max. h 4.5 cm., max. w $4.0 \mathrm{~cm}$., max. th 0.8 $\mathrm{cm}$.

Red (2.5YR 5/6) abrasion slip on exterior; reddish yellow (7.5YR 7/6) unslipped surface on interior. Hard, thin paste, sparsely porous, fine, reddish yellow (5YR 6/6) fabric with frequent tiny lime and calsite inclusions.

393. (No. 996): Body fragment; Kepez, found in 2005. pl. 16/393 and pl. 43/393.

Max. h $3.0 \mathrm{~cm}$., max. w $5.2 \mathrm{~cm}$., max. th 0.7 $\mathrm{cm}$.

Yellowish red (5YR 5/6) slip on exterior; very pale brown (10YR 7/4) unslipped surface on interior. Two bands in reddish brown (5YR 4/4) and pinkish white (7.5YR 8/2) on exterior slip. Average hardness; non-porous, thin paste, fine, light brown (7.5YR 6/4) fabric with some tiny lime and rare sand inclusions.

394. (No. 1008): Body fragment; Kepez, found in 2005. pl. 43/394.

Max. h $3.6 \mathrm{~cm}$., max. w $4.6 \mathrm{~cm}$., max. th $0.6 \mathrm{~cm}$.
Reddish brown (5YR 5/4) slip on exterior; light brown (7.5YR 6/4) unslipped surface on interior. A band in reddish yellow (7.5YR 6/6) on exterior slip. Hard, thin paste, very sparsely porous, fired to brown (7.5YR 5/4) and reddish brown (5YR 5/4) fabric with rare tiny lime inclusions.

395. (No. 1163): Body fragment; Kepez, Cistern, found in 2005. pl. 16/395 and pl. 43/395. Max. h $5.3 \mathrm{~cm}$., max. w $5.7 \mathrm{~cm}$., max. th 0.8 $\mathrm{cm}$.

Brown (7.5YR 4/3) shiny slip on upper exterior; brown (7.5YR 5/4) matt slip on lower exterior. Light brown (7.5YR 6/4) unslipped surface on interior. Three bands in pinkish white (7.5YR 8/2) and dusky red (2.5YR 3/2) on exterior. Hard, thin paste, very sparsely porous, fine, reddish yellow (7.5YR 6/6) fabric with frequent tiny lime inclusions.

X. LATE HELLENISTIC-EARLY ROMAN GREY WARE (pls. 16-18, no. 396-436)

In the Hellenistic period at Gordion nearly $75 \%$ of all vessels are grey (Stewart 2010, 146; and Voigt et al. 1997, 18-19) and grey vessels appear in every general functional category (Stewart 2010, 146). At Hadrianopolis the earliest Roman fineware is represented by the grey ware (as well as brown-slipped ware), dating between the 1st century B.C. to the 2 nd century A.D. (i.e., for proto-sigillata phase) which was almost exclusively locally produced, following the Late Iron Age (so-called "Phrygian") tradition in production technics, i.e. manufacture as well as in the development of types. S. Mitchell believed there was no Hellenistic phase at Hadrianopolis (Mitchell 1993, 93); but we have a few Late Hellenistic pottery sherds (i.e from the 1 st cent. B.C.) from Hadrianopolis. Through these finds it is possible to contruct the first settlement at Hadrianopolis 
in the 1st century B.C. Since Kimistene and other nearby settlements in Hadrianopolis's chora also yielded pottery of the Bronze and Iron Ages, it is surprizing to observe the physical similarity in appearance between Late Hellenistic-Early Roman grey ware in Hadrianopolis and earlier grey wares in its chora. During the Byzantine period there is a certain grey ware tradition in the region as well. The most important difference between this later grey ware and Late Hellenistic ones is speed of the wheel and its traces on the surface.

The paste of this ware is grey (10YR 5/16/1, 2.5Y 5/1-6/1, Gley $15 / \mathrm{N}$ ), dark grey (10YR $4 / 1,2.5 \mathrm{Y} 4 / 1$, Gley $14 / \mathrm{N}$ ), very dark grey (Gley $13 / \mathrm{N})$, greyish brown (10YR 5/2), black (Gley 1 $2.5 / \mathrm{N}$ ), bluish grey (Gley $25 / 5 \mathrm{~PB}$ ), pale brown (10YR 6/3) and light brown (7.5YR 6/4). Fine and hard paste. Bad fired samples are mottled in grey, brown or dark greyish brown. Some samples have no inclusions; some of them have mica, lime and sand in medium sizes on their surface. Surfaces are porous. Its fabric is similar to local sigillata of our study region. Slip is close to metallic tones, such as very dark gary (10YR 3/1, 2.5Y 3/1, Gley $13 / \mathrm{N})$, dark grey (10YR 4/1, 2.5Y 4/1, Gley 1 4/N), grey (10YR 5/1, 2.5Y 5/1-6/1, Gley $16 / \mathrm{N})$, black (2.5Y 2.5/1, Gley $12.5 / \mathrm{N})$, dark reddish grey (5YR 4/2), greyish brown (10YR 5/2) and dark greyish brown (2.5Y 4/2). Slip had been applied in dipping position and in careless manner. Wall thickness differs between 0.3-1.4 cms. Most of the open vessels belong to a certain plate form.

55 sherds in total; 26 of which are open and 29 of them are closed forms. Most of them were found at Hadrianopolis (22 from domus, 10 from the Bath A, 8 from the Bath B, 8 from western tomb and 2 from the absidal building) as well as Kimistene, Kepez and Boncuklar.

They are dated to the 1st century B.C. to 2nd century A.D.
OPEN FORMS (pls. 16-22, nos. 396-418)

/ Bowl Form 1 (pl. 16, nos. 396-398)

396. (No. 110): Rim fragment; Bath $B$, Room 6, found in 2007.pl. 16/396 and pl. 43/396. Max. h 3.7 cm., max. w 4.2 cm., max. th 0.3 $\mathrm{cm}$.

Dark grey (10YR 4/1) slip on exterior; very dark grey (Gley $13 / \mathrm{N}$ ) slip on interior. Hard, thin paste, non-porous, fine, dark grey $(2.5 \mathrm{Y}$ $4 / 1$ ) fabric with rare micaceous inclusion.

397. (No. 100): Rim fragment; Bath B, Room 6, found in 2007. pl. 16/397 and pl. 43/397.

Max. h $3.1 \mathrm{~cm}$., max. w $4.6 \mathrm{~cm}$., max. th 0.5 $\mathrm{cm}$.

Very dark grey (Gley $13 / \mathrm{N}$ ) slip on exterior; very dark grey $(2.5 \mathrm{Y} 3 / 1)$ slip on interior. Hard, thin paste, non-porous, fine, grey (10YR 5/1) fabric with rare lime inclusion.

398. (No. 1208): Rim fragment; Kimistene, summit of the Acropolis, southern slope, found in 2005. pl. 16/398 and pl. 43/398.

Max. h 2.0 cm., d of rim 14.8 cm., max. w 4.8 cm., max. th $0.5 \mathrm{~cm}$.

Very dark grey $(2.5 \mathrm{Y} 3 / 1)$ slip on exterior and interior. Hard, non-porous, thin paste, fired to brown (7.5YR 5/3) and greyish brown (10YR $5 / 2$ ) fabric with some micaceous inclusion.

Bowl Form 2 (pl. 16, nos. 399-400)

Outcurved rim bowl.

399. (No. 424): Rim fragment; apsidial building, found in 2007. pl. 16/399 and pl. 43/399. Max. h $2.6 \mathrm{~cm}$., d of rim $11.0 \mathrm{~cm}$., max. w 3.5 cm., max. th $0.3 \mathrm{~cm}$.

Black (Gley $12.5 / \mathrm{N}$ ) slip on all of the surface. Hard, thin paste, non-porous, fine, light brown (7.5YR 6/4) fabric with no visible inclusion. 
400. (No. 157): Rim fragment; Domus, found in 2007. pl. 16/400 and pl. 43/400.

Max. h $2.1 \mathrm{~cm}$., d of rim $13.4 \mathrm{~cm}$., max. w 4.2 cm., max. th $0.3 \mathrm{~cm}$.

Dark grey (Gley $14 / \mathrm{N})$ slip on upper exterior; its below part and interior are slipped in grey (Gley $16 / \mathrm{N}$ ). Hard, thin paste, very sparsely porous, fine, grey (Gley $15 / \mathrm{N}$ ) fabric with no visible inclusion.

Dish Form 1 (pls. 16-17, nos. 401-404)

Dull slipped.

401. (No. 425): Rim fragment; apsidial building, found in 2007. pl. 16/401 and pl. 43/401.

Max. h $2.8 \mathrm{~cm}$., max. w $4.8 \mathrm{~cm}$., max. th 0.5 $\mathrm{cm}$.

Grey $(2.5 Y$ 5/1) slip on exterior and interior. Hard, non-porous, thin paste, fine, grey $(2.5 \mathrm{Y}$ 6/1) fabric with no visible inclusions.

402. (No. 158): Rim fragment; Domus, found in 2007. pl. 17/402 and pl. 43/402.

Max. h 3.4 cm., d of rim 15.5 cm., max. w 7.5 cm., max. th $0.4 \mathrm{~cm}$.

Grey (2.5Y 5/1) slip on exterior; dark grey (Gley $14 / \mathrm{N}$ ) slip on interior. Hard, thin paste, very sparsely porous, fine, grey (10YR 6/1) fabric with no visible inclusions.

403. (No. 58): Rim fragment; Domus, room 6, level 1, found in 2007. pl. 17/403 and pl. 43/403.

Max. h 3.9 cm., d of rim 20.8 cm., max. w 9.1 cm., max. th $0.6 \mathrm{~cm}$.

Dark grey (2.5Y 4/1) slip on exterior; grey (2.5Y 5/1) slip on interior. Hard, thin paste, very sparsely porous, fine, grey $(2.5 \mathrm{Y} 5 / 1)$ fabric with rare tiny lime inclusions.

404. (No. 251): Rim fragment; Western Tomb, found 2007. pl. 17/404 and pl. 43/404.
Max. h 4.4 cm., d of rim 25.0 cm., max. w 6.8 cm., max. th $0.5 \mathrm{~cm}$.

Dark grey (Gley $14 / \mathrm{N}$ ) slip on exterior; very dark grey (2.5Y 3/1) slip on interior. Hard, thin paste, very sparsely porous, fired to brown (10YR 4/3) and dark grey (10YR 4/1) fabric with rare tiny lime inclusions.

Dish Form 2 (pl. 17, nos. 405-409)

405. (No. 249): Rim fragment; western tomb, found 2007. pl. 17/405 and pl. 43/405.

Max. h $2.0 \mathrm{~cm}$., d of rim $10.2 \mathrm{~cm}$., max. w 5.3 cm., max. th $0.3 \mathrm{~cm}$.

Dark grey $(2.5 \mathrm{Y} 4 / 1)$ slip on exterior and interior. Average hardness; thin paste, very sparsely porous, fine, dark grey $(2.5 \mathrm{Y} 4 / 1)$ fabric with rare sand inclusions.

406. (No. 248): Rim fragment; western tomb, found in 2007. pl. 17/406 and pl. 43/406.

Max. h $3.0 \mathrm{~cm}$., d of rim $18.0 \mathrm{~cm}$., max. w 5.3 cm., max. th $0.6 \mathrm{~cm}$.

Black (Gley $12.5 / \mathrm{N}$ ) slip on the exterior; dark reddish grey (5YR 4/2) slip on the interior. Hard, thin paste, non-porous, fine, grey (10YR 5/1) and dark greyish brown (10YR $4 / 2$ ) fabric with rare tiny lime inclusion.

407. (No. 250): Rim fragment; Western Tomb, found 2007. pl. 17/407 and pl. 43/407.

Max. h 4.2 cm., d of rim 18.4 cm., max. w 3.4 cm., max. th $0.5 \mathrm{~cm}$.

Black (Gley 1 2.5/N) slip on exterior; very dark grey (Gley $13 / \mathrm{N}$ ) slip on interior. Hard, thin paste, non-porous, fine, dark grey (Gley $14 / \mathrm{N}$ ) fabric with occasional tiny lime inclusions.

408. (No. 354): Rim fragment; monumental tomb, found in 2007. pl. 17/408 and pl. 43/408.

Max. h 2.7 cm., d of rim $24.0 \mathrm{~cm}$., max. w 6.6 
cm., max. th $0.7 \mathrm{~cm}$.

Dark grey $(2.5 \mathrm{Y} 4 / 1)$ slip on exterior; very dark grey $(2.5 \mathrm{Y} 3 / 1)$ slip on interior. Hard, thin paste, very sparsely porous, fine, grey $(2.5 Y 5 / 1)$ fabric with rare tiny lime inclusions.

409. (No. 196): Rim fragment; Bath A, room 5, found in 2006. pl. 17/409 and pl. 43/409.

Max. h 2.7 cm., d of rim $24.8 \mathrm{~cm}$., max. w 5.8 cm., max. th $0.3 \mathrm{~cm}$.

Very dark grey (Gley $13 / \mathrm{N}$ ) slip on the exterior and interior. Hard, thin paste, very sparsely porous, fine, bluish grey (Gley 2 5/5PB) fabric with no visible inclusion.

Rim Fragment of a Plate (pl. 17, no. 410)

410. (No. 254): Rim fragment; Western Tomb, found 2007. pl. 17/410 and pl. 43/410.

Max. h $3.8 \mathrm{~cm}$., max. w $4.8 \mathrm{~cm}$., max. th 0.7 $\mathrm{cm}$.

Very dark grey (Gley $13 / \mathrm{N})$ slip on exterior; very dark grey $(2.5 \mathrm{Y} 3 / 1)$ slip on interior. Hard, thin paste, very sparsely porous, fine, grey (10YR 5/1) fabric with occasional sand inclusions. Exterio mottled.

Base Fragments of Open Forms (pl. 18, nos. 411-418)

Most of them has a shallow form. Their dimensions differ between 10.4 and $35.6 \mathrm{~cm}$.

411. (No. 216): Base fragment; Bath A, room 1, found 2006. pl. 18/411 and pl. 43/411.

Max. h $0.8 \mathrm{~cm}$., max. w $5.2 \mathrm{~cm}$., max. th 0.6 $\mathrm{cm}$.

Very dark grey (Gley $13 / \mathrm{N}$ ) slip on exterior and interior. Hard, thin paste, very sparsely porous, fine, grey (Gley $15 / \mathrm{N}$ ) fabric with some sand inclusions.
412. (No. 29): Base fragment; domus, room 1 , level 1, illegally excavated area, found in 2007. pl. 18/412 and pl. 43/412.

Max. h $2.5 \mathrm{~cm}$., d of base $10.4 \mathrm{~cm}$., max. w $3.2 \mathrm{~cm}$., max. th $0.7 \mathrm{~cm}$.

Greyish brown (10YR 5/2) slip on exterior; grey (10YR 5/1) slip on interior. Hard, thin paste, non-porous, fine, greyish brown (10YR $5 / 2$ ) fabric with rare tiny lime inclusions.

413. (No. 151): Base fragment; domus, found in 2007. pl. 18/ 413 and pl. 43/413.

Max. h $2.7 \mathrm{~cm}$., d of base $13.6 \mathrm{~cm}$., max. w $6.7 \mathrm{~cm}$., max. th $0.6 \mathrm{~cm}$.

Grey $(2.5 Y$ 5/1) and very dark grey (2.5Y 3/1) mottled slip on exterior; dark grey $(2.5 \mathrm{Y} 4 / 1)$ slip on interior. Hard, thin paste, non-porous, fine, grey $(2.5 \mathrm{Y} 5 / 1)$ fabric with rare tiny lime and micaceous inclusions.

414. (No. 3): Base fragment; domus, room 1, level 1, found in 2007. pl. 18/414 and pl. 44/414.

Max. h $2.0 \mathrm{~cm}$., d of base $30.6 \mathrm{~cm}$., max. w $4.5 \mathrm{~cm}$., max. th $0.6 \mathrm{~cm}$.

Very dark grey (Gley $13 / \mathrm{N}$ ) slip on exterior and interior. Hard, thin paste, very sparsely porous, fine, dark grey $(2.5 \mathrm{Y} 4 / 1)$ fabric with occasional tiny lime inclusions.

415. (No. 154): Base fragment; domus, found in 2007. pl. 18/415 and pl. 44/415.

Max. h 1.9 cm., d of base $17.0 \mathrm{~cm}$., max. w $4.6 \mathrm{~cm}$., max. th $0.8 \mathrm{~cm}$.

Very dark grey $(2.5 \mathrm{Y} 3 / 1)$ slip on exterior; dark grey $(2.5 \mathrm{Y} 4 / 1)$ slip on interior. Hard, thin paste, very sparsely porous, fine, grey $(2.5 \mathrm{Y} 5 / 1)$ fabric with rare tiny lime inclusions.

416. (No. 4): Base fragment; domus, room 1, level 1, found in 2007. pl. 18/416 and pl. 44/416. 
Max. h $2.3 \mathrm{~cm}$., d of base $35.6 \mathrm{~cm}$., max. w $5.5 \mathrm{~cm}$., max. th $0.7 \mathrm{~cm}$.

Dark grey (2.5Y 4/1) slip on exterior; grey (2.5Y 5/1) slip on interior. Hard, thin paste, very sparsely porous, fine, grey $(2.5 \mathrm{Y} 5 / 1)$ fabric with rare tiny lime inclusions.

417. (No. 5): Base fragment; domus, room 1, level 1, found in 2007. pl. 18/417 and pl. 44/417.

Max. h $3.1 \mathrm{~cm} ., \mathrm{d}$ of base $20.2 \mathrm{~cm}$. , max. w $2.3 \mathrm{~cm}$., max. th $0.3 \mathrm{~cm}$.

Dark grey $(2.5 \mathrm{Y} 4 / 1)$ slip on exterior; grey (2.5Y 5/1) slip on interior. Hard, thin paste, very sparsely porous, fine, grey $(2.5 \mathrm{Y} 5 / 1)$ fabric with rare tiny lime inclusions.

418. (No. 287): Base fragment; domus, room 6, level 1, found in 2007. pl. 18/418 and pl. 44/418.

Max. h $2.5 \mathrm{~cm}$., d of base $21.0 \mathrm{~cm}$., max. w $5.3 \mathrm{~cm}$., max. th $1.0 \mathrm{~cm}$.

Dark grey $(2.5 \mathrm{Y} 4 / 1)$ slip on exterior and interior. Hard, thin paste, non-porous, fine, grey (10YR 5/1) fabric with rare tiny lime inclusions.

Body Fragments of Open Forms (pl. 44, nos. 419-421)

419. (No. 54): Body fragment; domus, room 6, level 1, found in 2007. pl. 44/419.

Max. h $2.6 \mathrm{~cm}$., max. w $4.0 \mathrm{~cm}$., max. th 0.9 $\mathrm{cm}$.

Black (Gley $12.5 / \mathrm{N}$ ) slip on exterior and interior. Hard, thin paste, non-porous, fine, pale brown (10YR 6/3) fabric with no visible inclusions.

420. (No. 1378): Body fragment; Kepez, Necropolis, found 2005. pl. 44/420.

Max. h $4.1 \mathrm{~cm}$., max. w $3.8 \mathrm{~cm}$., max. th 0.5 $\mathrm{cm}$.
Light brownish grey (10YR 6/2) unslipped surface on exterior; black (Gley $12.5 / \mathrm{N}$ ) slip on interior. Hard, thin paste, non-porous, fired to brown (7.5YR 5/2) and very dark grey (Gley $13 / \mathrm{N}$ ) fabric with rare micaceous inclusions.

421. (No. 155): Body fragment; domus, found in 2007. pl. 44/421.

Max. h $3.6 \mathrm{~cm}$., max. w $7.5 \mathrm{~cm}$., max. th 0.7 $\mathrm{cm}$.

Dark grey $(2.5 \mathrm{Y} 4 / 1)$ and dark grey (Gley $14 / \mathrm{N})$ mottled slip on exterior and interior. Hard, thin paste, very sparsely porous, fine, dark grey $(2.5 \mathrm{Y} 4 / 1)$ fabric with no visible inclusions.

CLOSED FORMS (pls. 18-19, nos. 422436) / Rim Fragment of a Juglet (pl. 18, no. 422)

422. (No. 230): Rim fragment; Bath A, room 13, found 2006. pl. 18/422 and pl. 44/422.

Max. h $2.1 \mathrm{~cm}$., max. w $2.3 \mathrm{~cm}$., max. th 0.3 $\mathrm{cm}$.

Very dark grey (2.5Y 3/1) slip on exterior; dark grey $(2.5 \mathrm{Y} 4 / 1)$ slip on interior. Hard, thin paste, very sparsely porous, fired to grey (2.5Y 5/1) fabric with no visible inclusions.

Base Fragments of Closed Forms (pls. 18-19, nos. 423-435)

Small forms for some certain liquids. Some of them could be belonging to juglets or unguentaria.

423. (No. 68): Base fragment; domus, room 6, level 1, found in 2007. pl. 18/423 and pl. 44/423.

Max. h $2.3 \mathrm{~cm}$., d of base $2.6 \mathrm{~cm}$., max. w 4.3 cm., max. th $0.7 \mathrm{~cm}$.

Grey (2.5Y 6/1) unslipped surface on exterior and interior, Average hardness; thin paste, 
very sparsely porous, fine, grey $(2.5 \mathrm{Y} 5 / 1)$ fabric with no visible inclusions.

424. (No. 366): Base fragment; Bath B, room 8 , illegally excavated area, found in 2007. pl. 18/424 and pl. 44/424.

Max. h $1.8 \mathrm{~cm}$., d of base $2.8 \mathrm{~cm}$., max. w 3.5 cm., max. th $0.8 \mathrm{~cm}$.

Grey (2.5Y 6/1) slip on exterior; grey $(2.5 \mathrm{Y}$ $6 / 1$ ) unslipped surface on interior. Hard, thin paste, very sparsely porous, fine, grey $(2.5 \mathrm{Y}$ 5/1) fabric with no visible inclusions.

425. (No. 90): Base fragment; Bath B, Room 5, found in 2007. pl. 18/425 and pl. 44/425.

Max. h 1.4 cm., d of base $3.1 \mathrm{~cm}$., max. w 3.3 cm., max. th $0.6 \mathrm{~cm}$.

Grey (2.5Y 5/1) slip on exterior; grey $(2.5 \mathrm{Y}$ $5 / 1$ ) unslipped surface on interior. Hard, thin paste, non-porous, fine, dark grey (Gley 1 $4 / \mathrm{N}$ ) fabric with no visible inclusions.

426. (No. 241): Base fragment; western tomb, found 2007. pl. 18/426 and pl. 44/426.

Max. h $2.2 \mathrm{~cm}$., d of base $3.2 \mathrm{~cm}$., max. w 4.1 cm., max. th $0.8 \mathrm{~cm}$.

Very dark grey (Gley $13 / \mathrm{N})$ slip on exterior; grey $(2.5 \mathrm{Y} 6 / 1)$ unslipped surface on interior. Hard, thin paste, non-porous, fine, grey $(2.5 \mathrm{Y}$ 5/1) fabric with no visible inclusions.

427. (No. 92): Base fragment; Bath B, room 5, found in 2007. pl. 19/427 and pl. 44/427. Max. h $1.6 \mathrm{~cm}$., d of base $3.4 \mathrm{~cm}$., max. w 5.1 cm., max. th $0.6 \mathrm{~cm}$.

Dark grey $(2.5 \mathrm{Y} 4 / 1)$ unslipped surface on exterior, grey $(2.5 \mathrm{Y}$ 5/1) unslipped surface on interior, hard, thin paste, non-porous, fine, dark grey $(2.5 \mathrm{Y} 4 / 1)$ fabric with rare tiny lime inclusions.

428. (No. 107): Base fragment; Bath $B$, room 6, found in 2007. pl. 19/428 and pl. 44/428.
Max. h $1.7 \mathrm{~cm}$. , d of base $3.5 \mathrm{~cm}$., max. w 4.8 cm., max. th $0.6 \mathrm{~cm}$.

Dark grey $(2.5 \mathrm{Y} 4 / 1)$ slip on exterior, dark grey $(2.5 \mathrm{Y} 4 / 1)$ unslipped surface on interior, hard, thin paste, non-porous, fine, dark grey $(2.5 \mathrm{Y} 4 / 1)$ fabric with no visible inclusions.

429. (No. 252): Base fragment; western tomb, found 2007. pl. 19/429 and pl. 44/429.

Max. h $4.1 \mathrm{~cm}$., d of base $3.5 \mathrm{~cm}$., max. w 4.8 cm., max. th $0.7 \mathrm{~cm}$.

Very dark grey (Gley $13 / \mathrm{N})$ slip on exterior; dark grey (Gley $14 / \mathrm{N}$ ) unslipped surface on interior. Hard, thin paste, non-porous, fine, dark grey (Gley $14 / \mathrm{N}$ ) fabric with some tiny lime inclusions.

430. (No. 8): Base fragment; domus, room 1, level 1, found in 2007. pl. 19/430 and pl. 44/430.

Max. h $1.6 \mathrm{~cm}$., d of base $3.6 \mathrm{~cm}$., max. w 3.5 cm., max. th $0.8 \mathrm{~cm}$.

Grey (2.5Y 6/1) unslipped surface on exterior; grey (2.5Y 5/1) unslipped surface on interior. Hard, thin paste, very sparsely porous, fine, dark grey $(2.5 \mathrm{Y} 4 / 1)$ fabric with rare tiny lime inclusions.

431. (No. 36): Base fragment; domus, room 6, level 1, found in 2007. pl. 19/431 and pl. 44/431.

Max. h $2.0 \mathrm{~cm}$., d of base $3.6 \mathrm{~cm}$., max. w 5.0 cm., max. th $0.6 \mathrm{~cm}$.

Grey $(2.5 \mathrm{Y} 5 / 1)$ slip on exterior; grey $(2.5 \mathrm{Y}$ $6 / 1$ ) unslipped surface on interior. Hard, thin paste, very sparsely porous, fine, grey $(2.5 \mathrm{Y}$ $5 / 1$ ) fabric with rare micaceous inclusions.

432. (No. 106): Base fragment; Bath $B$, room 6, found in 2007. pl. 19/432 and pl. 44/432. Max. h $2.3 \mathrm{~cm}$., d of base $3.6 \mathrm{~cm}$., max. w 5.1 cm., max. th $0.7 \mathrm{~cm}$.

Very dark grey (Gley $13 / \mathrm{N})$ slip on exterior; 
very dark grey (Gley $13 / \mathrm{N})$ unslipped surface on interior. Hard, thin paste, non-porous, fine, black (Gley 1 2.5/N) fabric with some sand inclusions.

433. (No. 6): Base fragment; domus, room 1, level 1, found in 2007. pl. 19/433 and pl. 44/433.

Max. h $3.3 \mathrm{~cm}$., d of base $4.6 \mathrm{~cm}$., max. w 4.4 cm., max. th $1.0 \mathrm{~cm}$.

Grey (2.5Y 5/1) slip on exterior, grey (2.5Y $6 / 1$ ) unslipped surface on interior. Hard, thin paste, very sparsely porous, fine, dark grey $(2.5 \mathrm{Y} 4 / 1)$ fabric with rare micaceous inclusions.

434. (No. 37): Base fragment; domus, room 6, level 1, found in 2007. pl. 19/434 and pl. 44/434.

Max. h $2.8 \mathrm{~cm}$., d of base $5.4 \mathrm{~cm}$., max. w 5.5 cm., max. th $0.6 \mathrm{~cm}$.

Black (Gley $12.5 / \mathrm{N}$ ) slip on exterior; dark grey (Gley $14 / \mathrm{N}$ ) unslipped surface on exterior and interior. Hard, thin paste, non-porous, fine, dark grey (Gley $14 / \mathrm{N}$ ) fabric with some tiny lime inclusions.

435. (No. 174): Base fragment; domus, room 8, floor level, found in 2007. pl. 19/435 and pl. 44/435.

Max. h $1.8 \mathrm{~cm}$., d of base $5.4 \mathrm{~cm}$., max. w 4.5 cm., max. th $0.4 \mathrm{~cm}$.

Grey (10YR 6/1) unslipped surface on exterior; grey (2.5Y 6/1) unslipped surface on interior. Hard, thin paste, very sparsely porous, fine, grey (2.5Y 5/1) fabric with no visible inclusions.

Handle Fragment of Closed Form (pl. 19 , no. 436)

Rounded handle fragment of a small jug.
436. (No. 371): Handle fragment; Bath B, room 6 , illegally excavated area, found in 2007. pl. 19/436 and pl. 45/436.

Max. h 7.9 cm., max. w 3.7 cm., max. th 1.4 $\mathrm{cm}$.

Dark grey (2.5Y 4/1) and grey (2.5Y 5/1) mottled slip on all of surface. Hard, thin paste, very sparsely porous, fine, dark grey $(2.5 \mathrm{Y}$ $4 / 1$ ) fabric with rare tiny lime inclusions.

Body Fragments of Closed Forms (pl. 45, nos. 437-450)

437. (No. 214): Body fragment; Bath A, room 1, found in 2006. pl. 45/437.

Max. h $2.0 \mathrm{~cm}$., max. w $1.4 \mathrm{~cm}$., max. th 0.3 $\mathrm{cm}$.

Dark grey (Gley $14 / \mathrm{N}$ ) slip on exterior; grey (2.5Y 6/1) unslipped surface on interior. Average hardness; thin paste, non-porous, fine, dark grey $(2.5 \mathrm{Y} 4 / 1)$ fabric with no visible inclusions.

438. (No. 473): Body fragment; Bath A, room 2b, found in 2006. pl. 45/438.

Max. h $2.6 \mathrm{~cm}$., max. w $2.5 \mathrm{~cm}$., max. th 0.3 $\mathrm{cm}$.

Very dark grey (2.5Y 3/1) slip on exterior; grey $(2.5 Y 5 / 1)$ unslipped surface on interior. Hard, thin paste, very sparsely porous, fine, grey $(2.5 Y 5 / 1)$ fabric with no visible inclusions.

439. (No. 211): Body fragment; Bath A, room 1 , found in 2006. pl. 45/439.

Max. h 2.5 cm., max. w 2.9 cm., max. th 0.4 $\mathrm{cm}$.

Black (Gley 1 2.5/N) slip on exterior; very dark grey (Gley $13 / \mathrm{N}$ ) unslipped surface on interior. Hard, thin paste, non-porous, fine, very dark grey (Gley $13 / \mathrm{N}$ ) fabric with no visible inclusions. 
440. (No. 7): Body fragment; domus, room 1 , level 1, found in 2007. pl. 45/440.

Max. h $3.2 \mathrm{~cm}$., max. w $3.5 \mathrm{~cm}$., max. th 0.8 $\mathrm{cm}$.

Grey (2.5Y 6/1) slip on exterior; grey (2.5Y $6 / 1$ ) unslipped surface on interior. Hard, thin paste, very sparsely porous, fine, grey $(2.5 \mathrm{Y}$ $5 / 1$ ) fabric with some sand inclusions.

441. (No. 215): Body fragment; Bath A, room 1, found in 2006. pl. 45/441.

Max. h $2.3 \mathrm{~cm}$., max. w $2.9 \mathrm{~cm}$., max. th 0.5 $\mathrm{cm}$.

Black (2.5Y 2.5/1) slip on exterior; light grey (2.5Y 7/1) unslipped surface on interior. Hard, thin paste, non-porous, fine, grey (2.5Y 6/1) fabric with rare tiny lime inclusions.

442. (No. 180): Body fragment; domus, room 5, found in 2007. pl. 45/442.

Max. h $3.6 \mathrm{~cm}$., max. w $3.5 \mathrm{~cm}$., max. th 0.6 $\mathrm{cm}$.

Very dark grey (10YR 3/1) slip on exterior; grey $(2.5 Y$ 6/1) unslipped surface on interior. Hard, thin paste, very sparsely porous, fine, grey $(2.5 Y 5 / 1)$ fabric with rare tiny lime inclusions.

443. (No. 894): Body fragment; Kimistene, Acropolis, southern slope, underground cave, surface find, found in 2005. pl. 45/443.

Max. h $2.6 \mathrm{~cm}$., max. w $3.6 \mathrm{~cm}$., max. th 0.6 $\mathrm{cm}$.

Very dark grey (2.5Y 3/1) slip on exterior; grey $(2.5 Y$ 6/1) unslipped surface on interior. Hard, thin paste, non-porous, fine, grey (2.5Y 6/1) fabric with no visible inclusions.

444. (No. 281): Body fragment; Bath A, room 14, found in 2006. pl. 45/444.

Max. h 2.9 cm., max. w $4.1 \mathrm{~cm}$., max. th 0.5 $\mathrm{cm}$.

Black (2.5Y 2.5/1) slip on exterior; grey (2.5Y
5/1) unslipped surface on interior. Hard, thin paste, non-porous, fine, dark grey (2.5Y 4/1) fabric with occasional sand inclusions.

445. (No. 245): Body fragment; western tomb, found in 2007. pl. 45/445.

Max. h 4.4 cm., max. w $2.6 \mathrm{~cm}$., max. th 0.3 $\mathrm{cm}$.

Very dark grey (Gley $13 / \mathrm{N})$ slip on exterior; grey (2.5Y 6/1) unslipped surface on interior. Hard, thin paste, non-porous, fired to dark grey $(2.5 \mathrm{Y} 4 / 1)$ and grey $(2.5 \mathrm{Y} 5 / 1)$ fabric with no visible inclusions.

446. (No. 285): Body fragment; Bath A, room 14, found in 2006. pl. 45/446.

Max. h 2.7 cm., max. w $5.0 \mathrm{~cm}$., max. th 0.4 $\mathrm{cm}$.

Dark greyish brown $(2.5 \mathrm{Y} 4 / 2)$ slip on upper exterior; dark grey $(2.5 \mathrm{Y} 4 / 1)$ unslipped surface on interior. Hard, thin paste, very sparsely porous, fired to dark grey $(2.5 \mathrm{Y} 4 / 1)$ fabric with rare tiny lime inclusions.

447. (No. 217): Body fragment; Bath A, room 1, found in 2006. pl. 45/447.

Max. h $3.1 \mathrm{~cm}$., max. w 4.9 cm., max. th 0.6 $\mathrm{cm}$.

Dark grey $(2.5 \mathrm{Y} 4 / 1)$ slip on exterior; grey (2.5Y 6/1) unslipped surface on interior. Hard, thin paste, non-porous, fine, grey $(2.5 \mathrm{Y} 5 / 1)$ fabric with no visible inclusions.

448. (No. 547): Body fragment; surface find from the Village Boncuklar, found in 2005.

pl. 45/448.

Max. h 4.9 cm., max. w $5.0 \mathrm{~cm}$., max. th 0.5 $\mathrm{cm}$.

Black (Gley 1 2.5/N) slip on exterior; light brownish grey (10YR 6/2) unslipped surface on interior. Hard, thin paste, non-porous, fine, grey (10YR 5/1) fabric with no visible inclusions. 
449. (No. 164): Body fragment; domus, found in 2007. pl. 45/449.

Max. h $4.7 \mathrm{~cm}$., max. w $6.3 \mathrm{~cm}$., max. th 0.6 $\mathrm{cm}$.

Very dark grey (2.5Y 3/1) slip on exterior; grey $(2.5 \mathrm{Y} 6 / 1)$ unslipped surface on interior. Hard, thin paste, sparsely porous, fine, grey $(2.5 Y$ Y 6/1) fabric with occasional sand inclusions.

450. (No. 142): Body fragment; domus, room 4, found in 2007. pl. 45/450.

Max. h $1.9 \mathrm{~cm}$., max. w $4.5 \mathrm{~cm}$., max. th 1.4 $\mathrm{cm}$.

Dark grey (Gley $14 / \mathrm{N}$ ) slip on exterior; grey (2.5Y 6/1) unslipped surface on interior. Hard, thin paste, very sparsely porous, fine, grey (2.5Y 6/1) fabric with no visible inclusions.

\section{LATE HELLENISTIC-EARLY RO-} MAN BROWN-SLIPPED WARE (pls. 20-23, nos. 451-526)

Brown-slipped ware is a close relative of local sigillata in terms of fabric, shapes, slip techniques applied etc.: they are thin walled and consist of mostly open forms with brown and more matt slip. It is a hard, fine and non-porous fabric with less inclusions (sand, micaceous, lime and grit) in small sizes. Its production and firing technics are less careless than sigillata. Its paste is reddish yellow (5YR 6/6-7/6, 7.5YR 6/6-7/6), light brown (7.5YR 6/4), pink (5YR 7/4, 7.5YR 7/4), light yellowish brown (10YR 6/4, 2.5Y 6/3-6/4), red (2.5YR 5/6), brown (7.5YR 5/3-5/4), very pale brown (10YR 7/3-7/4), pale brown (10YR $6 / 3$ ), yellowish red (5YR 5/6), light reddish brown (5YR 6/4), reddish brown (5YR 5/4) and light red (2.5YR 6/8-6/6). Slip is brown (7.5YR 4/24/3-4/4-5/3-5/4, 10YR 4/3-5/3), reddish brown (2.5 YR 4/3-4/4-5/4, 5YR 4/3-4/4-5/3-5/4), red (2.5YR 5/6-4/6-4/8), dark brown (7.5YR 3/2-
3/3), black (7.5YR 2.5/1, 2.5Y 2.5/1), yellowish red (5YR 5/6), reddish yellow (5YR 6/6, 7.5YR 6/6-7/6), dark greyish brown (10YR 4/2), dark reddish brown (5YR 3/2), weak red (2.5YR 4/2), light brown (7.5YR 6/4), dark reddish grey (5YR $4 / 2)$, very dark grey (10YR 3/1, $2.5 \mathrm{Y} 3 / 1)$, pale brown (10YR 6/3, $2.5 \mathrm{Y} 7 / 4-8 / 4)$, dark grey (2.5Y 4/1), pink (5YR 7/4), strong brown (7.5YR 5/6), very dark greyish brown (10YR 3/2) and yellow (10YR 7/6). Because of bad firing technics surface slip is frequently mottled in brown (7.5YR 4/3-4/2), dark reddish brown (5YR 3/3-3/2, 2.5YR 3/3), reddish brown (2.5YR 4/4, 5YR 5/3$5 / 4)$, dark grey (10YR 4/1), strong brown (7.5YR $5 / 6$ ), dark reddish grey (5YR 4/2), very dark greyish brown (10YR 3/2), very dark grey (5YR 3/1), black (5YR 2.5/1, 10YR 2.5/1), red (2.5YR 5/6), dark greyish brown (10YR 4/2), reddish yellow (5YR 6/6), duksy red (2.5YR 3/2) and yellowish brown (10YR 5/4). Their bases left unslipped.

89 sherds were collected in total, 59 of which belong to the open and 30 to the closed forms. 22 of them were found in Bath A at Hadrianopolis, 17 in Kepez, 10 on the southern slope of the Acropolis at Kimistene and 3 in the western tomb at Hadrianopolis.

1 st century B.C.-1st century A.D.

OPEN FORMS (pls. 20-22, nos. 451-492)

Most common forms are bowls.

Bowl Form 1 (pl. 20, nos. 451-456)

Incurved rim bowls with mottled surface.

451. (No. 1413): Rim fragment; Kepez, surface find, found in 2005. pl. 20/451 and pl. 45/451.

Max. h 1.4 cm., max. w 1.9 cm., max. th 0.3 $\mathrm{cm}$.

Brown (7.5YR 5/3) slip on exterior; yellow- 
ish red (5YR 5/6) slip on interior. Hard, thin paste, non-porous, fine, reddish yellow (5YR 6/6) fabric with rare tiny lime and sand inclusions.

452. (No. 1104): Rim fragment; Kepez, found in 2005. pl. 20/452 and pl. 45/452.

Max. h $1.5 \mathrm{~cm}$., max. w $2.0 \mathrm{~cm}$., max. th 0.4 $\mathrm{cm}$.

Reddish yellow (7.5YR 6/6) and brown (7.5YR 4/3) mottled slip on exterior; reddish yellow (7.5YR 7/6) unslipped surface on interior. Hard, thin paste, very sparsely porous, fine, reddish yellow (7.5YR 6/6) fabric with frequent tiny lime inclusions.

453. (No. 901): Rim fragment; Kimistene, Acropolis, southern slope, underground cave, surface find, found in 2005. pl. 20/453 and pl. 45/453.

Max. h 1.8 cm., max. w $1.7 \mathrm{~cm}$., max. th 0.4 $\mathrm{cm}$.

Red (2.5YR 5/6) and reddish brown (2.5YR 4/4) mottled slip on exterior; reddish brown (5YR 4/3) and slip on interior. Hard, thin paste, non-porous, fine, reddish yellow (5YR 6/6) fabric with occasional sand and tiny lime inclusions.

454. (No. 1233): Rim fragment; Kepez, found in 2005. pl. 20/454 and pl. 45/454.

Max. h $2.0 \mathrm{~cm}$., max. w $3.4 \mathrm{~cm}$., max. th 0.5 $\mathrm{cm}$.

Weak red (2.5YR 4/2) slip on exterior and interior rim. Their below parts have red $(2.5 \mathrm{YR}$ 5/6) slip; hard, thin paste, non-porous, fine, red (2.5YR 5/6) fabric with rare medium sand inclusions.

455. (No. 343): Rim fragment; Bath B, Room 1, found in 2007. pl. 20/455 and pl. 45/455.

Max. h 2.2 cm., d of rim 11.5 cm., max. w 3.7 cm., max. th $0.4 \mathrm{~cm}$.
Dark brown (7.5YR 3/2) slip on exterior rim and interior. Its below part has reddish yellow (7.5YR 7/6) unslipped surface on exterior. Hard, thin paste, non-porous, fine, reddish yellow (7.5YR 6/6) fabric with some tiny lime inclusions.

456. (No. 931): Rim fragment; Kimistene, Acropolis, found in 2005. pl. 20/456 and pl. 45/456.

Max. h $2.6 \mathrm{~cm}$., d of rim $13.6 \mathrm{~cm}$., max. w 4.0 cm., max. th $0.5 \mathrm{~cm}$.

Brown (7.5YR 5/3) slip on exterior and interior. Hard, thin paste, non-porous, fine, brown (10YR 6/4) fabric with occasional sand inclusions.

Bowl Form 2 (pl. 20, nos. 463-468)

Outcurved rim bowl, sometimes with a groove on the rim.

457. (No. 297): Rim fragment; Bath A, Room 2b, found in 2006. pl. 45/457.

Max. h $0.8 \mathrm{~cm}$., max. w $1.3 \mathrm{~cm}$., max. th 0.3 $\mathrm{cm}$.

Brown (7.5YR 4/2) and pale brown (2.5Y 7/4) slip on exterior; red (2.5YR 4/6) slip on interior. Average hardness; thin paste, non-porous, fine, reddish yellow (7.5YR 7/6) fabric with no visible inclusions.

458. (No. 591): Rim fragment; Kimistene, Acropolis, southern slope, just below the summit, up to Deresemail creek, found in 2005. pl. 45/458.

Max. h $1.6 \mathrm{~cm}$., max. w $2.1 \mathrm{~cm}$., max. th 0.5 $\mathrm{cm}$.

Red (2.5YR 5/6) slip on exterior; light brown (7.5YR 6/4) slip on interior. Average hardness; thin paste, non-porous, fine, pink (7.5YR 7/4) fabric with rare sand and micaceous inclusions. 
459. (No. 584): Rim fragment; Kimistene, Acropolis, southern slope, just below the summit, up to Deresemail creek, found in 2005. pl. 45/459.

Max. h 1.6 cm., max. w $1.5 \mathrm{~cm}$., max. th 0.4 $\mathrm{cm}$.

Reddish brown (5YR 5/4) eroded slip on exterior and interior. Average hardness; thin paste, non-porous, fine, light brown (7.5YR 6/4) fabric with frequent tiny lime inclusions.

460. (No. 1060): Rim fragment; Kepez, found in 2005. pl. 45/460.

Max. h 2.3 cm., max. w 1.5 cm., max. th 0.4 $\mathrm{cm}$.

Reddish brown (5YR 5/4) mottled slip on exterior and interior. Hard, thin paste, non-porous, fine, very pale brown (10YR 7/4) fabric with some tiny lime and sand inclusions.

461. (No. 520): Rim fragment; Kimistene, Acropolis, southern slope, just below the summit, up to Deresemail creek, found in 2005. pl. 45/461.

Max. h 1.5 cm., max. w 2.2 cm., max. th 0.5 $\mathrm{cm}$.

Red (2.5YR 5/6) slip on exterior; dark reddish brown (5YR 3/3) and red (2.5YR 5/6) mottled slip on interior. Hard, thin paste, non-porous, fine, yellowish red (5YR 5/6) fabric with no visible inclusions.

462. (No. 1374): Rim fragment; Kimistene, Acropolis, southern slope, just below the summit, up to Deresemail creek, found in 2005. pl. 45/462.

Max. h 1.8 cm., max. w 1.7 cm., max. th 0.5 $\mathrm{cm}$.

Red (2.5YR 5/8) and dark reddish brown (2.5YR 3/3) mottled slip on exterior; reddish brown (5YR 4/3) slip on interior rim. Its below part is pink (5YR 7/4) unslipped surface on interior. Hard, thin paste, non-porous, fine, reddish yellow (5YR 6/6) fabric with no visible inclusions.

463. (No. 851): Rim fragment; Kimistene, surface find, found in 2005. pl. 20/463 and pl. 45/463.

Max. h $2.5 \mathrm{~cm}$., max. w $2.5 \mathrm{~cm}$., max. th $0.5 \mathrm{~cm}$. Brown (7.5YR 4/2) abrasion slip on exterior and interior. Average hardness; thin paste, very sparsely porous, fine, light brown (7.5YR 6/4) fabric with tiny lime inclusions.

464. (No. 1362): Rim fragment; Kimistene, Cistern, eastern slope, found in 2005. pl. 20/464 and pl. 45/464.

Max. h $2.6 \mathrm{~cm}$., max. w $2.7 \mathrm{~cm}$., max. th 0.5 $\mathrm{cm}$.

Yellowish red (5YR 5/6) slip on exterior and interior. Hard, thin paste, very sparsely porous, fine, reddish yellow (5YR 7/6) fabric with rare tiny lime inclusions.

465. (No. 952): Rim fragment; Kimistene, Acropolis, found in 2005. pl. 20/465 and pl. 45/465.

Max. h 1.4 cm., d of rim 13.4 cm., max. w 2.7 cm., max. th $0.3 \mathrm{~cm}$.

Dark grey (10YR 4/1) and brown (10YR 5/3) mottled slip on exterior; dark grey $(2.5 \mathrm{Y} 4 / 1)$ slip on interior. Hard, thin paste, non-porous, fired to greyish brown (10YR 5/2) fabric with no visible inclusions.

466. (No. 1102): Rim fragment; Kepez, found in 2005. pl. 20/466 and pl. 45/466.

Max. h $2.0 \mathrm{~cm}$., d of rim $15.6 \mathrm{~cm}$., max. w 3.0 cm., max. th $0.4 \mathrm{~cm}$.

Dark reddish grey (5YR 4/2) slip on exterior; reddish brown (5YR 4/3) slip on interior. Average hardness; thin paste, very sparsely porous, fine, very pale brown (10YR 7/4) fabric with rare micaceous, tiny lime and sand inclusions. 
467. (No. 411): Rim fragment; Kimistene, surface find, found in 2005. pl. 20/467 and pl. 45/467.

Max. h 1.9 cm., d of rim $17.0 \mathrm{~cm}$., max. w 2.5 cm., max. th $0.3 \mathrm{~cm}$.

Reddish yellow (7.5YR 6/6) slip on exterior; dark reddish grey (5YR 4/2) and light brown (7.5YR 6/4) slip on interior. Hard, thin paste, non-porous, pink (7.5YR 7/4) fabric with rare sand inclusions.

468. (No. 238): Rim fragment; Bath A, room 12, found in 2006. pl. 20/468 and pl. 45/468. Max. h $3.3 \mathrm{~cm}$., d of rim $27.0 \mathrm{~cm}$., max. w 6.8 cm., max. th $0.6 \mathrm{~cm}$.

Red (2.5YR 5/6) slip on exterior; strong brown (7.5YR 5/6) slip on interior. Hard, thin paste, very sparsely porous, fine, light brown (7.5YR 6/4) fabric with no visible inclusions.

Dish (pl. 20, nos. 469-471)

A dish form with a flattened rim.

469. (No. 356): Rim fragment; Western Tomb, found in 2007. pl. 20/469 and pl. 46/469. Max. h $4.0 \mathrm{~cm}$., max. w $4.6 \mathrm{~cm}$., max. th $0.7 \mathrm{~cm}$. Red (2.5YR 5/6) slip on exterior rim and interior; pink (5YR 7/4) slip on lower exterior. Hard, thin paste, non-porous, fine, red (2.5YR $5 / 6$ ) fabric with some lime inclusions.

470. (No. 422): Rim fragment; Apsidial Building, found in 2007. pl. 20/470 and pl. 46/470. Max. h $3.2 \mathrm{~cm}$., d of rim $29.6 \mathrm{~cm}$., max. w 4.2 cm., max. th $0.7 \mathrm{~cm}$.

Reddish brown (2.5YR 5/4) slip on exterior and interior. Hard, thin paste, non-porous, fine, light red (2.5YR 6/8) fabric with some lime inclusions.

471. (No. 222): Rim fragment; Bath A, room 13, found in 2006. pl. 20/471 and pl. 46/471.
Max. h 2.7 cm., d of rim 15.4 cm., max. w 4.1 cm., max. th $0.5 \mathrm{~cm}$.

Brown (10YR 4/3) slip on exterior; very dark grey (10YR 3/1) slip on interior. Hard, thin paste, very sparsely porous, fine, light brown (7.5YR 6/4) fabric with no visible inclusions.

Other Rim Fragments (pl. 21, nos. 473475)

Some of them are thickened rim forms; some have mottled slips.

472. (No. 834): Rim fragment; Kimistene, Cistern, eastern slope, found in 2005. pl. 46/472.

Max. h $2.0 \mathrm{~cm}$., max. w $4.1 \mathrm{~cm}$., max. th 0.4 $\mathrm{cm}$.

Red (2.5YR 5/6) slip on exterior; red (2.5YR 4/6) slip on interior. Hard, thin paste, nonporous, fine, reddish yellow (5YR 6/6) fabric with no visible inclusions.

473. (No. 227): Rim fragment; Bath A, room 13, found in 2006. pl. 21/473 and pl. 46/473. Max. h $4.4 \mathrm{~cm}$., max. w $6.5 \mathrm{~cm}$., max. th 0.5 $\mathrm{cm}$.

Brown (7.5YR 4/3) and very dark greyish brown (10YR 3/2) mottled slip exterior and interior. Hard, thin paste, non-porous, fired to very dark grey (Gley $13 / \mathrm{N}$ ) fabric with some tiny lime inclusions.

474. (No. 242): Rim fragment; Western Tomb, found in 2007. pl. 21/474 and pl. 46/474.

Max. h $3.6 \mathrm{~cm}$., d of rim $18.8 \mathrm{~cm}$., max. w 5.4 cm., max. th $0.4 \mathrm{~cm}$.

Brown (7.5YR 4/2-4/3) slip on exterior; brown $(7.5 \mathrm{YR} 4 / 2)$ slip on interior. Average hardness; thin paste, non-porous, fine, light brown (7.5YR 6/4) fabric with no visible inclusions. 
475. (No. 1259): Rim fragment; Kimistene, Necropolis, surface find, found in 2005. pl. 21/475 and pl. 46/475.

Max. h $3.0 \mathrm{~cm}$., d of rim $24.0 \mathrm{~cm}$. , max. w 4.8 $\mathrm{cm} .$, max. th $0.6 \mathrm{~cm}$.

Reddish brown (5YR 4/4) slip on exterior and interior. Hard, thin paste, non-porous, fine, red $(2.5$ YR $5 / 6)$ fabric with frequent tiny lime inclusions.

Base Fragments of Open Forms (pls. 2122, nos. 478-492)

High based fragments; exterior surfaces left unslipped. Traces of slips are mottled.

476. (No. 373): Base fragment; Bath A, room $2 \mathrm{~b}$, found in 2006. pl. 46/476.

Max. h 1.3 cm., max. w $1.2 \mathrm{~cm}$., max. th 0.2 $\mathrm{cm}$.

Pink (5YR 7/4) unslipped surface on exterior; brown (7.5YR 4/3) slip on interior. Average hardness; thin paste, non-porous, fired to reddish yellow (5YR 6/6) and light brown (7.5YR 6/4) fabric with no visible inclusions.

477. (No. 372): Base fragment; Bath A, room $2 b$, found in 2006. pl. 46/476.

Max. h 1.3 cm., max. w $1.2 \mathrm{~cm}$., max. th 0.2 $\mathrm{cm}$.

Pink (7.5YR 7/4) unslipped surface on exterior; brown (7.5YR 4/3) slip on interior. Average hardness; thin paste, non-porous, fired to reddish yellow (5YR 6/6) and light brown (7.5YR 6/4) fabric with no visible inclusions.

478. (No. 529): Base fragment; Kimistene, Acropolis, southern slope, just below the summit, up to Deresemail creek, found in 2005. pl. 21/478 and pl. 46/478.

Max. h 1.5 cm., max. w 1.8 cm., max. th 0.4 $\mathrm{cm}$.

Reddish yellow (5YR 6/6) and reddish brown
(5YR 5/4) mottled slip on exterior; brown (7.5YR 4/3) slip on interior. Hard, thin paste, non-porous, fine, pink (7.5YR 7/4) fabric with no visible inclusions.

479. (No. 984): Base fragment; Hadrianopolis, surface find, found in 2005. pl. 21/479 and pl. 46/479.

Max. h 1.4 cm., max. w $3.0 \mathrm{~cm}$., max. th 0.5 $\mathrm{cm}$.

Pink (7.5YR 7/4) unslipped surface on exterior; reddish brown (2.5YR 4/4) slip on interior. Hard, thin paste, non-porous, fired to very pale brown (10YR 7/4) and pink (7.5YR 7/4) fabric with no visible inclusions.

480. (No. 95): Base fragment; Bath B, room 5, found in 2007. pl. 21/480 and pl. 46/480.

Max. h $1.6 \mathrm{~cm}$., d of base $2.8 \mathrm{~cm}$., max. w 3.0 cm., max. th $0.4 \mathrm{~cm}$.

Reddish brown (5YR 4/4) slip on exterior; dark reddish brown (5YR 3/2) slip on interior. Hard, thin paste, non-porous, fine, red (2.5YR $5 / 6$ ) fabric with no visible inclusions.

481. (No. 293): Base fragment; Bath A, room 2b, found in 2006. pl. 21/481 and pl. 46/481. Max. h $0.7 \mathrm{~cm}$., d of base $4.0 \mathrm{~cm}$., max. w 2.9 cm., max. th $0.3 \mathrm{~cm}$.

Pink (7.5YR 7/4) unslipped surface on exterior; brown (7.5YR 5/4) slip on interior. Hard, thin paste, very sparsely porous, fine, pink (7.5YR 7/4) fabric with no visible inclusions.

482. (No. 367): Base fragment; Bath B, room 8 , from the illegally excavated area in the southeast, found in 2007. pl. 21/482 and pl. 46/482.

Max. h $1.2 \mathrm{~cm}$., d of base $4.4 \mathrm{~cm}$., max. w 2.7 cm., max. th $0.4 \mathrm{~cm}$.

Pink (7.5YR 7/4) unslipped surface on exterior; reddish brown (5YR 5/4) slip on interior. Hard, thin paste, very sparsely porous, fine, 
light reddish brown (5YR 6/4) fabric with some tiny lime inclusions.

483. (No. 519): Base fragment; Kimistene, Acropolis, southern slope, just below the summit, up to Deresemail creek, found in 2005. pl. 21/483 and pl. 46/483.

Max. h $1.1 \mathrm{~cm}$., d of base $4.6 \mathrm{~cm}$., max. w 4.6 cm., max. th $0.3 \mathrm{~cm}$.

Pink (7.5YR 7/4) unslipped surface on exterior; brown (7.5YR 5/4) and very dark greyish brown (10YR 3/2) mottled slip on interior. Hard, thin paste, very sparsely porous, fine, reddish yellow (5YR 6/6) fabric with occasional tiny lime inclusions.

484. (No. 284): Base fragment; Bath A, room 14, found in 2007. pl. 21/484 and pl. 46/484. Max. h $1.2 \mathrm{~cm}$., d of base $5.2 \mathrm{~cm}$., max. w 5.4 cm., max. th $0.4 \mathrm{~cm}$.

Reddish yellow (5YR 6/6) and brown (7.5YR $4 / 2$ ) mottled slip on exterior; brown (10YR 5/3) and reddish yellow (5YR 6/6) mottled slip on interior. Average hardness; thin paste, very sparsely porous, fine, reddish yellow (5YR 6/6) fabric with infrequent lime, sand and occasional micaceous inclusions.

485. (No. 206): Base fragment; Bath A, room 1, found in 2006. pl. 21/485 and pl. 46/485.

Max. h $1.5 \mathrm{~cm}$., d of base $5.8 \mathrm{~cm}$., max. w 4.2 cm., max. th $0.4 \mathrm{~cm}$.

Light yellowish brown (2.5Y 6/3) unslipped surface on exterior; brown (10YR 5/3) slip on interior. Hard, thin paste, very sparsely porous, fine, light yellowish brown (2.5Y 6/4) fabric with occasional sand inclusions.

486. (No. 279): Base fragment; Bath A, room 14, found in 2007. pl. 21/486 and pl. 46/486. Max. h $1.6 \mathrm{~cm}$., d of base $6.8 \mathrm{~cm}$., max. w 4.6 cm., max. th $0.6 \mathrm{~cm}$.

Dark greyish brown (10YR 4/2) and brown
(10YR 4/3) mottled slip on exterior; dark greyish brown (10YR 4/2) slip on interior. Hard, thin paste, non-porous, fine, light brown (7.5YR 6/4) fabric with no visible inclusions.

487. (No. 355): Base fragment; Monumental Tomb, found in 2007. pl. 21/487 and pl. 46/487.

Max. h $1.3 \mathrm{~cm}$.,d of base $7.2 \mathrm{~cm}$., max. w 2.5 cm., max. th $0.5 \mathrm{~cm}$.

Pink (7.5YR 7/4) unslipped surface on exterior; reddish brown (5YR 5/4) slip on interior. Hard, thin paste, very sparsely porous, fine, pink (7.5YR 7/4) fabric with occasional micaceous inclusions.

488. (No. 253): Base fragment; Western Tomb, found in 2007. pl. 21/488 and pl. 46/488.

Max. h $1.6 \mathrm{~cm}$., d of base $8.8 \mathrm{~cm}$., max. w 8.2 cm., max. th $0.6 \mathrm{~cm}$.

Pink (5YR 7/4) unslipped surface on the exterior; reddish brown (5YR 4/3) slip in the interior. Hard, thin paste; very sparsely porous, fine, pink (5YR 7/4) fabric with tiny lime inclusion.

489. (No. 1384): Base fragment; surface find from Göletarkas1, found in 2005. pl. 22/489 and pl. 46/489.

Max. h $2.6 \mathrm{~cm}$., d of base $13.6 \mathrm{~cm}$., max. w $6.8 \mathrm{~cm}$., max. th $1.0 \mathrm{~cm}$.

Reddish brown (5YR 5/4) slip on exterior and interior. Exterior surface is burnished. Hard, thin paste, very sparsely porous, fine, reddish brown (5YR 5/4) fabric with some sand inclusions.

490. (No. 1469): Base fragment; surface find from the Village Boncuklar, found in 2005. pl. 22/490 and pl. 46/490.

Max. h $1.7 \mathrm{~cm}$., d of base $14.6 \mathrm{~cm}$., max. w $4.2 \mathrm{~cm}$., max. th $0.7 \mathrm{~cm}$.

Pale brown (10YR 6/3) slip on exterior; red- 
dish brown (5YR 4/3) slip on interior. Exterior surface is burnished. Hard, thin paste, nonporous, fired to grey $(2.5 \mathrm{Y} 5 / 1)$ fabric with sand and occasional micaceous inclusions.

491. (No. 932): Base fragment; Kimistene, Acropolis, found in 2005. pl. 22/491 and pl. 46/491.

Max. h $1.6 \mathrm{~cm}$., d of base $14.8 \mathrm{~cm}$., max. w $4.3 \mathrm{~cm}$., max. th $0.7 \mathrm{~cm}$.

Red (2.5YR 4/8) slip on exterior; brown (7.5YR 4/4) slip on interior. Three thin bands in very dark grey (7.5YR 3/1) on interior. Hard, thin paste, non-porous, fine, reddish brown (5YR 5/4) fabric with no visible inclusions.

492. (No. 650): Base fragment; Kimistene, Cistern, found in 2005. pl. 22/492 and pl. 46/492.

Max. h $4.0 \mathrm{~cm}$., d of base $18.0 \mathrm{~cm}$., max. w $6.8 \mathrm{~cm}$., max. th $0.7 \mathrm{~cm}$.

Light brown (7.5YR 6/4) slip on exterior; reddish brown (5YR 4/3) slip on interior. Hard, thin paste, non-porous, fine, yellowish red (5YR 5/6) fabric with some sand inclusion.

Body Fragments of Open Forms (pl. 47, nos. 493-509)

Fragments mostly with mottled slip.

493. (No. 1144): Body fragment; Kimistene, Acropolis, found in 2005. pl. 47/493.

Max. h 1.0 cm., max. w $1.4 \mathrm{~cm}$., max. th 0.3 $\mathrm{cm}$.

Black (2.5Y 2.5/1) slip on exterior; reddish brown (2.5YR 4/4) slip on interior. Hard, thin paste, non-porous, fine, pale brown (10YR 6/3) fabric with no visible inclusions.

494. (No. 1139): Body fragment; Kimistene, Acropolis, found in 2005. pl. 47/494.

Max. h $1.5 \mathrm{~cm}$., max. w $1.6 \mathrm{~cm}$., max. th $0.5 \mathrm{~cm}$.
Light brownish grey (2.5Y 6/2) unslipped surface on exterior; reddish brown (2.5YR 4/4) slip on interior. Hard, thin paste, non-porous, fine, light red (2.5YR 6/6) fabric with rare sand inclusions.

495. (No. 1141): Body fragment; Kimistene, Acropolis, found in 2005. pl. 47/495.

Max. h 1.9 cm., max. w 1.7 cm., max. th 0.4 $\mathrm{cm}$.

Reddish brown (2.5YR 4/4) and red (2.5YR $5 / 6)$ mottled slip on exterior; reddish brown (2.5YR 4/4) slip on interior. Hard, thin paste, non-porous, fine, pink (5YR 7/4) fabric with no visible inclusions.

496. (No. 522): Body fragment; Kepez, found in 2005. pl. 47/496.

Max. h $2.1 \mathrm{~cm}$., max. w $2.4 \mathrm{~cm}$., max. th 0.5 $\mathrm{cm}$.

Red (2.5YR 5/6) and reddish brown (2.5YR $5 / 4$ ) slip on exterior; reddish yellow (5YR 6/6) slip on interior. Hard, thin paste, sparsely porous, fine, reddish yellow (5YR 6/6) fabric with no visible inclusions.

497. (No. 1194): Body fragment; Kepez, surface find in the Cistern, found in 2005. pl. 47/497.

Max. h $1.5 \mathrm{~cm}$., max. w $3.0 \mathrm{~cm}$., max. th 0.5 $\mathrm{cm}$.

Yellowish red (5YR 5/6) slip on exterior; brown $(7.5 \mathrm{YR} 5 / 3)$ slip on interior. Exterior surface is burnished. Hard, thin paste, nonporous, fired to pale brown (10YR 6/3) and reddish brown (5YR 5/4) fabric with sand and rare micaceous inclusions.

498. (No. 1039): Body fragment; Kepez, found in 2005. pl. 47/498.

Max. h $2.3 \mathrm{~cm}$., max. w $2.3 \mathrm{~cm}$., max. th 0.4 $\mathrm{cm}$.

Red (2.5YR 4/6) slip on exterior; reddish 
brown (2.5YR 4/4) slip on interior. Hard, thin paste, very sparsely porous, fine, light reddish brown (5YR 6/4) fabric with frequent tiny lime inclusions.

499. (No. 1037): Body fragment; Kepez, found 2005. pl. 47/499.

Max. h $2.4 \mathrm{~cm}$., max. w $2.4 \mathrm{~cm}$., max. th 0.5 $\mathrm{cm}$.

Very dark grey (5YR 3/1) slip on exterior; red (2.5YR 5/6) slip on interior. Soft, thin paste, very sparsely porous, fine, brown (7.5YR 5/4) fabric with occasional lime inclusions.

500. (No. 693): Body fragment; Kimistene, Acropolis, southern slope, just below the summit, up to Deresemail creek, found in 2005. pl. 47/500.

Max. h $2.6 \mathrm{~cm}$., max. w $2.5 \mathrm{~cm}$., max. th 0.5 $\mathrm{cm}$.

Dusky red (2.5YR 3/2) and red (2.5YR $4 / 6)$ mottled slip on exterior; reddish brown (2.5YR 4/3) slip on interior. Hard, thin paste, very sparsely porous, fine, reddish yellow (5YR 6/6) fabric with infrequent lime and sand inclusions.

501. (No. 33): Body fragment; Domus, room 2, found in 2007. pl. 47/501.

Max. h $3.0 \mathrm{~cm}$., max. w $2.5 \mathrm{~cm}$., max. th 0.5 $\mathrm{cm}$.

Weak red (2.5YR 4/2) slip on exterior and interior. Hard, thin paste, very sparsely porous, fired to light brown (7.5YR 6/3-6/4) mottled fabric with infrequent lime inclusions.

502. (No. 1041): Body fragment; Kepez, found in 2005. pl. 47/502.

Max. h 2.7 cm., max. w 2.9 cm., max. th 0.6 $\mathrm{cm}$.

Dark greyish brown (10YR 4/2) and yellowish brown (10YR 5/4) mottled slip on exterior. Brown (10YR 4/3) slip on interior. Hard, thin paste, sparsely porous, fired to light yellowish brown (10YR 6/4) fabric with no visible inclusions.

503. (No. 570): Body fragment; surface find from the Village Boncuklar, found in 2005. pl. 47/503.

Max. h 3.4 cm., max. w $1.5 \mathrm{~cm}$., max. th 0.5 $\mathrm{cm}$.

Dark brown (7.5YR 4/2) slip on exterior; black (7.5YR 2.5/1) slip on interior. Hard, thin paste, non-porous, fine, light brown (7.5YR 6/4) fabric with rare sand inclusions.

504. (No. 1377): Body fragment; Kepez, Necropolis, found in 2005. pl. 47/504.

Max. h $3.1 \mathrm{~cm}$., max. w $3.2 \mathrm{~cm}$., max. th 0.7 $\mathrm{cm}$.

Dark brown (7.5YR 3/2) slip on exterior; reddish brown (5YR 4/4) slip on interior. Slip is shiny on all of surface. Hard, thin paste, nonporous, fine, reddish yellow (7.5YR 6/6) fabric with frequent tiny lime inclusions.

505. (No. 435): Body fragment; Kimistene, Acropolis, slope, found in 2005. pl. 47/505.

Max. h $2.7 \mathrm{~cm}$., max. w $4.5 \mathrm{~cm}$., max. th $0.7 \mathrm{~cm}$. Pink (7.5YR 7/4) unslipped surface on exterior; brown (7.5YR 4/3) slip on interior. Average hardness; thin paste, non-porous, fine, reddish yellow (7.5YR 6/6) fabric with no visible inclusions.

506. (No. 1410): Body fragment; Kepez, surface find, found in 2005. pl. 47/506.

Max. h 4.3 cm., max. w 4.3 cm., max. th 0.6 $\mathrm{cm}$.

Dark reddish grey (5YR 4/2) slip on exterior; reddish brown (5YR 5/4) slip on interior. Hard, very sparsely porous, thin paste, fired to light red (2.5YR 6/6) and reddish yellow (7.5YR 6/6) mottled fabric with some tiny lime inclusions. 
507. (No. 1501): Body fragment; Kepez, found in 2005. pl. 47/507.

Nos. 507 and 508 belong to each other.

Max. h $2.7 \mathrm{~cm}$., max. w $4.3 \mathrm{~cm}$., max. th $0.5 \mathrm{~cm}$. Very pale brown (10YR 8/3) unslipped surface on upper exterior; its below part is dark greyish brown (10YR 4/2) slip on exterior. Red (2.5YR 5/6) slip on interior. Hard, thin paste, very sparsely porous, fine, very pale brown (10YR 7/3) fabric with some tiny lime inclusions.

508. (No. 1044): Body fragment; Kepez, found in 2005. pl. 47/508.

Max. h $3.3 \mathrm{~cm}$., max. w $3.5 \mathrm{~cm}$., max. th $0.5 \mathrm{~cm}$. Very pale brown (10YR 8/3) unslipped surface on upper exterior; its below part is dark greyish brown (10YR 4/2) slip on exterior. Red (2.5YR 5/6) slip on interior. Hard, thin paste, very sparsely porous, fine, very pale brown (10YR 7/3) fabric with some tiny lime inclusions.

509. (No. 162): Body fragment; Domus, found in 2007. pl. 47/509.

Max. h 5.0 cm., max. w 4.9 cm., max. th 1.2 $\mathrm{cm}$.

Brown (7.5YR 5/3) slip on exterior; dark brown (7.5YR 3/2) slip on interior. Hard, nonporous, thin paste, fine, brown (7.5YR 5/3) fabric with occasional tiny lime inclusions.

CLOSED FORMS (pls. 22-23, nos. 510526) / Juglet (pl. 22, nos. 510-512)

Mostly thin-walled forms with blackish or mottled slip.

510. (No. 205): Rim fragment; Bath $A$, room 1, found in 2006. pl. 22/510 and pl. 47/510. Max. h 2.2 cm., d of rim 5.2 cm., max. w 2.9 cm., max. th $0.2 \mathrm{~cm}$.
It may has belonged to a kind of small a juglet with splayed mouth. Black (7.5YR 2.5/1) slip on exterior; reddish brown (5YR 5/3) slip on interior. Hard, non-porous, thin paste, fine, reddish yellow (5YR 6/6) fabric with no visible inclusions.

511. (No. 192): Rim fragment; Bath A, room 2, found in 2006. pl. 22/511 and pl. 47/511. Max. h 5.1 cm., d of rim 8.0 cm., max. w 7.3 cm., max. th $0.3 \mathrm{~cm}$.

Black (7.5YR 2.5/1) slip on exterior; dark brown (7.5YR 3/2) slip on interior. Pink (7.5YR 7/4) unslipped surface on lower interior. Hard, non-porous, thin paste, fine, pink (7.5YR 7/4) fabric with no visible inclusions. Parallel: Stewart 2010, 210, 304, fig. 244, 392 (P 2136) - a Middle Hellenistic pitcher from Gordion with an offset rim, long inset neck and sharply biconical wall (d. of rim $6.8 \mathrm{~cm}$ ).

512. (No. 1178): Rim fragment; Kimistene, Acropolis, southern slope, just below the summit, up to Deresemail creek, found in 2005. pl. 22/512 and pl. 47/512.

Max. h 2.9 cm., d of rim 10.4 cm., max. w 3.2 cm., max. th $0.6 \mathrm{~cm}$.

Very dark grey (10YR 3/1) slip on exterior rim; yellow (10YR 7/6) slip on lower exterior. Pale brown (2.5Y 8/4) slip and very dark grey $(2.5 \mathrm{Y} 3 / 1)$ thin band on lip. Black (10YR 2.5/1) and very dark greyish brown (10YR $3 / 2$ ) mottled slip on interior rim. Reddish yellow (7.5YR 6/6) unslipped surface on lower interior. Hard, thin paste, non-porous, fine, reddish yellow (7.5YR 6/6) fabric with no visible inclusions.

Base Fragments of Closed Forms (pls. 22-23, nos. 514-519)

Mostly unslipped, high bases. 
513. (No. 1489): Base fragment; surface find from the Village Boncuklar, found in 2005. pl. 47/513.

Max. h $1.3 \mathrm{~cm}$., max. w $1.8 \mathrm{~cm}$., max. th $0.3 \mathrm{~cm}$. Pink (7.5YR 8/4) unslipped surface on exterior and interior. Average hardness; thin paste, non-porous, fine, reddish yellow (5YR 7/6) fabric with no visible inclusions.

514. (No. 1201): Base fragment; Kepez, surface find in the Cistern, found in 2005. pl. 22/514 and pl. 47/514.

Max. h 2.5 cm., d of rim 4.4 cm., max. w 5.0 cm., max. th $0.5 \mathrm{~cm}$.

Reddish yellow (7.5YR 7/6) unslipped surface on exterior and interior. Hard, thin paste, non-porous, fired to reddish yellow (5YR 6/6) and pale brown (10YR 6/3) fabric with occasional micaceous inclusions.

515. (No. 1492): Base fragment; surface find from the Village Boncuklar, found in 2005. pl. 23/515 and pl. 47/515.

Max. h $1.6 \mathrm{~cm}$., d of base $5.0 \mathrm{~cm}$., max. w 3.8 cm., max. th $0.4 \mathrm{~cm}$.

Light brownish grey (10YR 6/2) unslipped surface on exterior and interior. Hard, thin paste, very sparsely porous, fired to grey $(2.5 \mathrm{Y} 5 / 1)$ fabric with occasional tiny lime inclusions.

516. (No. 946): Base fragment; Kimistene, Acropolis, found in 2005. pl. 23/516 and pl. 47/516.

Max. h $1.1 \mathrm{~cm}$., d of base $5.8 \mathrm{~cm}$., max. w 4.5 cm., max. th $0.3 \mathrm{~cm}$.

Brown (7.5YR 4/2) eroded slip on exterior; pink (5YR 7/4) unslipped surface on interior. Hard, thin paste, non-porous, fine, pink (5YR 7/4) fabric with no visible inclusions.

517. (No. 948): Base fragment; Kimistene, Acropolis, found in 2005. pl. 23/517 and pl.
47/517.

Max. h $1.4 \mathrm{~cm}$., d of base $6.0 \mathrm{~cm}$., max. w 4.9 cm., max. th $0.4 \mathrm{~cm}$.

Reddish yellow (5YR 6/6) unslipped surface on exterior; reddish yellow (7.5YR 7/6) unslipped surface on interior. Hard, thin paste, very sparsely porous, fired to reddish yellow (5YR 6/6) and (7.5YR 6/6) mottled fabric with some tiny lime inclusions.

518. (No. 1420): Base fragment; Church, found in 2005. pl. 23/518 and pl. 47/518.

Max. h $2.2 \mathrm{~cm}$., d of base $8.6 \mathrm{~cm}$., max. w 7.3 cm., max. th $0.8 \mathrm{~cm}$.

Red (2.5YR 5/6) slip on exterior; pink (5YR $7 / 4$ ) unslipped surface on interior. Hard, thin paste, very sparsely porous, fired to pink (5YR 7/4) and light reddish brown (2.5YR $6 / 4$ ) fabric with no visible inclusions.

519. (No. 787): Base fragment; Kimistene, Acropolis, southern slope, just below the summit, up to Deresemail creek, found in 2005. pl. 23/519 and pl. 47/519.

Max. h $3.5 \mathrm{~cm}$., d of base $11.4 \mathrm{~cm}$., max. w 3.1 cm., max. th $0.9 \mathrm{~cm}$.

Reddish brown (5YR 4/3) slip on exterior; pink (7.5YR 7/4) unslipped surface on interior. Hard, thin paste, non-porous, fired to reddish yellow (7.5YR 6/6) and (5YR 6/6) fabric with frequent tiny lime inclusions.

Handle Fragments of Closed Forms (pl. 23 , nos. 525-526)

Vertical handles with mottled surfaces.

520. (No. 1134): Handle fragment; Kimistene, Acropolis, found in 2005. pl. 48/520.

Max. h $1.6 \mathrm{~cm}$., max. w $1.3 \mathrm{~cm}$., max. th 0.7 $\mathrm{cm}$.

Dark reddish grey (5YR 3/2) and black (5YR 2.5/1) mottled slip on exterior and interior. 
Average hardness; thin paste, non-porous, fine, reddish yellow (5YR 6/6) fabric with occasional tiny lime inclusions.

521. (No. 412): Handle fragment; Hadrianopolis, surface find, found in 2005. pl. 48/521.

Max. h $1.5 \mathrm{~cm}$., max. w $1.4 \mathrm{~cm}$., max. th 0.6 $\mathrm{cm}$.

Red (2.5YR 5/6) and very dark grey (5YR $3 / 1$ ) mottled slip on exterior and interior. Hard, thin paste, non-porous, fine, reddish yellow (5YR 6/6) fabric with no visible inclusions.

522. (No. 240): Handle fragment; Bath A, room 12, found in 2006. pl. 48/522.

Max. h $2.0 \mathrm{~cm}$., max. w $1.7 \mathrm{~cm}$., max. th 0.6 cm.

Red (2.5YR 5/6) and dark reddish brown (5YR 3/2) mottled slip on exterior and interior. Average hardness; thin paste, very sparsely porous, fine, reddish yellow (5YR 6/6) fabric with no visible inclusions.

523. (No. 201): Handle fragment; Bath A, room 13a, below the floor, found in 2006. pl. 48/523.

Max. h $1.0 \mathrm{~cm}$., max. w $2.3 \mathrm{~cm}$., max. th 0.6 $\mathrm{cm}$.

Reddish brown (5YR 4/4) and very dark grey (5YR 3/1) mottled slip on exterior and interior. Hard, thin paste, non-porous, fired to reddish yellow (5YR 6/6) and light red (2.5YR 6/6) mottled fabric with rare micaceous and tiny lime inclusions.

524. (No. 224): Handle fragment; Bath A, Room 13, found in 2006. pl. 48/524.

Max. h 2.5 cm., max. w 1.2 cm., max. th 0.6 $\mathrm{cm}$.

Black (7.5YR 2.5/1) slip on exterior and interior. Hard, thin paste, very sparsely porous, fine, reddish yellow (7.5YR 6/6) fabric with occasional micaceous and tiny lime inclusions.

525. (No. 207): Handle fragment; Bath A, Room 1, found in 2006. pl. 23/525 and pl. 48/525.

Max. h $4.3 \mathrm{~cm}$., max. w $1.7 \mathrm{~cm}$., max. th 0.8 $\mathrm{cm}$.

Brown (7.5YR 5/4) slip on exterior and interior. Hard, thin paste, very sparsely porous, fine, light yellowish brown (10YR 6/4) fabric with no visible inclusions.

526. (No. 191): Handle fragment; Bath A, Room 4, found in 2006. pl. 23/526 and pl. 48/526.

Max. h $4.0 \mathrm{~cm}$., max. w $1.6 \mathrm{~cm}$., max. th 0.7 $\mathrm{cm}$.

Strong brown (7.5YR 5/6) and dark brown (7.5YR 3/2) mottled slip on exterior and interior. Average hardness; thin paste, non-porous, fine, light brown (7.5YR 6/4) fabric with frequent tiny lime inclusions.

A Lamp Fragment (pl. 48, no. 527)

A fragment of the middle part of lamp.

527. (No. 25): Body fragment; Domus, room 1, level 1, found in 2007. pl. 48/527.

Max. h 1.9 cm., max. w $2.8 \mathrm{~cm}$., max. th 0.4 $\mathrm{cm}$.

Dark brown (7.5YR 3/2) slip on exterior; pink (7.5YR 7/4) unslipped surface on interior. Average hardness; thin paste, very sparsely porous, fine, reddish yellow (7.5YR 6/6) fabric with frequent sand inclusions.

Body Fragments of Closed Forms (pl. 48, nos. 528-539)

Some with very intensive wheel-marks. 
528. (No. 568): Body fragment; surface find from the Village Boncuklar, found in 2005. pl. 48/528.

Max. h 1.5 cm., max. w 1.4 cm., max. th 0.3 $\mathrm{cm}$.

Dark brown (7.5YR 3/3) slip on exterior; greyish brown (10YR 3/2) unslipped surface on interior. Average hardness; thin paste, nonporous, fine, pale brown (10YR 6/3) fabric with no visible inclusions.

529. (No. 478): Body fragment; Bath A, room $2 \mathrm{~b}$, found in 2006. pl. 48/529.

Max. h $2.0 \mathrm{~cm}$., max. w $2.5 \mathrm{~cm}$., max. th 0.2 $\mathrm{cm}$.

Yellowish red (5YR 5/6) slip on exterior; pale brown $(2.5 \mathrm{Y} 7 / 3)$ and dark grey $(2.5 \mathrm{Y} 4 / 1)$ mottled unslipped surface on interior. Hard, thin paste, non-porous, fired to light brownish grey $(2.5 \mathrm{Y} 6 / 2)$ and reddish yellow (7.5YR $7 / 6$ ) fabric with no visible inclusions.

530. (No. 1267): Body fragment; Kimistene, Necropolis, surface find, found in 2005. pl. 48/530.

Max. h $1.5 \mathrm{~cm}$., max. w $2.4 \mathrm{~cm}$., max. th 0.3 $\mathrm{cm}$.

Reddish brown (5YR 5/4) slip on exterior, pink (7.5YR 7/4) unslipped surface on interior, Average hardness; thin paste, non-porous, fine, reddish yellow (7.5YR 7/6) fabric with no visible inclusions.

531. (No. 1326): Body fragment; Kimistene, summit of the Acropolis, found in 2005. pl. 48/531.

Max. h 2.4 cm., max. w 3.4 cm., max. th 0.4 $\mathrm{cm}$.

Brown (7.5YR 4/3) slip on exterior, pink (7.5YR 7/4) unslipped surface on interior, hard, thin paste, non-porous, fine, reddish yellow (7.5YR 7/6) fabric with no visible inclusions.
532. (No. 554): Body fragment; surface find from the Village Boncuklar, found in 2005. pl. 48/532.

Max. h $2.7 \mathrm{~cm}$., max. w $2.9 \mathrm{~cm}$., max. th 0.3 cm.

Brown (10YR 5/3) slip on exterior; very pale brown (10YR 7/3) unslipped surface on interior. Average hardness; thin paste, non-porous, fine, very pale brown (10YR 7/3) fabric with no visible inclusions.

533. (No. 55): Body fragment; Domus, room 6, level 1, found in 2007. pl. 48/533.

Max. h $3.2 \mathrm{~cm}$., max. w $2.7 \mathrm{~cm}$., max. th 0.3 $\mathrm{cm}$.

Dark greyish brown (10YR 4/2) slip on exterior; light yellowish brown $(2.5 \mathrm{Y} 6 / 3)$ and dark grey (2.5Y 4/1) mottled unslipped surface on interior. Hard, thin paste, non-porous, fired to light yellowish brown (2.5Y 6/3) and dark grey $(2.5 \mathrm{Y} 4 / 1)$ mottled fabric with no visible inclusions.

534. (No. 1455): Body fragment; Bath A, room 1, found in 2006. pl. 48/534.

Max. h $3.6 \mathrm{~cm}$., max. w $3.3 \mathrm{~cm}$., max. th $0.6 \mathrm{~cm}$. Reddish brown (2.5YR 4/4) shiny slip on exterior, light red (2.5YR 6/6) unslipped surface on interior, hard, thin paste, non-porous, fine, yellowish red (5YR 5/6) fabric with rare lime and sand inclusions.

535. (No. 218): Body fragment; Bath A, room 1, found in 2006. pl. 48/534.

Max. h 4.1 cm., max. w $2.6 \mathrm{~cm}$., max. th 0.5 $\mathrm{cm}$.

Dark brown (7.5YR 3/2) slip on exterior; pink (7.5YR 7/4) unslipped surface on interior. Hard, thin paste, non-porous, fine, light brown (7.5YR 6/4) fabric with no visible inclusions.

536. (No. 275): Body fragment; Bath A, Room 14, found in 2007. pl. 48/536. 
Max. h 4.3 cm., max. w $3.4 \mathrm{~cm}$., max. th 0.3 $\mathrm{cm}$.

Dark reddish brown (5YR 3/2) slip on exterior; red $(2.5 \mathrm{YR} 4 / 6)$ slip on upper exterior. Its below part has light red (2.5YR 6/6) unslipped surface on interior. Hard, thin paste, non-porous, fired to light red (2.5YR 6/6) and reddish yellow (5YR 6/6) mottled fabric with no visible inclusions.

537. (No. 338): Body fragment; Bath A, room 1, found in 2007. pl. 48/537.

Max. h 3.4 cm., max. w $4.5 \mathrm{~cm}$., max. th 0.5 $\mathrm{cm}$.

Brown (7.5YR 4/2) slip on exterior; reddish yellow (7.5YR 7/6) unslipped surface on interior. Average hardness; thin paste, very sparsely porous, fine, reddish yellow (7.5YR $6 / 6$ ) fabric with some tiny lime and rare micaceous inclusions.

538. (No. 1200): Body fragment; Kepez, surface find in the Cistern, found in 2005. pl. 48/538.

Max. h 3.7 cm., max. w 4.8 cm., max. th 0.6 $\mathrm{cm}$.

Brown (7.5YR 4/3) and reddish yellow (5YR 6/6) mottled slip on exterior; pink (7.5YR $7 / 3$ ) unslipped surface on interior. Hard, thin paste, very sparsely porous, fine, pink (7.5YR 7/4) fabric with infrequent calsite, sand and tiny lime inclusions.

539. (No. 1408): Body fragment; Kepez, surface find, found in 2005. pl. 48/539.

Max. h 7.8 cm., max. w 7.9 cm., max. th 0.8 $\mathrm{cm}$.

Dark brown (7.5YR 3/2) slip on exterior; pink (7.5YR 7/4) unslipped surface on interior. Hard, thin paste, non-porous, fine, reddish yellow (5YR 6/6) fabric with some small grit, sand and lime inclusions.
XII. HELLENISTIC COARSE WARE (pls. 23-25, nos. 541-580)

One of the largest groups of Hellenistic surface finds comprises the wheel-made cooking and plain ware that is classified as "Hellenistic coarse ware". Their typological and clay differences to Roman coarse ware, however, is not very distinctive. Most common forms have a large plain body and have no decoration. They should be locally manufactured.

Most common paste tones are reddish yellow (5YR 6/6-6/8-7/6, 7.5YR 6/6-7/6), yellowish red (5YR 5/6-5/8), red (2.5YR 5/6-5/8), light red (2.5YR 6/6-6/8), brown (7.5YR 5/4, 10YR 5/3), pale brown (10YR 6/3), grey (Gley $15 / \mathrm{N}, 10 \mathrm{YR}$ 6/1, 7.5YR 4/1, 5YR 5/1), reddish brown (5YR 5/4), pink (7.5YR 7/4) and light yellowish brown (10YR 6/4). Fine and hard fabric with grit, sand, lime, micaceous and grog in large sizes, visible even on the surface. Non-porous fabric with reddish yellow (5YR 6/6-7/6, 7.5YR 6/6-7/6), very pale brown (10YR 8/2-8/3-7/3), pink (7.5YR 8/38/4-7/4), light red (2.5YR 6/8), red (2.5YR 5/6), light reddish brown (5YR 6/4), grey $(2.5 \mathrm{Y} 6 / 1)$ and light yellowish brown (10YR 6/4) slips. Wall thickness differs between 4 and $10 \mathrm{~mm}$.

3 of 47 fragments belong to open and 44 to closed forms. 24 of them were found at Kepez, and the rest from Kimistene. There is no Hellenistic coarse ware from Hadrianopolis.

2nd-1st cent. B.C.

OPEN FORMS (pl. 23, no. 541) / Rim Fragments (pl. 23, no. 541)

It was not possible to assign any open sherds to any known forms. They belong to wide and large forms, probably for necessary household tasks such as mixing and preparing food; thus perhaps bowls. 
540. (No. 1117): Rim fragment; Kimistene, Acropolis, found in 2005. pl. 48/540.

Max. h $3.8 \mathrm{~cm}$., max. w $4.6 \mathrm{~cm}$., max. th 1.3 $\mathrm{cm}$.

Reddish yellow (5YR 6/6) slip on exterior and interior. Hard, non-porous, fine, reddish yellow (5YR 6/6) fabric with frequent large grit and sand inclusions.

541. (No. 828): Rim fragment; Kimistene, Cistern, eastern slope, found in 2005. pl. 23/541 and pl. 48/541.

Max. h 6.4 cm., d of rim $27.0 \mathrm{~cm}$., max. w $12.8 \mathrm{~cm}$. , max. th $1.1 \mathrm{~cm}$.

Reddish yellow (5YR 6/6) slip on exterior and interior. Hard, non-porous, fine, red $(2.5 \mathrm{YR}$ $5 / 8$ ) fabric with frequent tiny lime inclusions.

542. (No. 1067): Rim fragment; Kepez, found in 2005. pl. 49/542.

Max. h $5.5 \mathrm{~cm}$., d of rim $31.0 \mathrm{~cm}$., max. w 8.7 cm., max. th $1.5 \mathrm{~cm}$.

Light brown (7.5YR 6/4) unslipped surface on exterior and interior. Soft, sparsely porous fine, yellowish red (5YR 5/6) fabric with frequent small grit inclusions.

CLOSED FORMS (pls. 23-25, nos. 543580) / Rim Fragments (pls. 23-24, nos. 543-547)

They should be associated with storage or pouring activities in the household. Most of them are smaller sherds belonging to deep vessel forms with a globular or ovoid body, a constricted neck, a slightly thickened everted and outcurved rim and a flat or slightly rounded base. Thus, they could be cooking pots or jugs with a variation in size from from 9.4 to $24 \mathrm{~cm} \mathrm{~cm}$ in rim diameter.

543. (No. 1052): Rim fragment of a jug; Kepez, found in 2005. pl. 23/543 and pl. 49/543.

Max. h 3.1 cm., max. w 2.7 cm., max. th $0.7 \mathrm{~cm}$.
Very pale brown (10YR 8/2) slip on exterior and interior. Average hardness; non-porous, fine, reddish yellow (7.5YR 6/6) fabric with frequent tiny lime inclusions.

544. (No. 594): Rim fragment; Kimistene, Acropolis, southern slope, just below the summit, up to Deresemail creek, found in 2005. pl. 49/544.

Max. h $5.0 \mathrm{~cm}$., max. w $4.6 \mathrm{~cm}$., max. th 1.0 $\mathrm{cm}$.

Very pale brown (10YR 7/3) slip on exterior and interior rim. Reddish yellow (5YR 6/6) unslipped surface on lower interior. Hard, very sparsely porous, fine, reddish yellow (5YR 6/6) fabric with frequent tiny lime and some sand inclusions.

545. (No. 949): Rim fragment; Kepez, the surface find in the Cistern, found in 2005. pl. 23/545 and pl. 49/545.

Max. h 2.5 cm., d of rim 9.4 cm., max. w 3.5 cm., max. th $0.7 \mathrm{~cm}$.

Reddish yellow (5YR 6/6) slip on exterior and interior. Average hardness; non-porous, fine, light brown (7.5YR 6/6) fabric with some sand inclusions.

546. (No. 1156): Rim fragment; Kepez, the surface find in the Cistern, found in 2005. pl. 23/546 and pl. 49/546.

Max. h 2.0 cm., d of rim $13.6 \mathrm{~cm}$., max. w 4.8 cm., max. th $0.4 \mathrm{~cm}$.

Grey (2.5Y 6/1) slip on exterior; light grey (2.5Y 7/1) unslipped surface on interior. Hard, non-porous, fine, grey (10YR 6/1) fabric with some lime and large grit inclusions.

547. (No. 1273): Rim fragment of a casserole (?); Kimistene, Necropolis, found in 2005. pl. 24/547 and pl. 49/547.

Max. h 2.7 cm., d of rim $24.0 \mathrm{~cm}$., max. w 4.2 cm., max. th $1.3 \mathrm{~cm}$. 
Grey (2.5Y 6/1) unslipped surface on exterior; grey (Gley $16 / \mathrm{N})$ unslipped surface on interior. Hard, sparsely porous, fine, grey (Gley $15 / \mathrm{N}$ ) fabric with frequent lime and sand inclusions. Parallel: Sagona, Sagona 2004, 127, 441, fig. 118,4 (from Eski Köyeri Tepe 1; numbered as “BPS 30”).

\section{Base Fragments of Closed Forms (pl. 24,} nos. 548-556)

Ring bases of small jugs or table amphorae.

548. (No. 1108): Base fragment; Kepez, surface find in the Cistern, found in 2005. pl. 24/548 and pl. 49/548.

Max. h $1.4 \mathrm{~cm}$., d of base $8.0 \mathrm{~cm}$., max. w 6.5 $\mathrm{cm}$., max. th $1.0 \mathrm{~cm}$.

Reddish yellow (5YR 7/6) unslipped surface on exterior and interior. Average hardness; non-porous, fine, yellowish red (5YR 5/6) fabric with frequent tiny lime inclusions.

549. (No. 992): Base fragment; Kepez, found in 2005. pl. 24/549 and pl. 49/549.

Max. h $2.9 \mathrm{~cm}$., d of base $8.2 \mathrm{~cm}$., max. w 4.3 $\mathrm{cm}$, max. th $1.2 \mathrm{~cm}$.

Very pale brown (10YR 7/3) slip on exterior; light brownish grey (10YR 6/2) unslipped surface on interior. Average hardness; very sparsely porous, fired to dark grey (10YR 4/1) and pale brown (10YR 6/3) fabric with occasional tiny lime and sand inclusions.

550. (No. 1072): Base fragment; Kepez, found in 2005. pl. 24/550 and pl. 49/550.

Max. h $1.6 \mathrm{~cm}$., d of base $8.4 \mathrm{~cm}$., max. w 4.5 $\mathrm{cm}$., max. th $1.0 \mathrm{~cm}$.

Reddish yellow (7.5YR 7/6) unslipped surface all of surface. Average hardness; non-porous, fired to reddish yellow (5YR 6/6) fabric with infrequent tiny lime inclusions.
551. (No. 1228): Base fragment; Kepez, found in 2005. pl. 24/551 and pl. 49/551.

Max. h $2.7 \mathrm{~cm}$., d of base $10.0 \mathrm{~cm}$., max. w $8.8 \mathrm{~cm}$., max. th $1.2 \mathrm{~cm}$.

Reddish yellow (7.5YR 7/6) unslipped surface on exterior and interior. Pink (7.5YR 7/4) unslipped surface on interior. Average hardness; very sparsely porous, fine, reddish yellow (7.5YR 7/6) fabric with infrequent small grit inclusions.

552. (No. 1113): Base fragment; Kepez, surface find in the Cistern, found in 2005. pl. 24/552 and pl. 49/552.

Max. h $2.7 \mathrm{~cm}$., d of base $10.8 \mathrm{~cm}$., max. w $8.3 \mathrm{~cm}$., max. th $1.4 \mathrm{~cm}$.

Reddish yellow (7.5YR 6/6) slip on exterior. Exterior surface is burnished. Reddish yellow (5YR 6/6) slip on interior. Hard, very sparsely porous, fired to yellowish red (5YR 5/6) and grey (7.5YR 6/1) fabric with frequent tiny lime inclusions.

553. (No. 997): Base fragment; Kimistene, Acropolis, found in 2005. pl. 24/553 and pl. 49/553.

Max. h 4.9 cm., d of base 11.2 cm., max. w 8.8 $\mathrm{cm}$., max. th $1.2 \mathrm{~cm}$.

Light yellowish brown (10YR 6/4) unslipped surface on exterior; light brown (7.5YR 6/4) unslipped surface on interior. Soft, very sparsely porous, fired to brown (10YR 5/3) fabric with some sand inclusions.

554. (No. 1155): Base fragment; Kepez, surface find in the Cistern, found in 2005. pl. 24/554 and pl. 49/554.

Max. h $4.4 \mathrm{~cm}$., d of base $12.4 \mathrm{~cm}$., max. w $11.7 \mathrm{~cm}$. , max. th $1.4 \mathrm{~cm}$.

Light yellowish brown (10YR 6/4) thin slip on exterior; light brown (7.5YR 6/4) unslipped surface on interior. Hard, porous, fired to light yellowish brown (10YR 6/4) and 
brown (10YR 4/2) fabric with some tiny lime and sand inclusions.

555. (No. 1367): Base fragment; Kimistene, Acropolis, found in 2005. pl. 24/555 and pl. 50/555.

Max. h $3.5 \mathrm{~cm}$., d of base $12.8 \mathrm{~cm}$., max. w $6.9 \mathrm{~cm}$., max. th $1.1 \mathrm{~cm}$.

Reddish yellow (5YR 6/6) slip on exterior; reddish yellow (5YR 6/6) unslipped surface on interior. Average hardness; sparsely porous, fine, reddish yellow (5YR 6/8) fabric with frequent tiny lime inclusions.

556. (No. 1110): Base fragment; Kepez, surface find in the Cistern, found in 2005. pl. 24/556 and pl. 50/556.

Max. h $2.1 \mathrm{~cm}$., d of base $16.0 \mathrm{~cm}$., max. w $7.5 \mathrm{~cm}$., max. th $1.0 \mathrm{~cm}$.

Very pale brown (10YR 8/3) slip on exterior; brown (7.5YR 5/3) unslipped surface on interior. Average hardness; non-porous, fine, brown (7.5YR 5/4) fabric with some tiny lime and occasional micaceous inclusions.

Handle Fragment of Closed Form (pl. 50 , no. 557)

Vertical handle.

557. (No. 448): Handle fragment; Kimistene, Acropolis, southern slope, just below the summit, up to Deresemail creek, found in 2005. pl. 50/557.

Max. h 8.0 cm., max. w 3.9 cm., max. th 2.9 $\mathrm{cm}$.

Pink (7.5YR 8/3) slip on all of surface. hard, porous, fine, reddish yellow (5YR 6/6) fabric with some lime and occasional grog inclusions.

Body Fragment of Closed Form (pl. 25, nos. 579-580)
Some of them are decorated with simple painting or reliefing.

558. (No. 761): Body fragment; Kimistene, Acropolis, temple's terrace, illegally excavated area under the temple's podium, found in 2005. pl. 50/558.

Max. h $1.6 \mathrm{~cm}$., max. w 1.9 cm., max. th 0.6 $\mathrm{cm}$.

Reddish yellow (5YR 7/6) unslipped surface on exterior and interior. Hard, non-porous, fine, reddish yellow (5YR 6/6) fabric with frequent tiny lime and rare grog inclusions.

559. (No. 748): Body fragment; Kimistene, Acropolis, temple's terrace, illegally excavated area under the temple's podium, found in 2005. pl. 50/559.

Max. h 1.9 cm., max. w $2.2 \mathrm{~cm}$., max. th 0.7 $\mathrm{cm}$.

Pink (7.5YR 8/4) slip on exterior; light red (2.5YR 7/6) unslipped surface on interior. Hard, non-porous, fine, yellowish red (5YR 5/8) fabric with rare lime inclusions.

560. (No. 770): Body fragment; Kimistene, Acropolis, temple's terrace, illegally excavated area under the temple's podium, found in 2005. pl. 50/560.

Max. h 2.7 cm., max. w $2.2 \mathrm{~cm}$., max. th 0.6 $\mathrm{cm}$.

Reddish yellow (5YR 7/6) unslipped surface on exterior and interior. Hard, very sparsely porous, fine, reddish yellow (5YR 7/6) fabric with rare medium lime inclusions.

561. (No. 1055): Body fragment; Kepez, found in 2005. pl. 50/561.

Max. h 2.9 cm., max. w $3.2 \mathrm{~cm}$., max. th $0.6 \mathrm{~cm}$. Reddish yellow (5YR 6/6) unslipped surface on exterior and interior. Hard, non-porous, fine, red (2.5YR 5/6) fabric with some lime and rare medium grit inclusions. 
562. (No. 779): Body fragment; Kimistene, Acropolis, temple's terrace, illegally excavated area under the temple's podium, found in 2005. pl. 50/562.

Max. h $2.7 \mathrm{~cm}$., max. w $3.1 \mathrm{~cm}$., max. th 0.6 $\mathrm{cm}$.

Reddish yellow (5YR 6/6) unslipped surface on exterior and interior. Hard, non-porous, fine, reddish yellow (5YR 6/8) fabric with some lime, medium grit and rare micaceous inclusions.

563. (No. 785): Body fragment; Kimistene, Acropolis, temple's terrace, illegally excavated area under the temple's podium, found in 2005. pl. 50/563.

Max. h $3.3 \mathrm{~cm}$., max. w $3.3 \mathrm{~cm}$., max. th 0.7 $\mathrm{cm}$.

Reddish yellow (5YR 6/6) unslipped surface on exterior and interior. Hard, non-porous, fine, reddish yellow (5YR 6/8) fabric with some lime, small grit and rare micaceous inclusions.

564. (No. 729): Body fragment; Kimistene, Acropolis, temple's terrace, illegally excavated area under the temple's podium, found in 2005. pl. 50/564.

Max. h 2.5 cm., max. w $3.6 \mathrm{~cm}$., max. th 1.2 $\mathrm{cm}$.

Reddish yellow (5YR 6/6) unslipped surface on exterior and interior. Average hardness; non-porous, fine, yellowish red (5YR 5/6) fabric with some sand inclusions.

565. (No. 1177): Body fragment; Kepez, surface find in the Cistern, found in 2005. pl. 50/565.

Max. h $2.8 \mathrm{~cm}$., max. w $3.7 \mathrm{~cm}$., max. th 1.0 $\mathrm{cm}$.

Reddish yellow (7.5YR 7/6) slip on exterior; pink (7.5YR 7/4) unslipped surface on interior. Average hardness; non-porous, fine, red- dish yellow (5YR 6/8) fabric with infrequent tiny lime inclusions.

566. (No. 778): Body fragment; Kimistene, Acropolis, temple's terrace, illegally excavated area under the temple's podium, found in 2005. pl. 50/566.

Max. h $4.2 \mathrm{~cm}$., max. w $2.8 \mathrm{~cm}$., max. th 0.6 $\mathrm{cm}$.

Reddish yellow (5YR 6/6) unslipped surface on exterior and interior. Hard, non-porous, fine, reddish yellow (5YR 6/8) fabric with some lime, small grit and rare micaceous inclusions.

567. (No. 777): Body fragment; Kimistene, Acropolis, temple's terrace, illegally excavated area under the temple's podium, found in 2005. pl. 50/567.

Max. h $3.9 \mathrm{~cm}$., max. w $3.6 \mathrm{~cm}$., max. th 0.7 $\mathrm{cm}$.

Light red (2.5YR 6/8) slip on exterior; light red (2.5YR 7/6) unslipped surface on interior. Hard, thin paste, non-porous, fine, reddish yellow (5YR 6/6) fabric with frequent tiny lime inclusions.

568. (No. 1196): Body fragment; Kimistene, Acropolis, southern slope, just below the summit, up to Deresemail creek, found in 2005. pl. 50/568.

Max. h $4.6 \mathrm{~cm}$., max. w $4.0 \mathrm{~cm}$., max. th 0.9 $\mathrm{cm}$.

Pink (7.5YR 7/4) slip on exterior; reddish yellow (5YR 6/6) unslipped surface on interior. Exterior surface is burnished. Hard, non-porous, fired to reddish yellow (5YR 6/6) and grey $(7.5 \mathrm{YR} 5 / 1)$ fabric with occasional micaceous and rare lime inclusions.

569. (No. 1230): Body fragment; Kepez, found in 2005. pl. 50/569.

Max. h $5.0 \mathrm{~cm}$., max. w $4.7 \mathrm{~cm}$., max. th 1.0 $\mathrm{cm}$. 
Reddish yellow (7.5YR 7/6) slip on exterior; reddish yellow (5YR 6/6) unslipped surface on interior. Hard, non-porous, fired to yellowish red (5YR 5/6) fabric with frequent tiny lime inclusions.

570. (No. 1289): Body fragment; Kimistene, Acropolis, found in 2005. pl. 50/570.

Max. h $4.1 \mathrm{~cm}$., max. w $5.1 \mathrm{~cm}$., max. th 1.0 $\mathrm{cm}$.

Reddish yellow (2.5YR 6/6) unslipped surface on exterior and interior. Hard, non-porous, fine, yellowish red (5YR 5/6) fabric with infrequent tiny lime and sand inclusions.

571. (No. 1137): Body fragment; Kimistene, Acropolis, southern slope, just below the summit, up to Deresemail creek, found in 2005. pl. 50/571.

Max. h 4.9 cm., max. w $5.6 \mathrm{~cm}$., max. th 1.0 $\mathrm{cm}$.

Pink (7.5YR 7/4) unslipped surface on upper exterior; its below part has very pale brown (10YR 8/2) slip. Reddish yellow (2.5YR 6/6) unslipped surface on interior. A band in red $(2.5 \mathrm{YR} 5 / 6)$ on exterior. Exterior surface is burnished. Hard, non-porous, fine, red (2.5YR $5 / 6$ ) fabric with rare lime inclusions.

Parallel: Zolotarev 2005, 197, 207, fig. 9, 3 (a bulbous jug with flat or concave base, a short neck and a double-barrel handle and decorated with encircling, red-painted bands; 3rd century B.C.).

572. (No. 989): Body fragment; Kepez, found in 2005. pl. 50/572.

Max. h $5.1 \mathrm{~cm}$., max. w $5.3 \mathrm{~cm}$., max. th $0.4 \mathrm{~cm}$. Reddish yellow (5YR 7/6) slip on exterior; reddish yellow (5YR 6/6) unslipped surface on interior. Hard, very sparsely porous, fired to red (2.5YR 5/8) and pale brown (10YR 6/3) fabric with infrequent tiny lime, rare sand inclusions.
573. (No. 1229): Body fragment; Kepez, surface find in the Cistern, found in 2005. pl. 50/573.

Max. h 6.8 cm., max. w $4.2 \mathrm{~cm}$., max. th 1.2 $\mathrm{cm}$.

Reddish yellow (5YR 6/6) unslipped surface on exterior and interior. Hard, sparsely porous, fine, reddish yellow (5YR 6/6) fabric with some tiny lime, medium grit inclusions.

574. (No. 463): Body fragment; Kimistene, Acropolis, southern slope, just below the summit, up to Deresemail creek, found in 2005. pl. 50/574.

Max. h 3.0 cm., max. w 8.2 cm., max. th 0.9 $\mathrm{cm}$.

Red (2.5YR 5/6) slip on exterior; reddish yellow (5YR 6/4) unslipped surface on interior. A band in pinkish white (7.5YR 8/2) on exterior slip. Hard, very sparsely porous, fine, reddish yellow (7.5YR 6/6) fabric with some tiny lime inclusions.

575. (No. 1297): Body fragment; Kimistene, Acropolis, temple slope, illegally excavated pit, found in 2005. pl. 51/575.

Max. h $7.1 \mathrm{~cm}$., max. w $4.7 \mathrm{~cm}$., max. th 0.8 $\mathrm{cm}$.

Reddish yellow (5YR 6/6) slip on exterior; pink (5YR 7/4) unslipped surface on interior. Exterior surface is burnished. Hard, non-porous, fine, light reddish brown (5YR 6/4) fabric with frequent tiny lime, occasional sand inclusions.

576. (No. 863): Body fragment; Kimistene, Acropolis, temple slope, illegal excavation area, altar with boucranion, found in 2005 . pl. 51/576.

Max. h $7.3 \mathrm{~cm}$., max. w $5.8 \mathrm{~cm}$., max. th 0.8 $\mathrm{cm}$.

Pink (7.5YR 7/4) slip on exterior; very pale brown (10YR 7/4) unslipped surface on inte- 
rior. Exterior surface is burnished. Hard, nonporous, fired to very pale brown (10YR 7/4) and light red (2.5YR 6/8) fabric with frequent tiny lime and occasional sand inclusions.

577. (No. 1164): Body fragment; Kepez, surface find in the Cistern, found in 2005. pl. 51/577.

Max. h 6.6 cm., max. w 7.9 cm., max. th 0.9 $\mathrm{cm}$.

Reddish yellow (5YR 6/6) thin slip on exterior; reddish yellow (7.5YR 7/6) unslipped surface on interior. Exterior surface is burnished. Hard, non-porous, fired to yellowish red (5YR 5/6) and reddish yellow (7.5YR 6/6) fabric with some tiny lime and micaceous inclusions.

578. (No. 1379): Body fragment; Kepez, Necropolis, found in 2005. pl. 51/578.

Max. h $6.5 \mathrm{~cm}$., max. w $8.2 \mathrm{~cm}$., max. th 1.1 $\mathrm{cm}$.

Light brown (7.5YR 6/3) unslipped surface on exterior; light red (2.5YR 6/8) unslipped surface on interior. Traces of carbon on exterior. Hard, non-porous, light red (2.5YR 6/6) fabric with frequent tiny lime and occasional sand inclusions.

579. (No. 1151): Body fragment; Kepez, surface find in the Cistern, found in 2005. pl. 25/579 and pl. 51/579.

Max. h $6.7 \mathrm{~cm}$., max. w $9.5 \mathrm{~cm}$., max. th 1.0 $\mathrm{cm}$.

Very pale brown (10YR 8/2) slip on exterior; reddish yellow (7.5YR 6/6) unslipped surface on interior. Hard, very sparsely porous, fired to reddish yellow $(7.5 \mathrm{YR}$ 6/6) and brown (7.5YR 4/2) fabric with some tiny lime, medium grit and micaceous inclusions.

580. (No. 726): Body fragment; Kimistene, Acropolis, temple's terrace, illegally excavat- ed area under the temple's podium, found in 2005. pl. 25/580 and pl. 51/580.

Max. h 7.7 cm., max. w 8.5 cm., max. th 1.0 $\mathrm{cm}$.

Reddish yellow (5YR 7/6) slip on exterior; reddish yellow (5YR 6/6) unslipped surface on interior. Hard, non-porous, fine, reddish yellow (5YR 6/8) fabric with frequent tiny lime, medium grit and micaceous inclusions.

581. (No. 1293): Body fragment; Kepez, found in 2005. pl. 51/581.

Max. h 6.4 cm., max. w 9.9 cm., max. th 0.9 $\mathrm{cm}$.

Very pale brown (10YR 8/3) abrasion slip on exterior; light reddish brown (5YR 6/4) unslipped surface on interior. Hard, non-porous, fine, yellowish red (5YR 5/6) fabric with frequent tiny lime inclusions.

582. (No. 1162): Body fragment; Kepez, surface find in the Cistern, found in 2005. pl. 51/582.

Max. h 7.5 cm., max. w 10.1 cm., max. th 0.9 $\mathrm{cm}$.

Very pale brown (10YR 8/2) slip on exterior; reddish yellow (7.5YR 6/6) unslipped surface on interior. Average hardness; very sparsely porous, fine, reddish yellow (5YR 6/6) fabric with frequent tiny lime, medium grit and micaceous inclusions.

583. (No. 1154): Body fragment; Kepez, surface find in the Cistern, found in 2005. pl. $52 / 583$.

Max. h 11.2 cm., max. w 8.4 cm., max. th 0.6 $\mathrm{cm}$.

Very pale brown (10YR 8/2) slip on exterior; pink (7.5YR 7/4) unslipped surface on interior. Hard, porous, fine, pink (7.5YR 7/4) fabric with frequent tiny lime, rare micaceous and sand inclusions. 
584. (No. 697): Body fragment; Kimistene, Acropolis, temple's eastern slope, surface find, found in 2005. pl. 52/584.

Max. h 9.2 cm., max. w $9.2 \mathrm{~cm}$., max. th 0.3 $\mathrm{cm}$.

Reddish yellow (5YR 6/6) slip on exterior; pink (7.5YR 7/4) unslipped surface on interior. Hard, very sparsely porous, fired to yellowish red (5YR 5/6) and grey (5YR 5/1) fabric with frequent large grit and lime inclusions.

585. (No. 1474): Body fragment; Kepez, in the rock-cut grave, found in 2005. pl. 52/585. Max. h 4.6 cm., max. w 10.3 cm., max. th 1.4 $\mathrm{cm}$.

Light brown (7.5YR 6/4) unslipped surface on exterior and interior. Soft, sparsely porous, fine, brown (7.5YR 5/4) fabric with frequent tiny lime, sand and some medium grit inclusions.

586. (No. 1153): Body fragment; Kepez, surface find in the Cistern, found in 2005. pl. 52/586.

Max. h 9.4 cm., max. w 8.4 cm., max. th 1.5 $\mathrm{cm}$.

Very pale brown (10YR 8/3) slip on exterior; reddish yellow (5YR 7/6) unslipped surface on interior. Hard, non-porous, fine, light red (2.5YR 6/8) fabric with frequent tiny lime and some large grit inclusions in its interior surface.

\section{CONCLUSIONS}

In our surveys it was difficult to distinguish the characteristics of Middle and Late Iron Age sherds as well as their differences to each other. The lack of architectural evidence in the region is also making the characterization of Iron Age settlements in southwestern Paphlagonia difficult. Unfortunately very few sherds were found in any stratigraphic contexts; most of them are from the surface surveys. Kimistene is one of very few places in Paphlagonia where we have both locally manufactured Iron Age painted and grey ware with a limited typological repertory. It is, however, interesting to note that both wares do not occur in the same find spots: grey ware has a concentration in the stratified levels of the temple terrace, but painted ware was only found on the slopes of Kimistene's Acropolis. One of the very few stratified data for our ceramics comes from beneath the Roman temple's podium on Kimistene's Acropolis which has been dug out illegally at the beginning of the 2000s. Here we have at least three strata of c. $2 \mathrm{~m}$ deep, including mudbrick architecture and numerous grey ware sherds.

As the similarity of the ceramic evidence of southwestern Paphlagonia with that of Boğazköy, Gordion, Oluz Höyük, İkiztepe, Alişar IV, Kaman-Kalehöyük and Çadır Höyük shows, in the Iron Age southwestern Paphlagonia definitely had contact with the ceramic traditions of the Central Anatolian Plateau and the Central Black Sea area. The Late Iron Age is often associated with the Achaemenid presence in Paphlagonia (Matthews 2009, 155-156; and for Achaemenid presence in Paphlagonia: Johnson 2010). In our surveys we have almost no indication for the physical existence of the Achaemenids in our survey area, as the Achaemenid rule is hard to discern through its material culture. In both the Iron Age and the Hellenistic period imported pottery is very rare; most of the products should be locally manufactured. Our Iron Age and Hellenistic surface material 
shows a continuity of form and decoration from the preceding Iron Age into the Hellenistic era, as also attested on the Konya plain and in the Amasya region (Matthews 2009, 173; Dönmez 2005a, 69).

As Matthews indicates (Matthews 2009, 173), Alexander's march and the progress of the Hellenistic age across Anatolia had no significant material impact upon the inhabitants of Inner Paphlagonia. Beside that, our knowledge about Hellenistic Paphlagonia is extremly limited: Strabo wrote that before his time Paphlagonia was ruled by several kings (12.3.41). In alliance with the Bithynian king, Nicomedes III Euergetes, Mithradates VI conquered Paphlagonia in 108/7 B.C. It seems that during the Hellenistic period the Kimistene region had almost no contact with the coastal Greek cities, although the first Greek influences should had began in this period. Thus, we should expect a closed and isolated administration in southwestern Paphlagonia during this period.

Most of our Hellenistic pottery results depend on Kimistene. Hellenistic material from this hill-top site proves that this site was settled beginning at least in the Iron Age and continued through the entire Hellenistic period without a break. It should have had a specific (religious?) function during the Hellenistic period; perhaps the Roman temple in Kimistene had an Iron Age and Hellenistic predeccessor in wooden form. Since Hellenistic settlements in the area are rare, the material from Kimistene is immensely important. Kepez should also have had a specific function as a Hellenistic grave site, but the use of this site continued until the Early Byzantine period.

In the Hellenistic period locally manufactured pottery in southwestern Paphlagonia was very conservative in terms of typology and decoration and this did not change for a long time. Their clay characteristics with either red or brown fabric are very homogeneous. They mostly consist of basic open forms, such as dining vessels (incurved rim bowls, fish plates, everted rim bowls, dishes etc.), imitated from western and northern workshops, sometimes with banded decoration. As in the Iron Age, Hellenistic finds from southwestern Paphlagonia are also connected with Central and Northern Anatolian Hellenistic pottery traditions. Hellenistic sherds from Kimistene are similar to the finds from Oluz Höyük in Amasya. The pottery from Kimistene and the rest of southwestern Paphlagonia, especially the painted examples, indicates religious-ritual activities. Thus, they do not really reflect daily Hellenistic life in this part of the ancient world.

\section{NOTES AND \\ ACKNOWLEDGEMENTS}

All the plates were drawn by G. Kan Şahin who also took the photographs with C. Şahin (Çanakkale); the maps were produced by $\mathrm{S}$. Patac1 (Ardahan). In the catalogue section numbers in brackets indicate find numbers of the field project. Abbreviations in the catalogue: th: thickness; h: height; w: width; d: diameter; and max.: maximum. For this article The Munsell Soil Color Charts (2009) has been used. These sherds are being stored today in the Museum of Amasra as well as at the excavation container of the site.

We would like to thank to following colleagues for their help with various aspects of this paper (alphabetically): Olivera Ilić (Belgrade), Chris Lightfoot (New York), John Lund (Copenhagen), Sami Pataci (Ardahan) and Milica Tapavički-Ilić (Belgrade). 
Laflı, Kan Şahin - Iron Age and Hellenistic Ceramics...(9-166) Archaeology and Science 7 (2011)

\section{BIBLIOGRAPHY}

\section{Akurgal, E. Budde, L 1956}

Vorläufiger Bericht über die Ausgrabungen in Sinope, Ankara.

\begin{abstract}
Alkım, U.B., Alkım, H. and Bilgi, Ö. 2003
İkiztepe II Üçüncü, Dördüncü, Beşinci, Altıncı, Yedinci Dönem Kazılart: 1976-1980, Türk Tarih Kurumu Yayınları, V. Dizi, Sayı 39, Ankara.
\end{abstract}

Bilde, P. G. 2010

Mouldmade Bowls, In: N. A. Lejpunskaja, P. G. Bilde, J. M. Højte, V. V. Krapivina and S. D. Kryžickij (eds.), The Lower City of Olbia (Sector $N G S$ ) in the 6th Century BC to the 4th Century $A D$, Vols. 1-2, Aarhus University Press: 269-288, pl. 168-195.

\section{Bilgi, Ö. 1999}

Samsun-İkiztepe Arkeolojik Kazıları Tepe III Çalışmaları. 1993 ve 1994 Dönemi Sonuçları, Anadolu Araştırmalarl/Jahrbuch für Kleinasiatische Forschung XV: 137-165.

\section{Chalier, I. 2008}

Le cyclope d'Euripide sur un bol à reliefs de Porsuk, Revue Archéologique 2008/1-no 45, Presses Universitaires de France: 93-105.

\section{Doonan, O. 2004}

Sinop Landscapes, Exploration Connection in a Black Sea Hinterland, Pennsylvania.

\section{Dönmez, Ş. 2005a}

Amasya province in the Iron Age, in: A. Çilingiroğlu, G. Darbyshire (eds.), Anatolian Iron Ages 5, British Institute at Ankara Monograph 31 (London 2005) 65-74.

\section{Dönmez, Ş. 2005b}

Orta Karadeniz Bölgesi'nde Önemli Bir Demir Çağı Yerleşmesi: Ladik-Köyiçi Tepesi/İkizarı,
Anadolu Araştırmaları/Jahrbuch für Kleinasiatische Forschung XVIII/1: 65-109.

\section{Dönmez, Ş. 2010a}

Oluz Höyük Kazısı Üçüncü Dönem (2009) Çalışmaları: Değerlendirmeler ve Sonuçlar, CollAn IX: 275-306.

\section{Dönmez, Ş. 2010b}

Kašku Ülkesi'nin Önemli Kenti Amasya-Oluz Höyük 2007 ve 2008 Dönemi Çalışmaları Genel Değerlendirmeler ve Ön Sonuçlar, T.C. Amasya Valiliği Yayın No: 48 (Ankara 2010).

\section{Dönmez, Ş. 2011}

Oluz Höyük Kazısı Dördüncü Dönem (2010) Çalışmaları: Değerlendirmeler ve Sonuçlar, CollAn X: 103-128.

\section{Fünfschilling, S., Laflı, E. 2012}

Hadrianopolis II: Glasfunde des 6. und 7. Jhs. aus Hadrianupolis, Paphlagonien [Türkei], Internationale Archäologie 123 (Rahden/Westf. 2012).

\section{Genz, H. 2004}

Büyükkaya I, Die Keramik der Eisenzeit, Funde aus den Grabungskampagnen 1993 bis 1998, Boğazköy-Hattuša 21, (Mainz am Rhein 2004).

\section{Gorny, R.L. 2009}

Çadır Höyük, The 2008 Season, Oriental Institute 2008-2009 Annual Report, 18-35, $<$ http://oi.uchicago.edu/pdf/ar/01-10/08-09/0809_AnnualReport.pdf> (02/10/2012).

\section{Henrickson, R. C. 2005}

The Local Potter's Craft at Phrygian Gordion, in: L. Kealhofer, (eds.), The Archaeology of Midas and the Phrygians, Recent Work at Gordion, (Philadelphia 2005) PA, 124-135.

\section{Johnson, P. 2010}

Landscapes of Achaemenid Paphlagonia, Publicly 
Accessible Penn Dissertations, Paper 273 (Diss.; University of Pennsylvania 2010) <http://repository.upenn.edu/edissertations/273> (11/01/2012).

\section{Kealhofer et al. 2010}

Patterns of Iron Age Interaction in Central Anatolia: Three Sites in Yozgat Province, Anatolian Studies 60, 71-92.

\section{Lafli, E. 2006}

Hellenistic and Roman Ceramic Archaeology on the Southern Black Sea Coast: An Overview of the Turkish Archaeological Literature related to Ceramic Archaeology of Paphlagonia and Pontus, in: B. D. Erciyas, E. Kopraman (eds.), Black Sea Studies Symposium Proceedings 2004 Ankara, (Istanbul 2006) 189-202.

\section{Laflı, E., Christof, E. 2011}

Der kaiserzeitliche Tempel von Asartepe/Kimistene in der Chora des paphlagonischen Hadrianopolis - Ergebnisse der Prospektion von 2005, Istanbuler Mitteilungen 61: 233-285.

Laflı, E., Christof, E. 2012a

Hadrianopolis I: Inschriften aus Paphlagonia, BAR, Int. Ser. 2366 (Oxford 2012).

\section{Laflı, E., Christof, E. 2012b}

Drei neu entdeckte Phallossteine aus der Chora von Hadrianopolis, in: G. R. Tsetskhladze (ed.), The Black Sea, Paphlagonia, Pontus and Phrygia in Antiquity. Aspects of Archaeology and Ancient History, BAR, Int. Ser. 2432 (Oxford 2012) 163170.

Laflı, E., Kan Şahin, G. 2012a

Terra Sigillata and Red-Slipped Ware from Hadrianopolis in Southwestern Paphlagonia, Anatolica Antiqua 20: 45-120.

\section{Lafl, E., Kan Şahin, G. 2012b}

Pottery from southwestern Paphlagonia I: Terra Sigillata and Red-Slipped Ware, in: B. Ramminger, O. Stilborg (eds.), Naturwissenschaftliche Analysen vor-und frühgeschichtlicher Keramik II. Dritter und vierter internationaler Workshop für junge Wissenschaftlerinnen und Wissenschaftler in Hamburg am 9. Februar 2010 und 7. Februar 2011. Universitätsforschungen zur prähistorischen Archäologie aus der Abteilung Vor- und Frühgeschichtliche Archäologie der Universität Hamburg (Bonn 2012) 171-208.

\section{Laflı, E., Kan Şahin, G. 2013 in print}

A Preliminary Report on the Iron Age and Hellenistic Ceramics from Southwestern Paphlago-

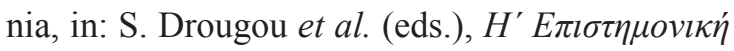

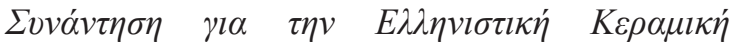
(Iwóvviva 2009) / H'Scientific Meeting on Hellenistic Pottery, Ioannina 2009. Proceedings (Athens 2013, in print).

\section{Lawall, M. 2005}

Negotiating Chronologies: Aegean Amphora Research, Thasian Chronology, and Pnyx III, in: V. F. Stolba, L. Hannestad (eds.), BSS 3, Chronologies of the Black Sea Area in the Period c. 400100 BC, Arrhus: 31-67.

\section{Magie D. 1950}

Roman Rule in Asia Minor to the End of the Third Century after Christ, Princeton, NJ.

\section{Matthews, R. 2009}

A Dark Age, Grey Ware and Elusive Empirs: Paphlagonia through the Iron Age, 1200-330 BC, in: R. Matthews, C. Glatz (eds.), At Empire's Edge: Project Paphlagonia. Regional Survey in North-Central Turkey. British Institute in Ankara, BIAA Monograph 44: 149-171. 
Matthews, R., Metcalfe, M. and Cottica, D. 2009

Landscapes with Figures: Paphlagonia through the Hellenistic, Roman and Byzantine Periods, 330 BC-AD 1453, in: R. Matthews, C. Glatz (eds.), At Empire's Edge: Project Paphlagonia. Regional Survey in North-Central Turkey. British Institute in Ankara, BIAA Monograph 44: 173199 and 216-226.

\section{Mitchell, S. 1993}

Anatolia: Land, Men, and Gods in Asia Minor, Vol. 1: The Celts in Anatolia and the Impact of Roman Rule, Oxford.

\section{Polat, G. 1993}

A Group of Phrygian Pottery from the Archaeological Museum of İstanbul.

\section{Rotroff, S. I. 1997}

Hellenistic Pottery, Athenian and Imported Wheelmade Table Ware and Related Material. The Athenian Agora XXIX, Part 1-2, Princeton, NJ.

\section{Omura, S. 1996}

1994 Yılı İç Anadolu'da Yürütülen Yüzey Araştırmaları, in: T. C. Kültür ve Turizm Bakanlığı, Kültür Varlıkları ve Müzeler Genel Müdürlüğü, 13. Araştırma Sonuçları Toplantısı, Cilt 2, (Kültür ve Turizm Bakanlığ1 Yayınları/ Kültür Varlıkları ve Müzeler Genel Müdürlüğü Yayınları), Ankara: 243-272.

\section{Omura, S. 2006}

Preliminary Report of the 2005 General Survey in Central Anatolia, Kaman-Kalehöyük 15, Anatolian Archaeological Studies Vol. XV, Ibaraki: 63-102.

\section{Omura, S. 2007}

Preliminary Report of the General Survey in Central Anatolia (2006), Kaman-Kalehöyük 16, Anatolian Archaeological Studies Vol. XVI, Ibaraki: 45-84.

\section{Omura, S. 2008}

Preliminary Report of the General Survey (2007), Kaman-Kalehöyük 17, Anatolian Archaeological Studies Vol. XVII, Ibaraki: 63-102.

\section{Özdoğan, A., Marro, C. and Tibet, A. 1997} 1995 Y1lı Kastamonu Yüzey Araştırması, in: T. C. Kültür ve Turizm Bakanlığı, Kültür Varlıkları ve Müzeler Genel Müdürlüğü, 14. Araştırma Sonuçları Toplantısı, Cilt 2, (Kültür ve Turizm Bakanlığı Yayınları/Kültür Varlıkları ve Müzeler Genel Müdürlüğü Yayınları), Ankara: 303-330.

Özdoğan, A., Marro, C. and Tibet, A. 1999 Kastamonu Yüzey Araştırması 1997 Y1lı Çalışmaları, in: T. C. Kültür ve Turizm Bakanlığı, Kültür Varlıkları ve Müzeler Genel Müdürlüğü, 16. Araştırma Sonuçları Toplantısı, Cilt 2, (Kültür ve Turizm Bakanlığı Yayınları/Kültür Varlıkları ve Müzeler Genel Müdürlüğü Yayınları), Ankara: 219-244.

\section{Özsait, M., Özsait, N. 1996}

Şeref Höyük: Komama, Anadolu Araştırmalarl/ Jahrbuch für Kleinasiatische Forschung XIV, İstanbul: 361-389.

\section{Sagona, A., Sagona, C. 2004}

Archaeology at the North-East Anatolian Frontier, I: An Historical Geography and Field Survey of the Bayburt Provience, Ancient Near Eastern Studies, Supplement Series 14, Louvain, Peeters Press.

\section{Summerer, L. 2007}

Greeks and Natives on the southern Black Sea Coast in Antiquity, in: G. Erkut, and S. Mitchell (eds.), The Black Sea: Past, Present and Future, Proceedings of the International, Interdisciplinary Conferance, Istanbul, 14-16 October 2004, British Institute at Ankara Monograph 42, British Institute at Ankara, London: 27-36. 
Stewart, S.M. 2010

Gordion after the Knot: Hellenistic Pottery and

Culture (Unpubl. Diss.; University of Cincinnati).

Şenyurt, S. Y., Kamış, Y. and Akçay, A. 2005

Demir Çağı Çanak Çömlek Buluntuları, in: S.Y.

Şenyurt, (ed.), Tasmasor Erzurum Ovası'nda Bir

Demir Çă̆ Yerleşmesi, Bakü-Tiflis-Ceyhan Ham

Petrol Boru Hattı Projesi Arkeolojik Kurtarma

Kazıları Yayınları: 4, Ankara, 85-177. <http:// agt.si.edu/_images/uncover_more/site_reports/_ site_report_pdf/turkey/Tasmasor_Turkish.pdf $>$ $(02 / 10 / 2012)$.

\section{Şenyurt, S.Y., Ekmen, H. 2005}

Tetikom Pasinler Ovası'nda Bir Demir Çă̆ı Yerleşmesi, Bakü-Tifllis-Ceyhan Ham Petrol Boru Hattı Projesi Arkeolojik Kurtarma Kazıları Proje Dokümanları: 1, Ankara.

$<$ http://agt.si.edu/_images/uncover_more/site_reports/_site_report_pdf/turkey/Tetikom_Turkish. pdf $>(02 / 10 / 2012)$.

Toteva, G. D. 2007

Local Cultures of Late Achaemenid Anatolia (Unpubl. Diss.; University of Minnesota 2007).

Voigt, M.M., et al. 1997

Fieldwork at Gordion: 1993-1995, Anatolica 23: $1-59$.

\section{Zolotarev, M.I. 2005}

A Hellenistic Ceramic Deposit from the Northeastern Sector of Chersonesos, in: V.F. Stolba and L. Hannestad (eds.), Chronologies of the Black Sea Area in the Period c. 400-100 BC, 193-216, Aarhus: Aarhus University Press.

\section{Zoroğlu, L. 1979}

Eskiyapar'da Bulunan Kızılırmak Havzası (Galat Denilen) Boyalı Seramikleri, VIII. Türk Tarih Kongresi, I.Cilt, Ankara: 239-252.

\section{REZIME \\ GVOZDENO DOBA I HELENISTIČKA KERAMIKA IZ JUGOZAPADNE PAFLAGONIJE}

\section{KLJUČNE REČI: PAFLAGONIJA, KIMISTENA, HADRIJANOPOLIS, CRNO MORE, MALA AZI- JA, TURSKA, GVOZDENO DOBA, FRIGIJSKI, HELENISTIČKI.}

Paflagonija je stara oblast na crnomorskoj obali središnje severne Anadolije, koja se na zapadu graničila sa Bitinijom, na istoku sa Pontskom kraljevinom, a na jugu sa Galatijom. Arheološka ekipa sa univerziteta Dokuz Eylül iz Izmira je između 2005. i 2008. izvršila rekongosciranje, kao i iskopavanje u jugozapadnom delu Paflagonije. U ovom obimnom radu, dat je detaljan prikaz keramičkih nalaza i perioda gvozdenog doba i Helenizma, a koji su otkriveni tokom napred navedenih istraživanja. Prikazana je tipologija različitih keramičkih fragmenata, uglavnom sakupljenih sa površine. Za razliku od ostalih lokaliteta iz perioda kojima se ovde bavimo, jasnije informacije potiču sa Kimistene, lokaliteta na visoravni smeštenoj unutar istraživane oblasti u jugozapadnoj Paflagoniji. Ova studija je dovela do razdvajanja dveju keramičkih grupa: iz perioda gvozdenog doba i iz Helenizma, koja usled toga predstavlja prvu detaljnu tipologiju helenističke keramike iz turskog crnomorskog priobalja. 

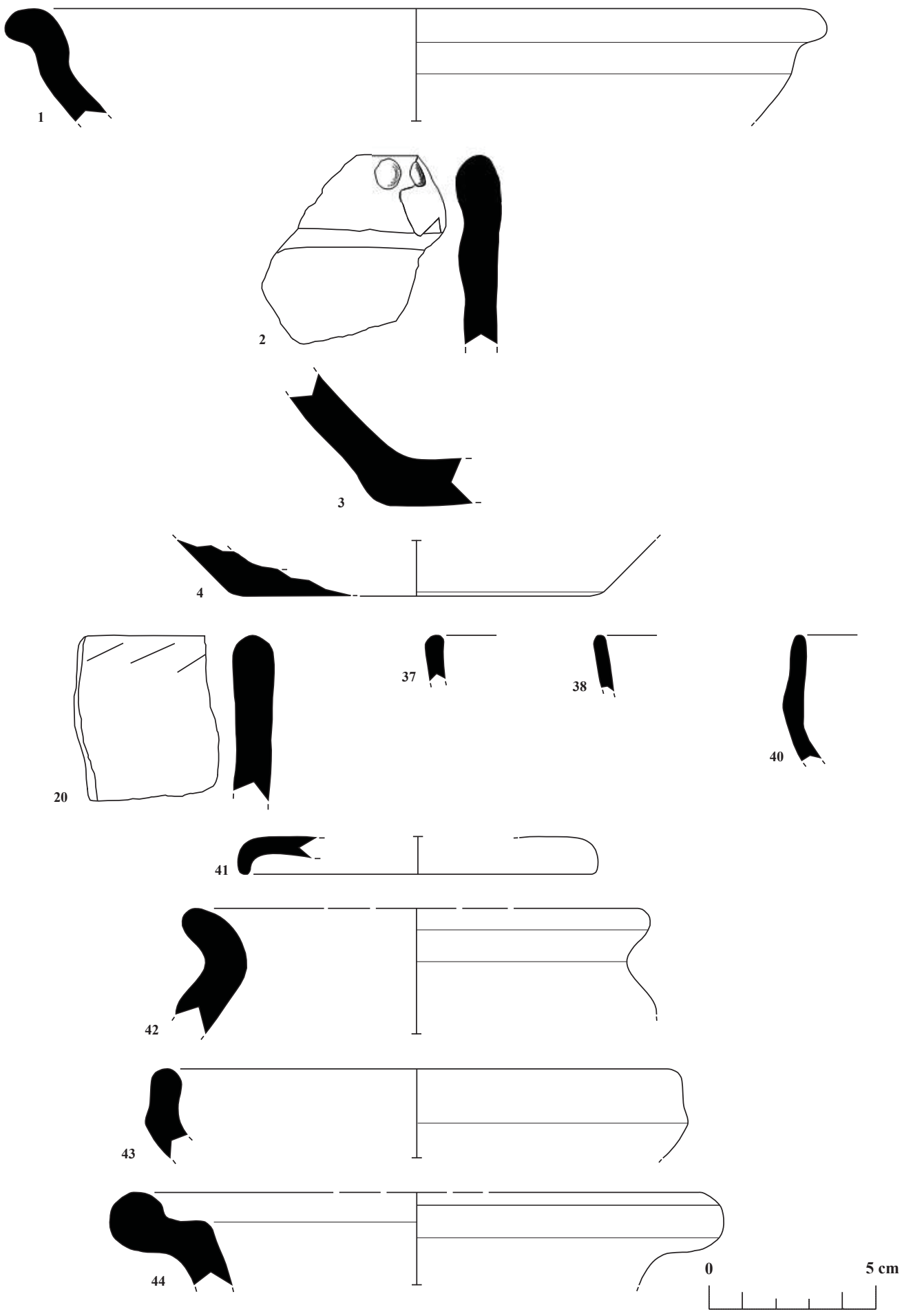

PI. 1: Nos. 1-20: Pre-Iron Age pottery; nos. 1-2: Rim fragments; nos. 3-4: Base fragments of open forms; no. 20: A rim fragment of a closed form; and nos. 37-44: Iron Age grey ware, rim fragments of open forms. 

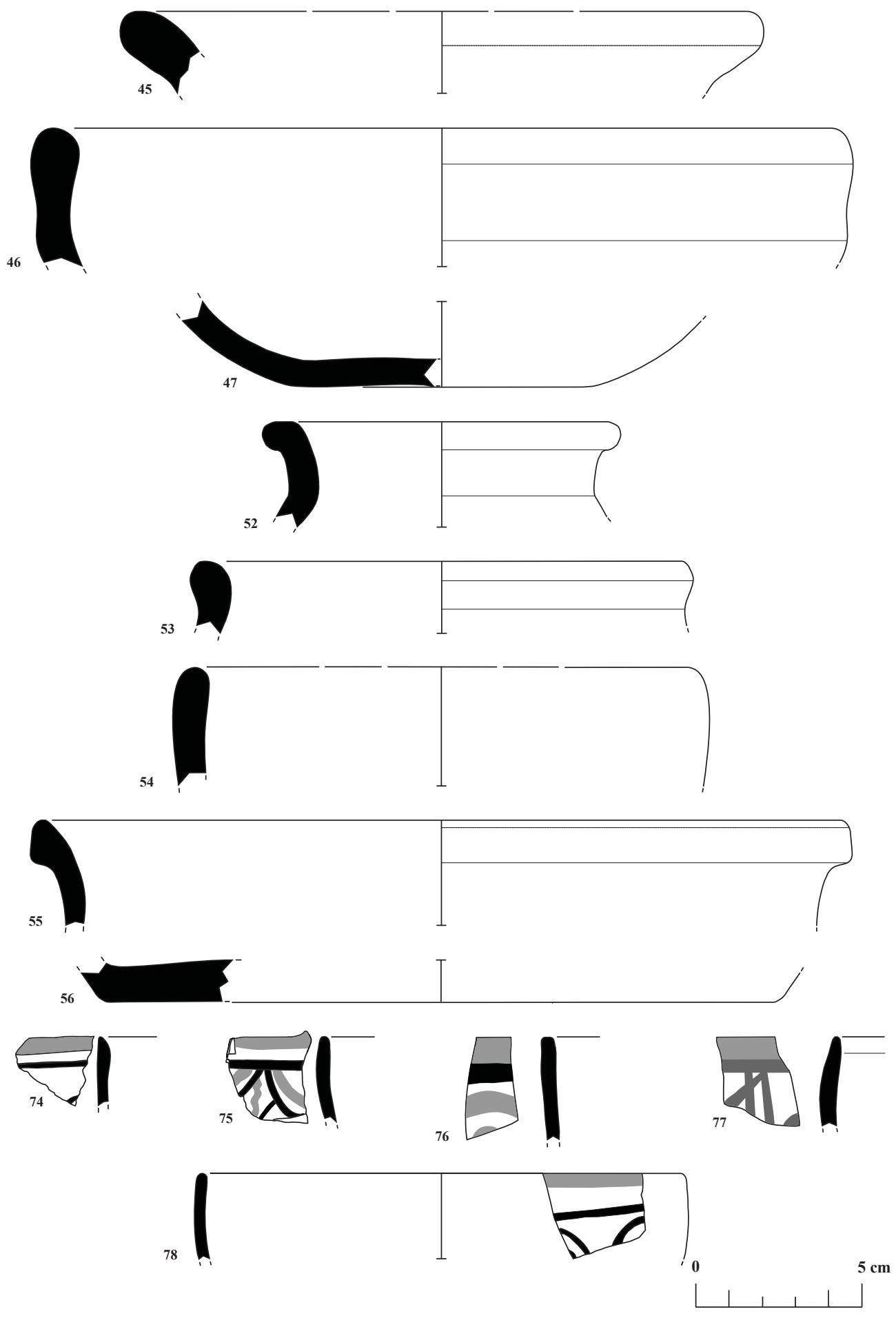

PI. 2: Nos. 45-56: Iron Age grey ware; nos. 45-46: Rim fragments of open forms; no. 47: A base fragment of open forms; nos. 52-55: Rim fragments of closed forms; no. 56: A base fragment of a closed form; and nos. 74-78: Iron Age painted ware, rim fragments of a bowl form. 

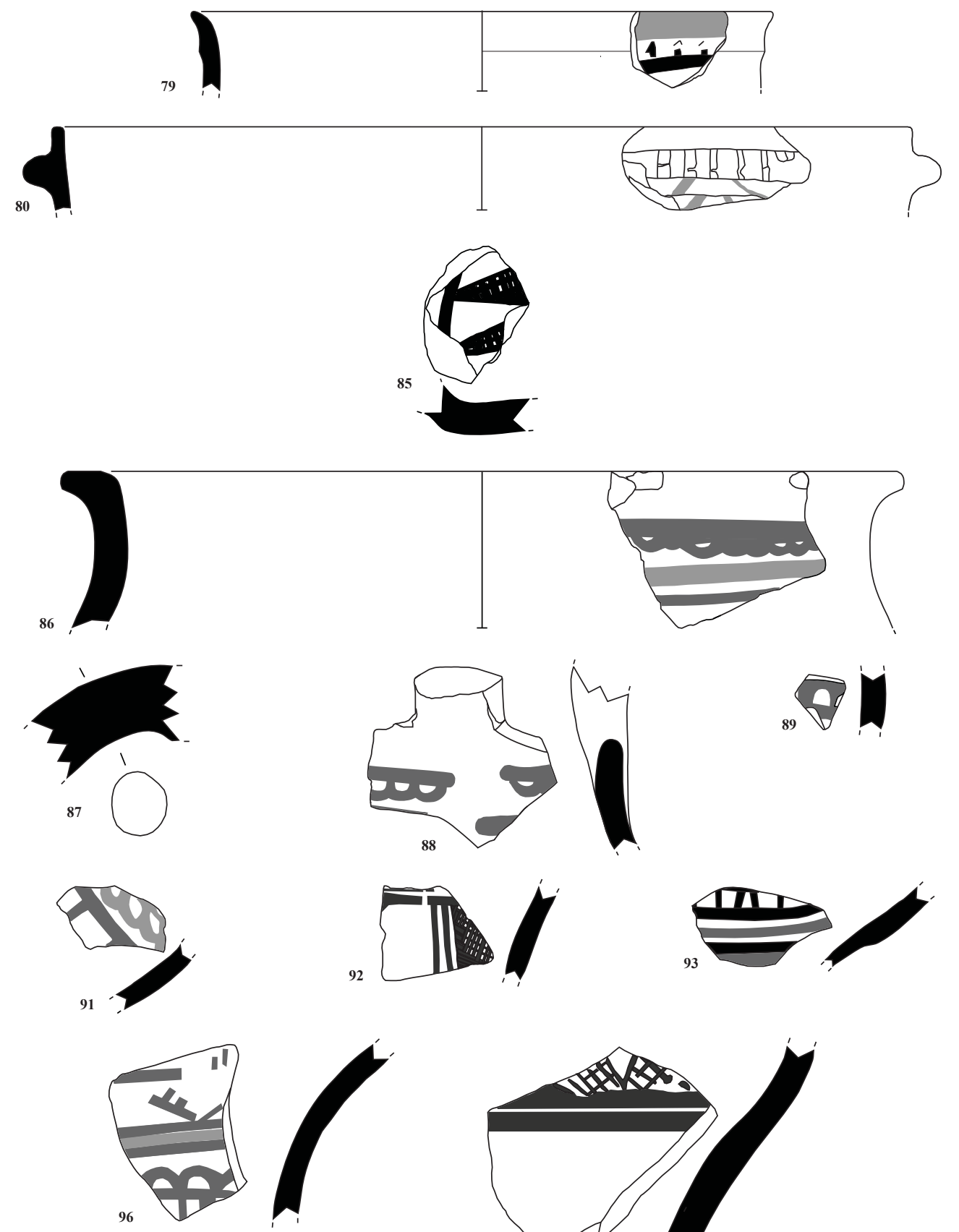

88
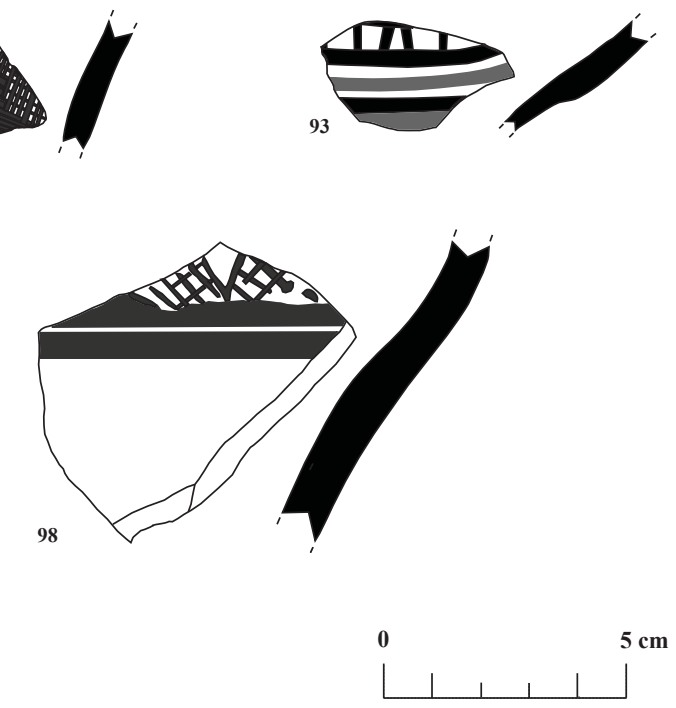

Pl. 3: Nos. 79-98: Iron Age painted ware; nos. 79-80: rim fragments of a bowl form; no. 85: A body fragment of open forms; no. 86: A rim fragment of closed forms; nos. 87-88: Handle fragments of closed forms; and nos. 89-98: Body fragments of closed forms. 


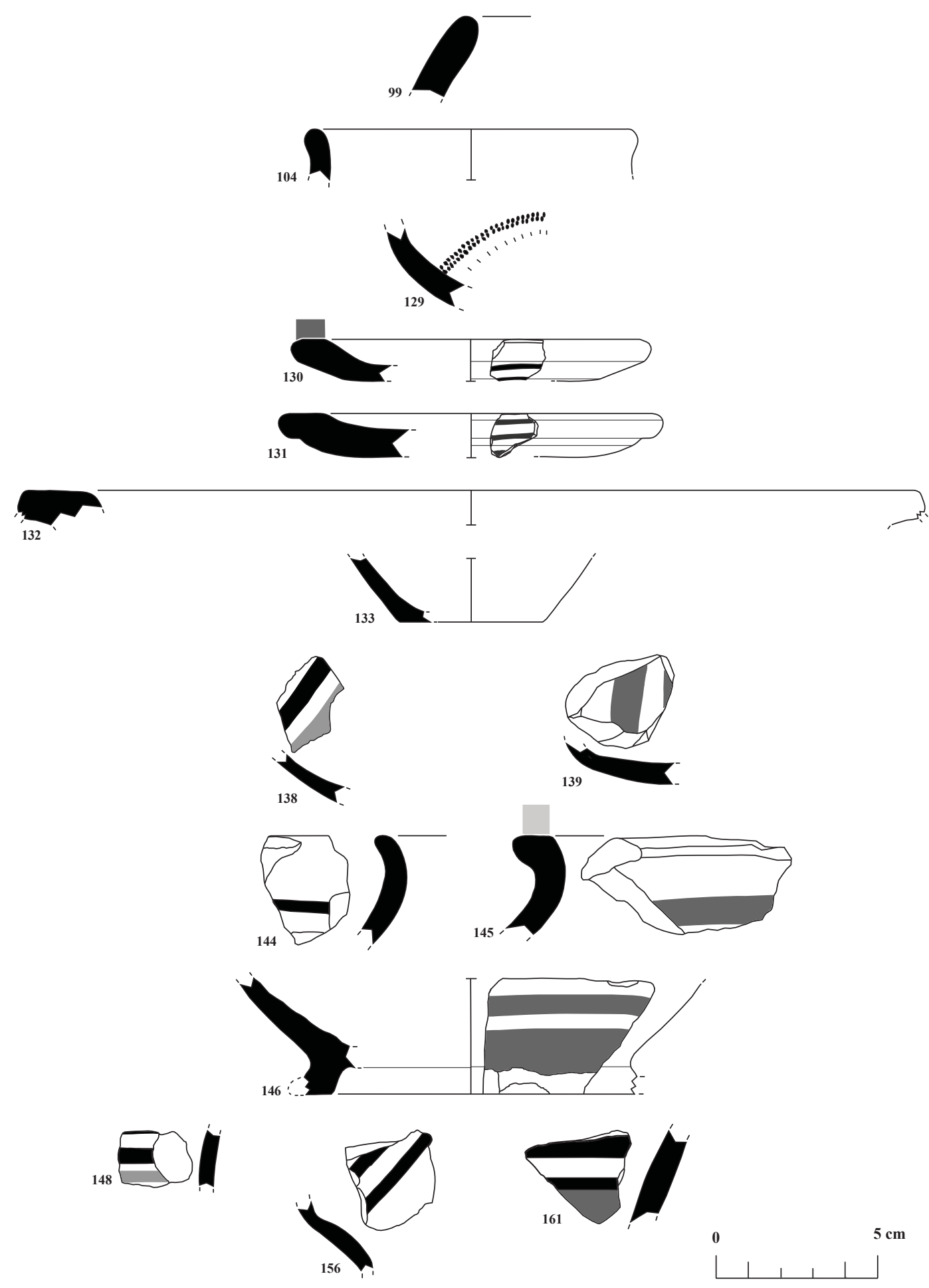

Pl. 4: Nos. 99-104: Iron Age coarse ware; no. 99: A rim fragment of open forms; nos. 100103: Body fragments of open forms; no. 104: Rim fragment of closed forms; nos. 106-128: Body fragments of closed forms; no. 129: A Pontic skyphos fragment; nos. 130-161: Hellenistic painted ware; nos. 130-132: Rim fragments of open forms; no. 133: A base fragment of open forms; nos. 138139: Body fragments of open forms; nos. 144-145: Rim fragments of closed forms; no. 146: A base fragment of closed forms; and nos. 148-161: Body fragments of closed forms. 

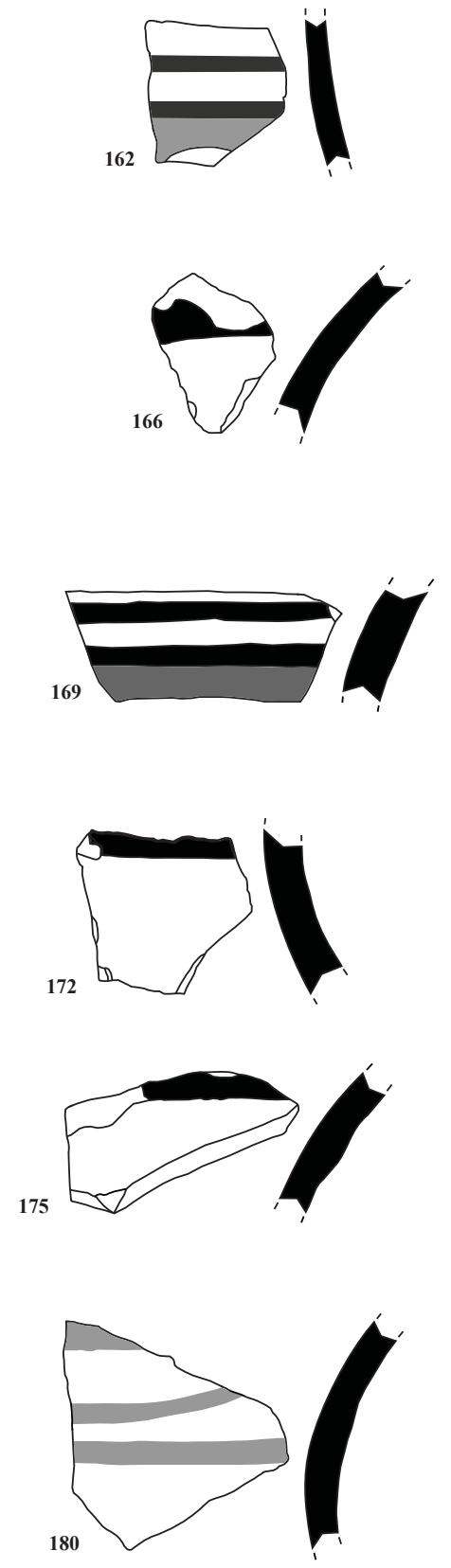
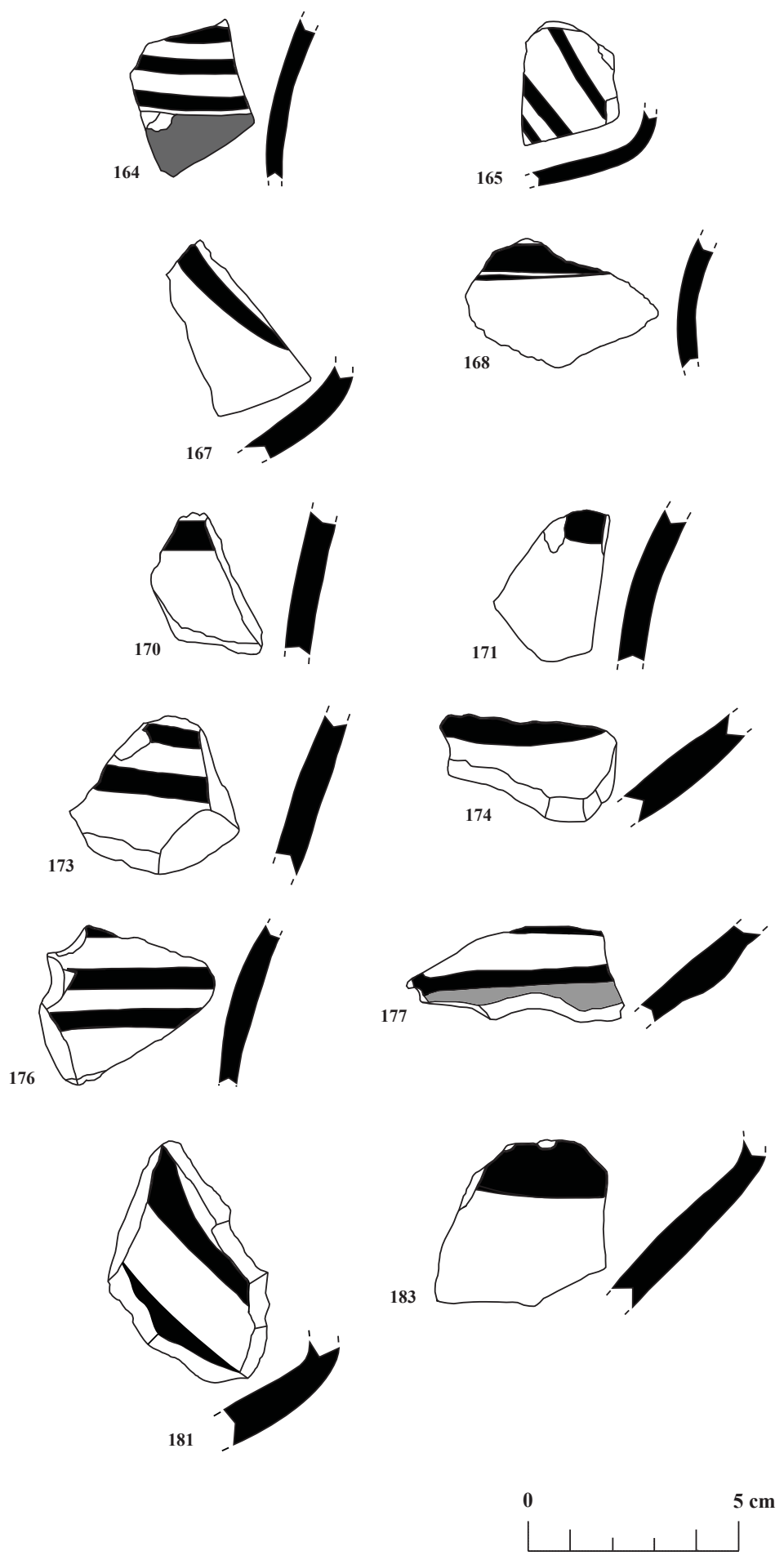

Pl. 5: Nos. 162-183: Hellenistic painted ware, body fragments of closed forms. 

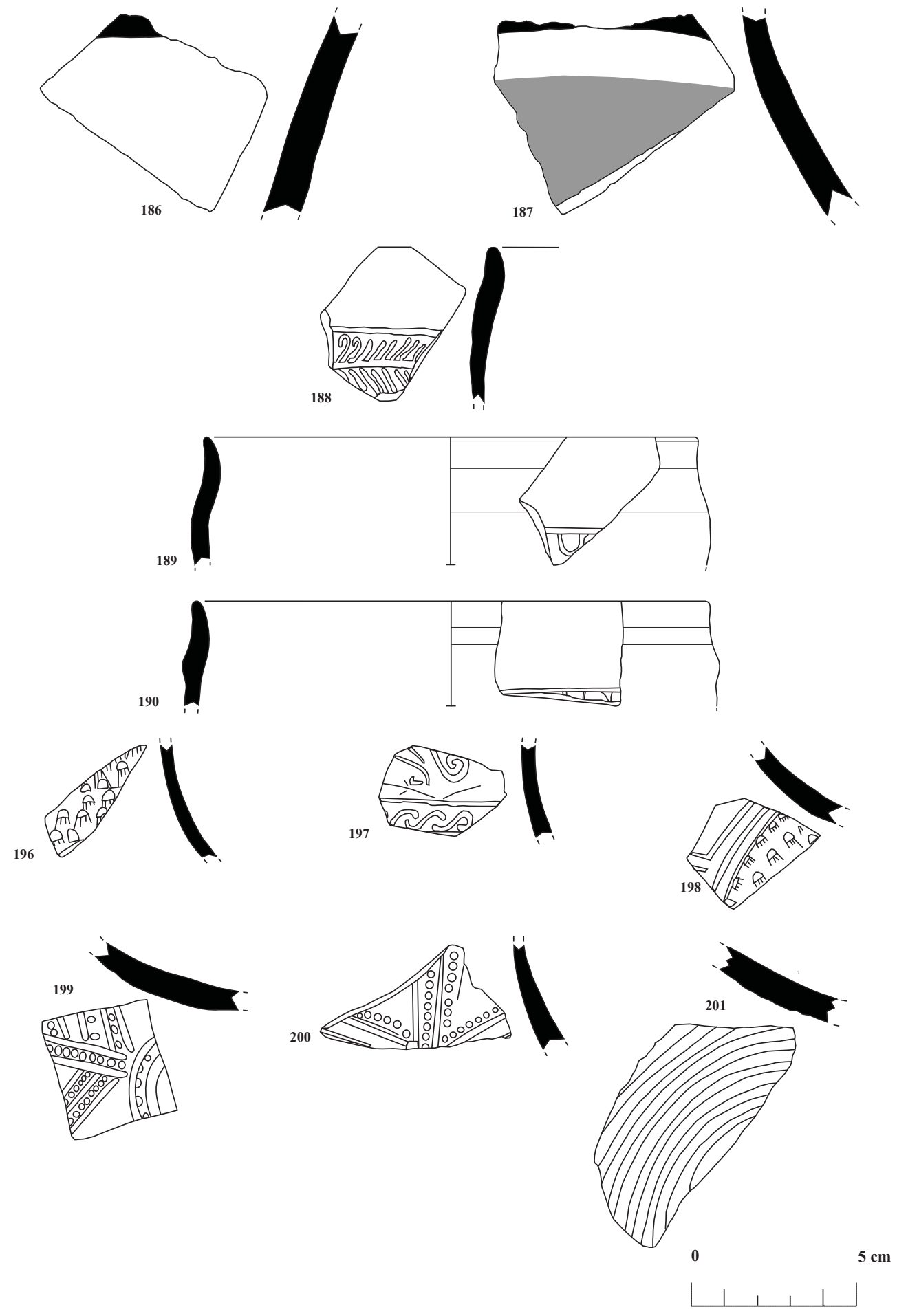

Pl. 6: Nos. 186-187: Hellenistic painted ware, body fragments of closed forms; nos. 188201: Hellenistic relief ware; nos. 188-190: Rim fragments of a bowl form; and nos. 196-201: Body fragments of open forms. 

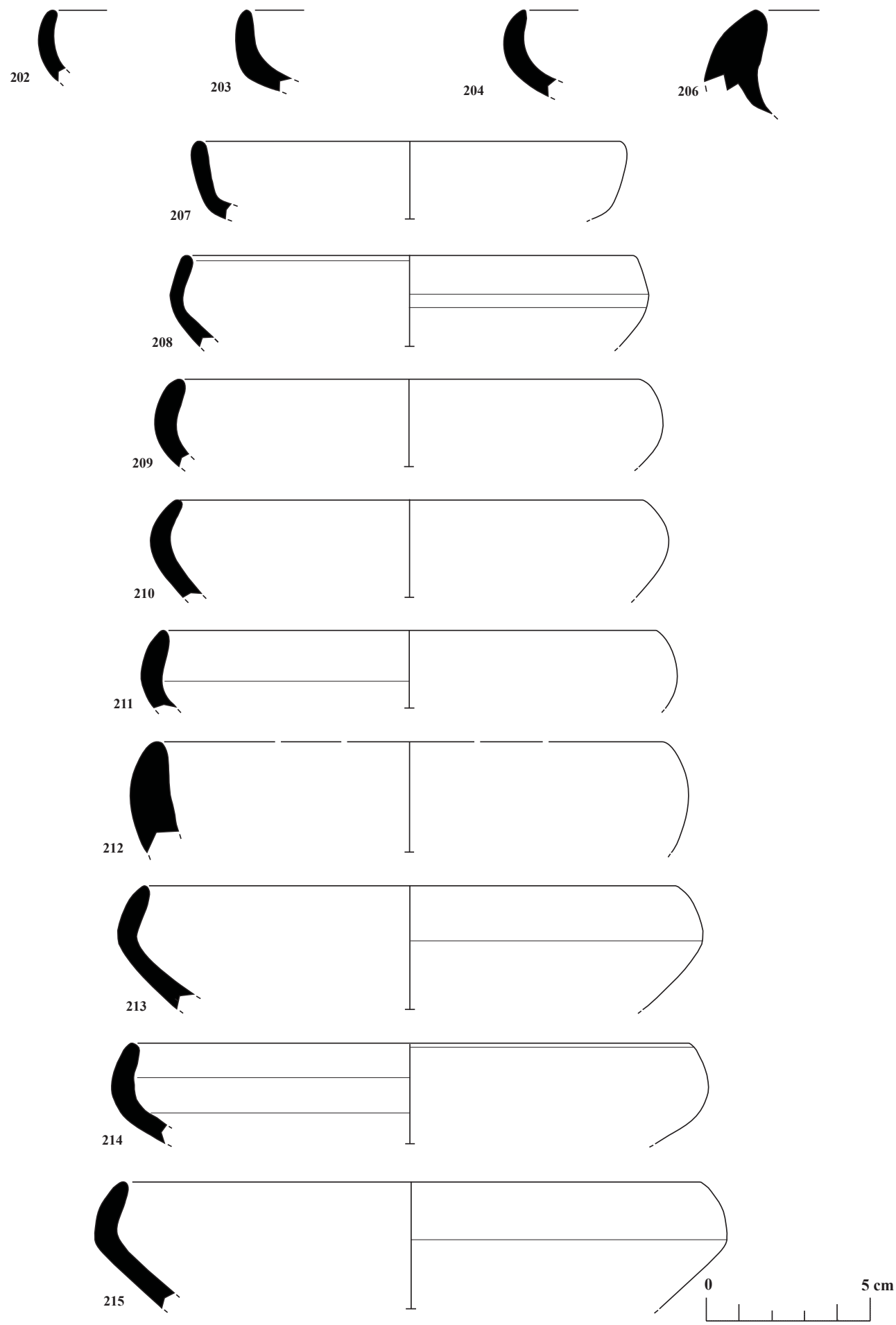

PI. 7: Nos. 202-215: Hellenistic burnished ware, rim fragments of a bowl form. 

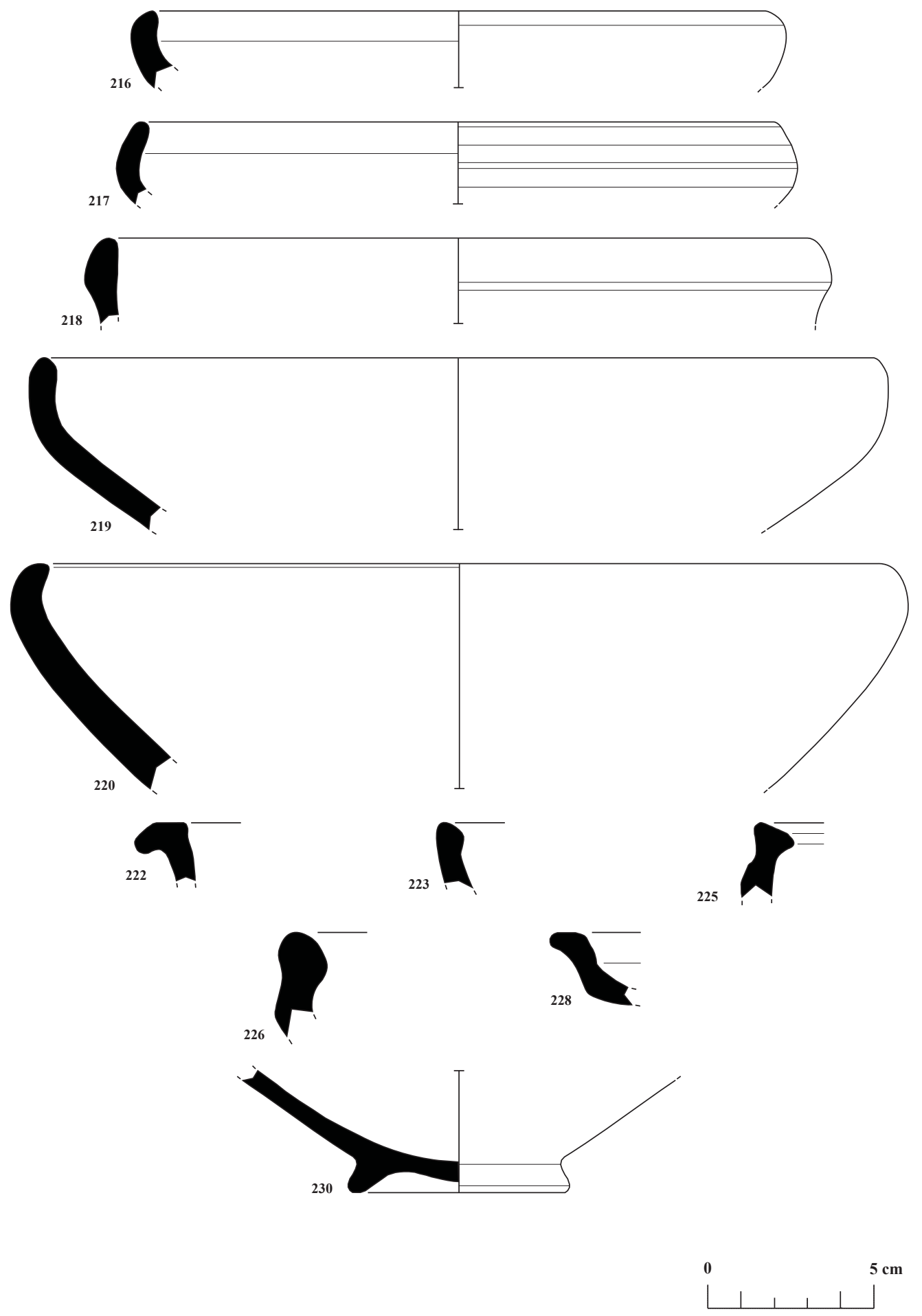

PI. 8: Nos. 216-230: Hellenistic burnished ware; nos. 216-220: Rim fragments of a bowl form; nos. 222-228: Other rim fragments; and no. 230: A base fragment of open forms. 

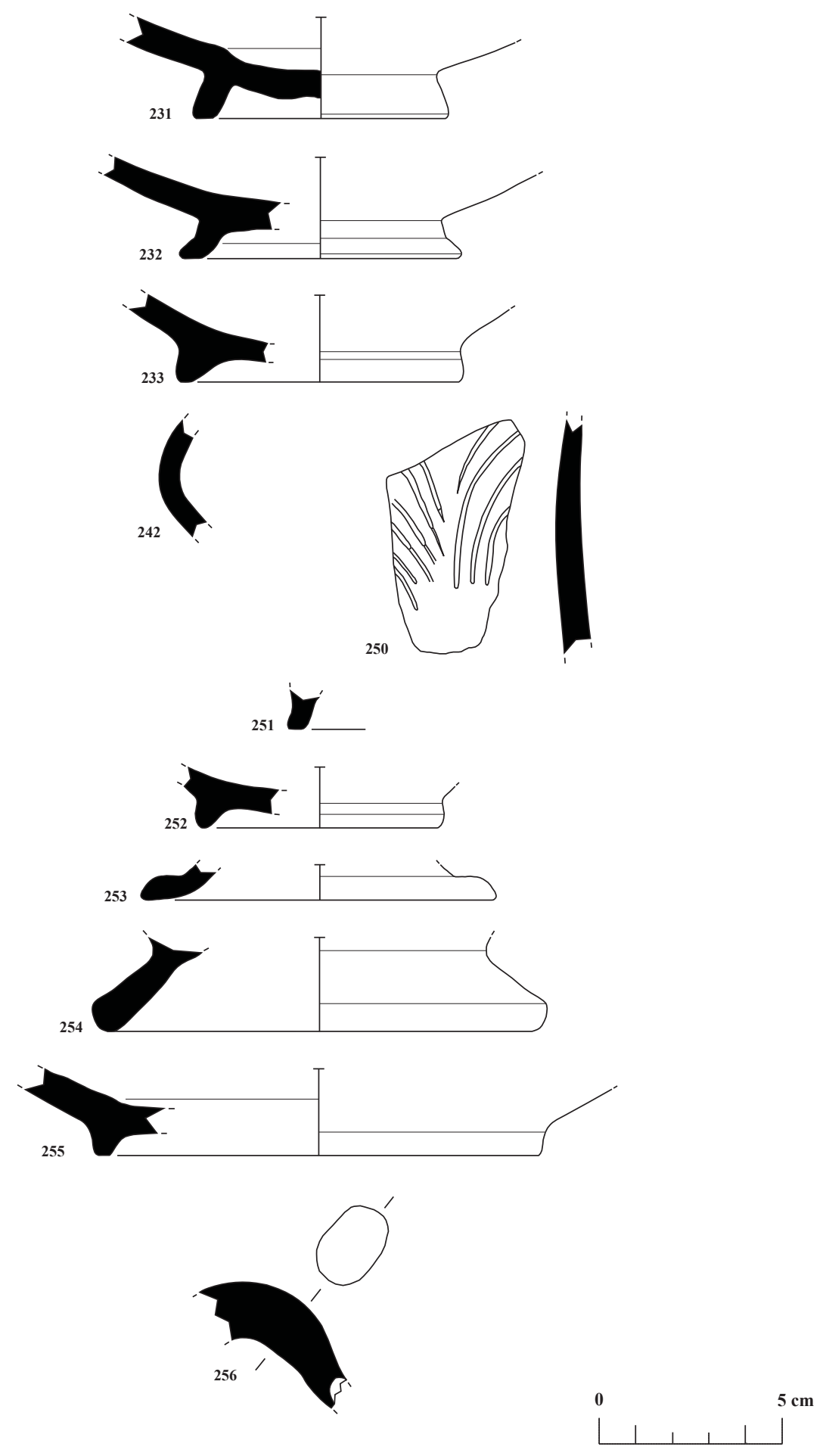

Pl. 9: Nos. 231-256: Hellenistic burnished ware; nos. 231-233: Base fragments of open forms; no. 242: A body fragment of open forms; no. 250: A body fragment of a rhyton; nos. 251-255: Base fragments of closed forms; and no. 256: A handle fragment of closed forms. 

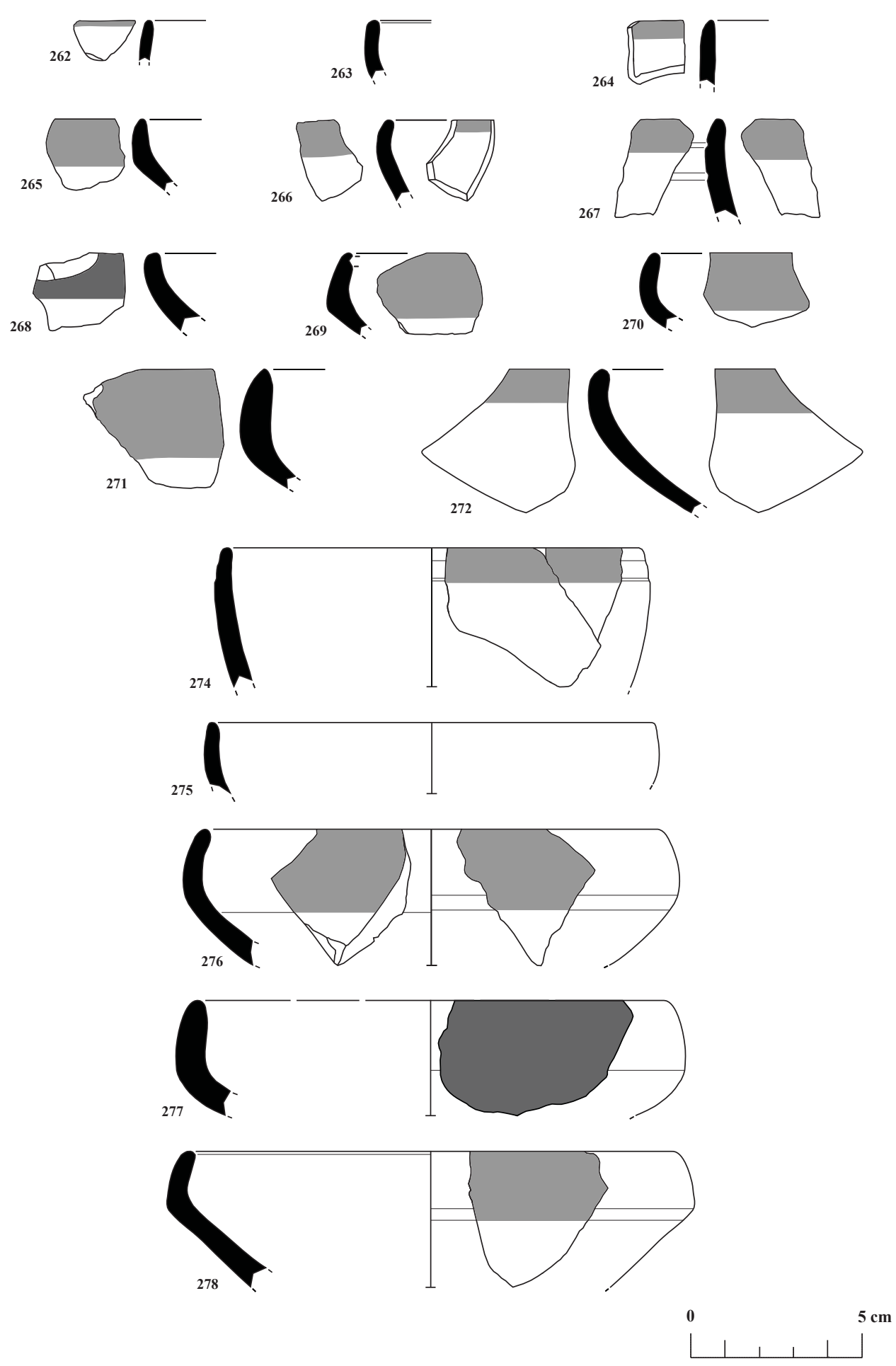

Pl. 10: Nos. 262-278: Red-painted Kepez group, bowl form 1. 

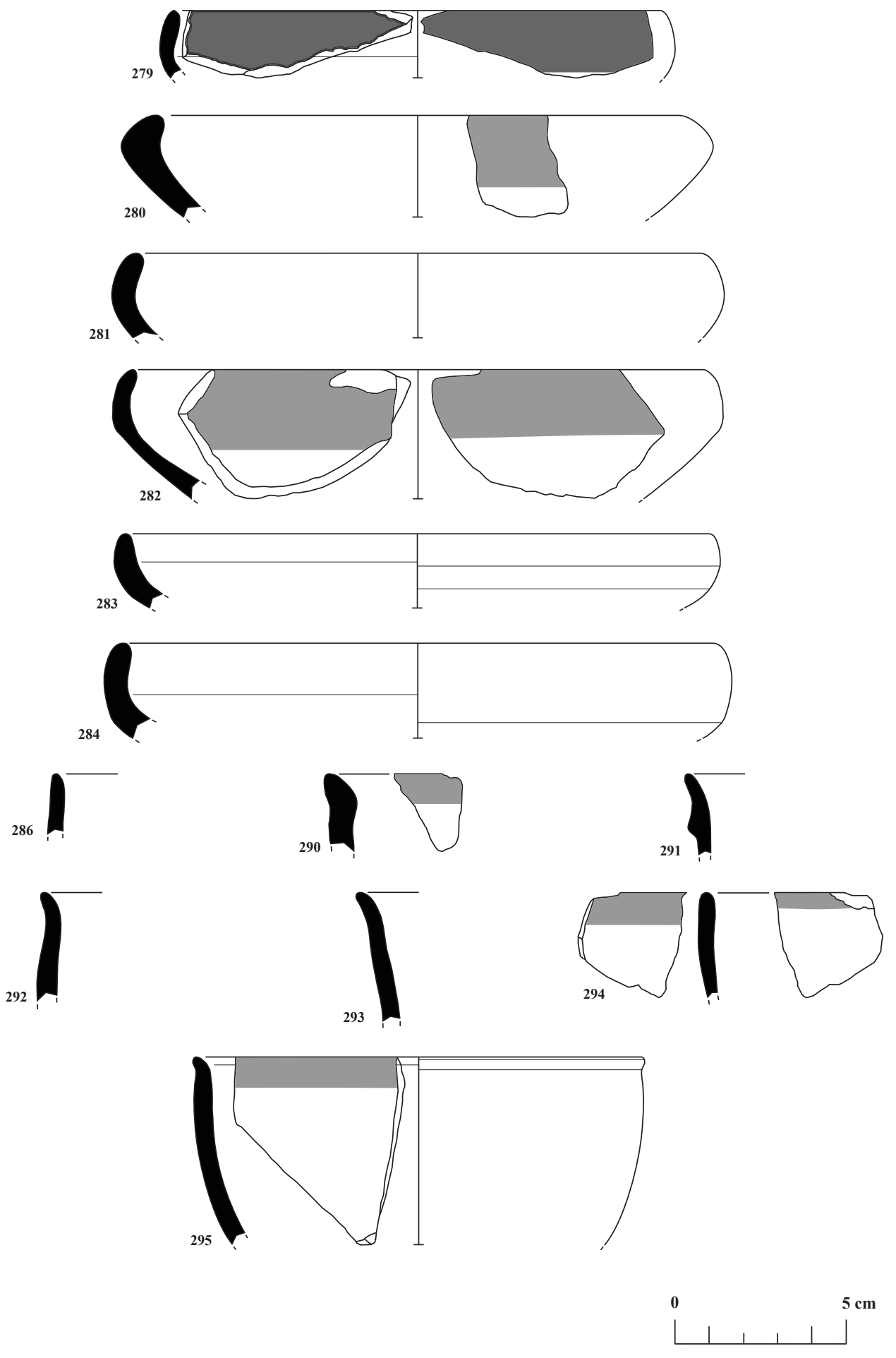

Pl. 11: Nos. 279-295: Red-painted Kepez group; nos. 279-284: Bowl form 1; and nos. 286295: Bowl form 2. 

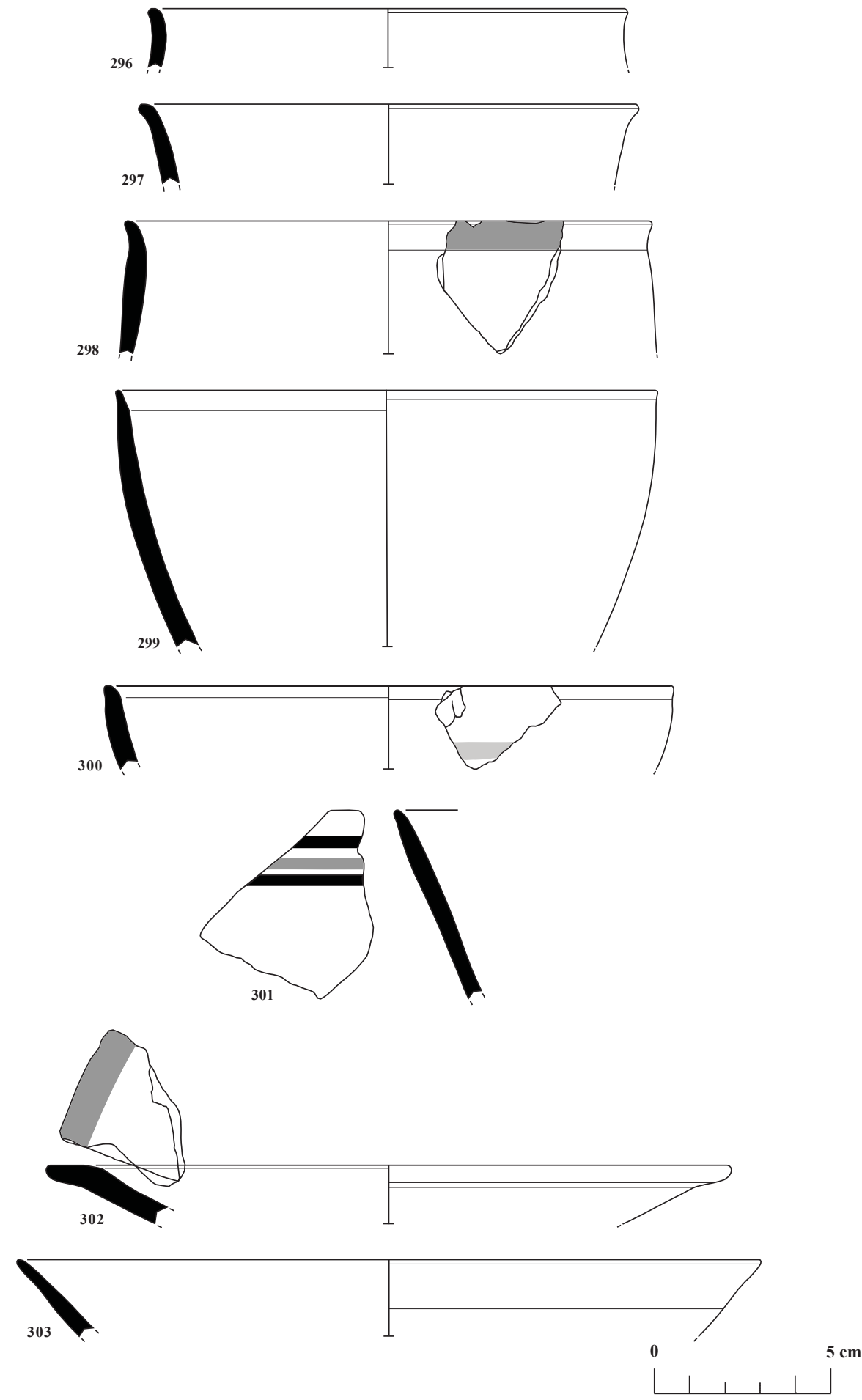

PI. 12: Nos. 296-303: Red-painted Kepez group; nos. 296-300: Bowl form 2; and nos. 301303: Dish form 1. 

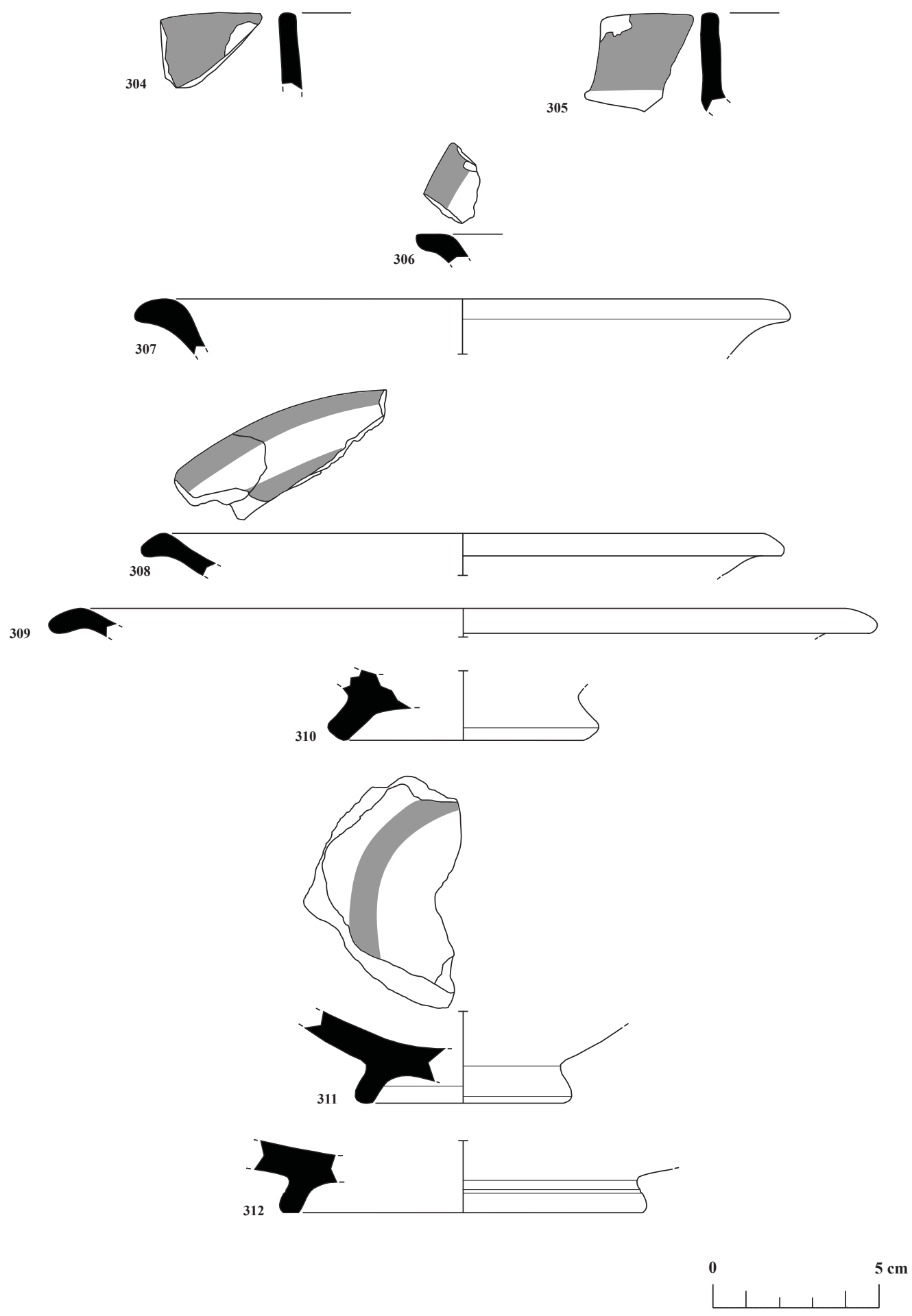

PI. 13: Nos. 304-312: Red-painted Kepez group; nos. 304-305: Dish form 2; nos. 306-309: Plate; and nos. 310-312: Base fragments of open forms. 

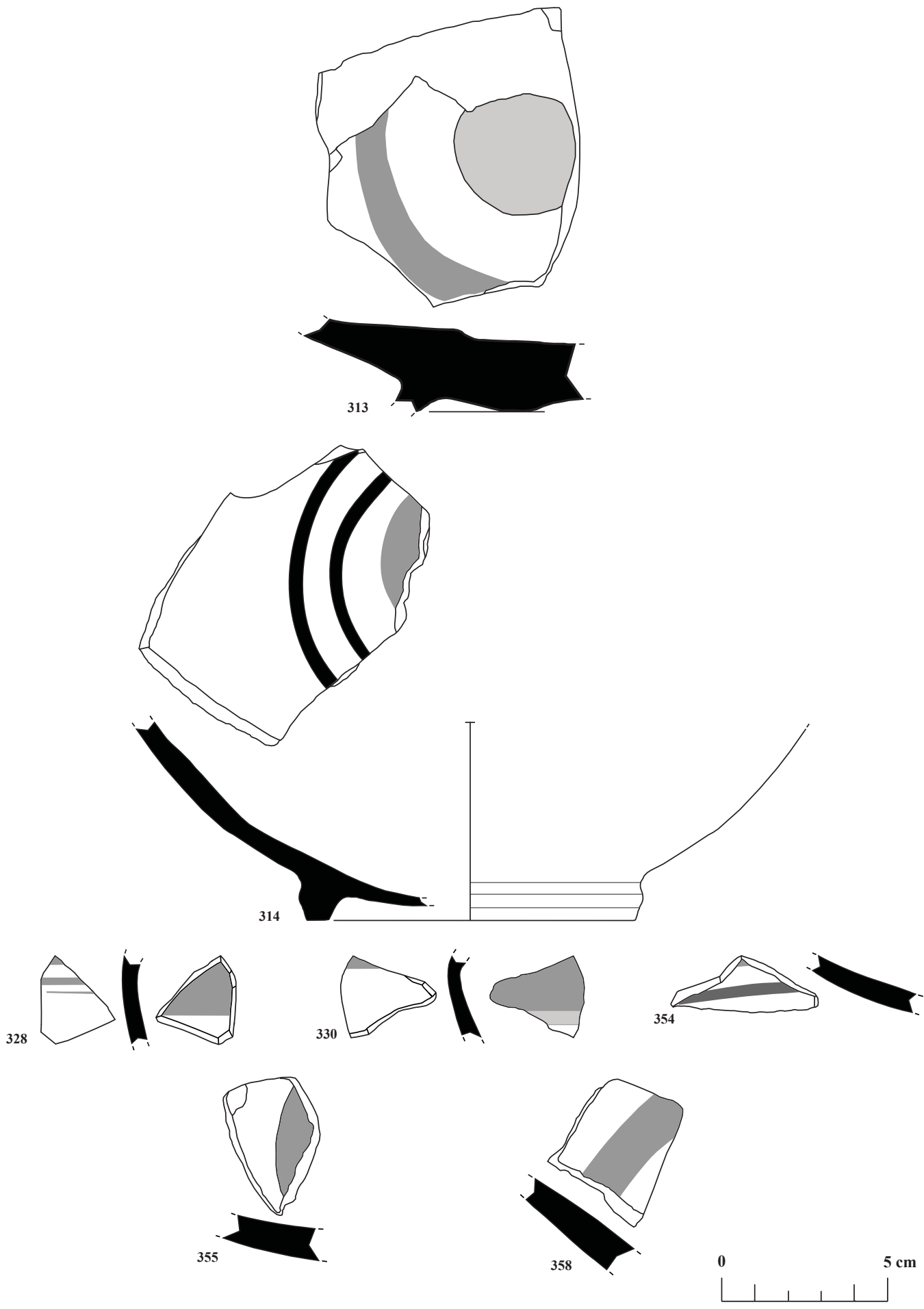

Pl. 14: Nos. 313-358: Red-painted Kepez group; nos. 313-314: Base fragments of open forms; and nos. 328- 358: Body fragments of open forms. 

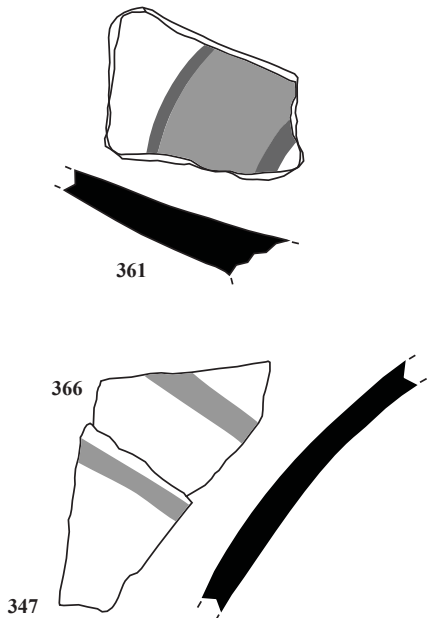
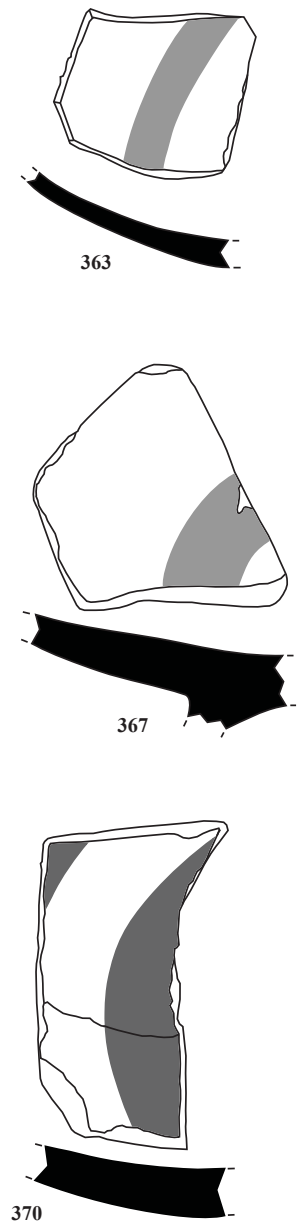
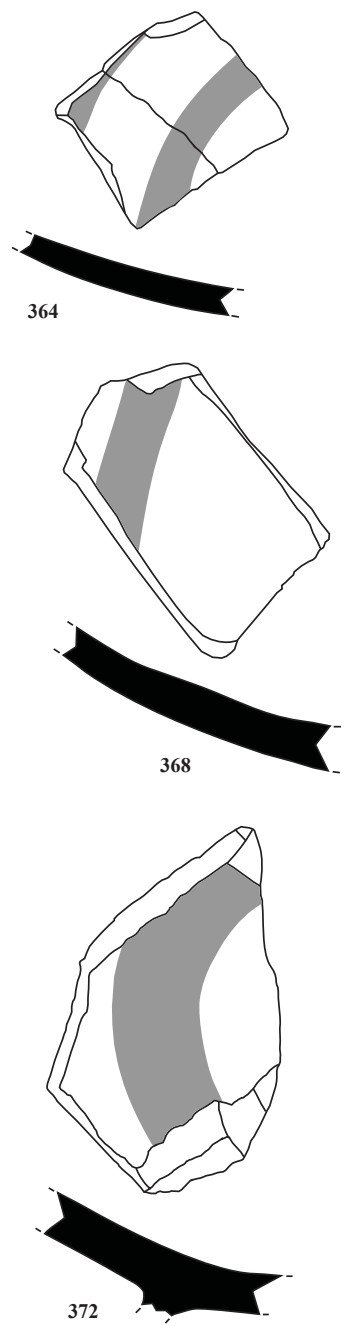
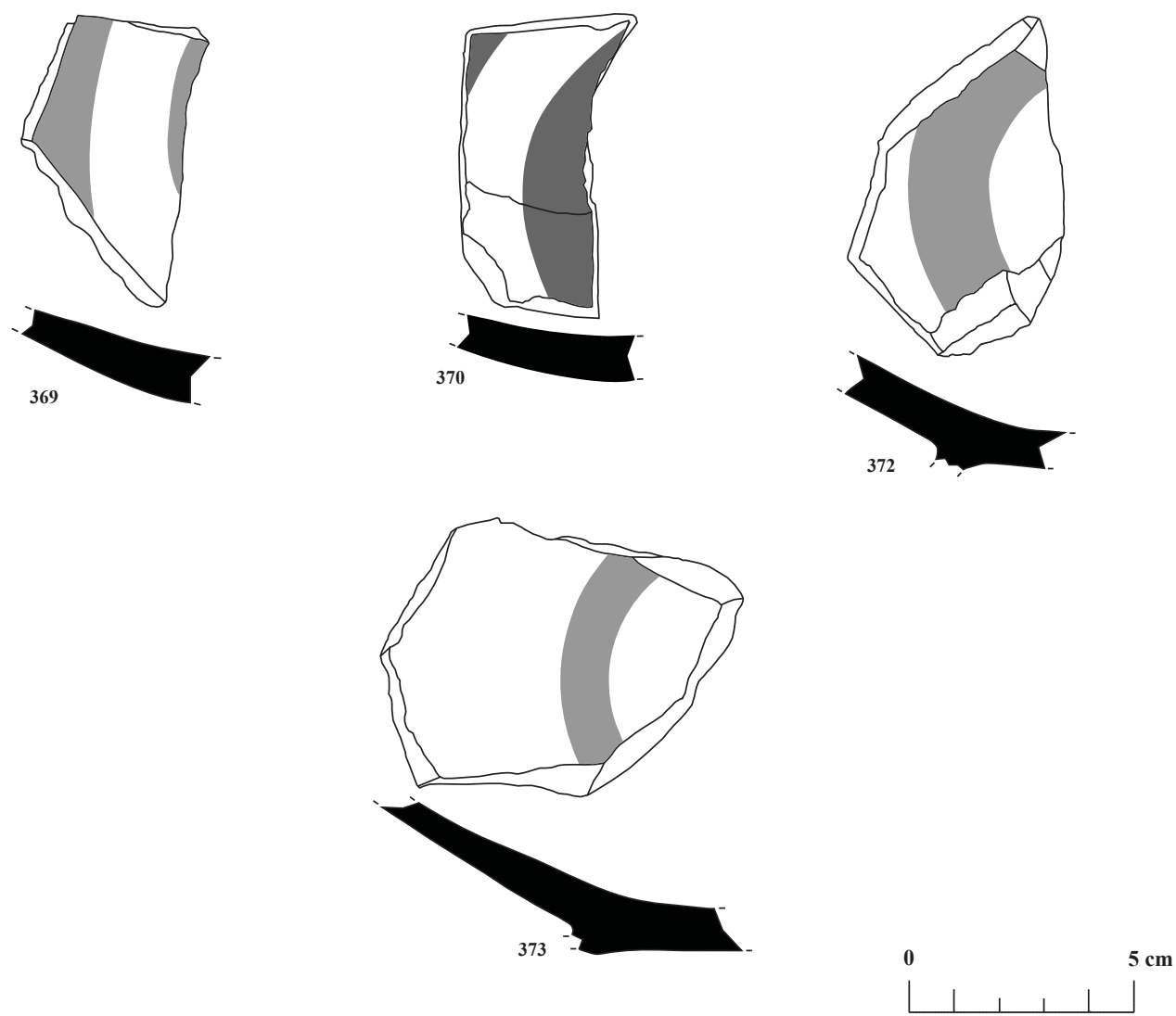

Pl. 15: Nos. 361-373: Red-painted Kepez group, body fragments of open forms. 


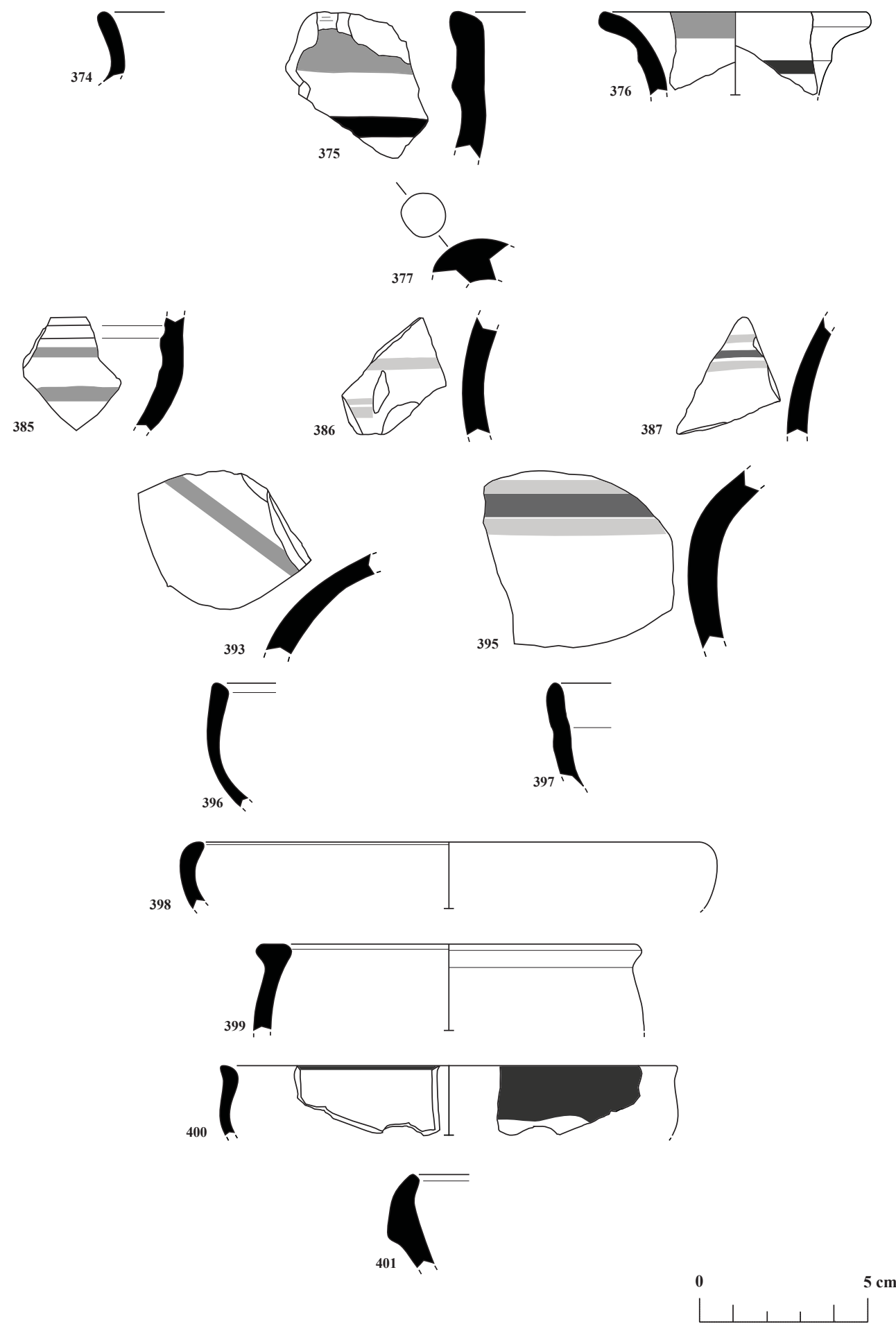

Pl. 16: Nos. 374-395: Red-painted Kepez group; nos. 374-376: Rim fragments of closed forms; no. 377: A handle fragment of closed forms; nos. 385-395: Body fragments of closed forms; nos. 396-401: Late Hellenistic-Early Roman grey ware; nos. 396-398: Bowl form 1; nos. 399-400: Bowl form 2; and no. 401: Dish form 1. 

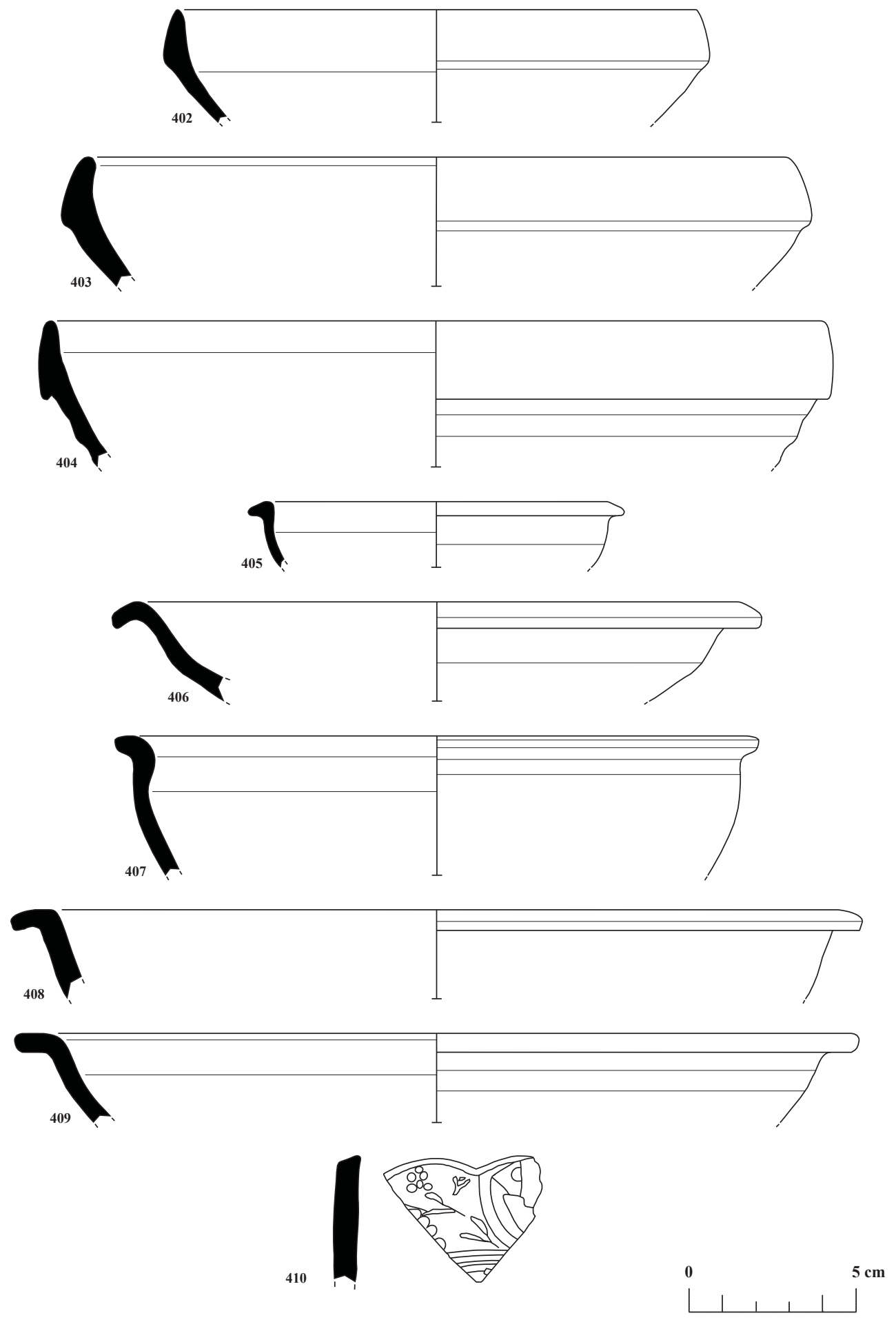

Pl. 17: Nos. 402-410: Late Hellenistic-Early Roman grey ware; nos. 402-404: Dish form 1; nos. 405-409: Dish form 2; and no. 410: A rim fragment of a plate. 


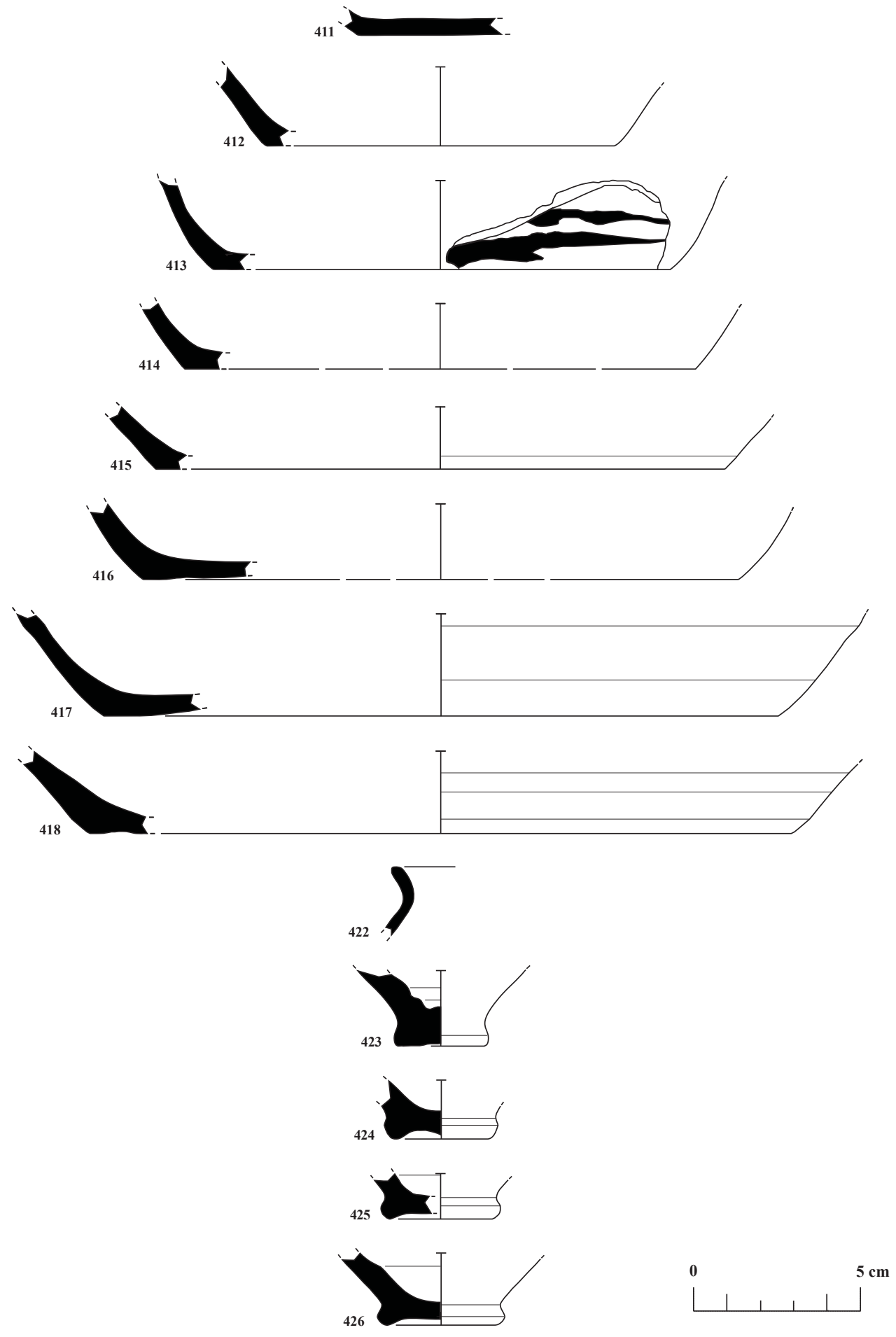

Pl. 18: Nos. 411- 426: Late Hellenistic-Early Roman grey ware; nos. 411-418: Base fragments of open forms; no. 422: A Rim fragment of a juglet; and nos. 423-426: Base fragments of closed forms. 

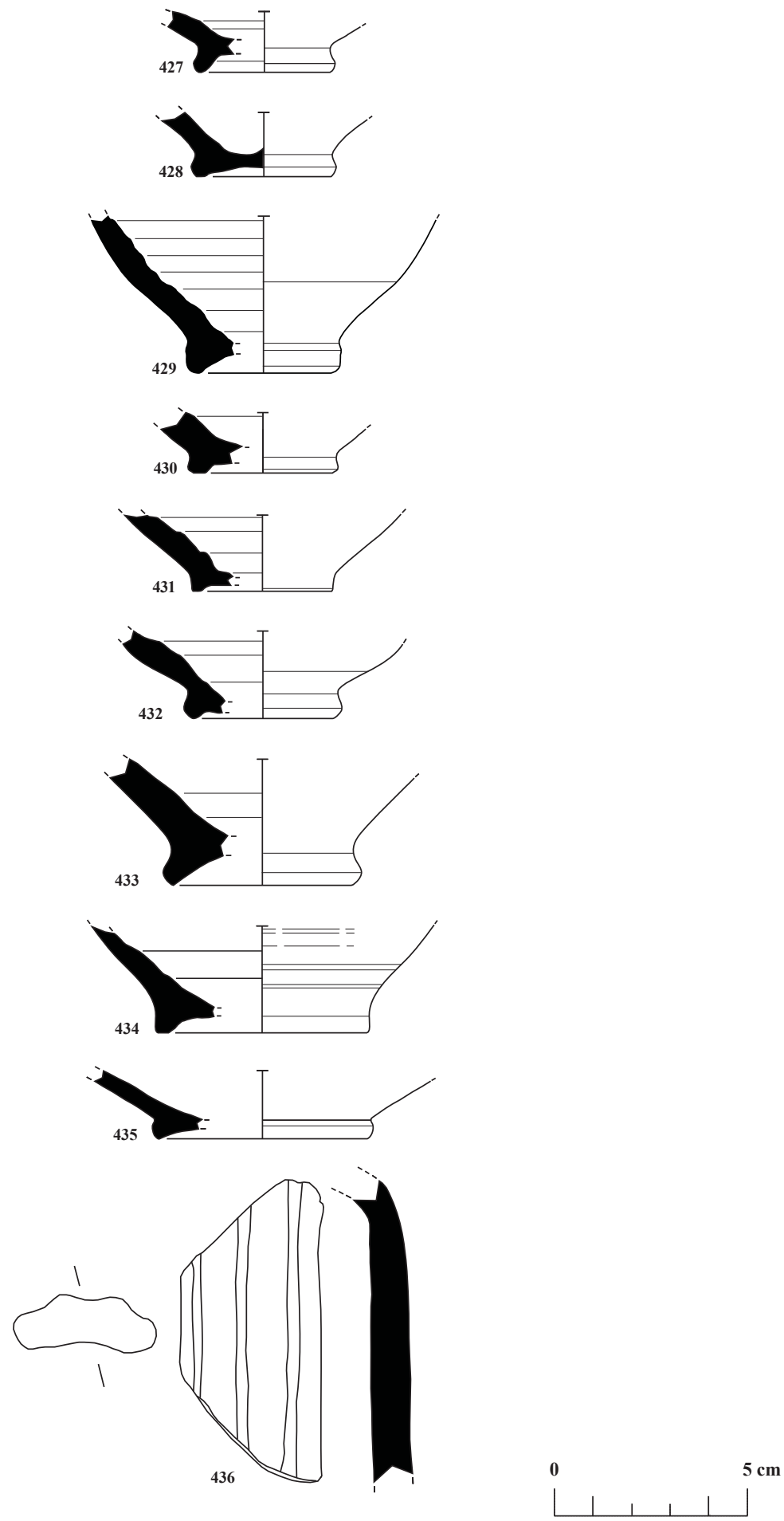

Pl. 19: Nos. 427-436: Late Hellenistic-Early Roman grey ware; nos. 427-435: Base fragments of closed forms; and no. 436: A handle fragment of closed forms. 

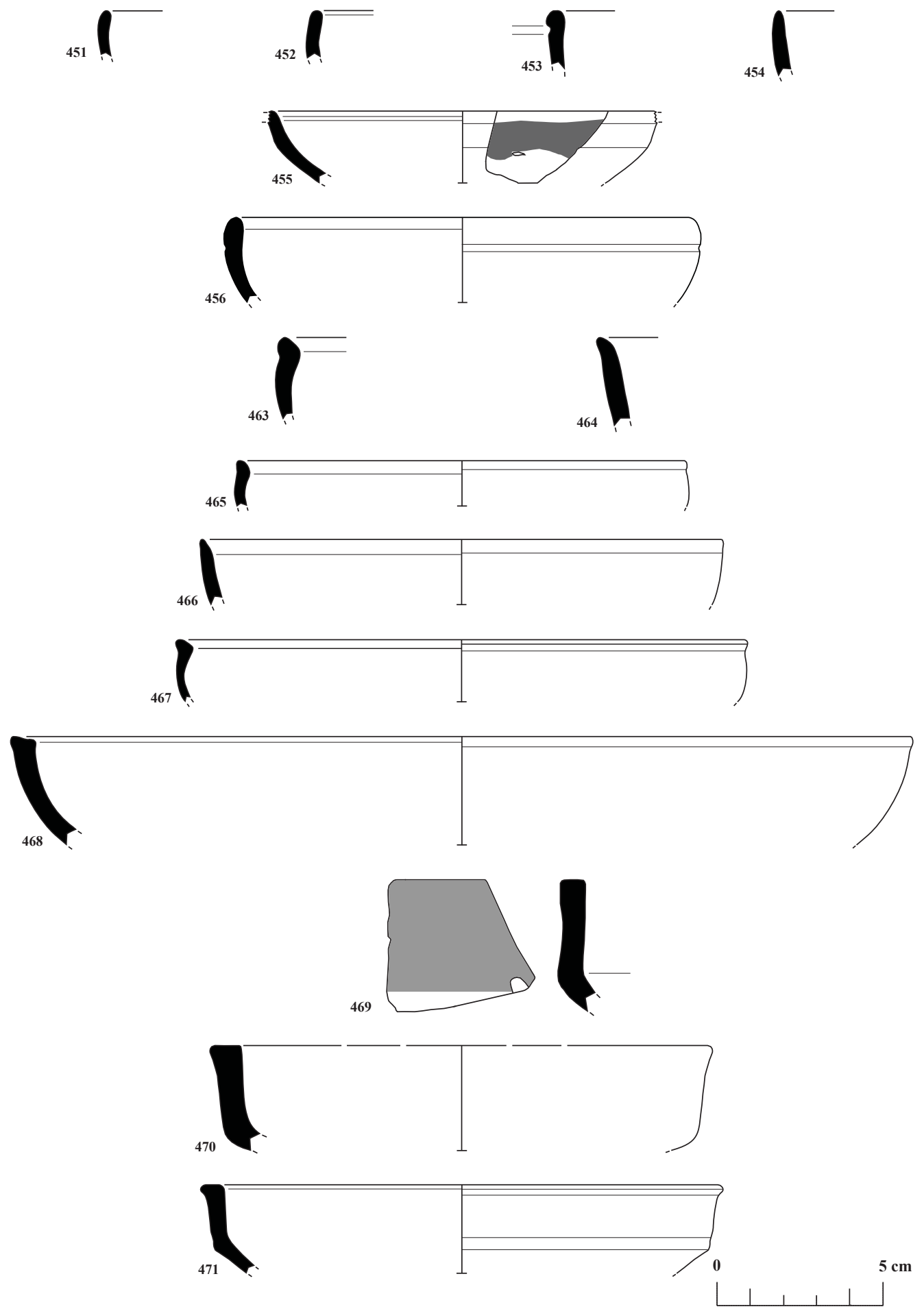

Pl. 20: Nos. 451-471: Late Hellenistic-Early Roman brown-slipped ware; nos. 451-456: Bowl form 1; nos. 463-468: Bowl form 2; and nos. 469-471: Dish. 


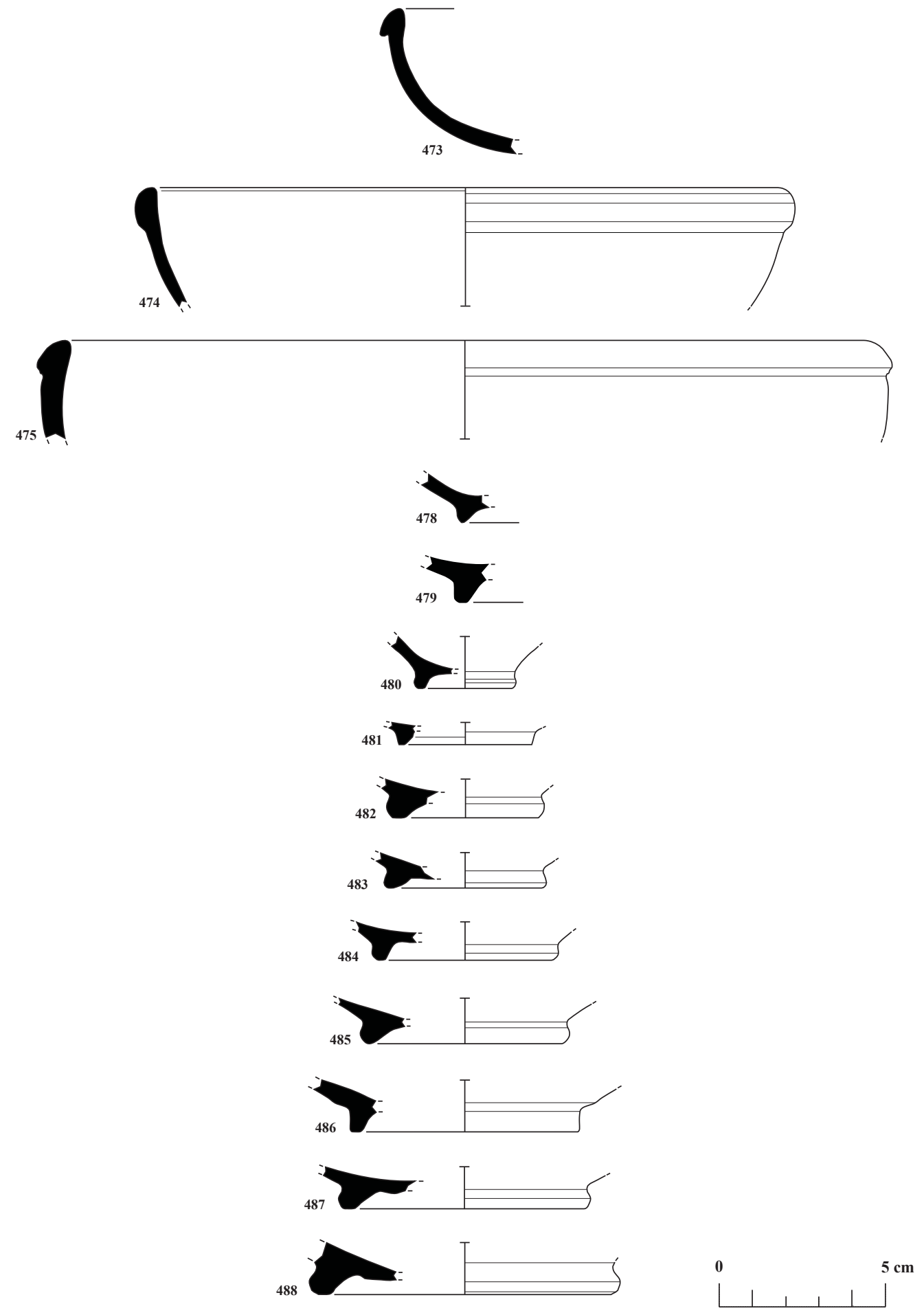

PI. 21: Nos. 473-488: Late Hellenistic-Early Roman brown-slipped ware; nos. 473-475: Other rim forms; and nos. 478-488: Base fragments of open forms. 

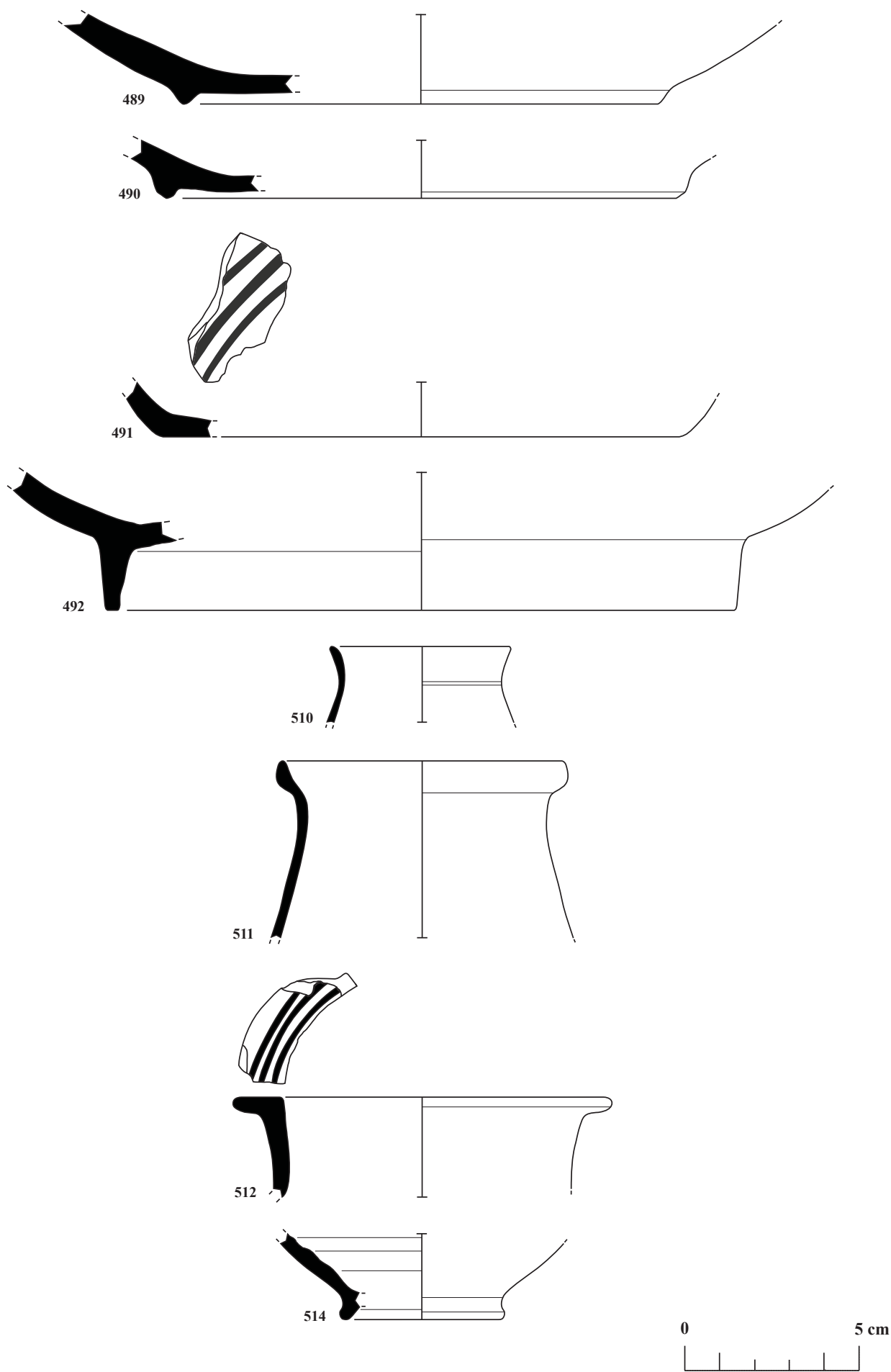

Pl. 22: Nos. 489-514: Late Hellenistic-Early Roman brown-slipped ware; nos. 489-492: Base fragments of open forms; nos. 510-512: Juglet; and no. 514: A base fragment of closed forms. 

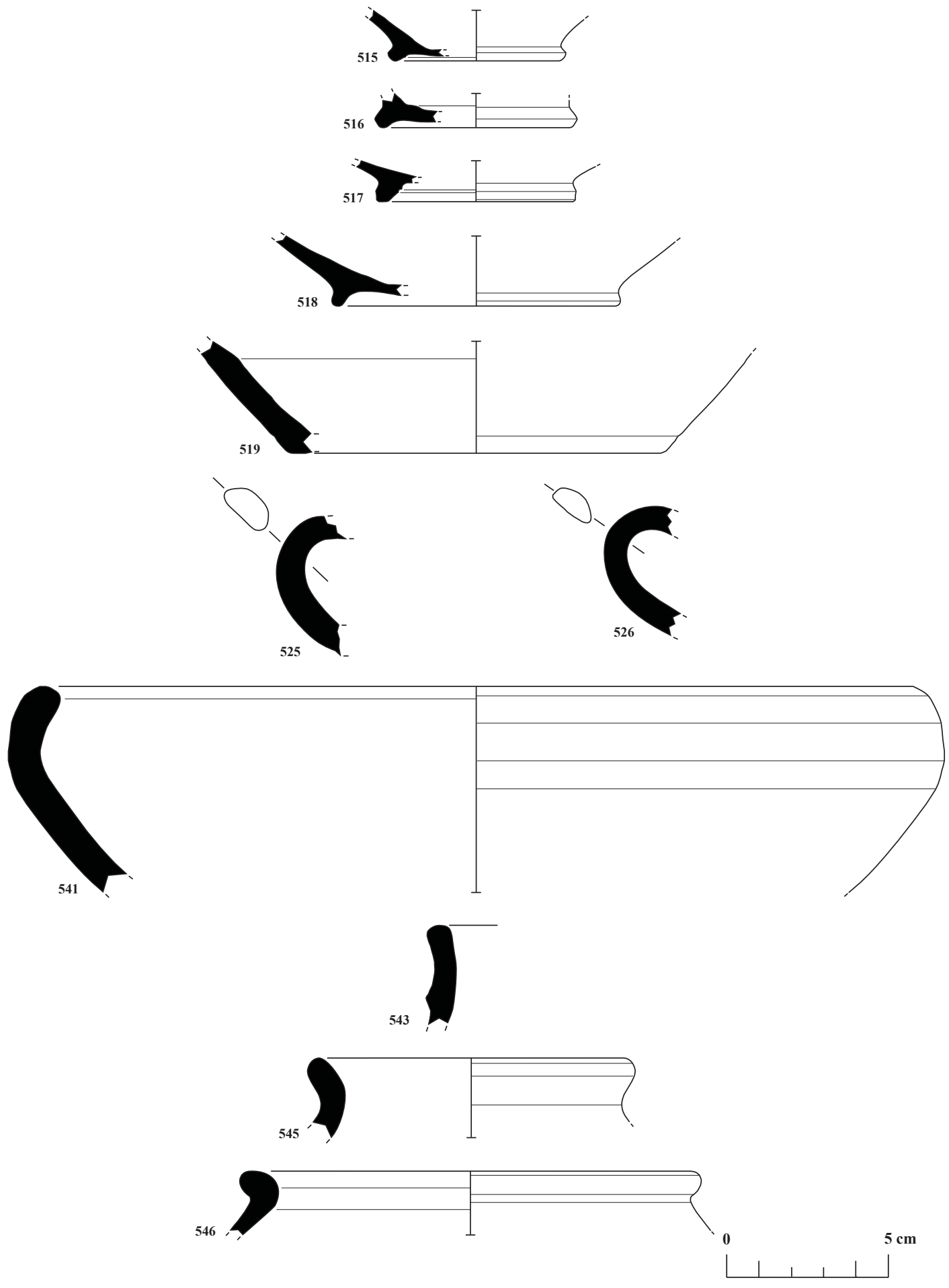

PI. 23: Nos. 515-526: Late Hellenistic-Early Roman brown-slipped ware; nos. 515-519: Base fragments of closed forms; nos. 525-526: Handle fragments of closed forms; nos. 541-546: Hellenistic Coarse Ware; no. 541: A rim fragment of open forms; and nos. 543-546: Rim fragments of closed forms. 

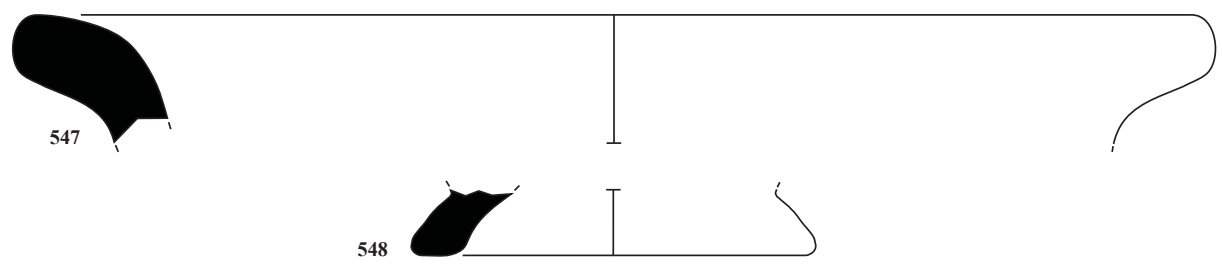

548
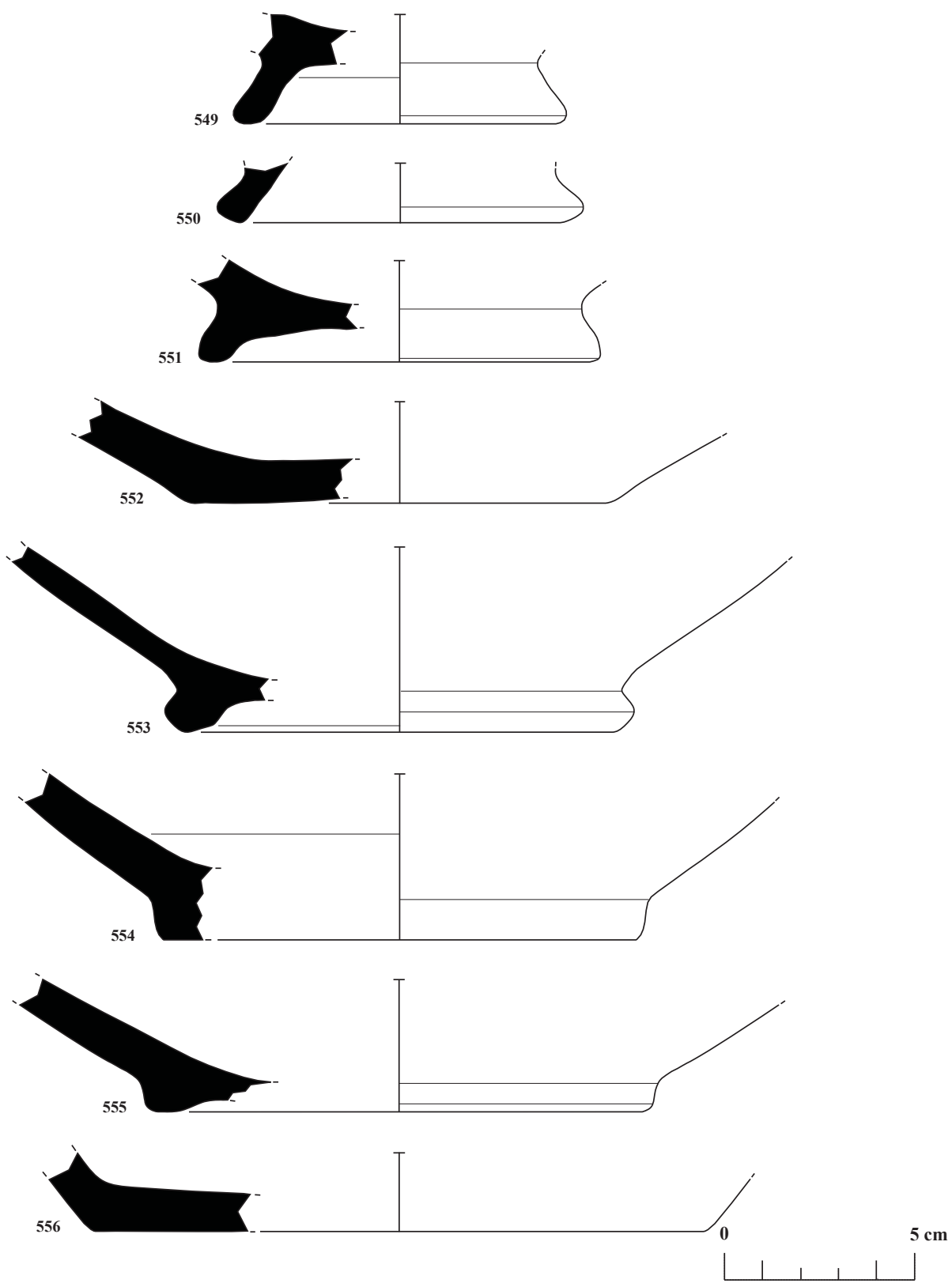

PI. 24: Nos. 547-556: Hellenistic coarse ware; no. 547: A rim fragment of closed forms; and nos. 548-556: Base fragments of closed forms. 

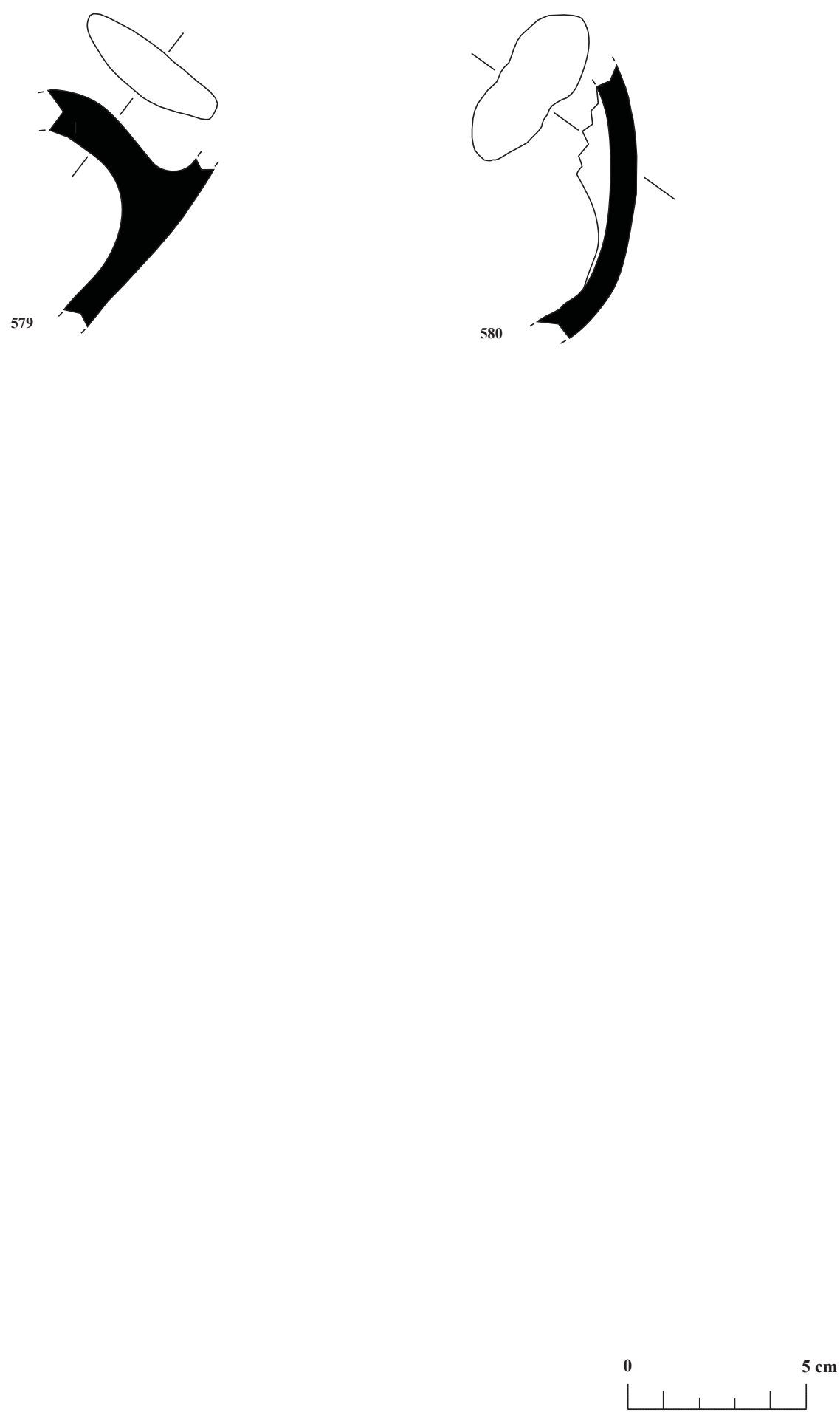

Pl. 25: Nos. 579-580: Hellenistic coarse ware, body fragments of closed forms. 

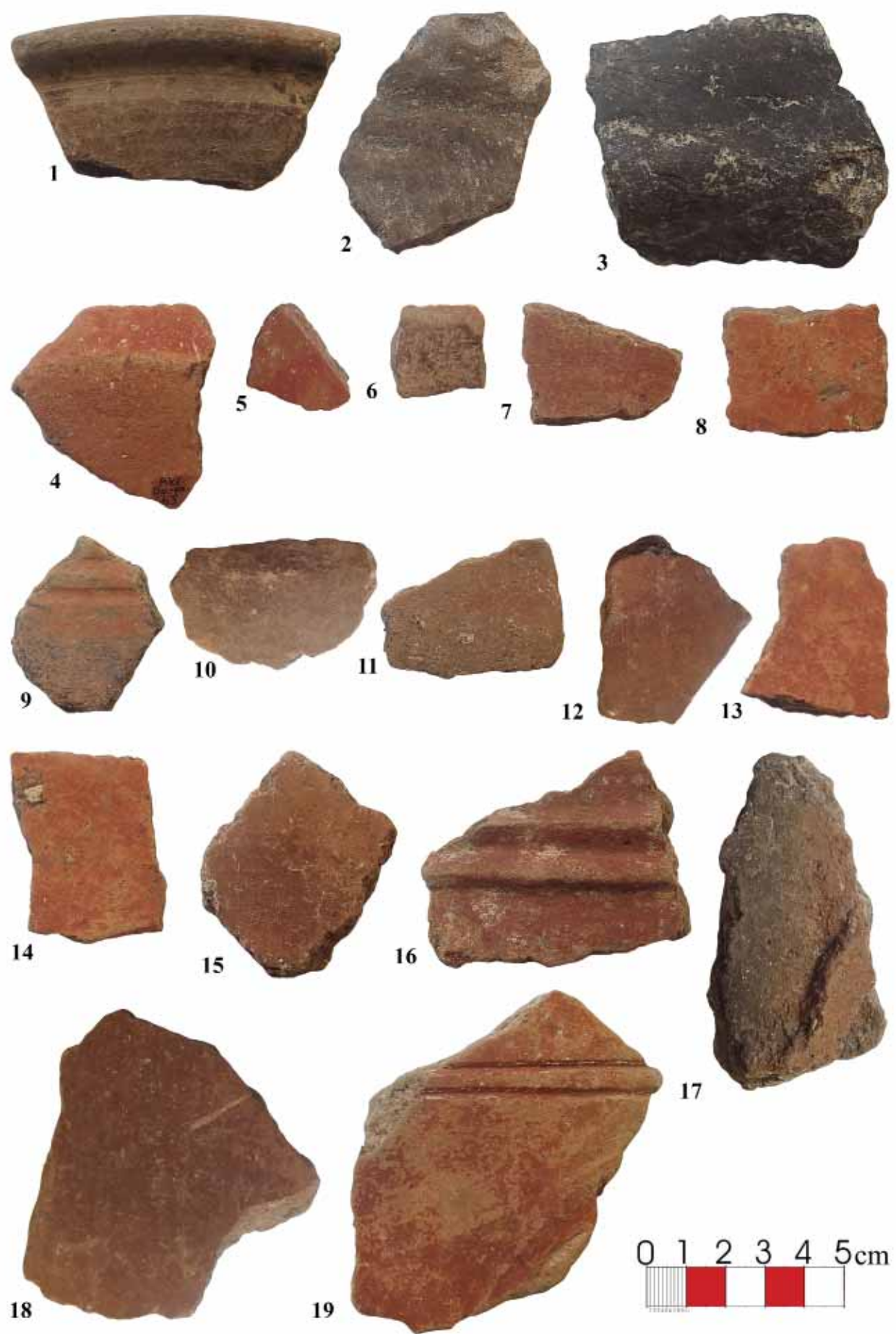

Pl. 26: Nos. 1-19: Pre-Iron Age pottery; nos. 1-2: Rim fragments; nos. 3-4: Base fragments of open forms; and nos. 5-19: Body fragments of open forms. 

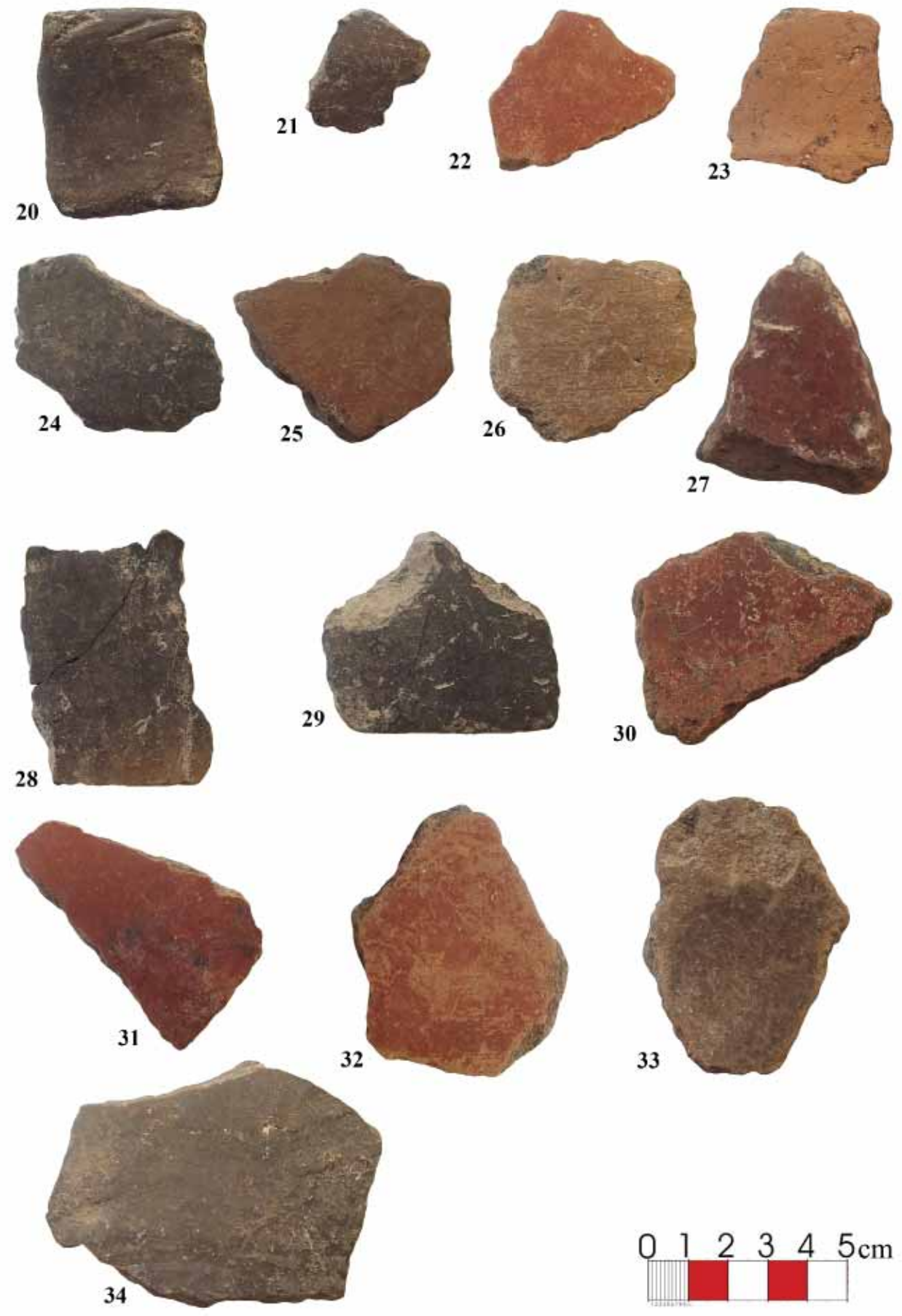

Pl. 27: Nos. 20-34: Pre-Iron Age pottery; no. 20: A rim fragment of closed forms; and nos. 2134: Body fragments of closed forms. 

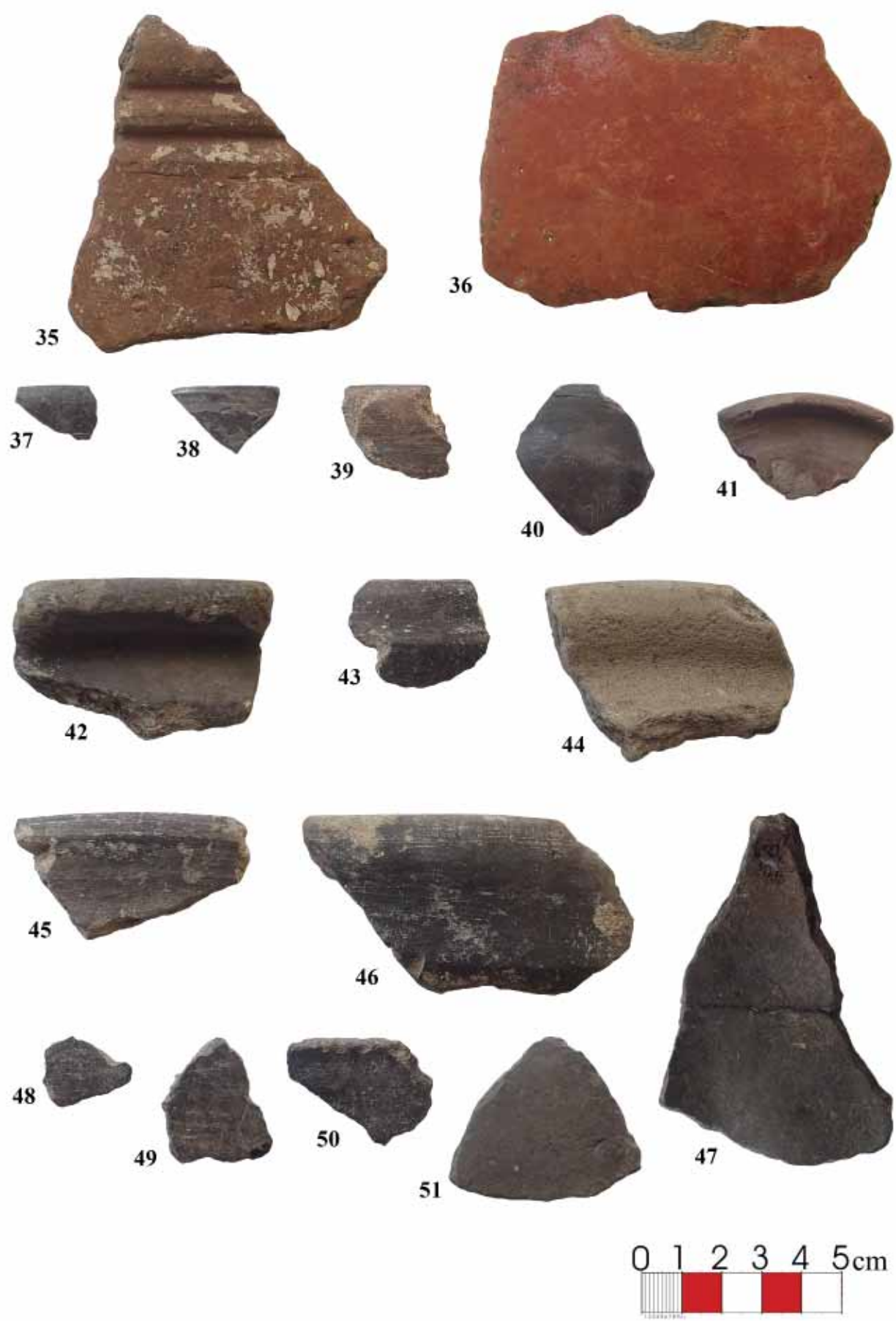

Pl. 28: Nos. 35-36: Pre-Iron Age pottery, body fragments of closed forms; nos. 37-51: Iron Age grey ware; nos. 37-46: Rim fragments of open forms; no. 47: A base fragment of open forms; and nos. 48-51: Body fragments of open forms. 


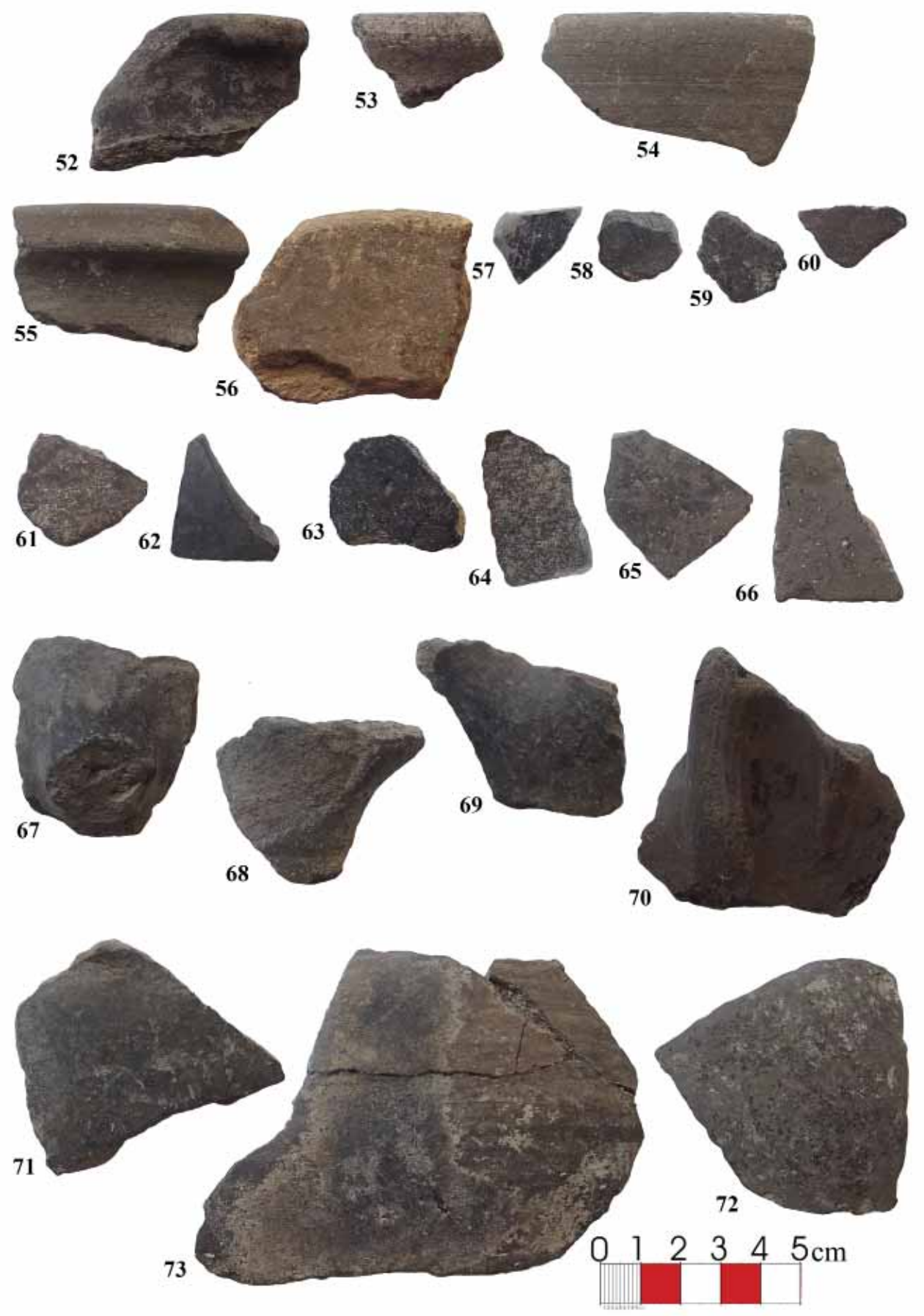

Pl. 29: Nos. 52-73: Iron Age grey ware; nos. 52-55: Rim fragments of closed forms; no. 56: A base fragment of closed forms; and nos. 57-73: Body fragments of closed forms. 

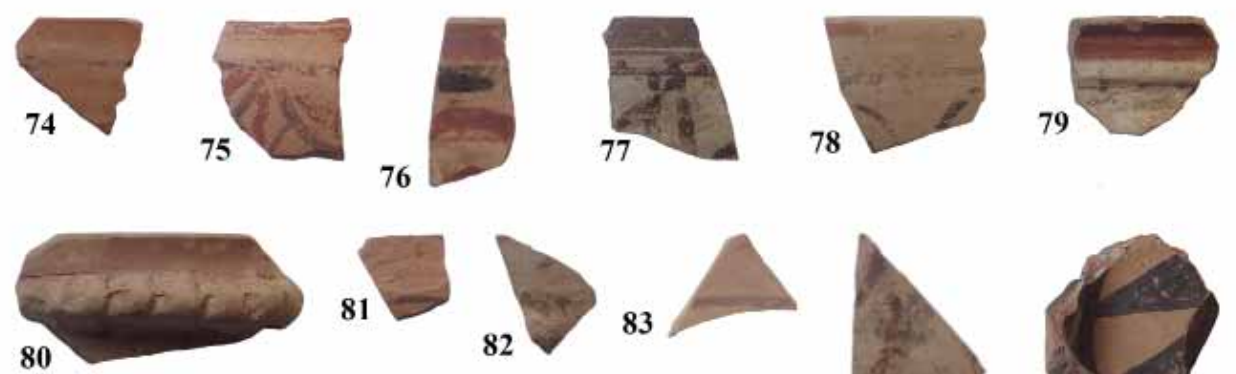

84
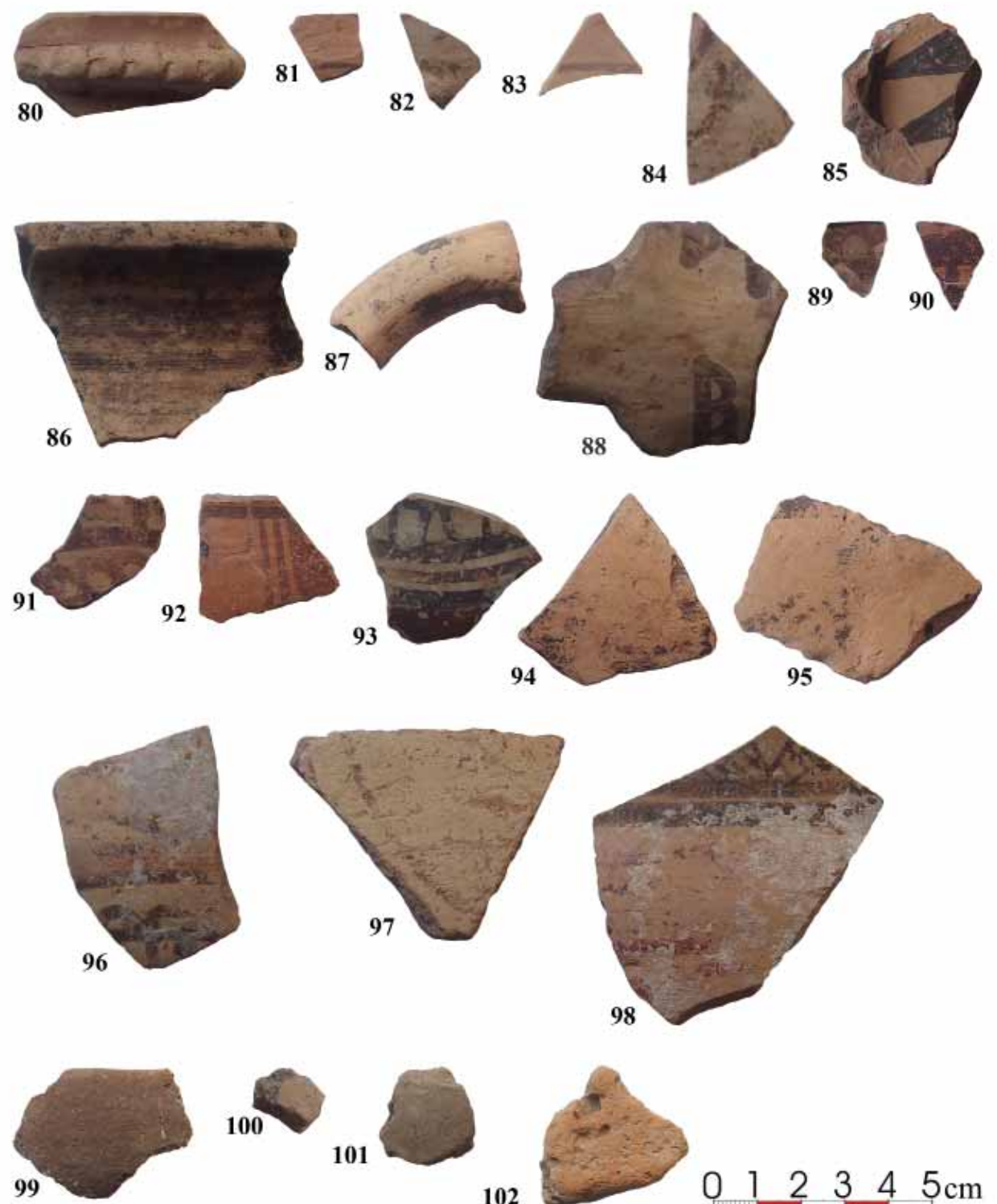

102
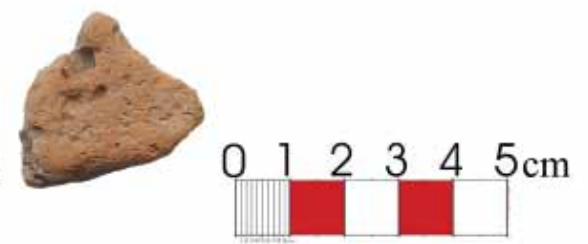

Pl. 30: Nos. 74-98: Iron Age painted ware; nos. 74-80: Rim fragments of a bowl form; nos. 81-85: Body fragments of open forms; no. 86: A rim fragment of closed forms; nos. 87-88: Handle fragments of closed forms; nos. 89-98: Body fragments of closed forms; nos. 99-102: Iron Age coarse ware; no. 99: A rim fragment of open forms; and nos. 100-102: Body fragments of open forms. 

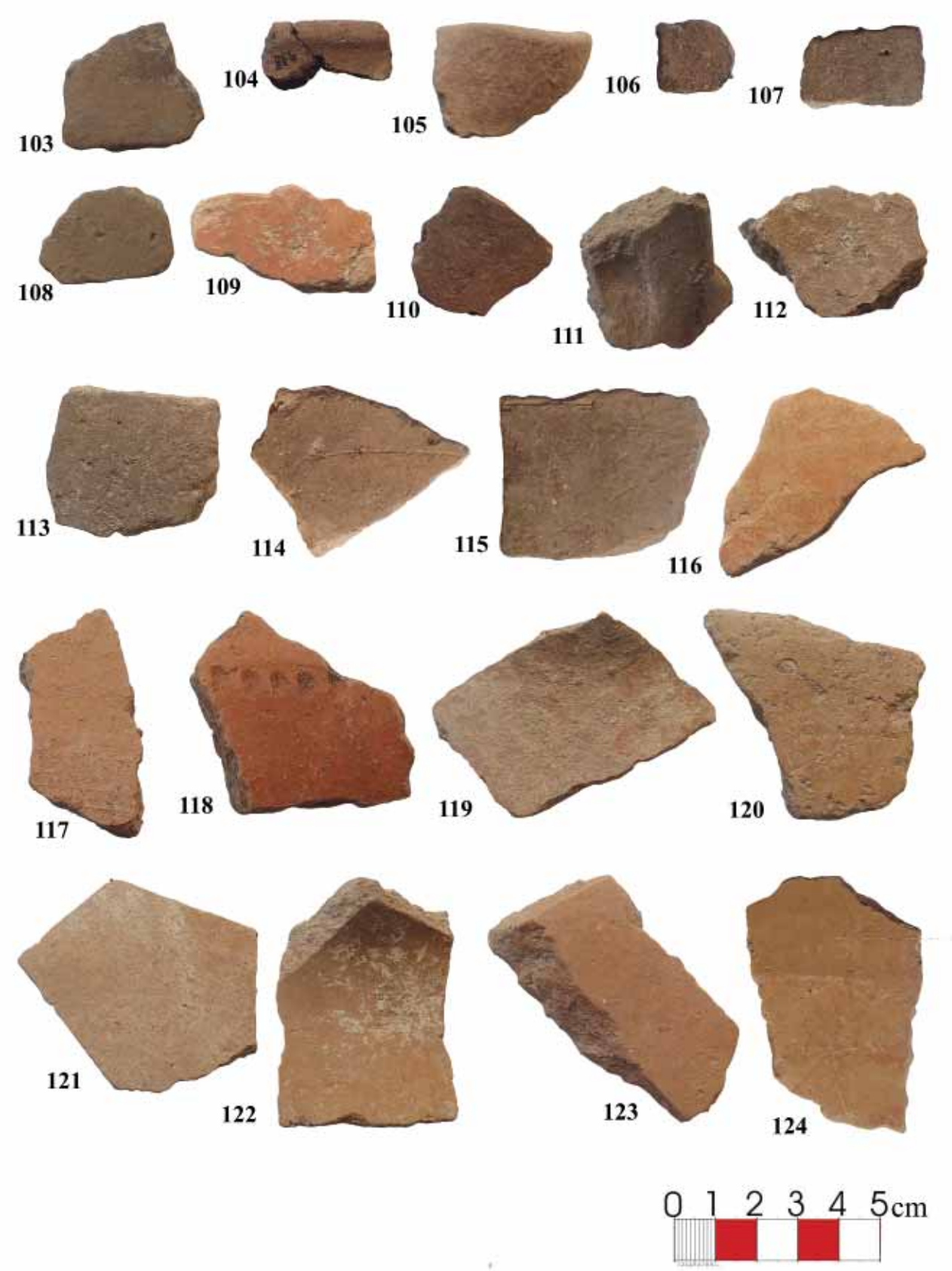

Pl. 31: Nos. 103-124: Iron Age coarse ware; no. 103: A body fragment of open forms; nos. 104-105: Rim fragments of closed forms; and nos. 106-124: Body fragments of closed forms. 


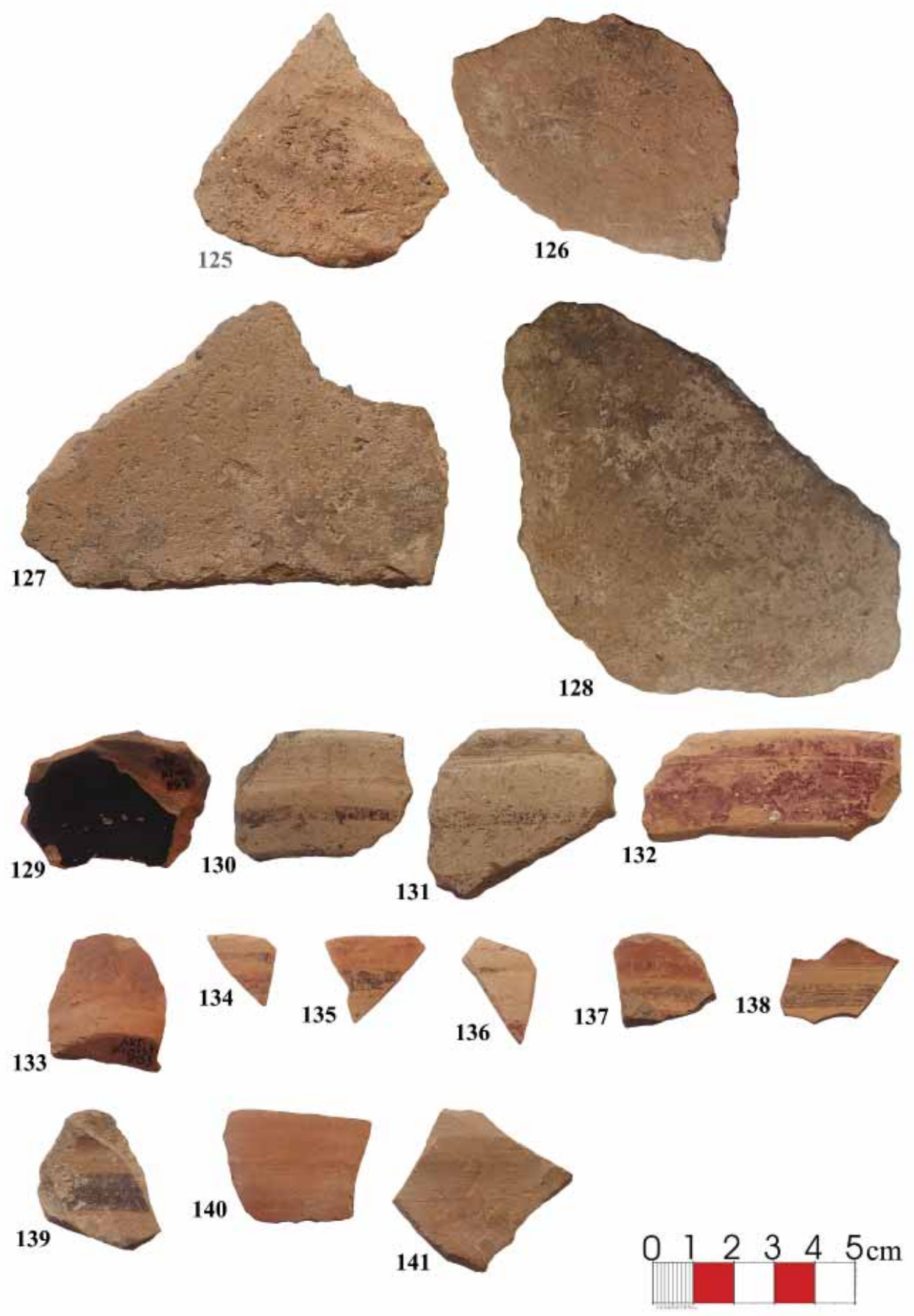

Pl. 32: Nos. 125-128: Iron Age coarse ware, body fragments of closed forms; no. 129: A Pontic skyphos fragment; nos. 130-141: Hellenistic Painted Ware; nos. 130-132: Rim fragments of open forms; no. 133: A base fragment of open forms; and nos. 134-141: Body fragments of open forms. 

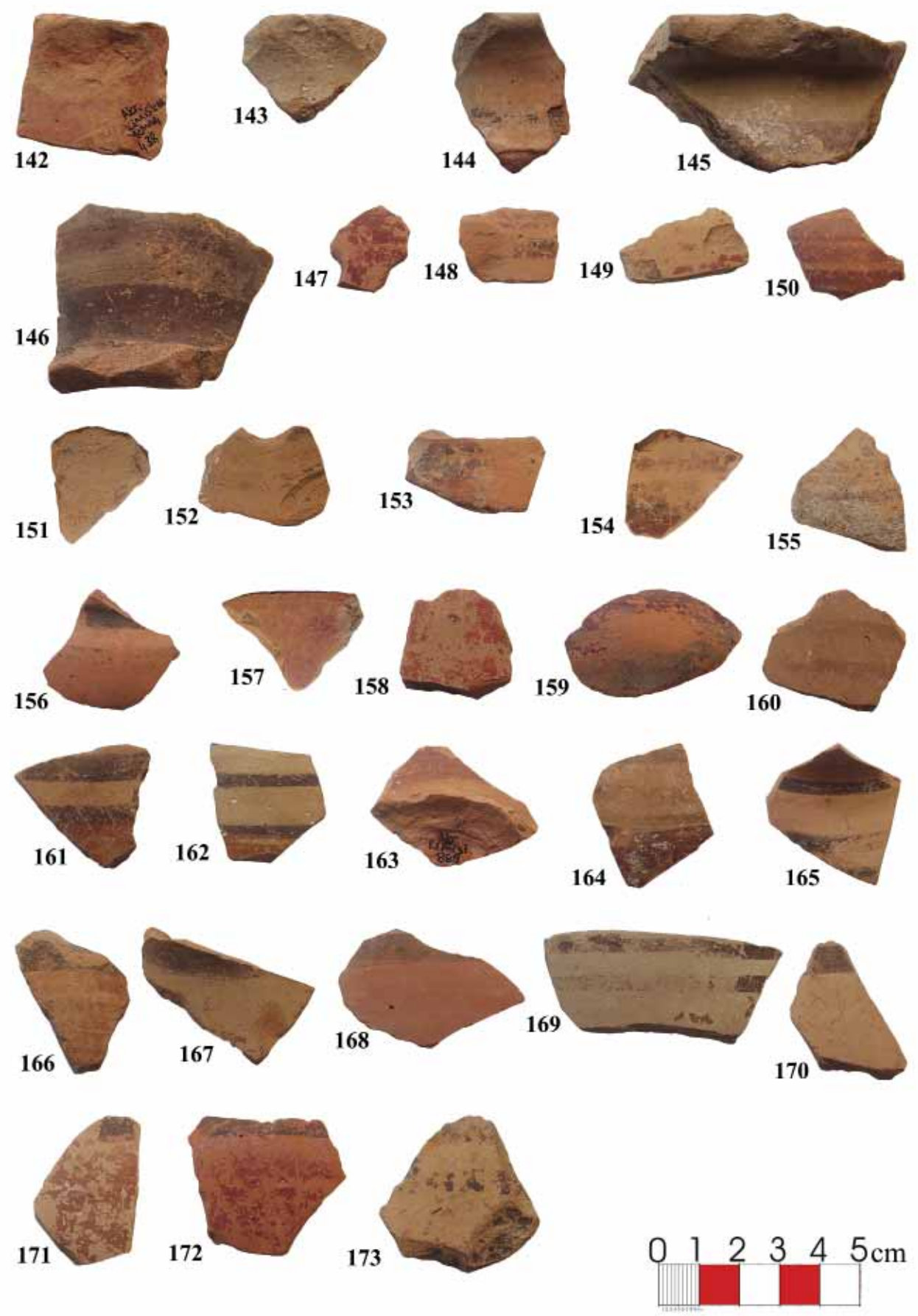

Pl. 33: Nos. 142-173: Hellenistic painted ware; no. 142: A body fragment of open forms; nos. 143-145: Rim fragments of closed forms; no. 146: A base fragment of closed forms; and nos. 147173: Body fragments of closed forms. 

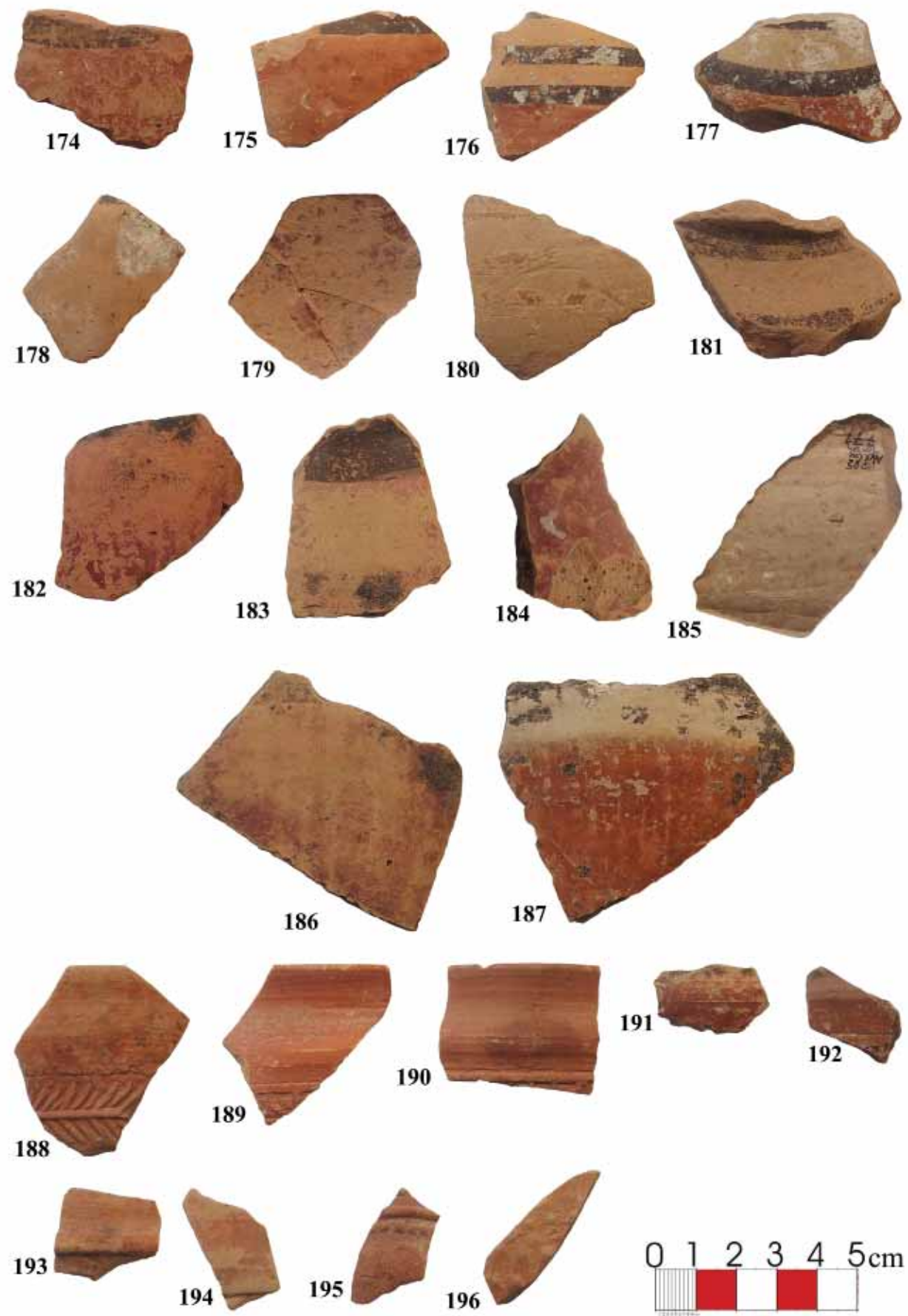

Pl. 34: Nos. 174-187: Hellenistic painted ware, body fragments of closed forms; nos.188196: Hellenistic relief ware; nos. 188-190: Rim fragments of a bowl form; and nos. 191-196: Body fragments of open forms. 


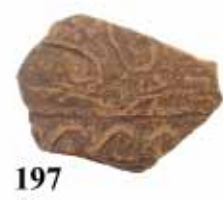

197
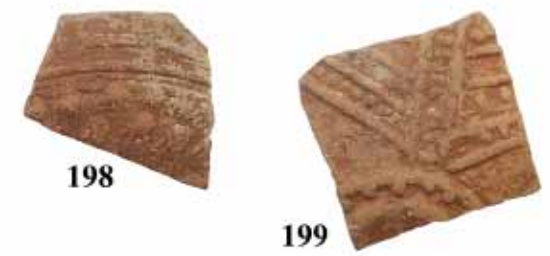

200

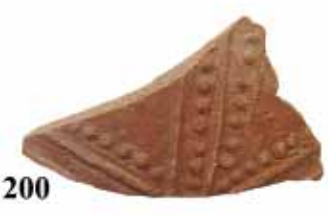

201

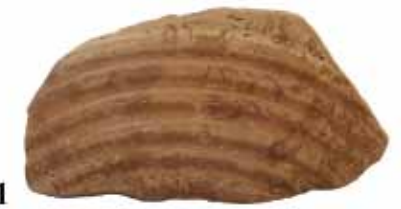

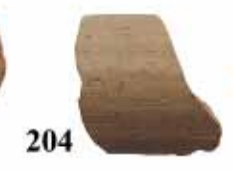
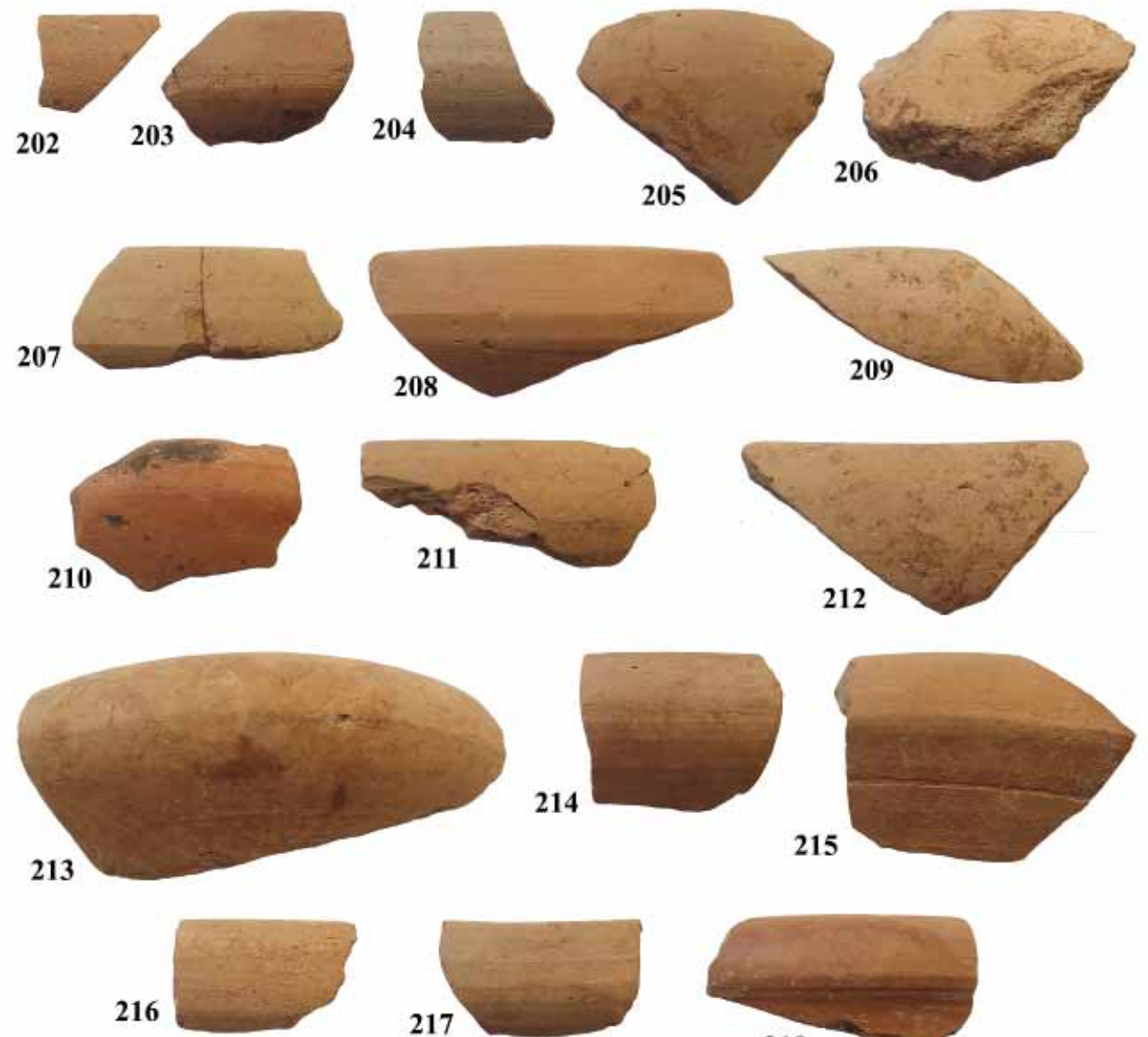
217

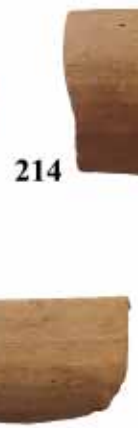

212
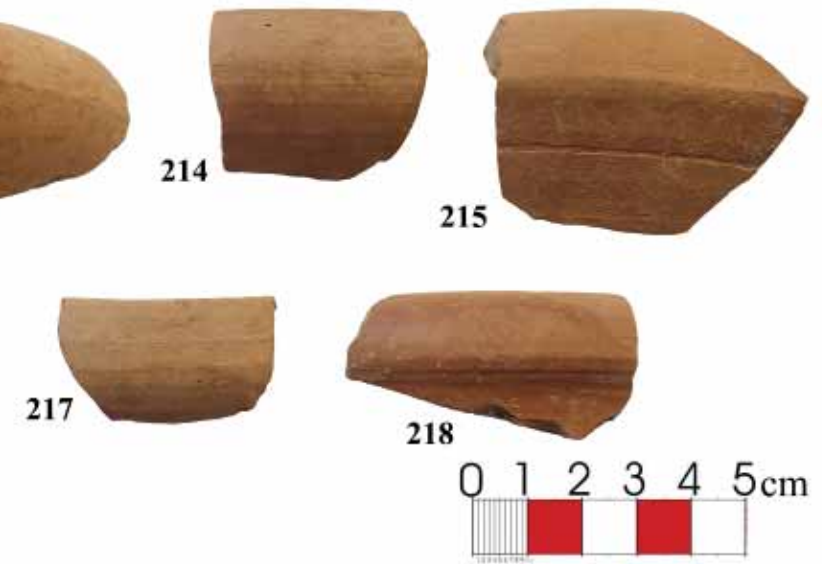

PI. 35: Nos. 197-201: Hellenistic relief ware, body fragments of open forms; and nos. 202-218: Hellenistic burnished ware, rim fragments of a bowl form. 

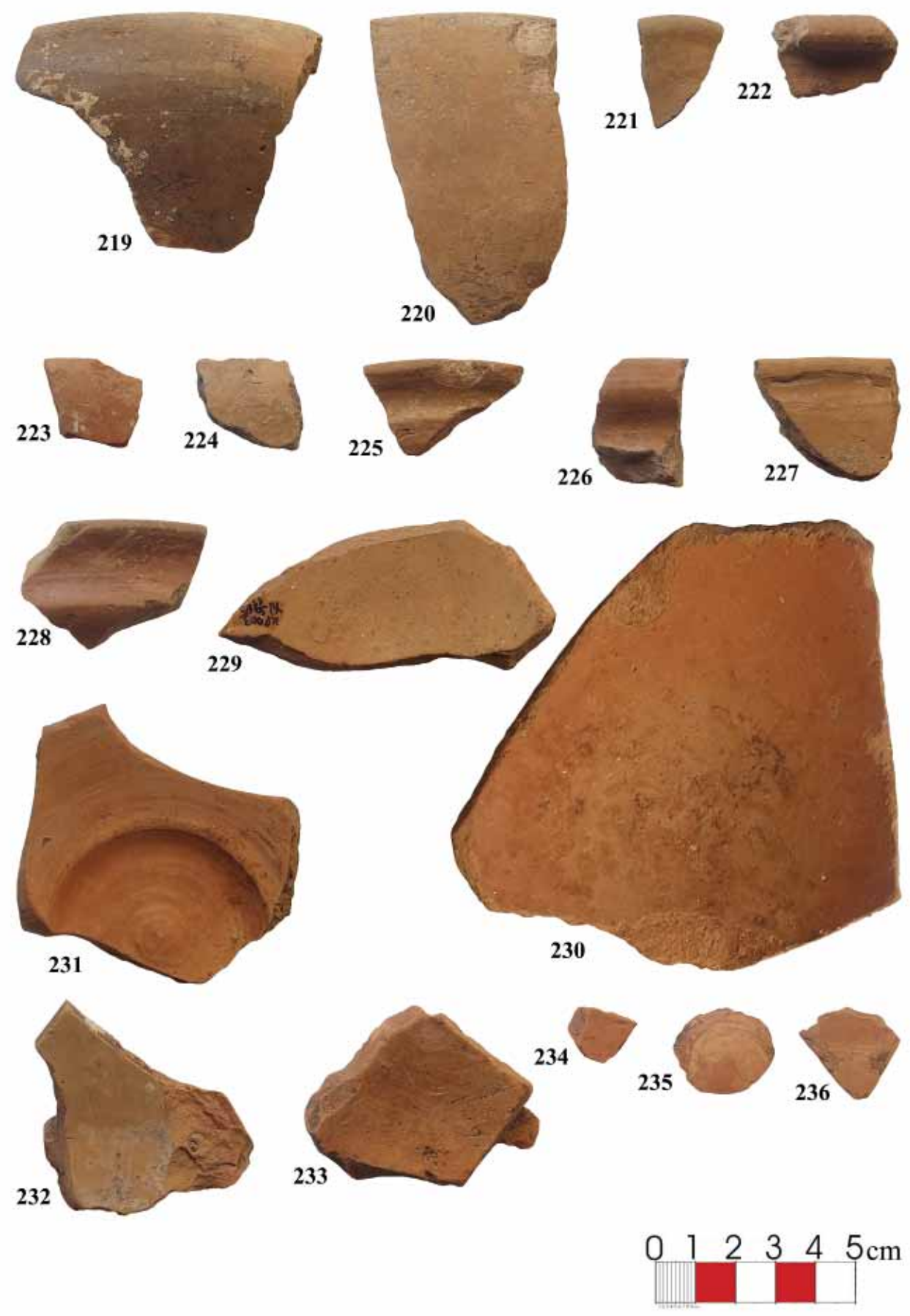

Pl. 36: Nos. 219-236: Hellenistic burnished ware; nos. 219-220: Rim fragments of a bowl form; nos. 221-228: Other rim fragments; nos. 229-233: Base fragments of open forms; and nos. 234236: Body fragments of open forms. 

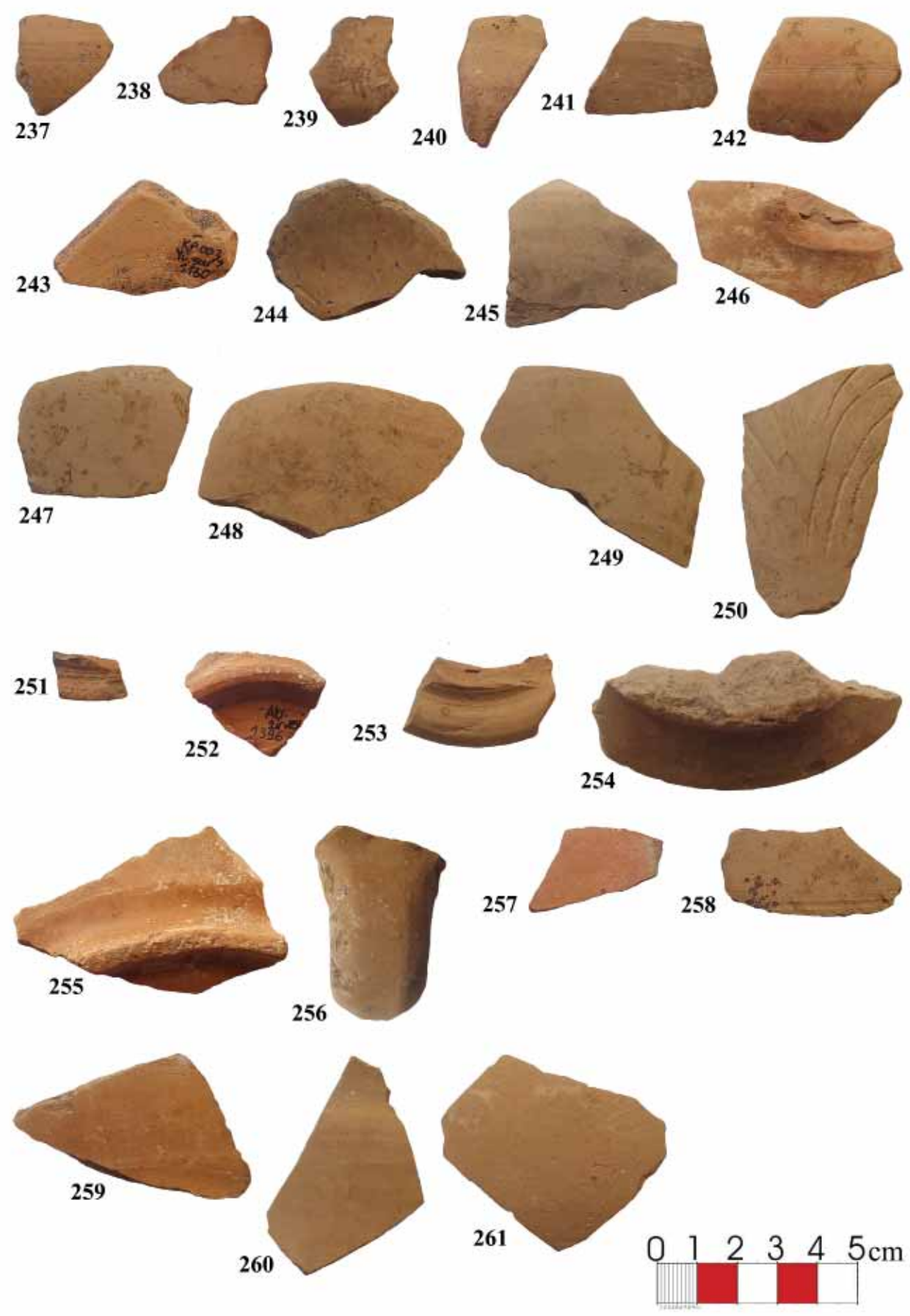

Pl. 37: Nos. 237-261: Hellenistic burnished ware; nos. 237-249: Body fragments of open forms; no. 250: A body fragment of a rhyton; nos. 251-255: Base fragments of closed forms; no. 256:

A handle fragment of closed forms; and nos. 257-261: Body fragments of closed forms. 

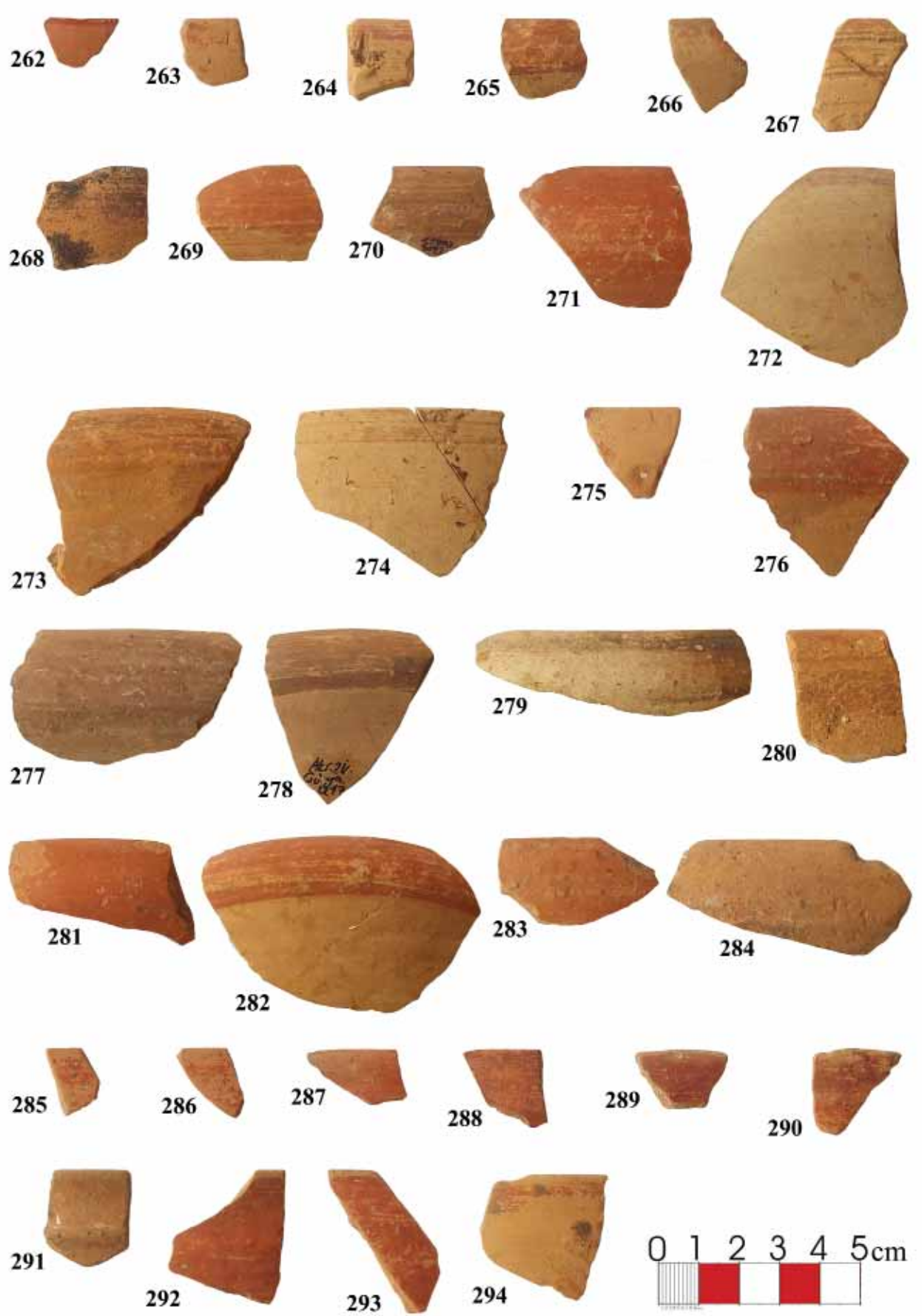

Pl. 38: Nos. 262-294: Red-painted Kepez group; nos. 262-284: Bowl form 1; and nos. 285294: Bowl form 2. 

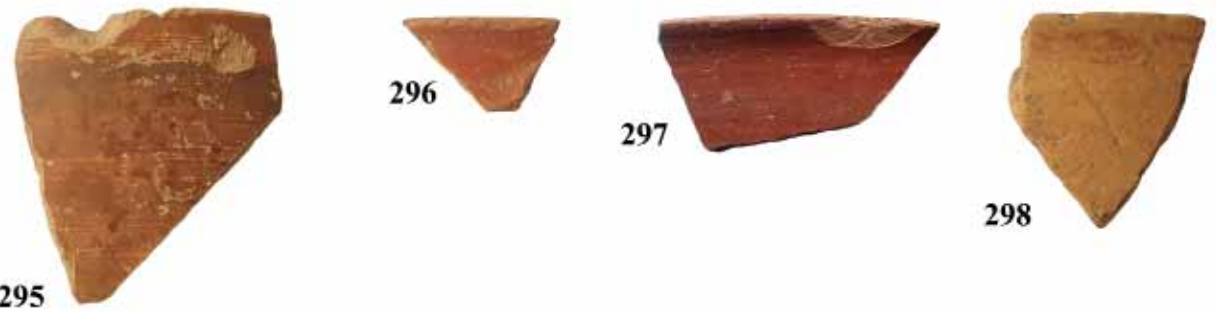

295
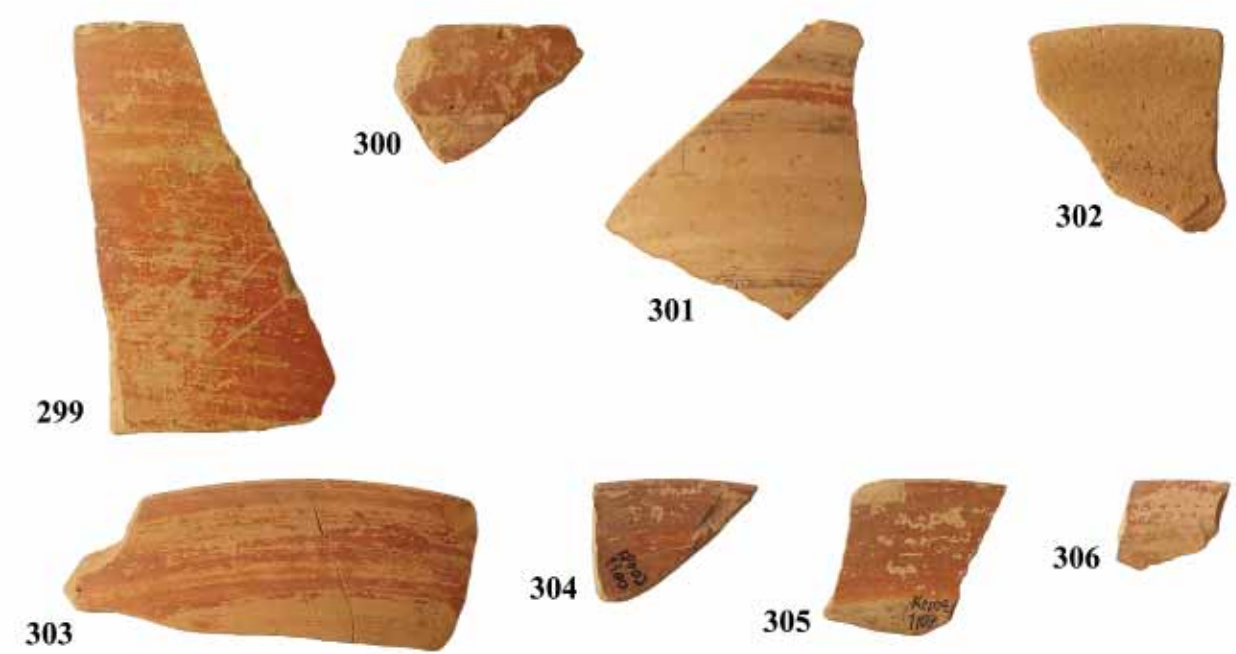

306
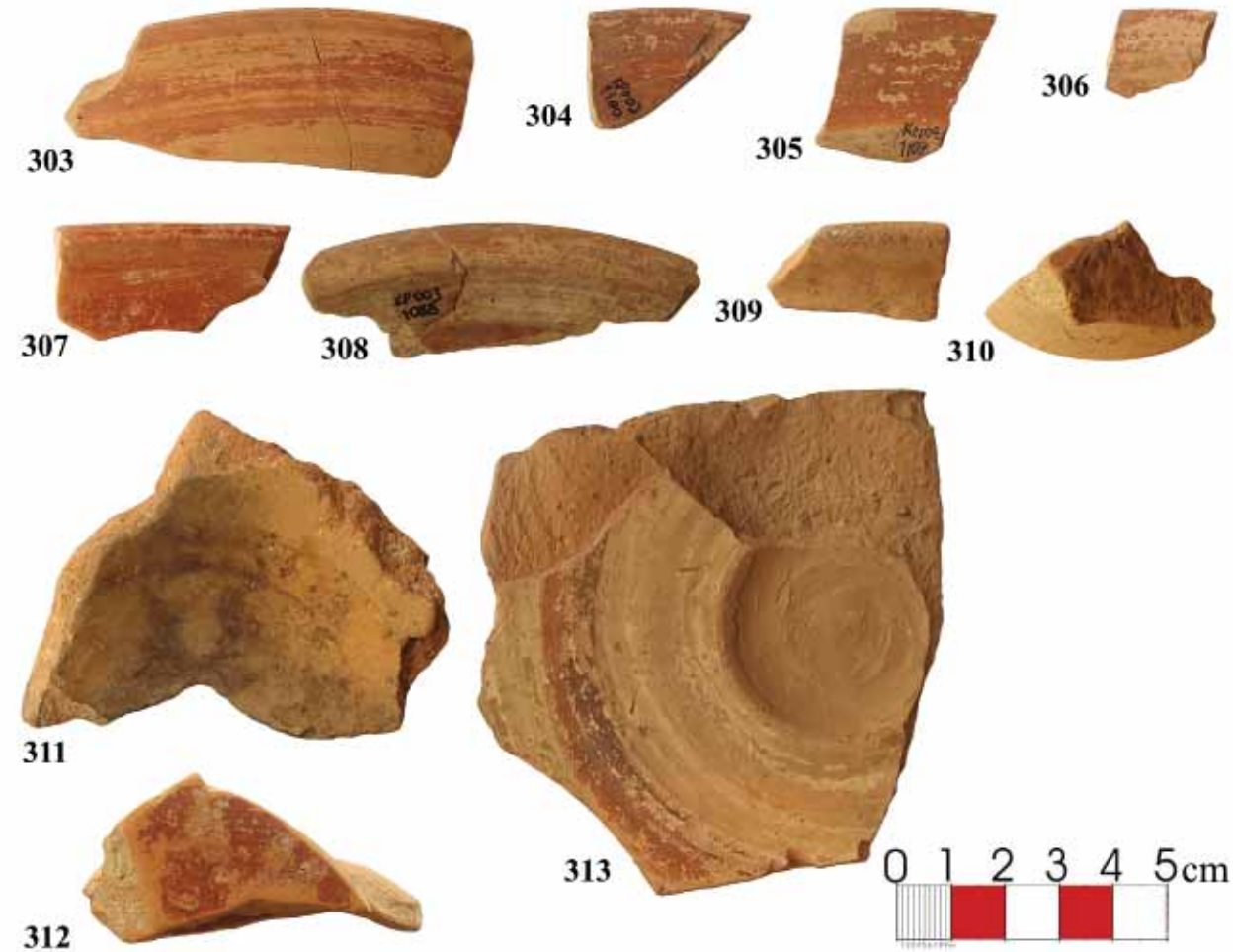

Pl. 39: Nos. 295-313: Red-painted Kepez group; nos. 295-300: Bowl form 2; nos. 301-303: Dish form 1; nos. 304-305: Dish form 2; nos. 306-309: Plate; and nos. 310-313: Base fragments of open forms. 

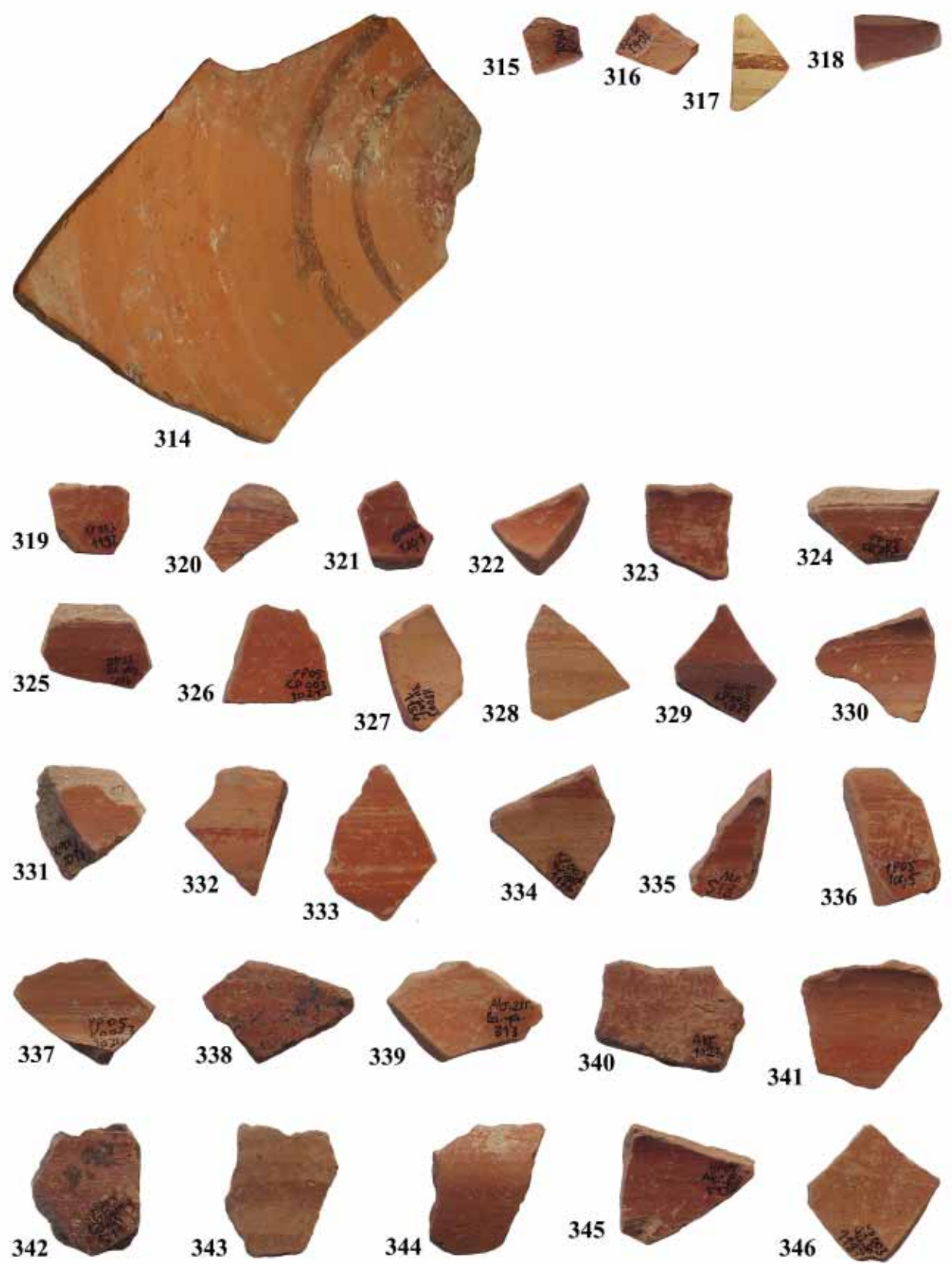

345
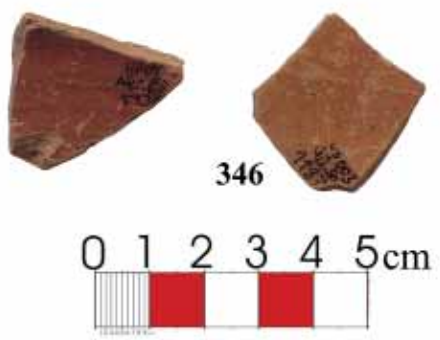

Pl. 40: Nos. 314-346: Red-painted Kepez group; no. 314: A base fragment of open forms; and nos. 315-346: Body fragments of open forms. 

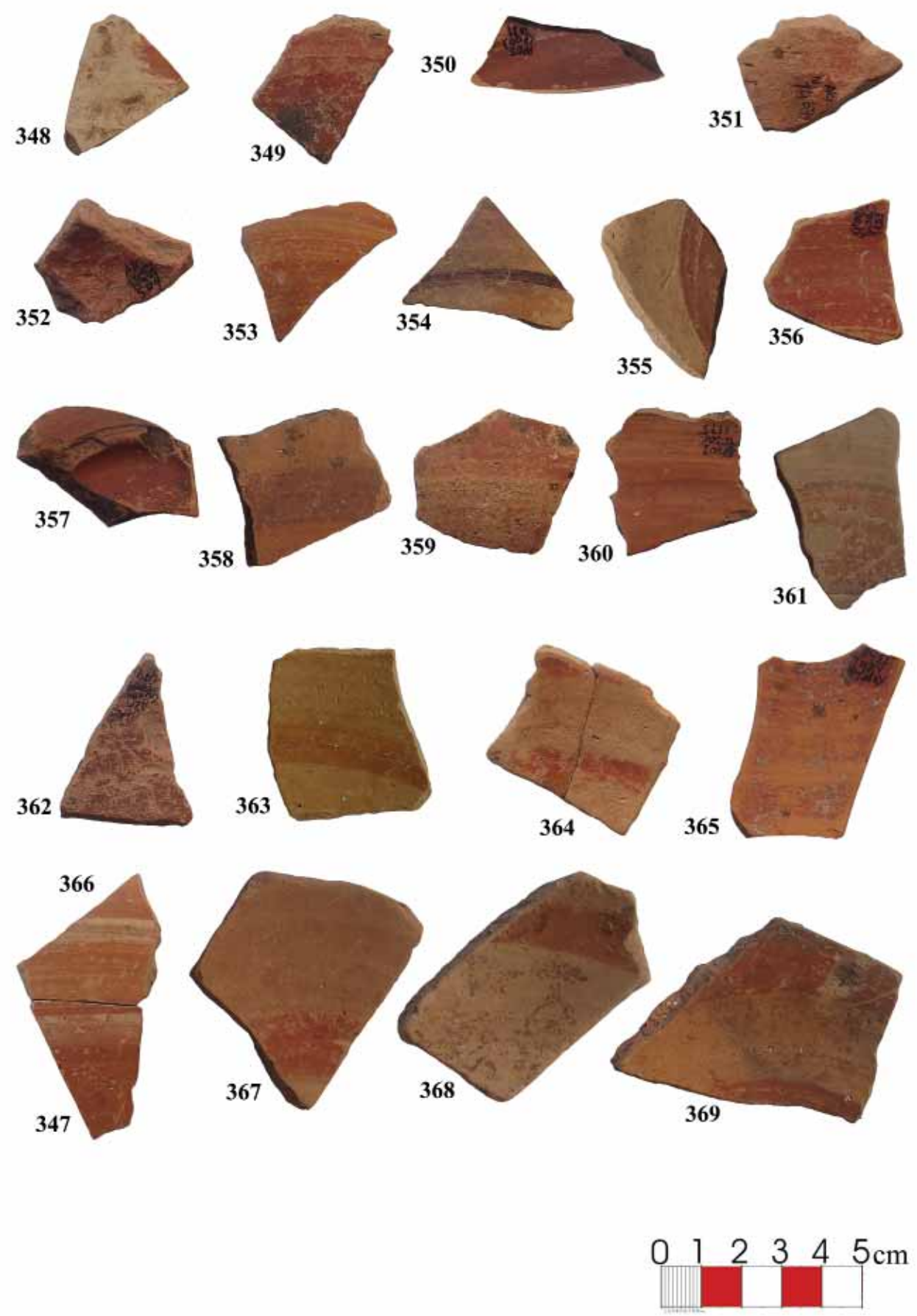

PI. 41: Nos. 347- 369: Red-painted Kepez group, body fragments of open forms. 

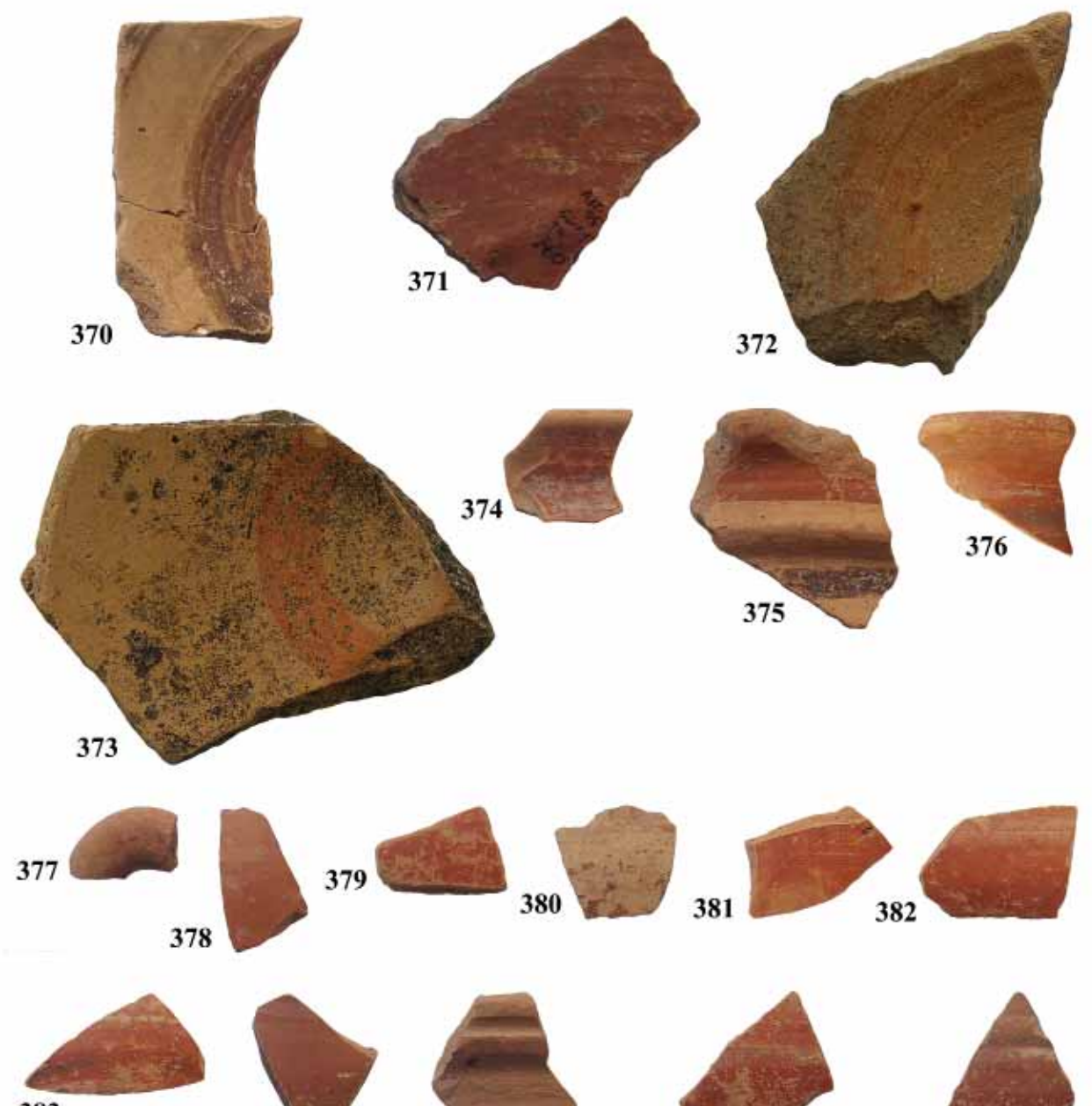

383
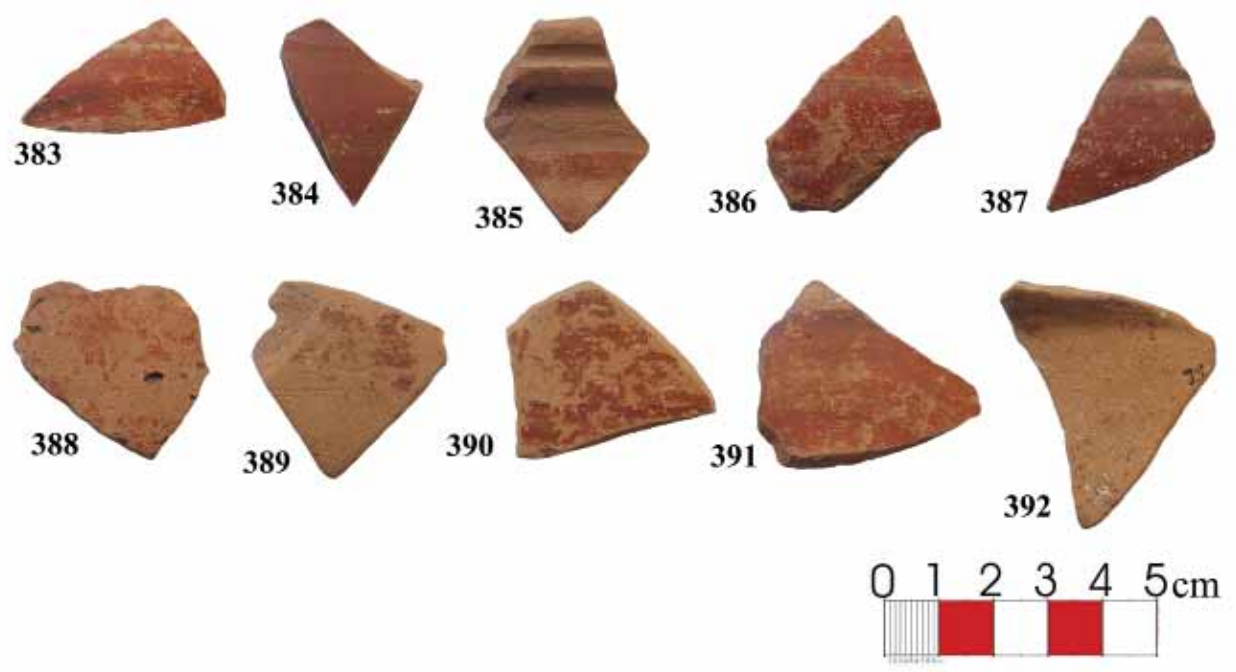

Pl. 42: Nos. 370-392: Red-painted Kepez group; nos. 370-373: Body fragments of open forms; nos. 374-376: Rim fragments of closed forms; no. 377: A handle fragment of closed forms; and nos. 378-392: Body fragments of closed forms. 

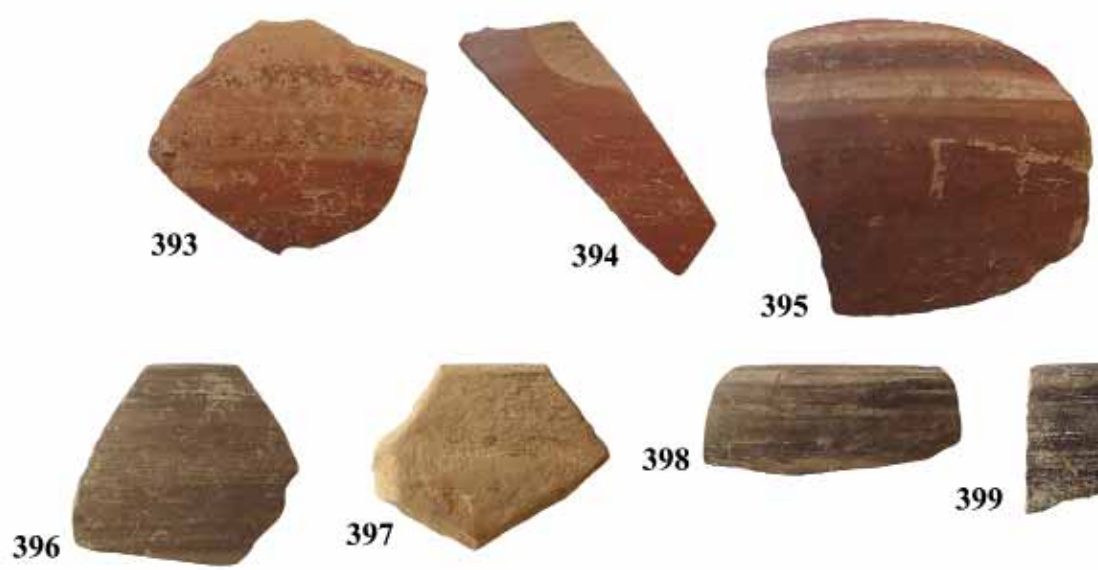

398
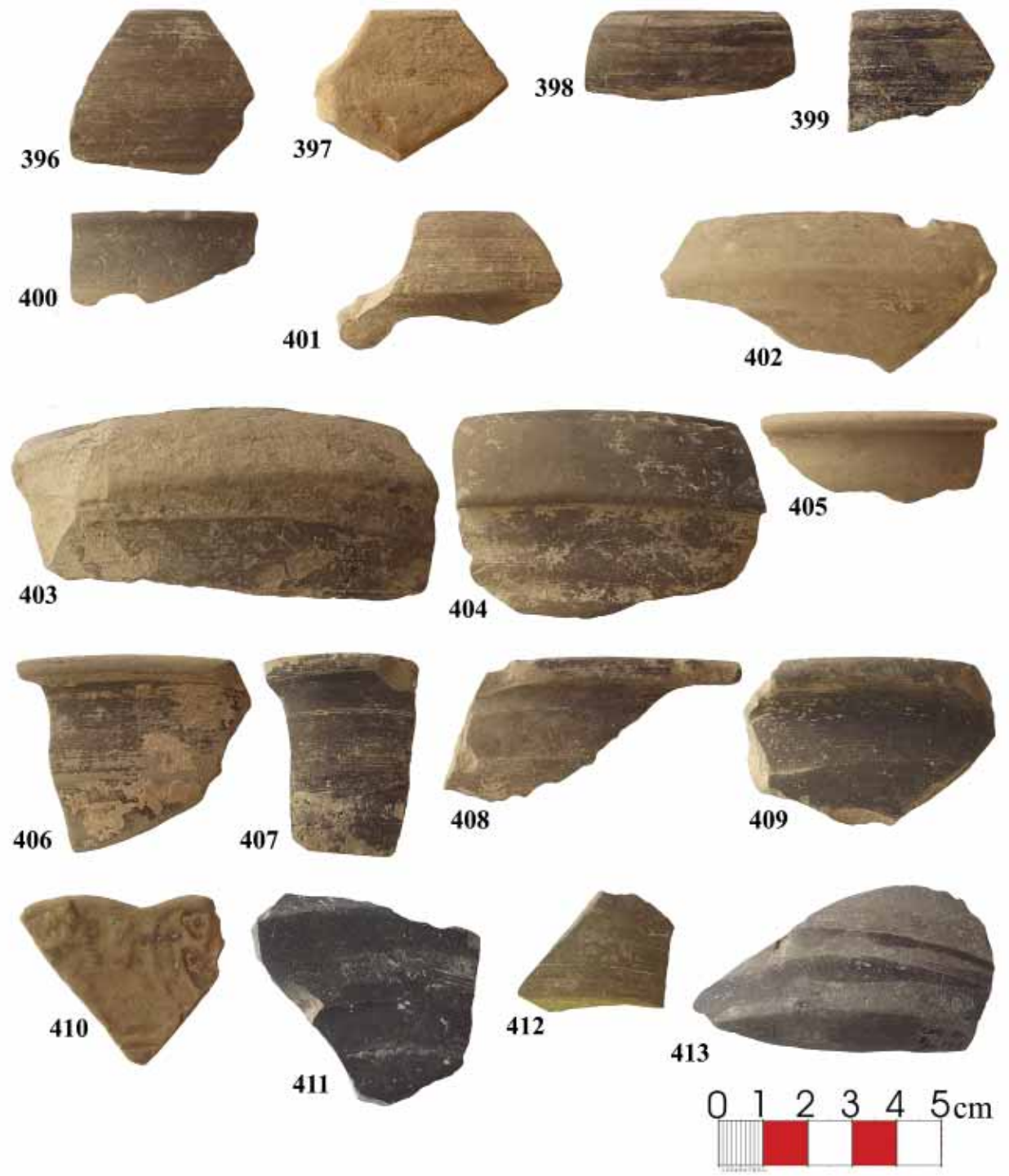

Pl. 43: Nos. 393-395: Red-painted Kepez group; nos. 393-395: Body fragments of closed forms; nos. 396-413: Late Hellenistic-Early Roman grey ware; nos. 396-398: Bowl form 1; nos. 399400: Bowl form 2; nos. 401-404: Dish form 1; nos. 405-409: Dish form 2; no. 410: A rim fragment of a plate; and nos. 411-413: Base fragments of open forms. 

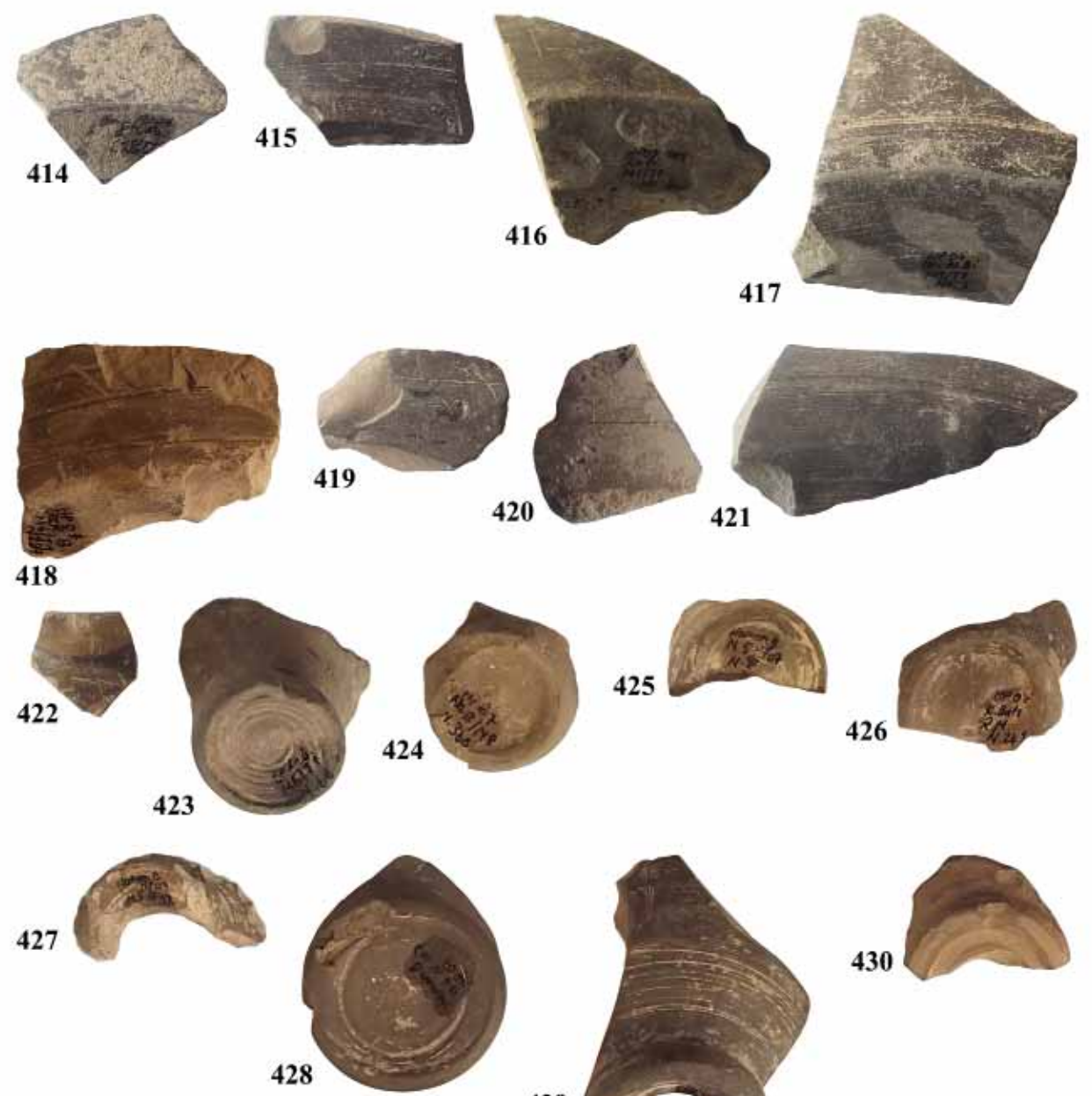

429
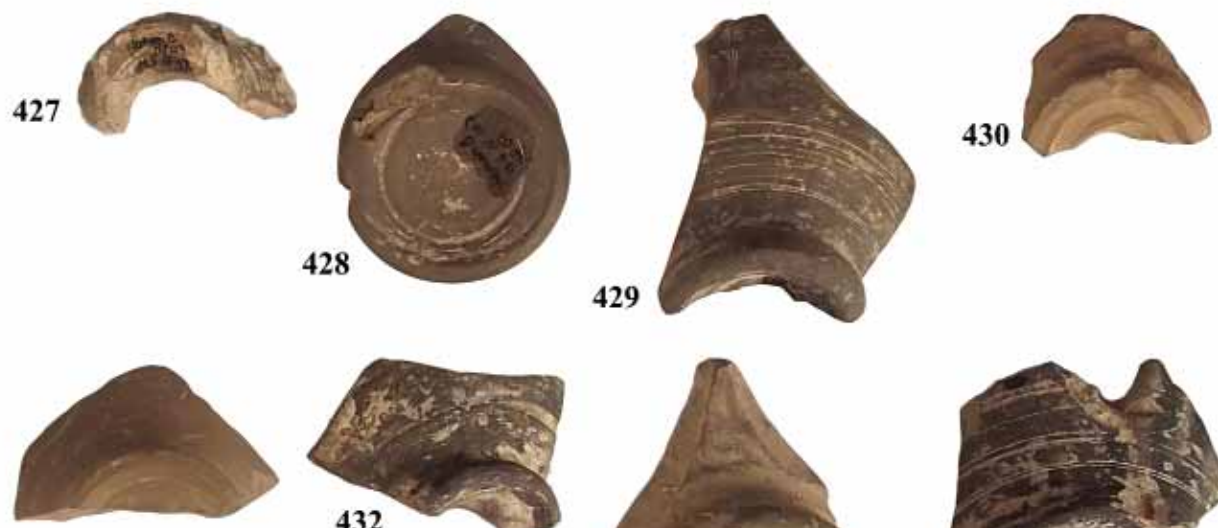

431
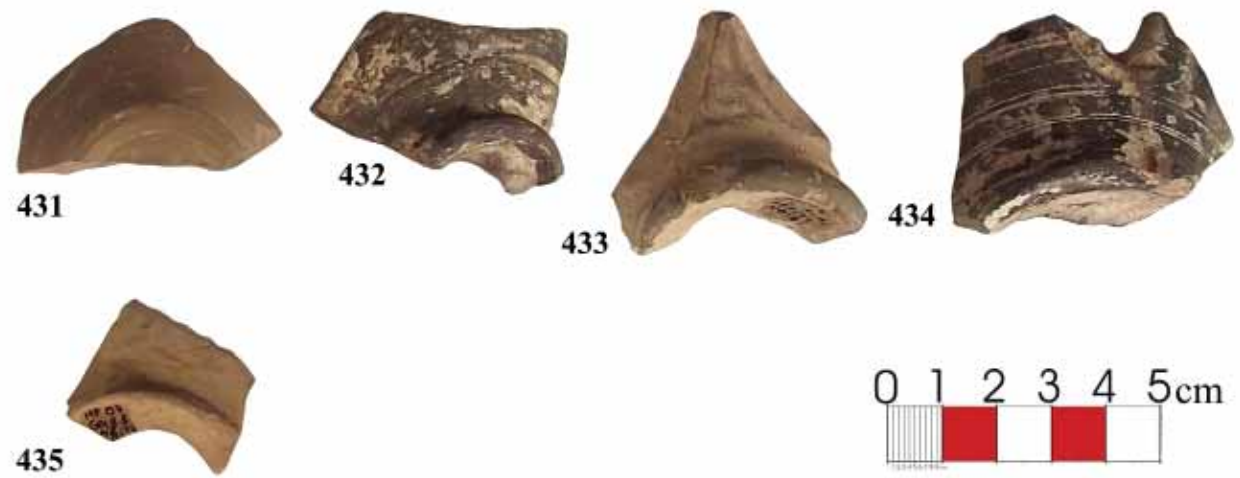

PI. 44: Nos. 414-435: Late Hellenistic-Early Roman grey ware; nos. 414-418: Base fragments of open forms; nos. 419-421: Body fragments of open forms; no. 422: A rim fragment of a juglet; and nos. 423-435: Base fragments of closed forms. 

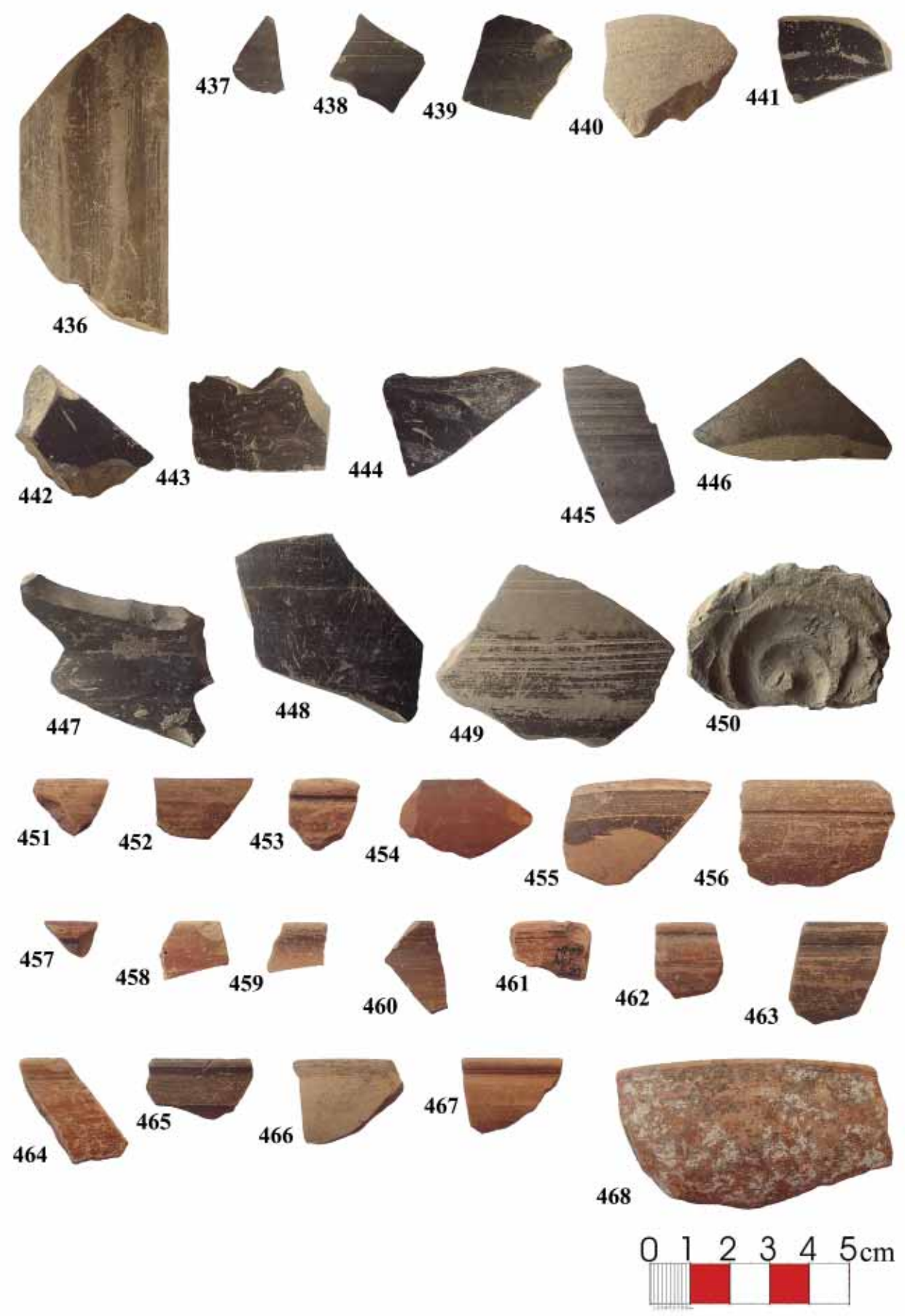

Pl. 45: Nos. 436-450: Late Hellenistic-Early Roman grey ware; no. 436: A handle fragment of closed forms; nos. 437-450: Body fragments of closed forms; nos. 451-468: Late Hellenistic-Early Roman brown-slipped ware; nos. 451-456: Bowl form 1; and nos. 457-468: Bowl form 2. 


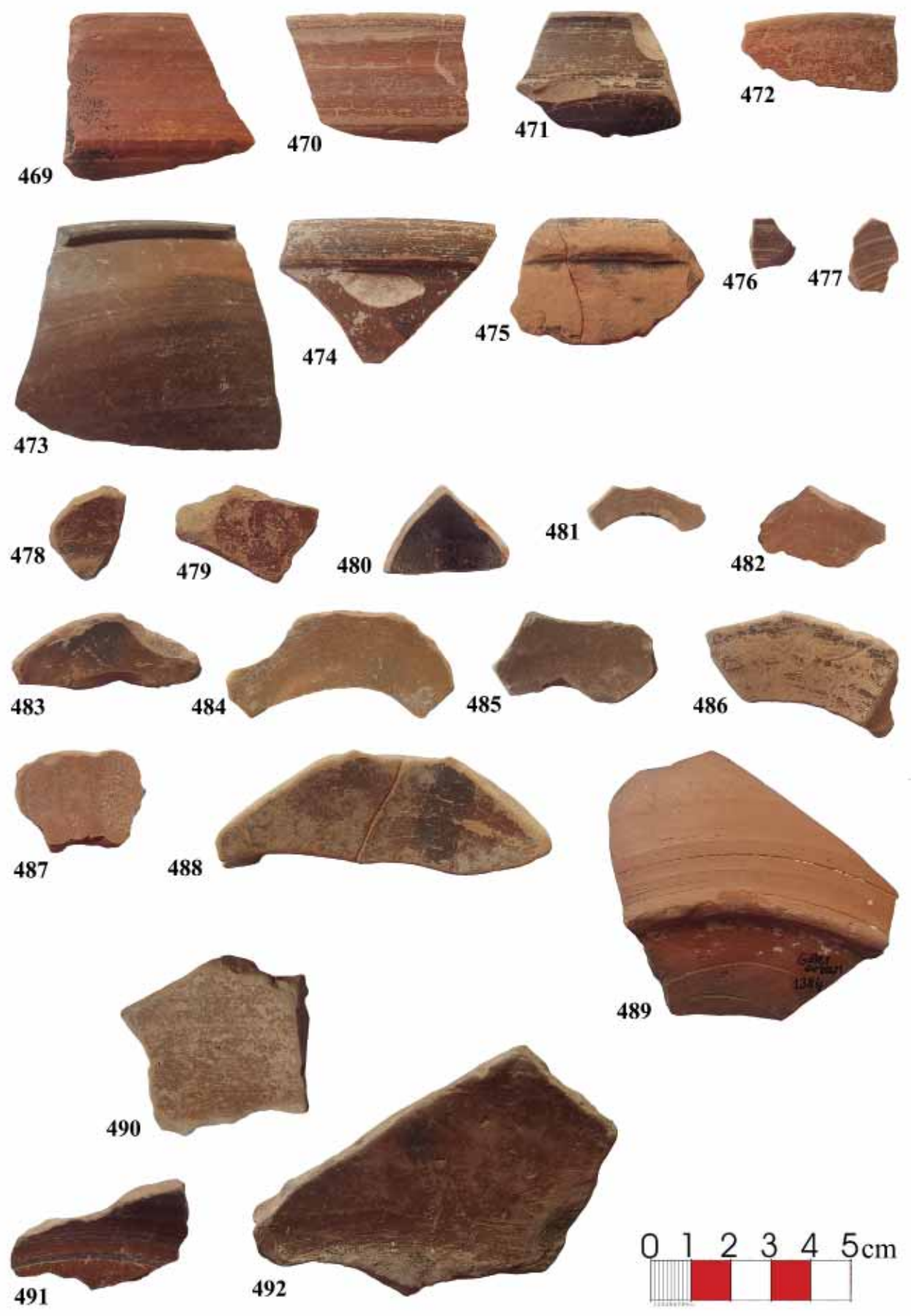

Pl. 46: Nos. 469-492: Late Hellenistic-Early Roman brown-slipped ware; nos. 469-471: Dish; nos. 472-475: Other rim forms; and nos. 476-492: Base fragments of open forms. 

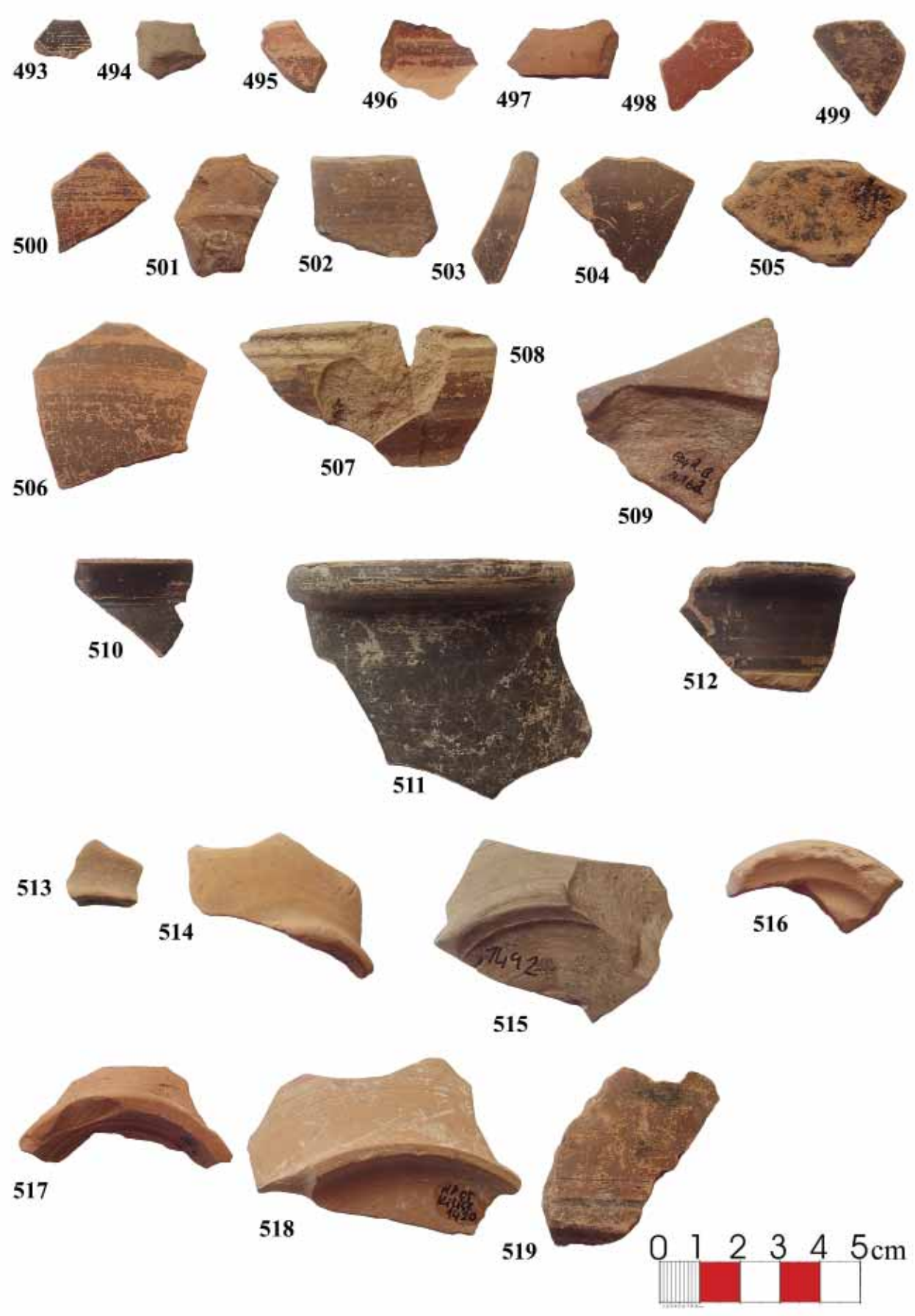

Pl. 47: Nos. 493-519: Late Hellenistic-Early Roman brown-slipped ware; nos. 493-509: Body fragments of open forms; nos. 510-512: Juglet; and nos. 513-519: Base fragments of closed forms. 

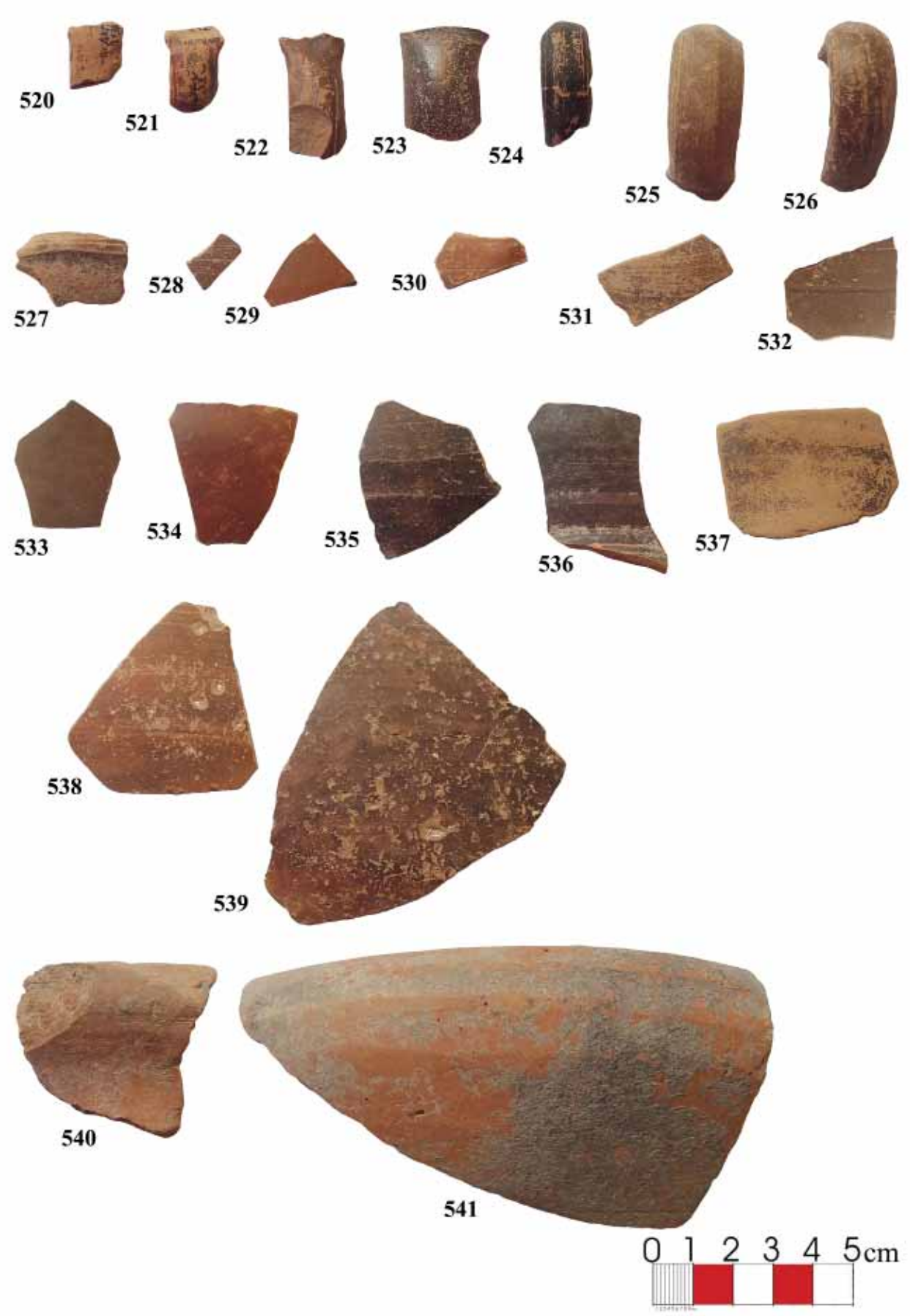

PI. 48: Nos. 520-539: Late Hellenistic-Early Roman brown-slipped ware; nos. 520-526: Handle fragments of closed forms; nos. 527: A lamp fragment; nos. 528-539: Body fragments of closed forms; and nos. 540-541: Hellenistic coarse ware, rim fragments of open forms. 

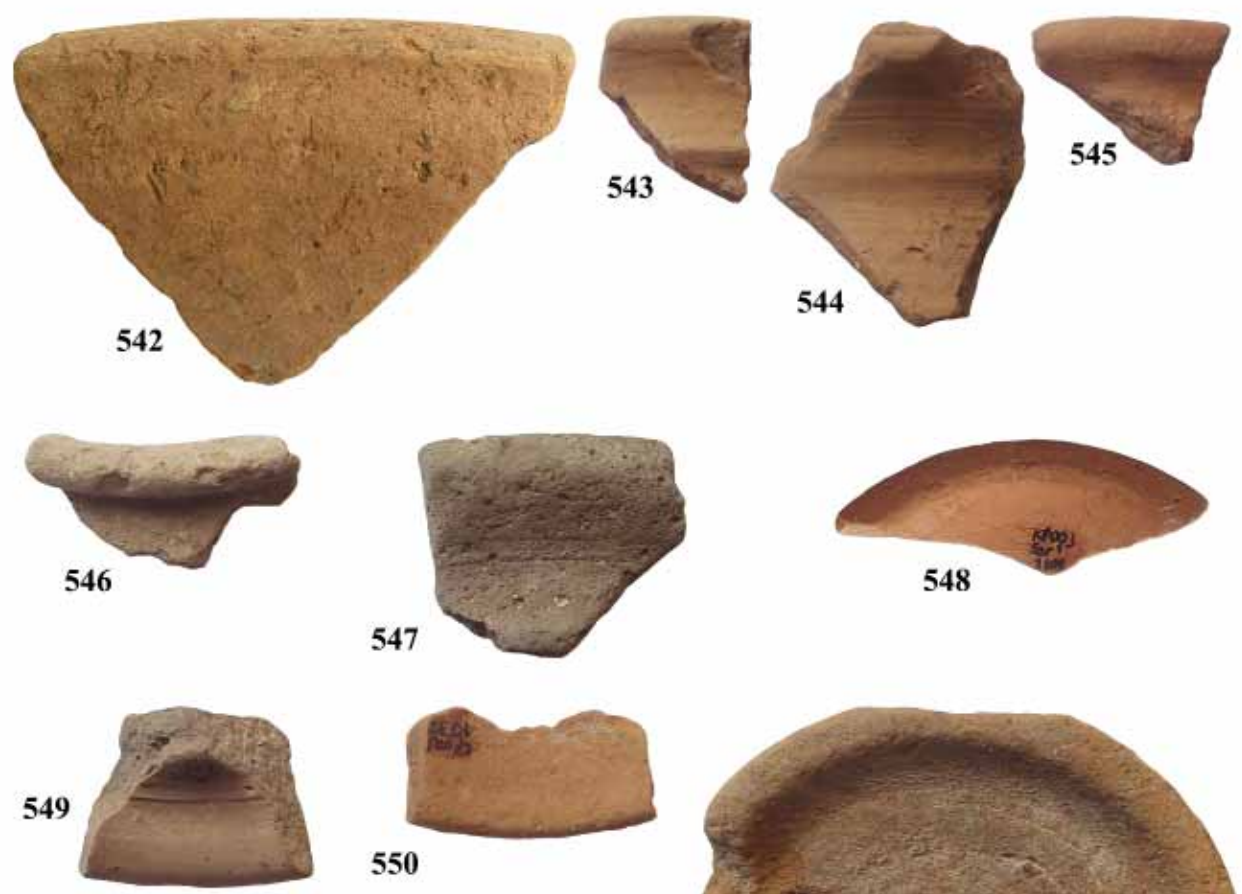

551
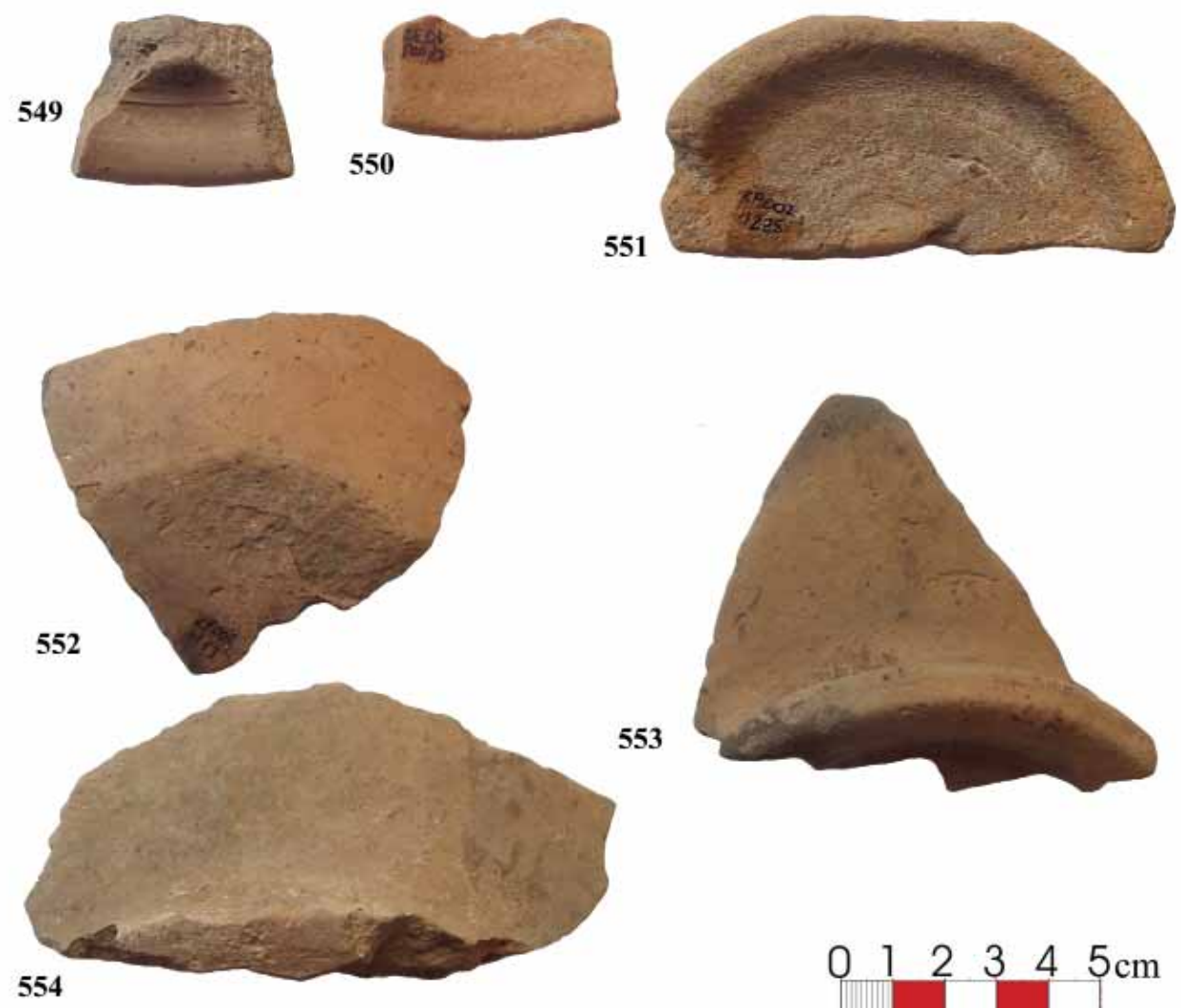

553

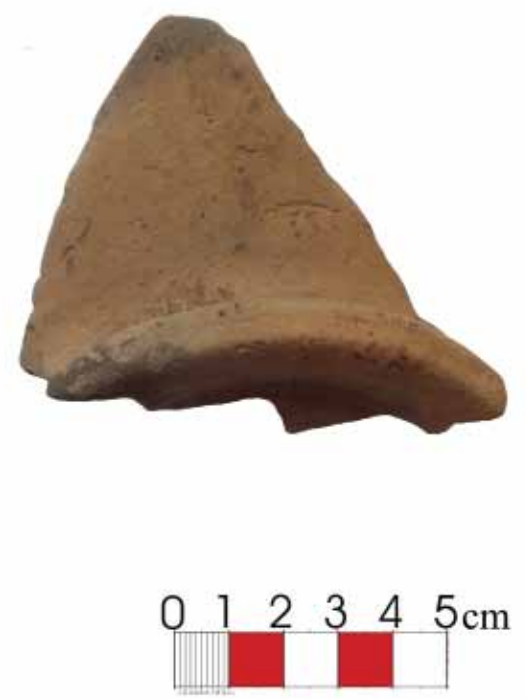

PI. 49: Nos. 542-554: Hellenistic coarse ware; no. 542: A rim fragment of open forms; nos. 543-547: Rim fragments of closed forms; and nos. 548-554: Base fragments of closed forms. 

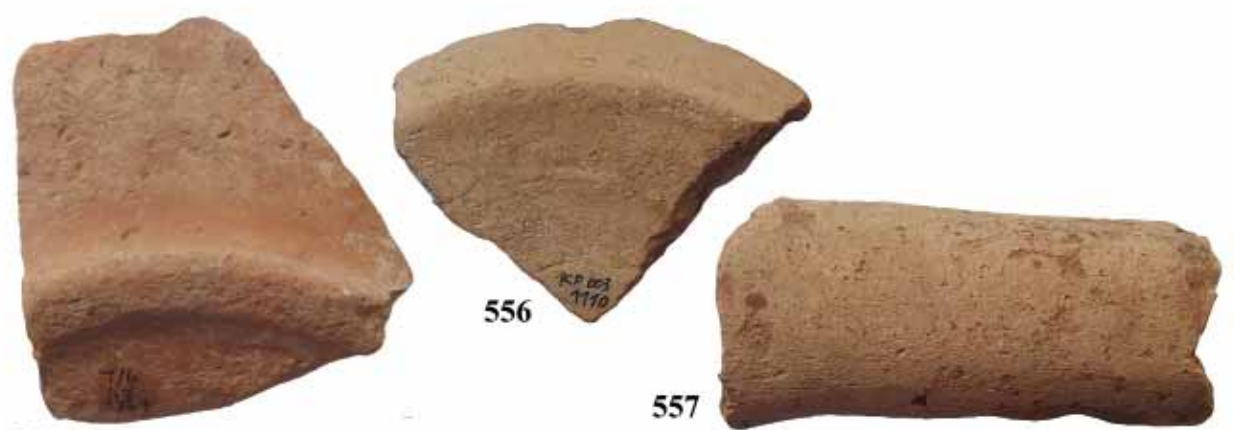

555
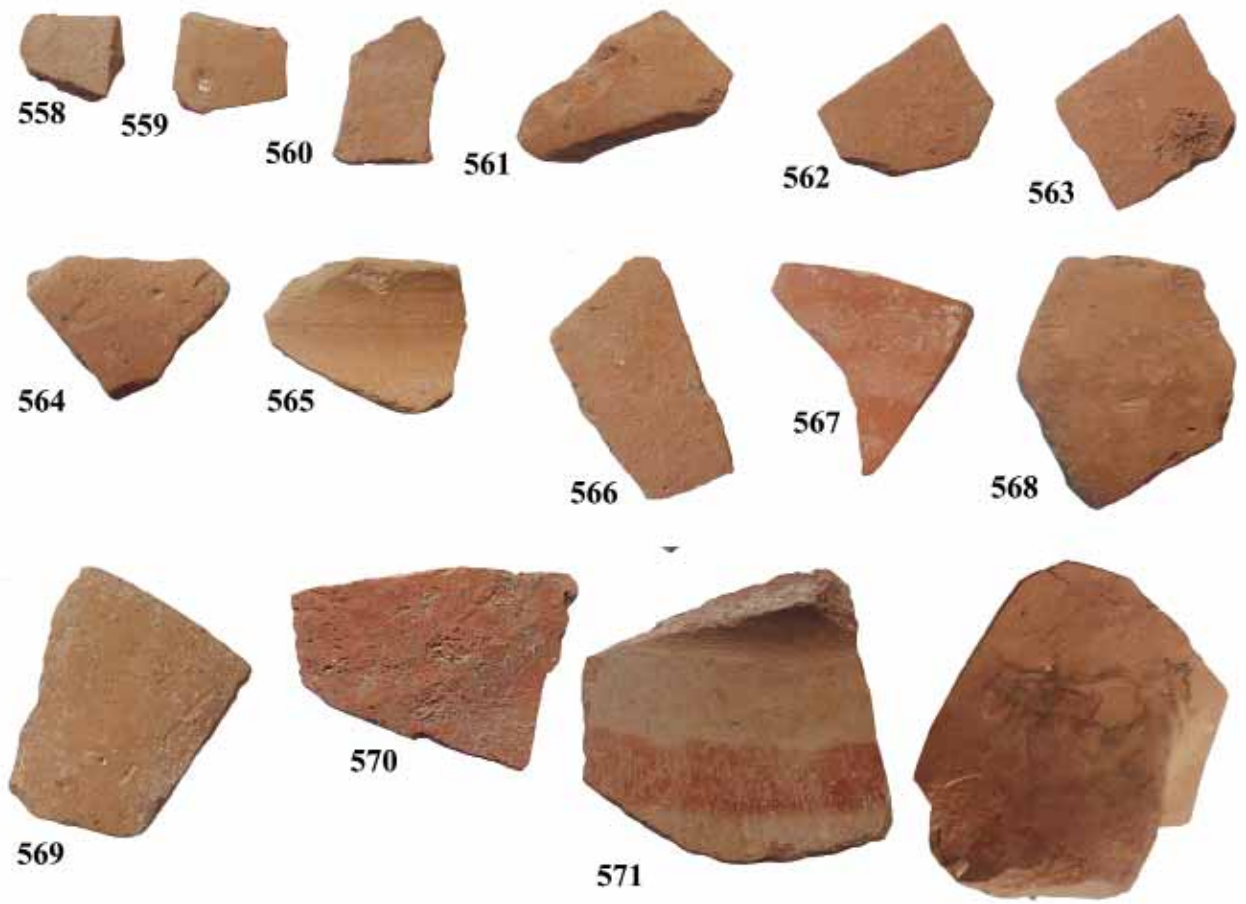

572
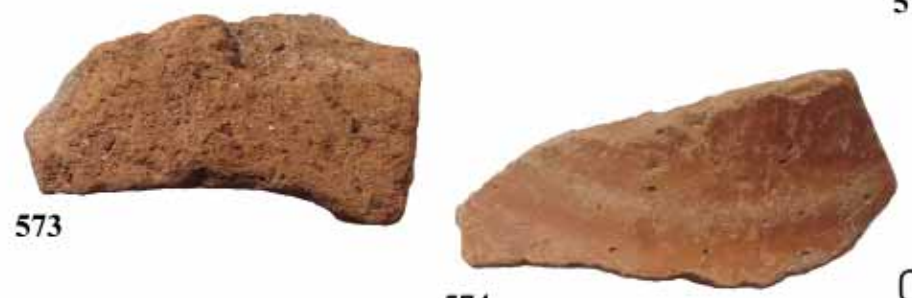

574

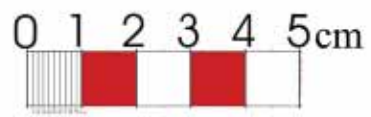

Pl. 50: Nos. 555-574: Hellenistic coarse ware; nos. 555-556: Base fragments of closed forms; no. 557: A handle fragment of closed forms; and nos. 558-574: Body fragments of closed forms. 


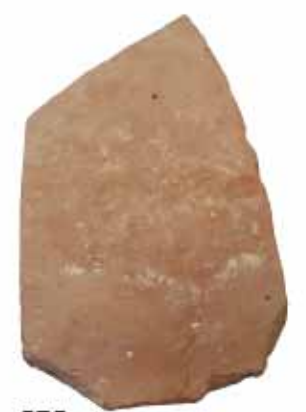

575
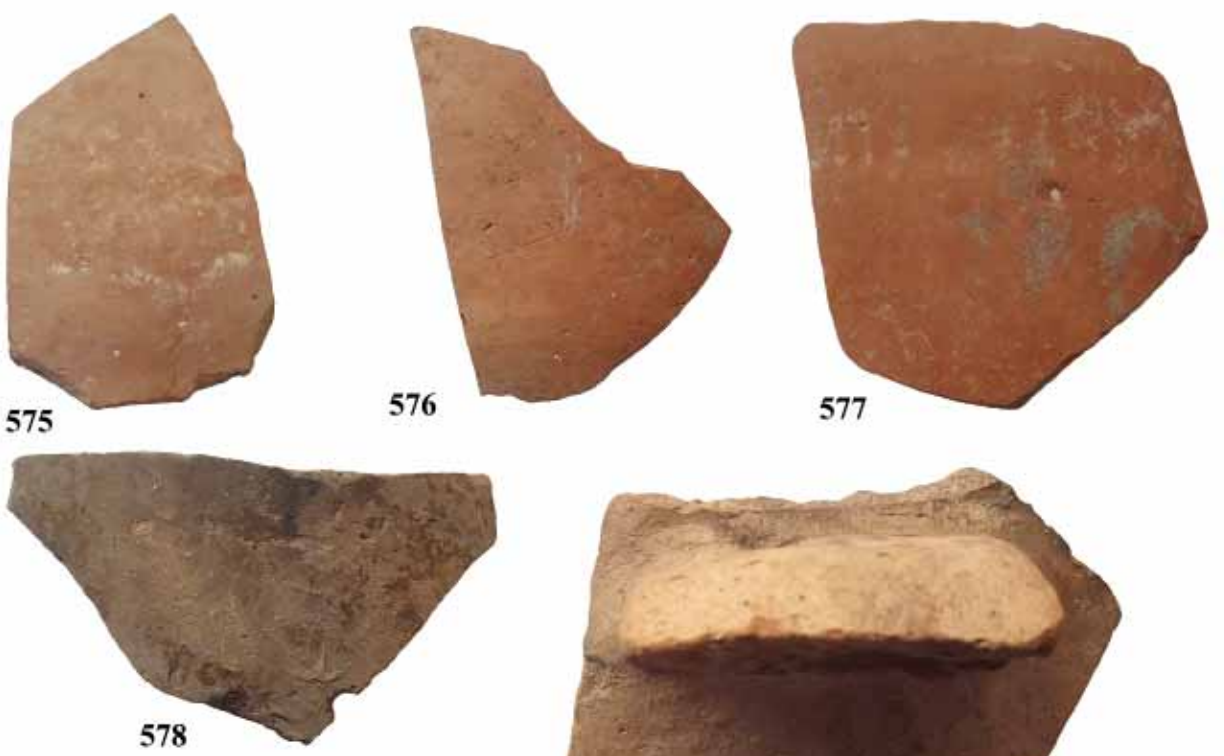

579
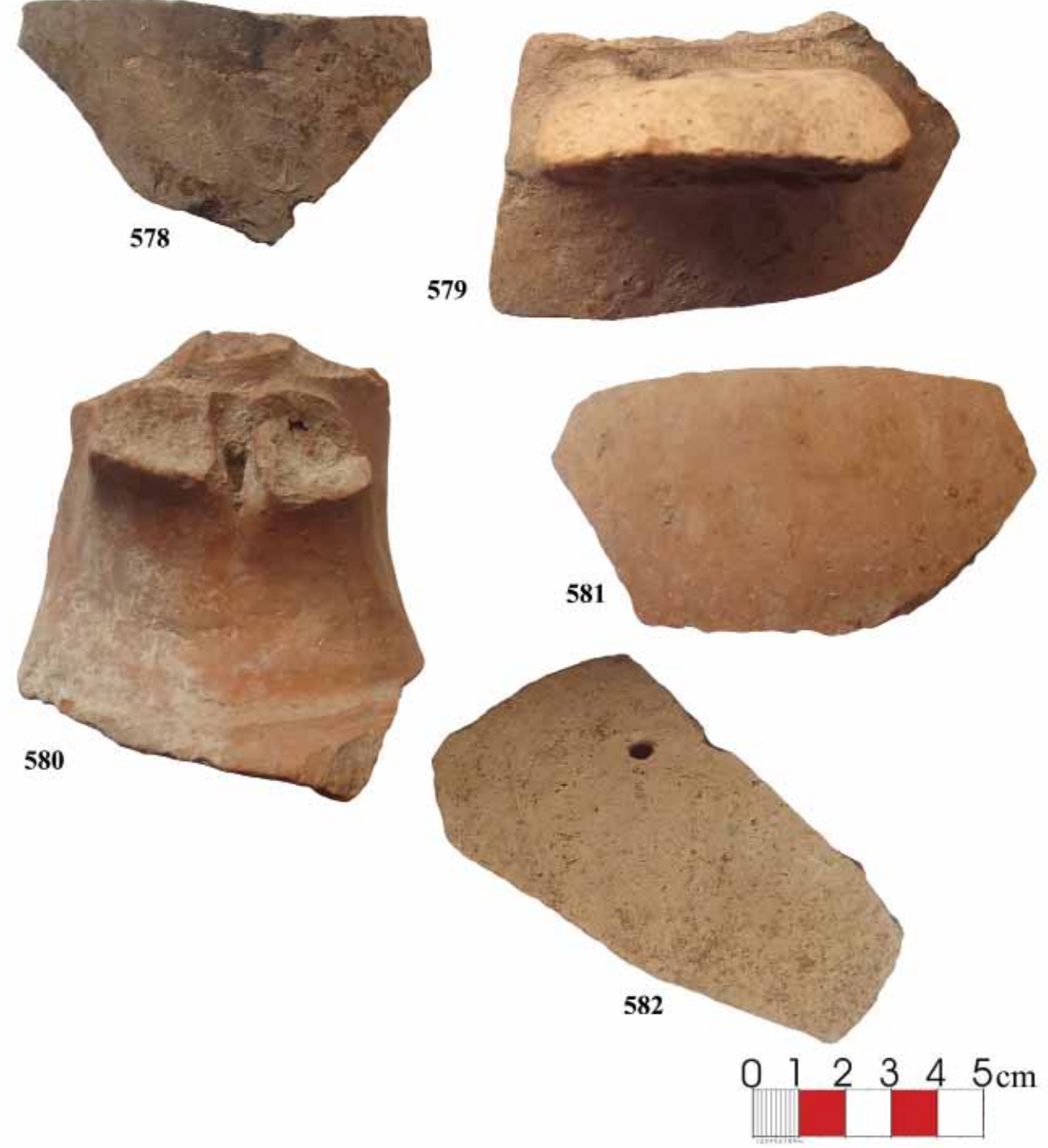

Pl. 51: Nos. 575-582: Hellenistic coarse ware, body fragments of closed forms. 


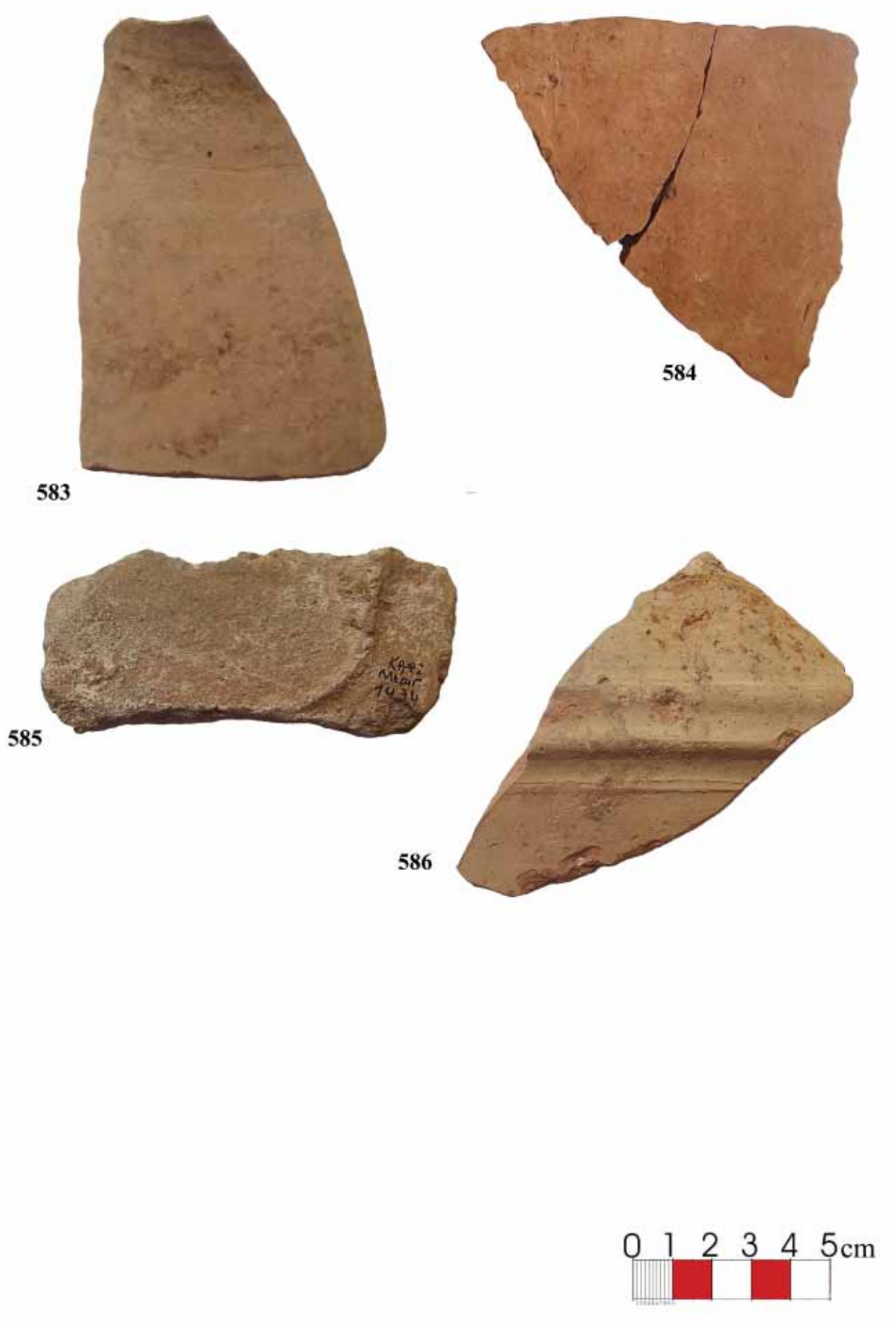

Pl. 52: Nos. 583-586: Hellenistic coarse ware, body fragments of closed forms. 